

\section{OPTIMIZATION OF VORTEX GENERATORS POSITIONS AND ANGLES IN FIN-TUBE COMPACT HEAT EXCHANGER AT LOW REYNOLDS NUMBER}

Thesis Submitted in Partial Fulfillment of the Requirements for the Degree of Doctor of Science in Mechanical

Engineering

Graduate Program of Mechanical Engineering

Supervisor:

Prof. Dr. Jurandir Itizo Yanagihara 
Este exemplar foi revisado e corrigido em relação à versão original, sob responsabilidade única do autor e com a anuência de seu orientador.

São Paulo, 26 de maio de 2014.

Assinatura do autor

Assinatura do orientador

Salviano, Leandro Oliveira

Optimization of vortex generators positions

and angles in fin-tube compact heat exchanger at Low Reynolds Number / L.O. Salviano. - versão corr. -- São Paulo, 2014.

$218 p$.

Tese (Doutorado) - Escola Politécnica da Universidade de São Paulo. Departamento de Engenharia Mecânica.

1.Transferência de calor (Otimização) 2.Trocadores de calor 3.Algoritmos genéticos 4.Redes neurais 5.Gerador de vórtices I.Universidade de São Paulo. Escola Politécnica. Departamento de Engenharia Mecânica II.t. 


\section{EPIGRAPH}

"For my thoughts are not your thoughts, neither are your ways my ways, declares the LORD. As the heavens are higher than the earth, so are my ways higher than your ways and my thoughts than your thoughts."

lsaiah 55, 8-9 


\section{RESUMO}

Por muitos anos, a intensificação da transferência de calor tem despontado como um importante tópico de pesquisa. Embora existam muitas técnicas eficazes de intensificação da transferência de calor, como o uso de geradores de vórtices, poucos trabalhos de pesquisa lidam com a otimização.

Neste trabalho, foi realizada a otimização das posições e ângulos dos geradores de vórtice longitudinal (LVG) tipo meia asa delta, considerando um trocador de calor tubo-aleta compacto com duas linhas de tubos desalinhados. Duas abordagens foram empregadas: Método da Superfície de Resposta (Neural Networking) e Otimização Direta. Um software comercial (Fluent), baseado na metodologia de volumes finitos, foi empregado na análise numérica da transferência de calor, estruturas vorticais e perda de pressão no escoamento, na presença de LVG. A razão de aspecto dos geradores de vórtice foi 2 e o número de Reynolds, baseado na distâncias entre as aletas, foram de 250 e 1400.

Foram analisados quatro parâmetros dos LVG, os quais impactam na performance do trocador de calor: a posição do LVG na direção $x-y$, o ângulo de ataque $(\theta)$ e o ângulo de rolamento $(\varphi)$. $O$ ângulo de rolamento foi primeiramente estudado neste trabalho. No total, oito parâmetros independentes do LVG foram considerados: $\left(\mathrm{x}_{1}, \mathrm{y}_{1}, \theta_{1}, \varphi_{1}\right)$ para o primeiro tubo e $\left(\mathrm{x}_{2}, \mathrm{y}_{2}, \theta_{2}, \varphi_{2}\right)$ para o segundo tubo. O método da Análise Fatorial (software ModeFrontier) foi aplicado no estudo da influência destes parâmetros dos LVG na performance do trocador de calor. Também foi avaliado o efeito de cada um destes parâmetros na transferência de calor e perda de pressão do escoamento, expressos em termos do fator de Colburn (j) e do fator de Atrito (f), respectivamente.

As configurações otimizadas dos LVG, conduziram à taxas de transferência de calor maiores do que aquelas reportadas pela literatura. A Otimização Direta mostrou resultados melhores do que através da metodologia de Superfície de Resposta para todas as funções objetivas avaliadas neste trabalho. Importantes interações foram identificadas entre $V G_{1}$ e $V G_{2}$, os quais influenciaram nos resultados dos fatores de Colburn (j) e Atrito (f) para cada número de Reynolds. Particularmente, foi identificado que a assimetria dos LVG desempenha um papel fundamental na 
intensificação da transferência de calor, onde os parâmetros de $V G_{2}$ depende fortemente dos parâmetros de $V G_{1}$. Além disso, para cada número de Reynolds e para cada função objetivo, existe uma configuração ótima dos parâmetros do LVG. Se o objetivo é a redução da perda de pressão global, $V_{G_{1}}$ poderia ser suprimido da modelagem, pois a sua principal função é aumentar a transferência de calor ao longo da aleta. Por outro lado, $\mathrm{VG}_{2}$ foi relevante tanto para aumentar a transferência de calor quanto para diminuir a perda de pressão. O ângulo de rolamento teve grande influência sobre o resultado do fator de Atrito (f), especialmente para $V_{G_{1}} \mathrm{e}$ para baixo número de Reynolds.

Palavras-chave: Intensificação da Transferência de Calor, Otimização, Trocador de Calor, Gerador de Vórtice, Algoritmo Genético, Rede Neural. 


\section{ABSTRACT}

In the last few decades, augmentation of heat transfer has emerged as an important research topic. Although many promising heat transfer enhancement techniques have been proposed, such as the use of longitudinal vortex generators, few researches deal with thermal optimization.

In the present work, it was conducted an optimization of delta winglet vortex generators position and angles in a fin-tube compact heat exchanger with two rows of tubes in staggered tube arrangement. Two approaches were evaluated: Response Surface Methodology (Neural Networking) and Direct Optimization. Finite-Volume based commercial software (Fluent) was used to analyze heat transfer, flow structure and pressure loss in the presence of longitudinal vortex generators (LVG). The delta winglet aspect ratio was 2 and the Reynolds numbers, based on fin pitch, were 250 and 1400.

Four vortex generator parameters which impact heat exchanger performance were analyzed: LVG position in direction $x-y$, attack angle $(\theta)$ and roll angle $(\varphi)$. The present work is the first to study the influence of LVG roll angle on heat transfer enhancement. In total, eight independent LVG parameters were considered: $\left(\mathrm{x}_{1}, \mathrm{y}_{1}, \theta_{1}, \varphi_{1}\right)$ for the first tube and $\left(\mathrm{x}_{2}, \mathrm{y}_{2}, \theta_{2}, \varphi_{2}\right)$ for the second tube. Factor Analysis method (software ModeFrontier) was used to study of the influence of these LVG parameters in heat exchanger performance. The effect of each LVG parameter on heat transfer and pressure loss, expressed in terms of Colburn factor (j) and Friction factor (f), respectively, were evaluated.

The optimized LVG configurations led to heat transfer enhancement rates that are much higher than reported in the literature. Direct Optimization reported better results than Response Surface Methodology for all objective functions. Important interactions were found between $V G_{1}$ and $V G_{2}$, which influenced the results of Colburn (j) and Friction (f) factors for each Reynolds number. Particularly, it was found that the asymmetry of the LVG, in which the $V^{2}$ parameters strongly depend on the $V G_{1}$ parameters, plays a key role to enhance heat transfer. Moreover, for each Reynolds number and each objective function, there is an optimal LVG arrangement. 
If the objective is to mitigate pressure drop, $V G_{1}$ may be suppressed because its main goal is increasing the heat transfer downstream. On the other hand, $V G_{2}$ was relevant for both increase the heat transfer and decrease the pressure drop. Roll angle had a strong influence on Friction factor ( $f$ ), especially for $V G_{1}$ and low Reynolds number.

Keywords: Heat Transfer Enhancement, Optimization, Heat Exchanger, Vortex Generator, Genetic Algorithm, Neural Network. 


\section{LIST OF ILLUSTRATIONS}

Figure 1 - Fin-tube CHE. (a) Mechanical Heat Exchanger (MHE), (b) Brazed Heat Exchanger (BHE). 3 Figure 2 - Example of twisted tapes, (a) single swirl flow, (b) co-swirl flow and (c) counter-swirl flow, Eiamsa-Ard et al. (2010).

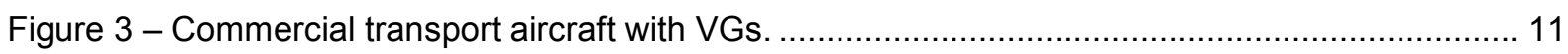

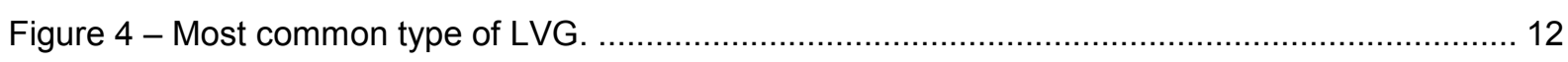

Figure 5 - Transverse vortex behind ribs in a channel (FIEBIG, 1998)......................................... 13

Figure 6 - Sketch of vortical structures generated by delta-winglet (YANAGIHARA AND TORII, 1991).

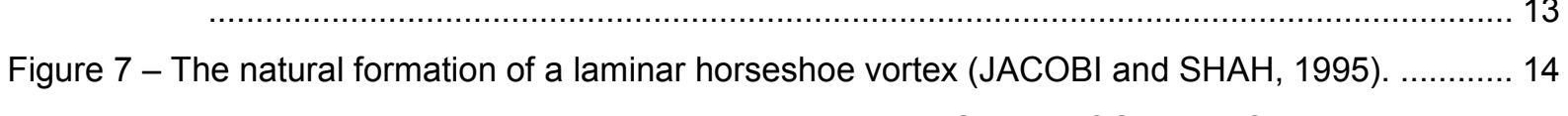

Figure 8 - Typical passively generated longitudinal vortices in CHE (JACOBI and SHAH, 1995)...... 14

Figure 9 - Schematic diagram of core region of a slit fin-and-tube heat exchanger with longitudinal vortex generators (Li et al., 2011) .............................................................................. 15

Figure 10 - (a) Configuration of the VG geometric proposed, (b) Schematic of the flow motions across

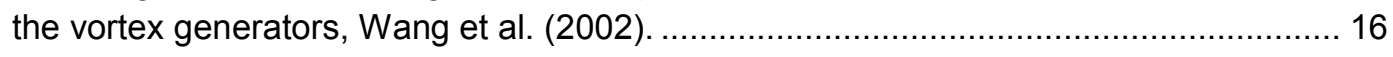

Figure 11 - Pictorial diagram of vortex generators, Zhou and Ye (2012) ....................................... 17

Figure 12 - Schematic of the large winglet and two kinds of delta winglet arrays geometry and placements, He et al. (2012)..................................................................................... 18

Figure 13 - The main domain of the CHE with two tubes and two delta winglet VGs........................ 20

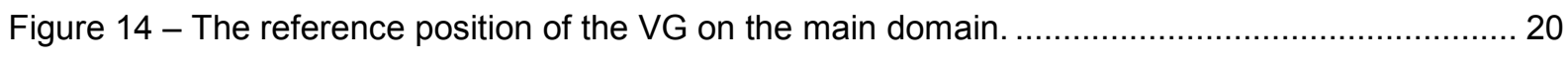

Figure 15 - VGS position attached to the bottom fin. ............................................................... 22

Figure 16 - (a) Overview of the main domain, Axis-x, (b) Highlight of the $V G_{1}$, (c) Highlight of the $V G_{2}$.

Figure 17 - (a) Overview of the main domain. Axis-z, (b) Highlight of the $V G_{1}$, (c) Highlight of the $V G_{2}$.

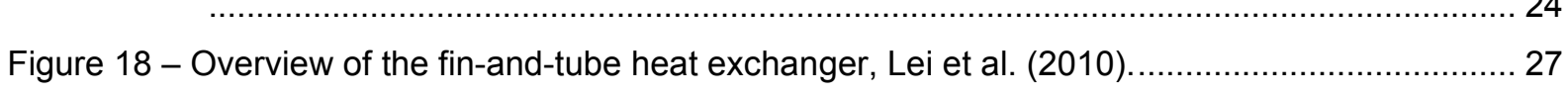

Figure 19 - Dimensions/position of the VGs and computational domain, Lei et al. (2010)................ 28

Figure 20 - Structure of longitudinal vortex $(\operatorname{Re}=1000)$ for aspect ratio 2, Lei et al. $(2010)$.............. 28

Figure 21 - Velocity vector plots on a plane near the fin $(R e=1000)$ for aspect ratio 2. (A) Plain fin. (B)

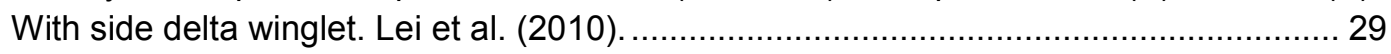

Figure 22 - Exit temperature field for aspect ratio 2. (A) Plain fin. (B) With VG. Lei et al. (2010)....... 30

Figure 23 - Fin surface temperature field for aspect ratio 2. (A) Plain fin. (B) With VG. Lei et al. (2010).

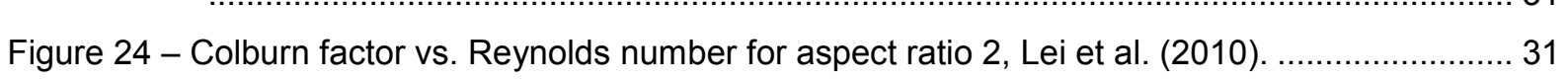

Figure 25 - Friction factor vs. Reynolds number for aspect ratio 2, Lei et al. (2010). ........................ 32

Figure 26 - Heat transfer and drag for different arrangements and aspect ratio 2, Lei et al. (2010)... 33

Figure 27 - Colburn factor at different attack angles and aspect ratio 2, Lei et al. (2010)................. 34

Figure 28 - Friction factor at different attack angles and aspect ratio 2, Lei et al. (2010)................... 34

Figure 29 - Performances at different attack angles and aspect ratio 2, Lei et al. (2010)................. 35

Figure 30 - Colburn factor at different aspect ratios for attack angle of $20^{\circ}$, Lei et al. (2010). ........... 36

Figure 31 - Friction factor at different aspect ratios for attack angle of $20^{\circ}$, Lei et al. (2010)............ 36 
Figure 32 - Performance at different aspect ratios for attack angle of $20^{\circ}$, Lei et al. (2010).

Figure 33 - Configuration of winglet type vortex generator on the fin surface-tube bank: (a) common-

flow-down configuration; (b) common-flow-up configuration, Torii et al. (2002).

Figure 34 - Five types of fin configurations, Tang et al. (2009).

Figure 35 - Geometric configuration of the delta-winglets in the elementary model used by Lemouedda et al. (2010)

Figure 36 - Time averaging of turbulence used on RANS models.

Figure 37 - Subdivisions of the Near-Wall Region. 64

Figure 38 - Two approaches to Near-Wall Treatments in Ansys Fluent (2010)...... 65

Figure 39 - Sequence of operations in SIMPLE algorithm. 70

Figure 40 - Typical control volume and the notation used for a Cartesian 2D grid. 74

Figure 41 - Illustration of the main computational domain and geometric parameters of the heat exchanger model studied. 76

Figure 42 - Computational domain including main domain and extended region. 78

Figure 43 - Flowchart for optimization considering surrogate model in two steps. ............................. 88

Figure 44 - General flowchart for direct optimization in one step. 88

Figure 45 - Schematic of a fully connected multilayer ANN, Kreith et al. (2000). 96

Figure 46 - Nodal operation in an ANN, Kreith et al. (2000). 98

Figure 47 - Probability density at $R e=250$ (a) Friction factor and, (b) Colburn factor. 114

Figure 48 - Probability density at $\operatorname{Re}=1400$ (a) Friction factor and, (b) Colburn factor. 115

Figure 49 - Contribution ratio of the main/interaction effect for Colburn factor (j) at $R e=250$. 121

Figure 50 - Contribution ratio of the main/interaction effect for Friction factor (f) at $\operatorname{Re}=250$. 121

Figure 51 - Contribution ratio of the main/interaction effect for Colburn factor (j) at $\operatorname{Re}=1400 \ldots \ldots . .123$

Figure 52 - Contribution ratio of the main/interaction effect for Friction factor (f) at $\operatorname{Re}=1400 \ldots \ldots . .123$

Figure 53 - Comparison of the contribution ratio of main/interaction effect for Colburn factor (j)..... 125

Figure 54 - Comparison of the contribution ratio of main/interaction effect for Friction factor (f)...... 125

Figure 55 - Global results for JF objective function. 131

Figure 56 - Global results for $\mathrm{JF}^{\frac{1}{3}}$ objective function. 131

Figure 57 - Global results for j objective function with correspondent drop pressure penalty. 132

Figure 58 - Global results for $f$ objective function with correspondent augmentation heat transfer... 132

Figure 59 - Overview of the position and angle for the best results from Figure 55 to Figure $58 \ldots . . .133$ Figure 60 - Span-average Colburn factor (j) for the best results (Figure 55) for objective function JF.

Figure 61 - Span-average Colburn factor (j) for the best results (Figure 56) for objective function $\mathrm{JF}^{\frac{1}{3}}$.

Figure 62 - Span-average Colburn factor (j) for the best results (Figure 57) for objective function j. 134 Figure 63 - Relation between span-average Colburn factor (j) with and without VG for the best results (Figure 55) for objective function JF.

Figure 64 - Relation between span-average Colburn factor (j) with and without VG for the best results

(Figure 56) for objective function $\mathrm{JF}^{\frac{1}{3}}$.

Figure 65 - Relation between span-average Colburn factor (j) with and without VG for the best results

(Figure 57) for objective function j. 
Figure 66 - Streamlines emitted from Core vortex by Torii et al. (2002) arrangement...................... 139

Figure 67 - Objective function JF. Streamlines emitted from Core vortex by present work (DO) ..... 140

Figure 68 - Velocity and temperature field planes at axis-z, according to Figure 55 ..................... 140

Figure 69 - Objective function JF. Flow separation profile on (a) tube 1, (b) tube 2 ....................... 141

Figure 70 - Pressure profile considering the objective function JF. ........................................... 141

Figure 71 - Streamlines emitted from Core vortex by Lemouedda et al. (2010) arrangement. ......... 143

Figure 72 - Objective function $\mathrm{JF}^{\frac{1}{3}}$. Streamlines emitted from Core vortex by present work (DO)... 143

Figure 73 - Velocity and temperature field planes at axis-z, according to Figure 56 ...................... 144

Figure 74 - Objective function $\mathrm{JF}^{\frac{1}{3}}$. Flow separation profile on (a) tube 1, (b) tube 2..................... 144

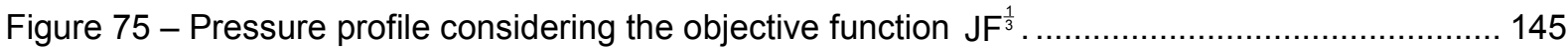

Figure 76 - Objective function j. Streamlines emitted from Core vortex by present work (DO). ........ 146

Figure 77 - Velocity and temperature field planes at axis-z, according to Figure 57 ...................... 147

Figure 78 - Objective function j. Flow separation profile on (a) tube 1, (b) tube 2......................... 147

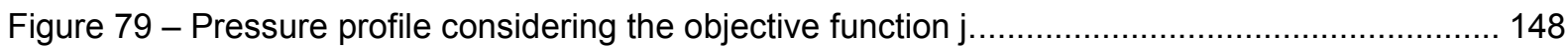

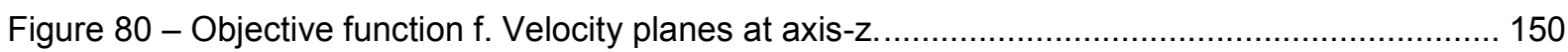

Figure 81 - Objective function f. Flow separation profile on (a) tube 1, (b) tube 2 ......................... 150

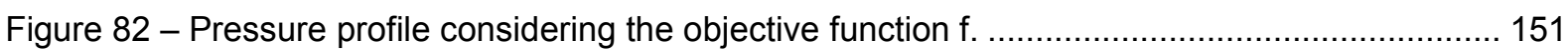

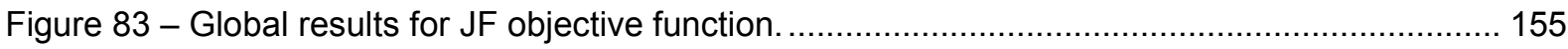

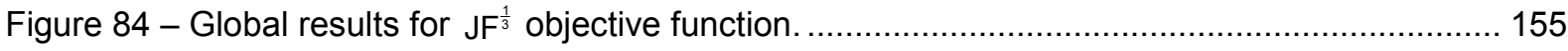

Figure 85 - Global results for j objective function with correspondent drop pressure penalty. .......... 155

Figure 86 - Global results for $f$ objective function with correspondent augmentation heat transfer. .. 156

Figure 87 - Overview of the position and angle for the best results from Figure 83 to Figure $86 \ldots . . .156$

Figure 88 - Comparison between vortical structures by DO and RSM approach for objective function j.

Figure 89 - Span-average Colburn factor (j) for the best results (Figure 83) for objective function JF.

Figure 90 - Span-average Colburn factor (j) for the best results (Figure 84) for objective function $\mathrm{JF}^{\frac{1}{3}}$.

Figure 91 - Span-average Colburn factor (j) for the best results (Figure 85) for objective function j. 158 Figure 92 - Objective function JF. Span-average Colburn factor (j) for the best results (Figure 83). 162 Figure 93 - Objective function $\mathrm{JF}^{\frac{1}{3}}$. Span-average Colburn factor (j) for the best results (Figure 84).

Figure 94 - Objective function j. Span-average Colburn factor (j) for the best results (Figure 85). ... 162

Figure 95 - Streamlines emitted from Core vortex by Torii et al. (2002) arrangement....................... 163

Figure 96 - Objective function JF. Streamlines emitted from Core vortex by present work (DO) ..... 164

Figure 97 - Velocity and temperature field planes at axis-z, according to Figure 83...................... 164

Figure 98 - Objective function JF. Flow separation profile on (a) tube 1, (b) tube 2........................ 165

Figure 99 - Pressure profile considering the objective function JF. ............................................. 165

Figure 100 - Streamlines emitted from Core vortex by Lemouedda et al. (2010) arrangement. ....... 167

Figure 101 - Objective function $\mathrm{JF}^{\frac{1}{3}}$. Streamlines emitted from Core vortex by present work (DO).. 168 
Figure 102 - Velocity and temperature field planes at axis-z, according to Figure 84 168

Figure 103 - Objective function $\mathrm{JF}^{\frac{1}{3}}$. Flow separation profile on (a) tube 1, (b) tube 2 . 169

Figure 104 - Pressure profile considering the objective function $\mathrm{JF}^{\frac{1}{3}}$. 169

Figure 105 - Objective function j. Streamlines emitted from Core vortex by present work (DO)...... 171

Figure 106 - Velocity and temperature field planes at axis-z, according to Figure $85 . \ldots \ldots \ldots \ldots \ldots \ldots 17$

Figure 107 - Objective function j. Flow separation profile on (a) tube 1, (b) tube 2. .................... 172

Figure 108 - Pressure profile considering the objective function j........................................ 172

Figure 109 - Velocity and temperature field planes at axis-z, according to Figure 86...................174

Figure 110 - Objective function f. Flow separation profile on (a) tube 1, (b) tube $2 \ldots \ldots \ldots \ldots \ldots \ldots \ldots . . . \ldots \ldots$

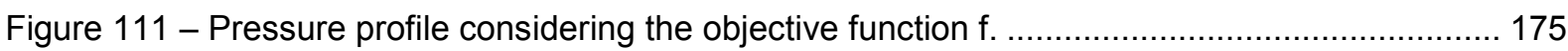

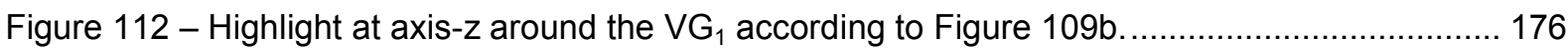

Figure 113 - Comparison between vortex generator arrangements for different Reynolds number found by optimizer for each objective functions.......................................... 179

Figure 114 - Vortex generator arrangements by present work (DO) for all objective functions........ 179

Figure 115 - Comparison among global results for each objective function at $\operatorname{Re}=250$.............. 180

Figure 116 - Comparison among global results for each objective function at $\operatorname{Re}=1400 . \ldots \ldots \ldots \ldots 1$

Figure 117 - Friction factor from Wang et al. (2000) correlation - Equation 87 - and the different numerical approaches. Without vortex generator.

Figure 118 - Colburn factor from Wang et al. (2000) correlation - Equation 88 - and the different numerical approaches. Without vortex generator.

Figure 119 - Impact on inlet turbulence intensity in Colburn and Friction factor for $\operatorname{Re}=250 \ldots \ldots \ldots 204$

Figure 120 - Impact on inlet turbulence intensity in Colburn and Friction factor for $\operatorname{Re}=1400 \ldots \ldots . .204$

Figure 121 - Results for $\mathrm{Re}=250$ and SST k-omega turbulence model. (a) Heat transfer coefficient,

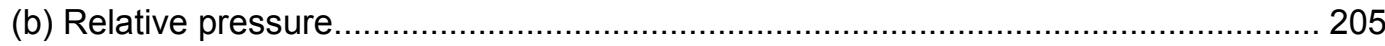

Figure 122 - Results for $\mathrm{Re}=1400$ and SST k-omega turbulence model. (a) Heat transfer coefficient, (b) Relative pressure.

Figure 123 - RSM training error and regression as a function of the number of hidden neurons unit at

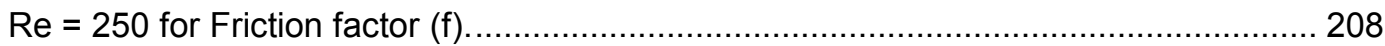

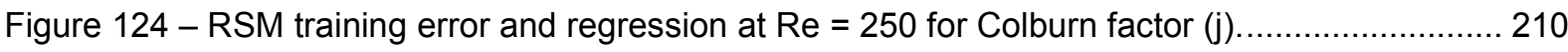

Figure 125 - RSM training error and regression at $\mathrm{Re}=1400$ for Friction factor (f).......................2 211

Figure 126 - RSM training error and regression at $\mathrm{Re}=1400$ for Colburn factor (j) .................... 212

Figure 127 - Regression and error training comparison for different meta-modeling method at $\operatorname{Re}=250$

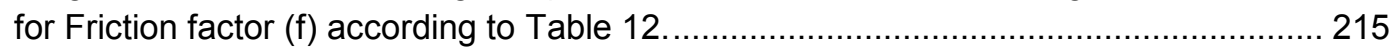

Figure 128 - Regression and error training comparison for different meta-modeling method at $\operatorname{Re}=250$ for Colburn factor (j) according to Table 12.

Figure 129 - Regression and error training comparison for different meta-modeling method at $\mathrm{Re}=1400$ for Friction factor (f) according to Table 12 .

Figure 130 - Regression and error training comparison for different meta-modeling method at $\mathrm{Re}=1400$ for Colburn factor (f) according to Table 12 . 


\section{LIST OF TABLES}

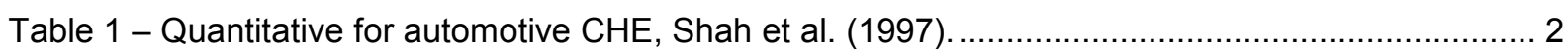

Table 2 - Parameters ranges applied to meta-modeling and optimization..................................... 25

Table 3 - Commonly turbulence models, Versteeg and Malalasekera (2007)............................... 48

Table 4 - Comparison of computer resources and solver performance for the RAE 2822 airfoil. ....... 68

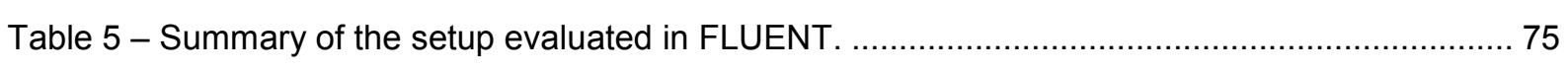

Table 6 - Geometric dimensions of heat exchanger model................................................. 76

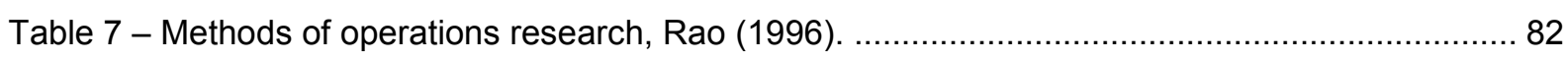

Table 8 - Position and angle according to reference adopted in present work............................. 128

Table 9 - Summary of the grid independence without vortex generator. ................................... 202

Table 10 - Summary of the grid independence with vortex generator for $\mathrm{Re}=250 . \ldots \ldots \ldots \ldots \ldots \ldots . \ldots 2$

Table 11 - Summary of the grid independence with vortex generator for $\operatorname{Re}=1400 \ldots \ldots \ldots \ldots \ldots \ldots . \ldots 2$

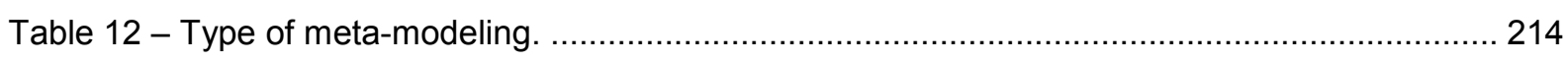




\section{LIST OF ABBREVIATIONS AND ACRONYMS}

\begin{tabular}{ll} 
Al & Artificial Intelligence \\
ANN & Artificial Neural Network \\
BHE & Brazed Heat Exchanger \\
CFD & Computational Fluid Dynamic \\
CHE & Compact Heat Exchanger \\
CNT & Carbon Nanotubes \\
CPU & Central Processing Unit \\
DNS & Direct Numerical Simulation \\
DO & Direct Optimization \\
DoE & Design of Experiment \\
EWT & Enhanced Wall Treatment \\
GA & Genetic Algorithm \\
HE & Heat Exchanger \\
LES & Large Eddy Simulation \\
LHS & Latin Hypercube Sampling \\
LVG & Longitudinal Vortex Generator \\
MHE & Mechanical Heat Exchanger \\
MMC & Metal Matrix Composite \\
NN & Neural Network \\
RANS & Reynolds Averaged Navier-Stokes \\
RBF & Radial Basis Functions \\
RNG & ReNormalization Group Theory \\
RSM & Response Surface Methodology \\
SST & Shear-Stress Transport \\
TVG & Transverse Vortex Generator \\
HTE & Heat Transfer Enhanced \\
VG & Vortex Generator \\
VGCF & Vapor Grown Carbon Fiber \\
\hline
\end{tabular}




\section{LIST OF SYMBOLS}
$A_{c}$
Minimum flow area $\left[\mathrm{mm}^{2}\right]$
$A_{\circ}$
Wet total surface area of the HE $\left[\mathrm{mm}^{2}\right]$
$\Lambda$
Aspect ration $[a / b]$
a
Length of the VG [mm]
b
Height of the VG [mm]
$\mathrm{C}_{\mathrm{p}}$
Specific heat $[\mathrm{J} / \mathrm{kg} . \mathrm{K}]$
$D_{0}$
Diameter of tube [mm]
$D_{c}$
Fin collar outside diameter [mm]
$\Delta p$
Pressure loss $[\mathrm{Pa}]$
$\Delta \mathrm{T}$
Log-mean temperature difference $[\mathrm{K}]$
$\delta_{\mathrm{f}}$
Fin thickness [mm]
$\rho$
Density $\left[\mathrm{kg} / \mathrm{m}^{3}\right]$
$\Gamma_{\phi}$
Diffusion coefficient
$\mathrm{e}_{\max }$
Maximum relative error
$\mathrm{e}_{\text {mean }}$
Mean relative error
f
Friction factor
$\mathrm{F}_{\mathrm{P}}$
Fin pitch [mm]
$f\left(r_{t 1}^{2}\right)$
Constant as function of the radius of the tube 1.
$f\left(r_{t 2}^{2}\right)$
Constant as function of the radius of the tube 2 .
$\tilde{\phi}_{\mathrm{i}}$
Predicted value by RSM
$\bar{\phi}_{\mathrm{i}}$
Mean of all real value from training data set
$\phi_{i}^{\mathrm{NORM}}$
Normalized generic variable
$\phi$
Generic variable
$\phi_{\mathrm{i}}$
Real value of output from training data set
$\varphi_{1}$
Roll angle of the $V G_{1}$ at axis-x $\left[{ }^{\circ}\right]$ 


\begin{tabular}{|c|c|}
\hline$\varphi_{2}$ & Roll angle of the $\vee G_{2}$ at axis-x $\left[^{\circ}\right]$ \\
\hline $\mathrm{h}$ & Heat transfer coefficient $\left[\mathrm{W} / \mathrm{m}^{2} . \mathrm{K}\right]$ \\
\hline IQR & Interquartille range $\left(Q_{1}-Q_{3}\right)$ \\
\hline I & Turbulent intensity \\
\hline j & Colburn factor \\
\hline $\mathrm{J}_{1}$ & First node number in NN input \\
\hline$J_{1}$ & Last node number in NN input \\
\hline $\mathrm{k}_{\mathrm{f}}$ & Thermal conductivity of fluid $[\mathrm{w} / \mathrm{m} . \mathrm{K}]$ \\
\hline $\mathrm{L}$ & Depth of the CHE [mm] \\
\hline $\mathrm{N}$ & Number of the tube row \\
\hline $\mathrm{N}_{\mathrm{t}}$ & Number of training data set \\
\hline$N_{n}$ & Total number of internal nods in NN \\
\hline $\operatorname{Pr}$ & Prandtl number \\
\hline $\mathrm{P}_{\mathrm{T}}$ & Transverse pitch [mm] \\
\hline$P_{L}$ & Longitudinal pitch [mm] \\
\hline Q & Total heat transfer [W] \\
\hline$Q_{1}$ & First or lower quartile \\
\hline$Q_{3}$ & Third or upper quartile \\
\hline$r_{\mathrm{t} 1}$ & Radius of the tube $1[\mathrm{~mm}]$ \\
\hline$r_{\mathrm{t} 2}$ & Radius of the tube $2[\mathrm{~mm}]$ \\
\hline $\operatorname{Re}$ & Reynolds number \\
\hline $\mathrm{Re}_{\mathrm{co}}$ & Reynolds number based on fin collar outside diameter \\
\hline $\mathrm{R}^{2}$ & Coefficient of determination of a linear regression \\
\hline$S_{\max }$ & Maximum value of individual input/output variable \\
\hline $\mathrm{S}_{\min }$ & Minimum value of individual input/output variable \\
\hline $\mathrm{S}_{\phi}$ & Source term \\
\hline$\theta_{1}$ & Attack angle of the $V G_{1}$ at axis- $z\left[^{\circ}\right]$ \\
\hline$\theta_{2}$ & Attack angle of the $V G_{2}$ at axis-z $\left[^{\circ}\right]$ \\
\hline $\mathrm{T}$ & Temperature $[\mathrm{K}]$ \\
\hline
\end{tabular}




\begin{tabular}{|c|c|}
\hline $\mathrm{T}_{\text {wall }}$ & Temperature of the tube $[\mathrm{K}]$ \\
\hline $\mathrm{T}_{\mathrm{s}}$ & Superficial temperature $[\mathrm{K}]$ \\
\hline$T_{\text {ref }}$ & Reference temperature $[\mathrm{K}]$ \\
\hline $\mathrm{T}_{\text {inlet }}$ & Temperature at inlet domain $[\mathrm{K}]$ \\
\hline $\mathrm{u}_{\text {inlet }}$ & Velocity at inlet domain $[\mathrm{m} / \mathrm{s}]$ \\
\hline $\mathrm{u}_{\mathrm{c}}$ & Velocity at minimum flow area $[\mathrm{m} / \mathrm{s}]$ \\
\hline$V G_{1}$ & Vortex Generator of the tube 1 \\
\hline $\mathrm{VG}_{2}$ & Vortex Generator of the tube 2 \\
\hline$x_{1}$ & Position of the $V G_{1}$ at axis-x $[\mathrm{mm}]$ \\
\hline $\mathrm{x}_{2}$ & Position of the $V G_{2}$ at axis- $x[\mathrm{~mm}]$ \\
\hline $\mathrm{y}_{1}$ & Position of the $V G_{1}$ at axis-y [mm] \\
\hline $\mathrm{y}_{2}$ & Position of the $V \mathrm{G}_{2}$ at axis-y $[\mathrm{mm}]$ \\
\hline$x_{t 1}$ & Center point of the tube 1 at axis- $x[\mathrm{~mm}]$ \\
\hline$x_{t 2}$ & Center point of the tube 2 at axis- $x[\mathrm{~mm}]$ \\
\hline$y_{t 1}$ & Center point of the tube 1 at axis-y $[\mathrm{mm}]$ \\
\hline$y_{\mathrm{t} 2}$ & Center point of the tube 2 at axis-y $[\mathrm{mm}]$ \\
\hline u & NN weight among input, hidden layer and output \\
\hline
\end{tabular}




\section{TABLE OF CONTENTS}

\section{Chapter 1}

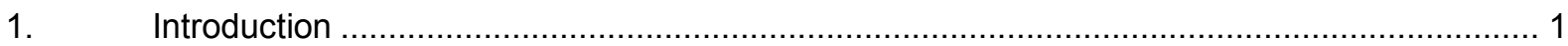

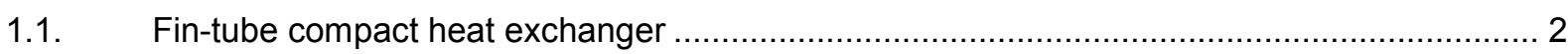

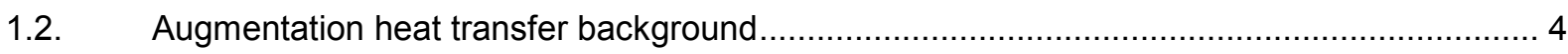

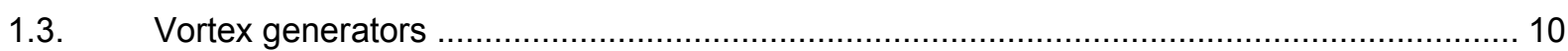

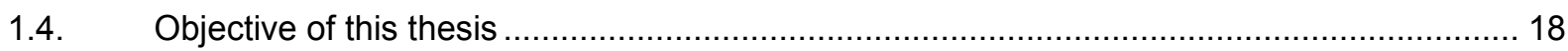

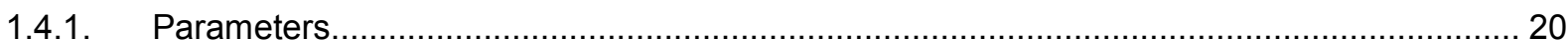

\section{Chapter 2}

2. Literature review on heat transfer enhancement by longitudinal vortex generators (LVG) ... 26

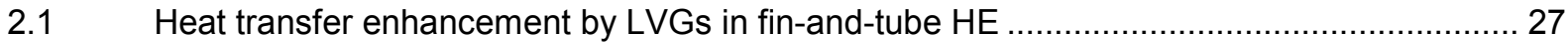

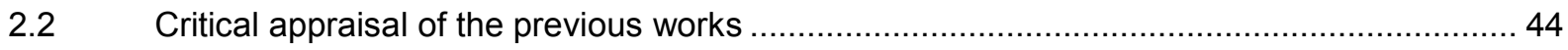

\section{Chapter 3}

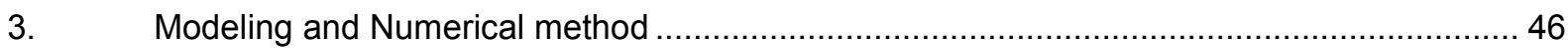

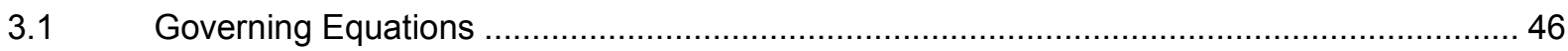

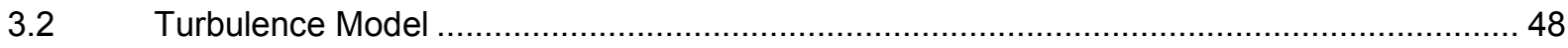

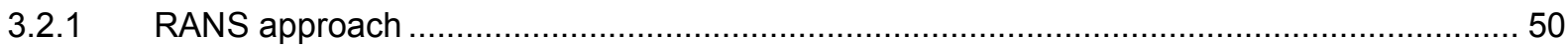

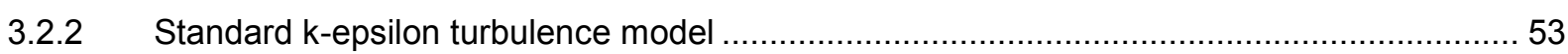

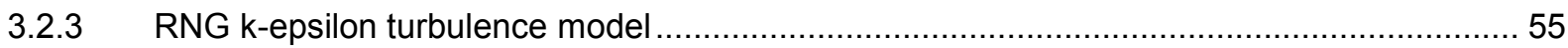

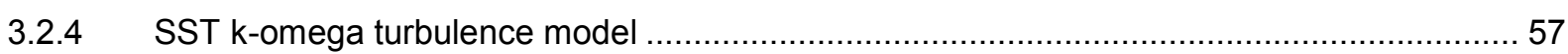

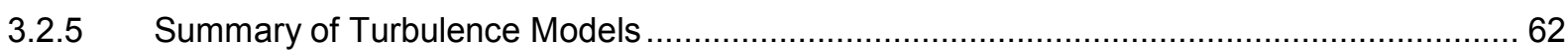

3.3 Near-Wall Treatments for Wall-Bounded Turbulent Flows .............................................. 63

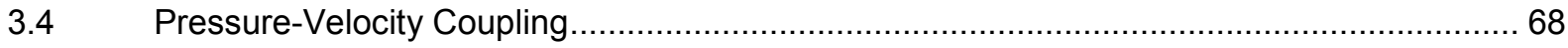

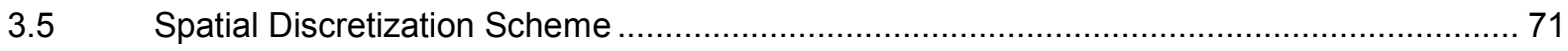

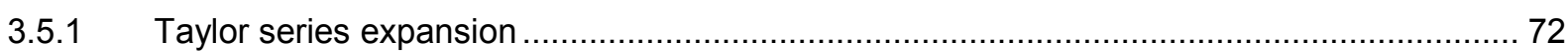

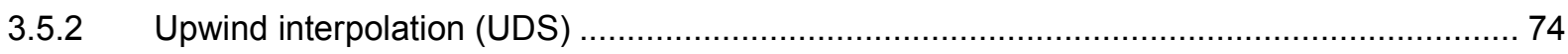

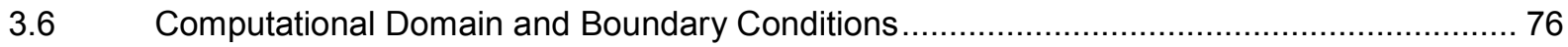

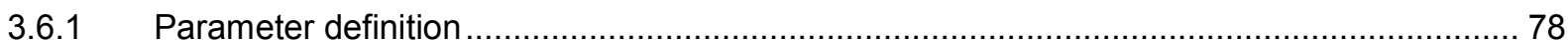

\section{Chapter 4}

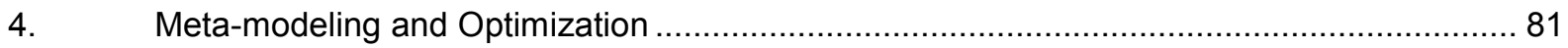

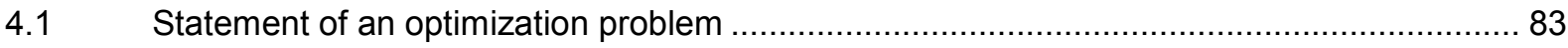




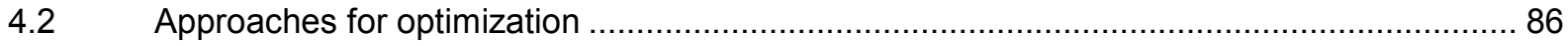

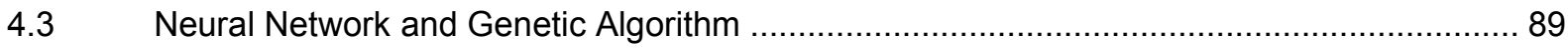

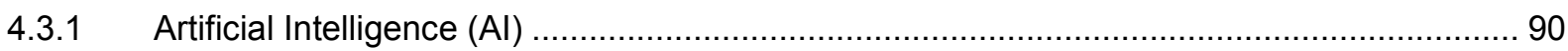

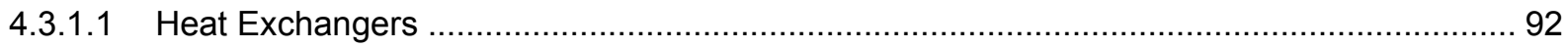

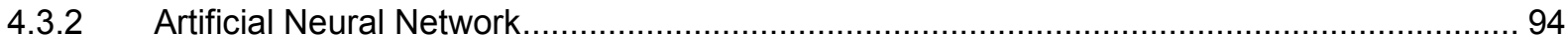

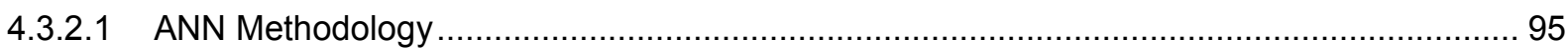

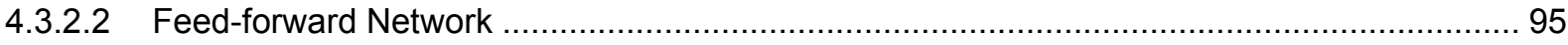

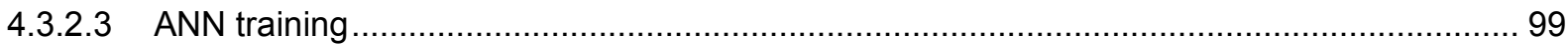

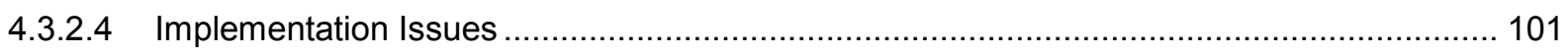

4.3.2.5 Additional Applications in Thermal Engineering …...................................................... 104

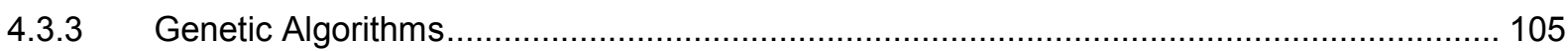

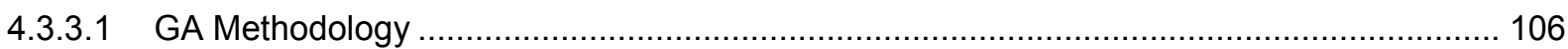

4.3.3.2 Additional Applications in Thermal Engineering …................................................... 108

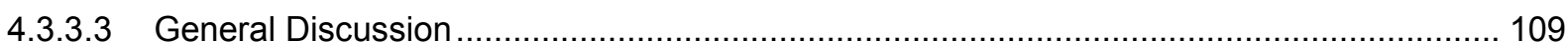

4.4 Design of Experiment (DoE) and RSM training ........................................................ 110

\section{Chapter 5}

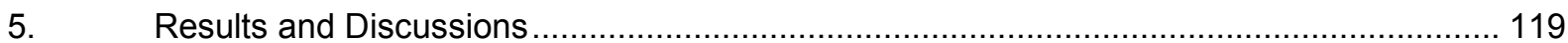

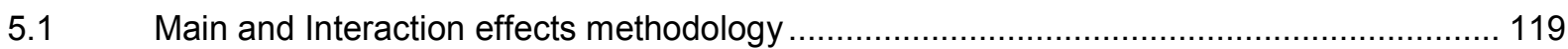

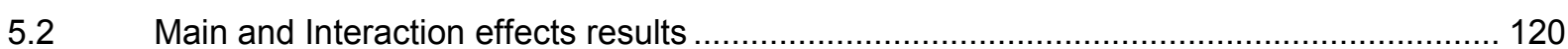

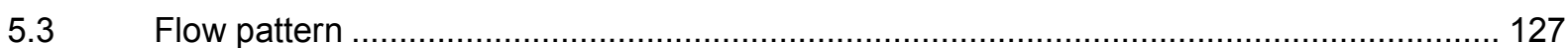

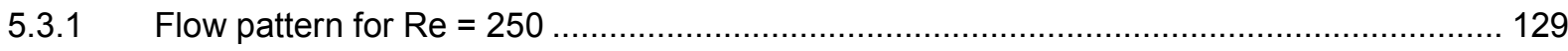

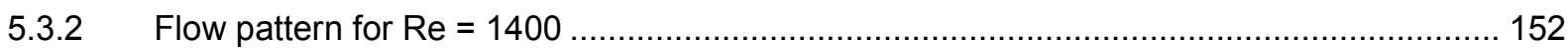

\section{Chapter 6}

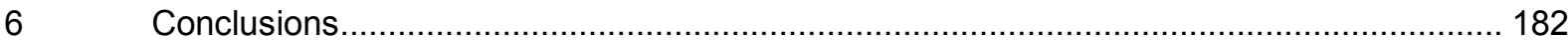

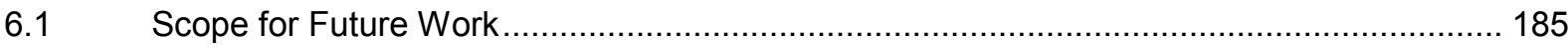

\section{Appendix A}

A. CFD validation procedure 198

\section{Appendix B}

B. Surrogate model validation 


\section{Introduction}

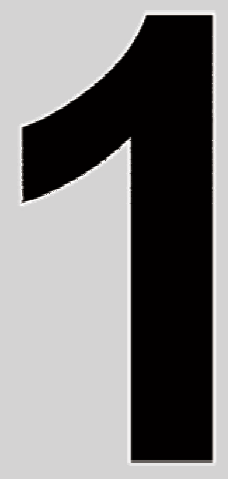

HE are used either individually or as components of a large thermal system in a wide variety of commercial, industrial and household applications, eg, power generation, refrigeration, ventilating and air-conditioning systems, process, manufacturing, aerospace and automotive industries, electronic chip cooling, as well as, in environmental engineering.

The improvements in the performance of the HE have attracted many researchers for a long time as they are of great technical, economical, and not the least, ecological importance. Performance improvement becomes essential particularly in HE with gases because the thermal resistance of gases can be 10 to 50 times as large as that of liquids (TIGGELBECK et al., 1992), which requires large heat transfer surface area per unit volume on the gas side. A large ratio area per unit volume defines a class of HE called by Compact Heat Exchanger (CHE). This type of HE is discussed in Section 1.1.

The traditional methods of reducing the air-side thermal resistance are either increasing the surface area of the HE or reducing the thermal boundary layer thickness on the surface of the HE. Increasing the surface area is effective but results in the increase of material cost and mass of the heat exchanger (an easier way to make it is increase the fin density of HE). This approach becomes unfeasible some application especially those which have not enough physical space, such as: automotive projects (radiator, intercooler, condenser, oil-cooler, etc.). 
Several methodologies are presented here (Section 1.2), only as an overview, but the main goal of this thesis is to explore the second method mentioned previously that reduces boundary layer thickness by generation of passive vortices. In this technique the flow field is altered by an obstacle that produces an oriented vortex in the direction of the flow. This changes the flow and, consequently, alters the local hydrodynamic and thermal boundary layer. The net effect of this manipulation is an average increase in the heat transfer, usually associated with penalty in pressure drop. This technique is discussed in Section 1.2 and Section 1.3.

Overall, the present work is undertaken to compute the enhancement heat transfer levels achievable in a fin-tube HE, with built-in vortex generators (VG) mounted on fins by meta-modeling and optimization process, through Neural-Network (NN) and Genetic Algorithm (GA), respectively.

\subsection{Fin-tube compact heat exchanger}

Compact heat exchangers ( $\mathrm{CHE}$ ) are characterized by a large heat transfer surface area per unit volume. HE is referred to as a $\mathrm{CHE}$ if its area density is greater than $700 \mathrm{~m}^{2} / \mathrm{m}^{3}$. For example, Table 1 shows the typical quantitative of the CHE applied in automotive projects.

Table 1 - Quantitative for automotive CHE, Shah et al. (1997).

\begin{tabular}{ccccc}
\hline HE & $\begin{array}{c}\text { Compactness } \\
\mathrm{m}^{2} / \mathrm{m}^{3}\end{array}$ & $\begin{array}{c}\text { Operating Pressure } \\
\text { bar }\end{array}$ & $\begin{array}{c}\text { Operating Temperature } \\
{ }^{\circ} \mathrm{C}\end{array}$ & $\begin{array}{c}\text { Mass } \\
\mathrm{kg}\end{array}$ \\
\hline Radiator & $1000-1500$ & $1.6-2.5$ & $80-125$ & $1.5-5.0$ \\
Condenser & $950-1300$ & $15-23$ & $75-100$ & $1.2-4.0$ \\
Heater & $1800-2800$ & $1.6-2.5$ & $80-125$ & $0.5-1.2$ \\
Evaporator & $900-1000$ & $3-3.8$ & $3-7$ & $1.2-3.5$ \\
Oil Cooler & $500-1500$ & $3-10$ & $120-150$ & $0.3-1.5$ \\
Charge Air Cooler & $600-900$ & $2-3.5$ & $120-200$ & $0.6-3.5$ \\
\hline
\end{tabular}

Fin-tube CHE consists of many diverse tube, fin, and flow orientations, Figure 1. Generally, fin-tube CHE are comprised of small bore tubes spaced closely together. According to Shah et al. (1981), fin-tube CHE can be up to $3300 \mathrm{~m}^{2} / \mathrm{m}^{3}$ on the fin side. Fin-tube types can be consulted in details Incropera and De Witt (2002). 


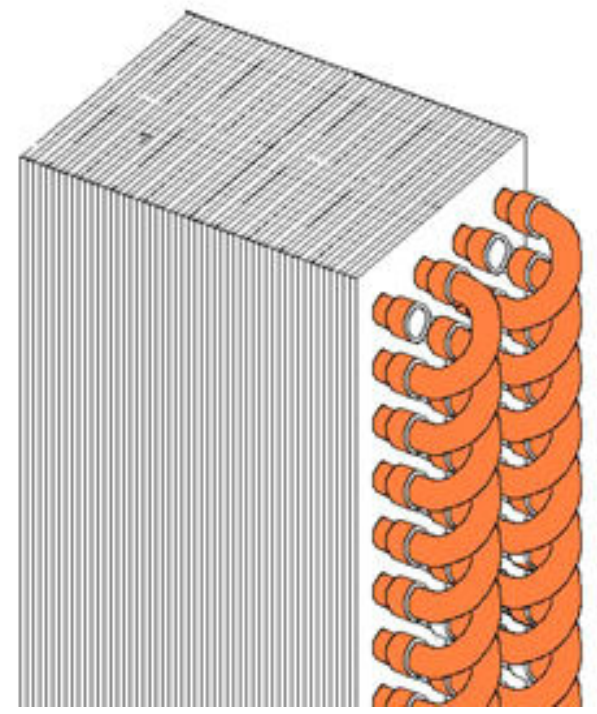

(a)

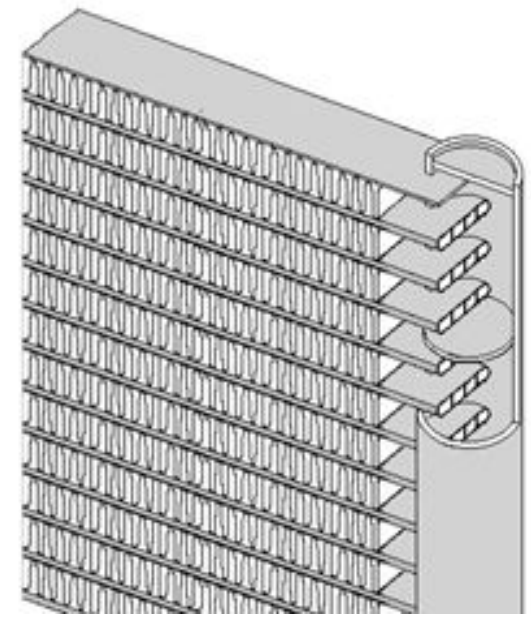

(b)

Figure 1 - Fin-tube CHE. (a) Mechanical Heat Exchanger (MHE), (b) Brazed Heat Exchanger (BHE).

Considering automotive projects, the Mechanical Heat Exchanger (MHE) in Figure 1(a) has been gradually replaced by another type of CHE called by Brazed Heat Exchanger (BHE), Figure 1(b). The main technical reason for these are:

1. BHE thermal efficiency is higher than MHE (with an identical front surface on the airside).

2. A lower depth of the BHE gives up to $50 \%$ lower pressure drop on the air side.

BHE thermal efficiency can be explained for increases in area surface (compactness is increased) and for enhancement heat transfer inside tubes due to baffles and/or turbulence protuberance. The lower pressure drop is due to lower depth; however, this depends on the fin density for each $\mathrm{CHE}$. As the released thermal energy by new engines is growing over the years, the BHE has become more suitable. On the other hand, the cost of $\mathrm{BHE}$ is significantly higher than $\mathrm{MHE}$, because of the manufacturing process. This is one more argument to keeping the studies in enhancement heat transfer in fin-tube $\mathrm{CHE}$, especially considering the configuration MHE. 


\subsection{Augmentation heat transfer background}

Bergles (1998) and (2001) classified the mechanisms of enhancing heat transfer as active or passive methods. Webb (2005) is also an excellent reference for it. The passive techniques generally use surface or geometrical modifications to the flow channel by incorporating inserts or additional devices. They promote higher heat transfer coefficients by disturbing or altering the existing flow behavior (except for extended surfaces) which also leads to increase in the pressure drop. In case of extended surfaces, effective heat transfer area on the side of the extended surface is increased. Passive techniques hold the advantage over the active techniques as they do not require any direct input of external power, rather they use from the system itself which ultimately leads to an increase in fluid pressure drop.

Hagge and Junkhan (1975) described various active mechanical enhancing methods that can be used to enhance heat transfer. These techniques are more complex from the use and design point of view as the method requires some external power input to cause the desired flow modification and improvement in the rate of heat transfer. This finds limited application because of the need of external power in many practical applications. In comparison to the passive techniques, these techniques have not shown much potential due to the difficult to provide external power input in many cases, Siddique et al. (2010). In these cases, external power is used to facilitate the desired flow modification and the concomitant improvement in the heat transfer.

Otherwise, the passive enhancement methods are those which do not require external power to sustain the enhancement characteristics. Examples of passive enhancing methods are: treated surfaces, rough surfaces, extended surfaces, displaced enhancement devices, swirl flow devices, coiled tubes, surface tension devices, additives for fluids, and many others.

According to Siddique et al. (2010), the mechanisms of heat transfer enhancement can be at least one of the following:

1. Use of a secondary heat transfer surface;

2. Disruption of the unenhanced fluid velocity; 
3. Disruption of the laminar sublayer in the turbulent boundary layer;

4. Introducing secondary flows;

5. Promoting boundary-layer separation;

6. Promoting flow attachment/reattachment;

7. Enhancing effective thermal conductivity of the fluid under static conditions;

8. Enhancing effective thermal conductivity of the fluid under dynamic conditions;

9. Delaying the boundary layer development;

10. Thermal dispersion;

11. Increasing the order of the fluid molecules;

12. Redistribution of the flow;

13. Modification of property of the convective medium;

14. Increasing the difference between the surface and fluid temperatures;

15. Increasing fluid flow rate passively;

16. Increasing the thermal conductivity of the solid phase using special nanotechnology fabrications.

Methods using mechanisms n.1 and n.2 include increasing the surface area in contact with the fluid to be heated or cooled by using fins, intentionally promoting turbulence in the wall zone employing surface roughness and tall/short fins, and inducing secondary flows by creating swirl flow through the use of helical/spiral fin geometry and twisted tapes, Figure 2. This tends to increase the effective flow length of the fluid through the tube, which increases heat transfer but also the pressure drop. However, for internal helical fins, the effect of swirl tends to decrease or vanish all together at higher helix angles, since the fluid flow simply passes axially over the fins, Li et al. (1982). For twisted tape inserts, the main contribution to the heat transfer augmentation is due to the effect of the induced swirl. 
(a)

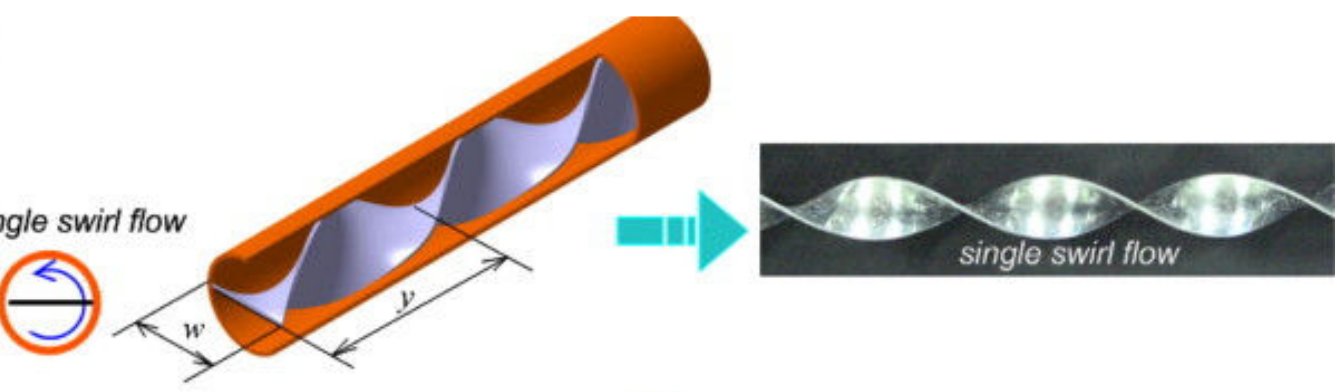

(b)

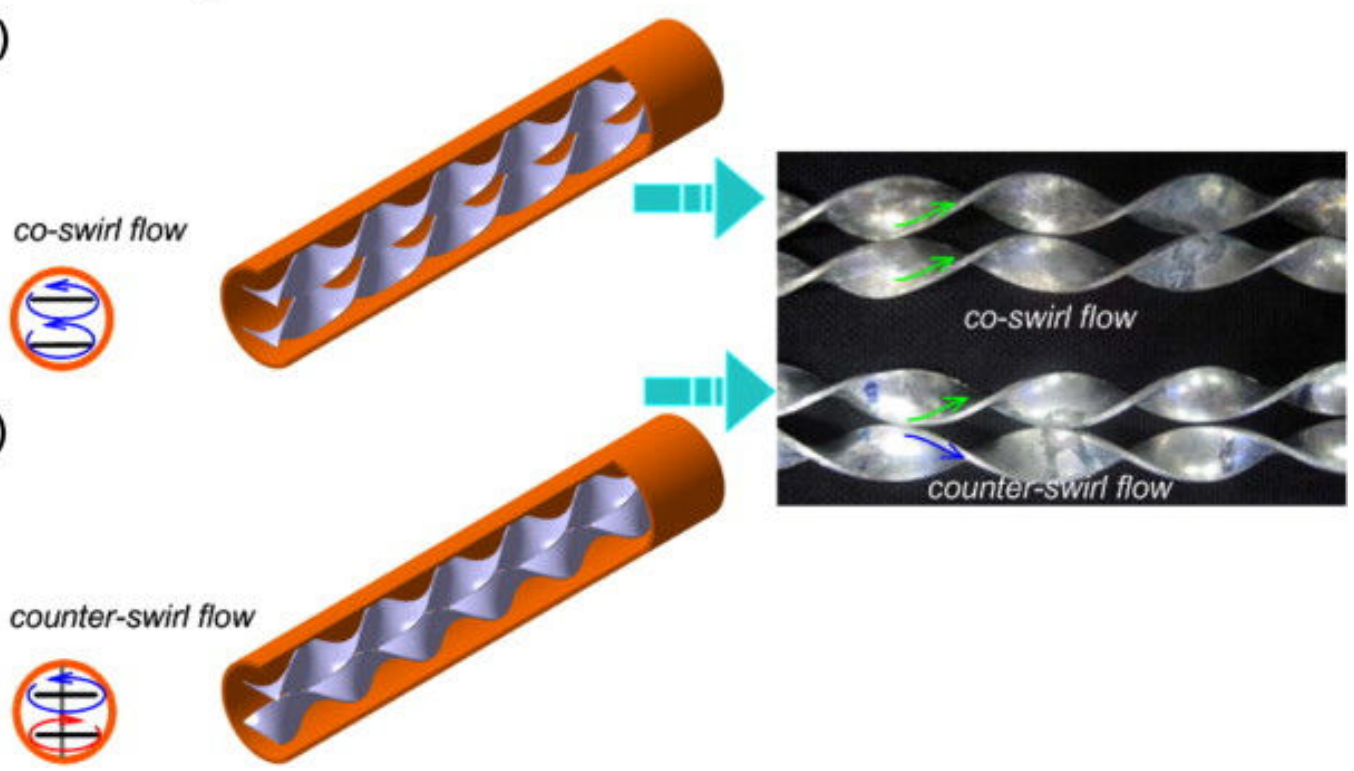

Figure 2 - Example of twisted tapes, (a) single swirl flow, (b) co-swirl flow and (c) counter-swirl flow, Eiamsa-Ard et al. (2010).

Turbulent flow in a tube exhibits a low-velocity flow region immediately adjacent to the wall, known as laminar sublayer (Figure 37), with velocity approaching zero at the wall. Most of the thermal resistance occurs in this low velocity region. Any roughness or enhancement technique that disturbs the laminar sublayer could enhance the heat transfer, Kohler and Staner (1984). For example, in a smooth tube of $25.4 \mathrm{~mm}$ inside diameter, at $\operatorname{Re}=30,000$, the laminar sublayer thickness is only $0.0762 \mathrm{~mm}$ under fully developed flow conditions. The internal roughness of the tube surface is known to increase the turbulent heat transfer coefficient. Therefore, for this example, an enhancement technique employing a roughness or fin element of height $\sim 0.07 \mathrm{~mm}$ disrupt the laminar sublayer and, thus, enhance the heat transfer. Therefore, mechanism n. 3 is a particularly important mechanism for augmenting heat transfer.

Mechanisms n.4 and n.5 can fit into the work developed by Li et al. (1982). They described the flow structure in helically finned tubes using flow visualization by 
means of high-speed photography employing the hydrogen bubble technique. They used four tubes with rounded ribs having helix angles between $38^{\circ}$ and $80^{\circ}$ and one or three fin starts. Photographs taken by them, showed that in laminar flow bubbles follow parabolic patterns, whereas in the turbulent flow these patterns break down because of random separation vortices. Also, for tubes with helical ridges, transition to turbulent flow is observed at lower Reynolds numbers compared to smooth tube values. Although swirl flow is observed for all tubes in the turbulent flow regime, the effect of the swirl is observed to decrease at higher helix angles. Li et al. (1982) concluded that both spiral and boundary-layer separation flow occur in helical tubes, but with different intensities in tubes having different configurations.

Mechanism n.6 plays an important role in heat transfer enhancements. About this, Arman and Rabas (1992) discussed the turbulent flow structure as the flow passes over a two-dimensional transverse rib. They identified the various flow separation and reattachment/redevelopment regions as:

- A small recirculation region in front of the rib;

- A recirculation region after the rib;

- A boundary layer reattachment/redevelopment region on the downstream surface, and finally;

- Flow up and over the subsequent rib.

The authors noting that recirculation eddies are formed above these flow regions, identified two peaks that occur in the local heat transfer, one at the top of the rib and the other in the downstream recirculation zone just before the reattachment point.

Heat transfer enhancements associated with fully/partially filing the fluidic volume by the porous medium, n.7, take place by the following mechanisms, Amiri and Vafai (1994) and Mohamad (2003):

- Enhancing effective thermal conductivity of the fluid under static conditions;

- Enhancing effective thermal conductivity of the fluid under dynamic conditions; 
- Delaying the boundary layer development;

- Thermal dispersion;

- Increasing the order of the fluid molecules;

- Redistribution of the flow;

- Modification of property of the convective medium.

Ding et al. (2006) showed that fluids containing $0.5 \mathrm{wt} . \%$ of carbon nanotubes (CNT) can produce heat transfer enhancements over $250 \%$ at $\operatorname{Re}=800$, and the maximum enhancement occurs at an axial distance of approximately 110 times the tube diameter. These types of mixtures are named in the literature as Nanofluids. Ahmeda et al. (2012) make an excellent review about this technique. Nanofluids are fluids that contain suspensions of nanoparticles of high thermally conductive materials like carbon, metals, and metal oxides into heat transfer fluids to improve the overall thermal conductivity. These nanoparticles are usually of order $100 \mathrm{~nm}$ or less. The increases in heat transfer due to presence of nanofluids are associated with the following mechanisms.

- Enhancing effective thermal conductivity of the fluid under static conditions.

- Enhancing effective thermal conductivity of the fluid under dynamic conditions.

- Delaying the boundary layer development.

- Thermal dispersion.

- Increasing the order of the fluid molecules.

- Redistribution of the flow.

The advantages of properly engineered nanofluids include the following: 
- Higher thermal conductivities than that predicted by currently available macroscopic models;

- Excellent stability;

- Little penalty due to an increase in pressure drop, and;

- Little penalty due to an increase in pipe wall abrasion experienced by suspensions of millimeter or micrometer particles.

Flexible fluidic thin films were introduced in the work of Khaled and Vafai (2002-2003) and Khaled (2007). In their works, they describe a new passive method for enhancing the cooling capability of fluidic thin films. In summary, flexible thin films utilize soft seals to separate between their plates instead of having rigid thin film construction. Khaled and Vafai (2002) have demonstrated that more cooling is achievable when flexible fluidic thin films are utilized. The expansion of the flexible thin film including flexible microchannel heat sink is directly related to the average internal pressure inside the microchannel. Additional increase in the pressure drop across the flexible micro channel not only increases the average velocity, but also expands the microchannel causing an apparent increase in the coolant flow rate which, in turn, increases the cooling capacity of the thin film. Khaled and Vafai (2003) and Khaled (2007) have demonstrated that the cooling effect of flexible thin films can be further enhanced, if the supporting soft seals contain closed cavities filled with a gas, which is in contact with the heated plate boundary of the thin film. They referred to this kind of sealing assembly as "flexible complex seals". The resulting fluidic thin film device is expandable according to an increase in the working internal pressure or an increase in the heated plate temperature. Therefore, mechanism n.15 impacts in enhancing heat transfer in thermal systems. The mechanism n.14 finds applications when rooted fins are utilized as an enhancer of heat transfer, Khaled (2008).

Heat transfer enhancement using Ultra High Thermal Conductivity Composite Materials is applied to increase the thermal conductivity of the solid phase using special nanotechnology fabrications, mechanism n.16. Composite materials have been used primarily for structural applications. However, they found to be useful for 
heat dissipation especially in electronic devices. An example of such these materials is the metal matrix composite (MMC). Typical MMCs that includes aluminum and copper matrix composites did not show substantial improvements in thermal conductivity, except when reinforcing agent of vapor grown carbon fiber (VGCF) is used as shown in the work of Ting and Lake (1995). Recently, Naito et al. (2010) showed that grafting of high thermal conductivity carbon nanotubes (CNTs) is very effective in improving the thermal conductivity of certain types of carbon fibers, which can reach up to $47 \%$ improvement.

Overall, from the concise summary about passive mechanisms of enhancing heat transfer, it could be concluded that these mechanisms cannot be achieved without the presence of the enhancing elements. These elements are known as Heat Transfer Enhancers (HTE). Vortex generator (VG) is a type of HTE applied in CHE and researched by the scientific community. The Section 1.3 shows a brief history about VG application by industry and the main shapes found in open literature. In chapter 2 is presented a literature review on enhancement heat transfer by VG in compact heat exchanger.

\subsection{Vortex generators}

Firstly, vortex generators (VGs) have been used successfully in many aeronautical applications for reduction of separation in both internal and external flows. The vortices created transfer low energy fluid from the surface into the mainstream and bring higher energy fluid from the mainstream down to the surface, where the higher kinetic energy level is able to withstand a greater pressure rise before separation occurs. Hoerner (1985) provides a discussion and review of their use for lifting surfaces, such as, wings and control surfaces. Figure 3 shows a typical commercial transport aircraft with VGs built-on airplane wing. Especially for aircraft, controlling flow separation could result in an increase in system performance, with consequent energy conservation, as well as, weight and space savings. 


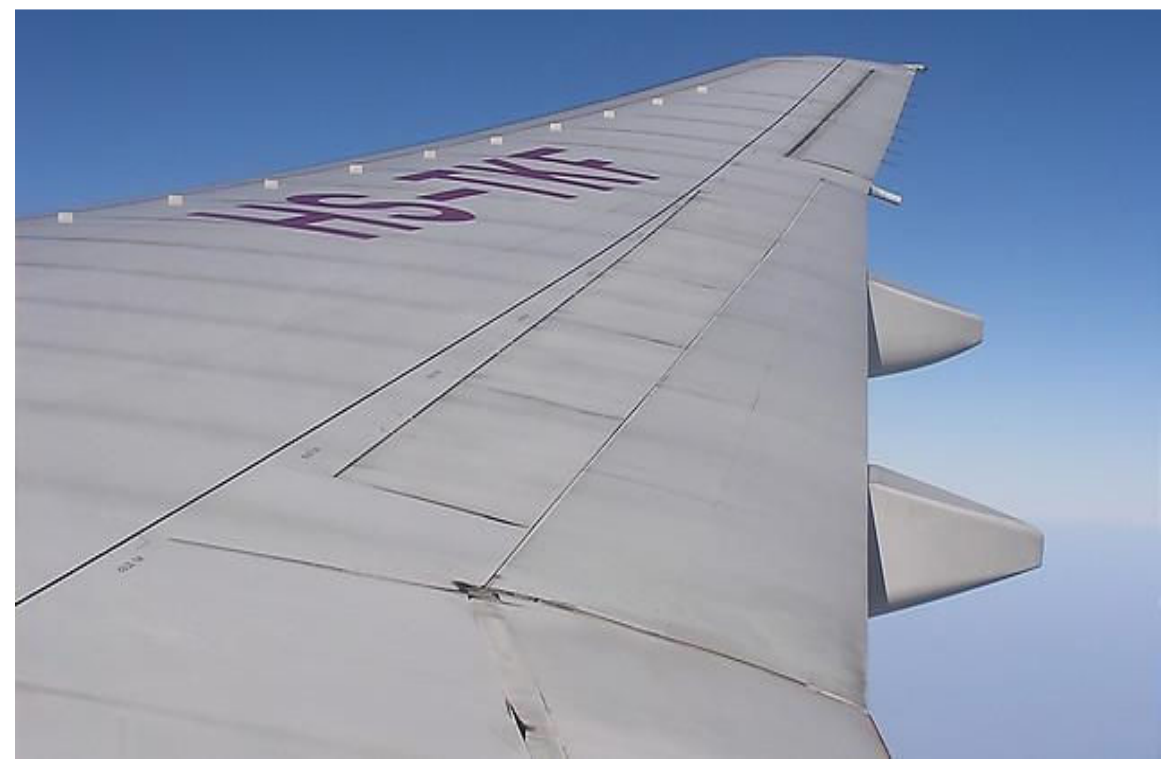

Figure 3 - Commercial transport aircraft with VGs.

Regarding to industrial applications, VGs are beneficial to transfer high momentum fluid from the outer flow into the boundary layer in order to increase the fluid temperature close to wall in a HE or to increase momentum to a boundary layer exposed to an adverse pressure gradient, in order to delay separation. In fact, both effects are probably occurring.

The first results about the influence of LVG on heat transfer were reported by Johnson and Joubert (1969). They investigated the influence of a row of triangular winglets on the drag and heat transfer of a circular cylinder normal to an air stream and found that the cylinder drag was reduced due to the separation delay caused by the longitudinal vortices and that heat transfer was somewhat increased. Edwards and Alker (1974) reported local heat transfer results for a row of co-rotating and counter-rotating vortices generated by a row of delta winglets attached to one channel wall. For the generation of co-rotating vortices the winglets had all the same attack angle, while for the counter-rotating vortices the attack angle alternated for successive winglets. Heat transfer and pressure drop data were first measured by Russell et al. (1982) for fin-tube geometry with longitudinal vortex generators in the form of very slender rectangular winglets. They state that triangular configurations and small attack angles gave disappointing performances in preliminary investigations. 
Delta and rectangular wing, delta and rectangular winglet, trapezoidal delta wing and ribs are some types of VGs. A great deal of research use these simple devices, due to its efficient, low maintenance and operating cost in HE. Figure 4 shows the most common VGs applied on CHE, as can be seen in literature review in Chapter 2.

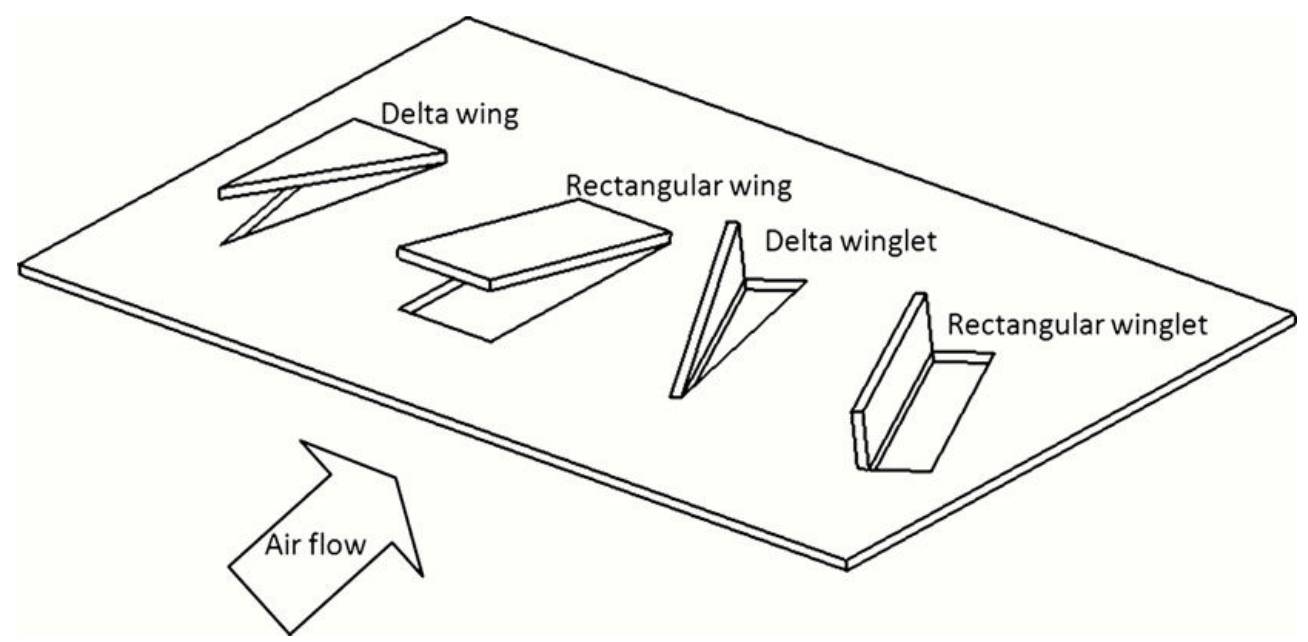

Figure 4 - Most common type of LVG.

There are two types of VGs: transverse vortex generator (TVG) and longitudinal vortex generator (LVG). The rotational direction of the TVG is normal to the main flow direction and the flow is 2-dimensional, while the LVG have their rotating axes parallel to the main flow and the flow is 3-dimensional. Figure 5 shows vortical structure by TVG, and Figure 6 shows by LVG. In Figure 6, Yanagihara and Torii (1991) describe three types of longitudinal vortices generated by a delta-winglet:

- Main vortex: it is formed due to flow separating at the tip of the delta-winglet and rolling up due to the lower pressure behind of the vortex generator;

- $\quad$ Corner vortex: it is generated at the corner between the front side of the deltawinglet and the fin;

- Induced vortex: it is created between the main and corner vortices. 
These vortices structures are generated by VGs, that are usually included in the flow on a plate or channel at an attack angle by either protruding wings or winglets or by punching holes on the surface. It is also known that is impossible to produce pure longitudinal vortices, since transverse vortices are always generated by the vortex generators. The attack angle determines the dominance of one over the other. It is normal thinks that when the attack angle is finitely small, mainly longitudinal vortices are generated and, as higher the attack angle, the more transverse vortices are generated. Maniar (2012) asserts that only transverse vortices are generated when the attack angle is $90^{\circ}$. In addition to these vortex structures shown in Figure 5 and Figure 6 , there is a natural vortical structure in the flow known as horseshoe vortex, illustrated in Figure 7. This vortex is also known as a longitudinal vortex. Consequently, the vortex structure generated by synergy between LVG and tube in a CHE is shown in Figure 8.

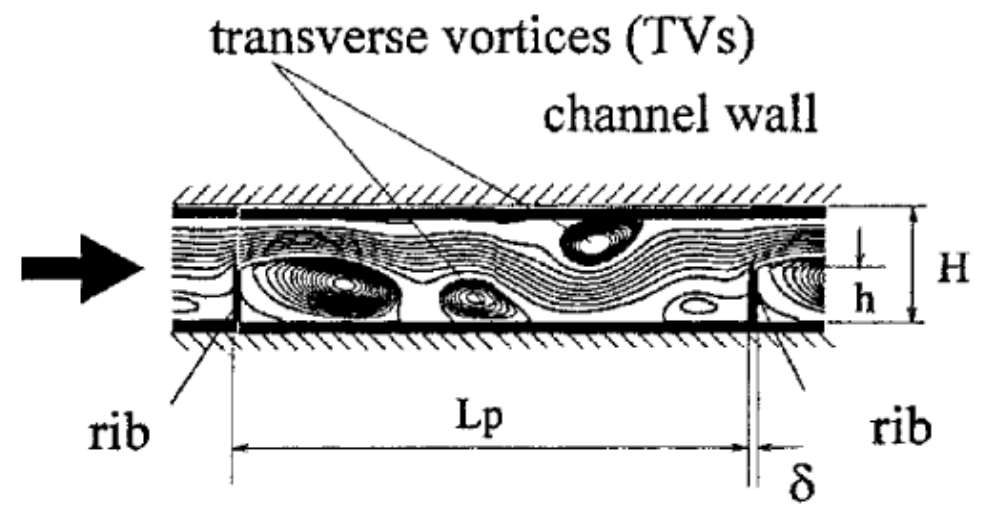

Figure 5 - Transverse vortex behind ribs in a channel (FIEBIG, 1998).

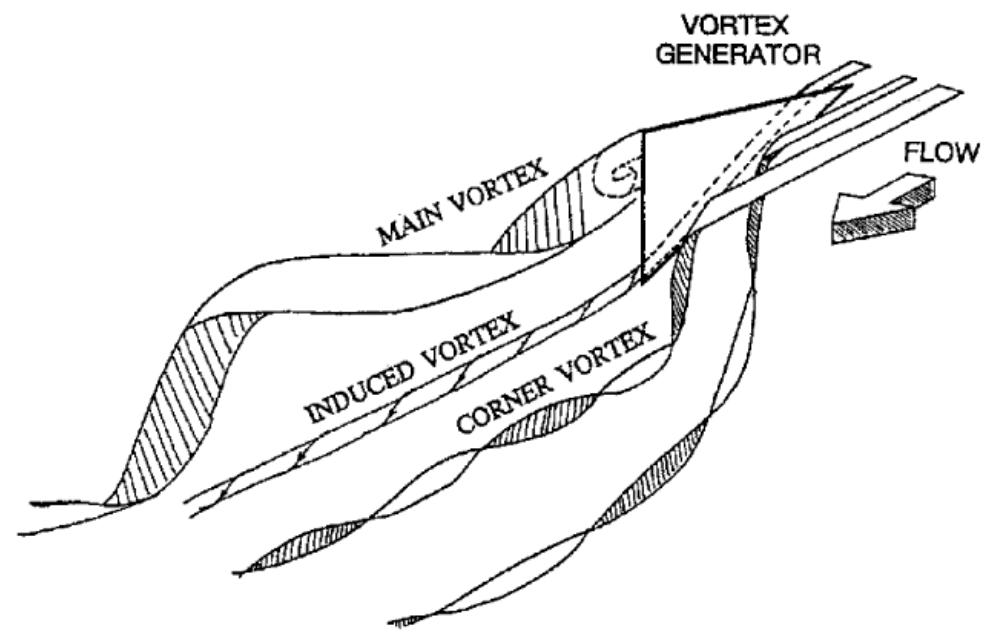

Figure 6 - Sketch of vortical structures generated by delta-winglet (YANAGIHARA AND TORII, 1991). 


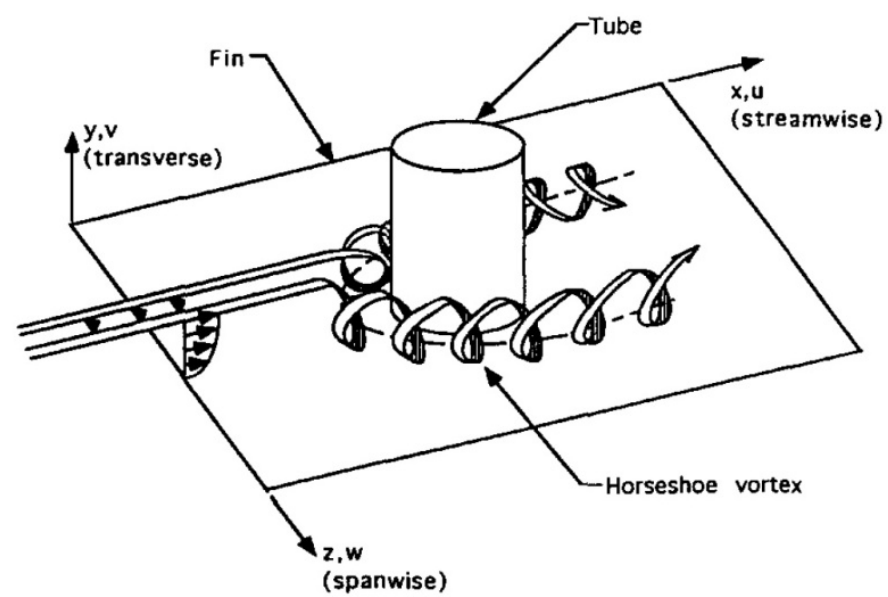

Figure 7 - The natural formation of a laminar horseshoe vortex (JACOBI and SHAH, 1995).

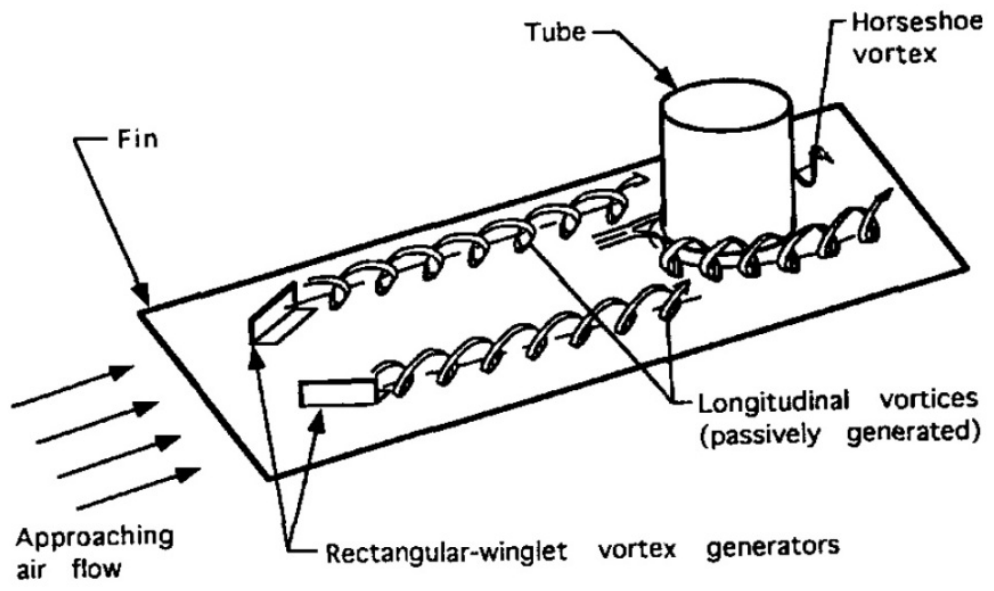

Figure 8 - Typical passively generated longitudinal vortices in CHE (JACOBI and SHAH, 1995).

These imposed vortical motions outlined, provide heat transfer performance enhanced with relatively low pressure penalty. This is due to pressure drop associated with wall friction and related to the derivative of the streamwise velocity (the spanwise and normal velocities have little effect); however, the spanwise and normal velocities play a significant direct role in convective heat transfer. The level of enhancement heat transfer and pressure drop depends on the VG geometry (size, shape, attack angle, aspect ratio, etc.) and the Reynolds number, Webb (2005). The effects of VGs on enhancement heat transfer in fin-tube CHE is discussed in Chapter 2. An appetizer is anticipated considering the conclusions from Fiebig (1995):

- VG generates appreciable augmentation heat transfer (on the average better than $30 \%$ ) over an area several hundred times the VG area; 
- The two most important parameters that influence heat transfer and pressure drop characteristics are the attack angle and VG shape. Delta forms are more effective than rectangular forms;

- Winglets provide higher heat transfer and pressure drop than wings (configurations illustrated in Figure 4);

- Heat transfer increases with attack angle up to maximum angle, which depends on the form of VG and Reynolds number;

- Pressure drop increases due to form drag of the VG and with attack angle.

Although the most researches consider the VGs shown in Figure 4, some researchers suggest unusual VG shape. Li et al. (2011) studied the VG shown in Figure 9. They claim that this VG shape is able to generate longitudinal vortices like traditional VG shown in Figure 4. The heat transfer performance and pressure drop is similar to rectangular winglet longitudinal vortex generators. For Reynolds number from 250 to 2500 (calculated at minimum cross-section of the flow area), the $\mathrm{JF}^{\frac{1}{3}}$ factor (detail about this performance criteria is discussed in Chapter 3 ) was $4.2 \%$ larger than heat exchanger with rectangular winglet longitudinal vortex generators. This outcome is quite small considering that delta VG is better than rectangular VG, Fiebig (1995 and 1998). Therefore, this result do not justify the complex shape that can be more difficult to manufacture (stamp).

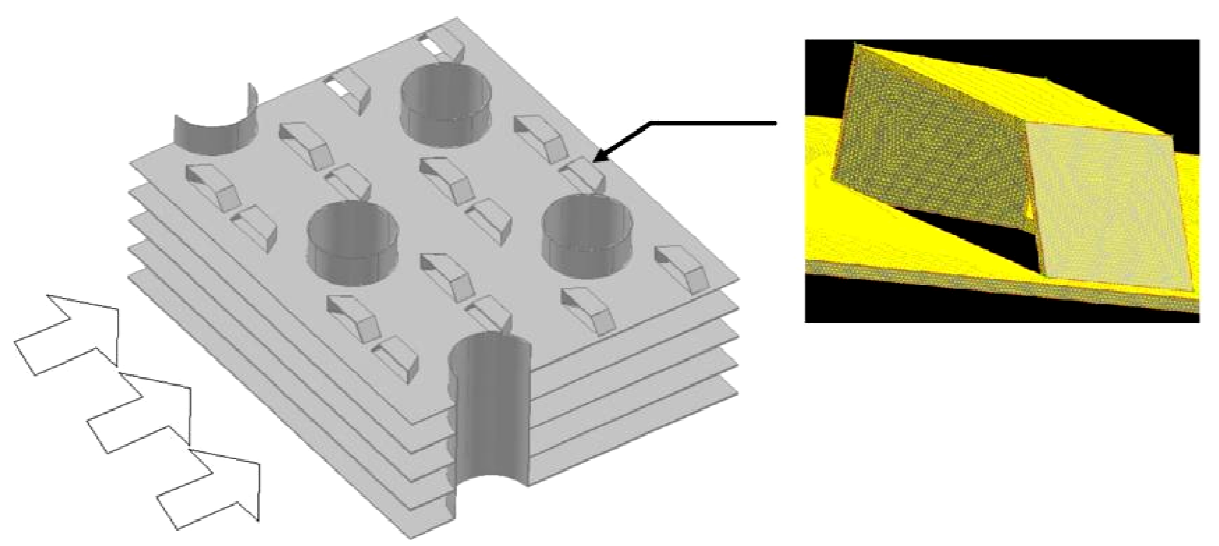

Figure 9 - Schematic diagram of core region of a slit fin-and-tube heat exchanger with longitudinal vortex generators (Li et al., 2011) 
Wang et al. (2002) proposed a different type of VG shown in Figure 10, considering inline fin-tube arrangement. This VG is known as wave-type VG. The main feature of this VG is that it is able to produce horseshoe vortical structure like those that appears around the tube. As it is very well known, between the first and second tube there is a recirculation region where the heat transfer coefficient is very low. The authors claim that this recirculation phenomenon is almost disappeared with the presence of their vortex generators. The presence of vortex generators significantly increases the vortical motions of the horseshoe vortices hitting on the tubes. Also, a much better mixing characteristic is noted for introducing the vortex generators. The friction penalty was about $25-55 \%$ higher than that of the fin-plain geometry. Results about heat transfer was not reported. The optimization process adopted in this thesis, considering the input parameters range, is also able to evaluate the VG impact when it is fixed in front of the tubes. Thus, if this feature mentioned by Wang et al. (2002) were important, the optimization process can cover this.

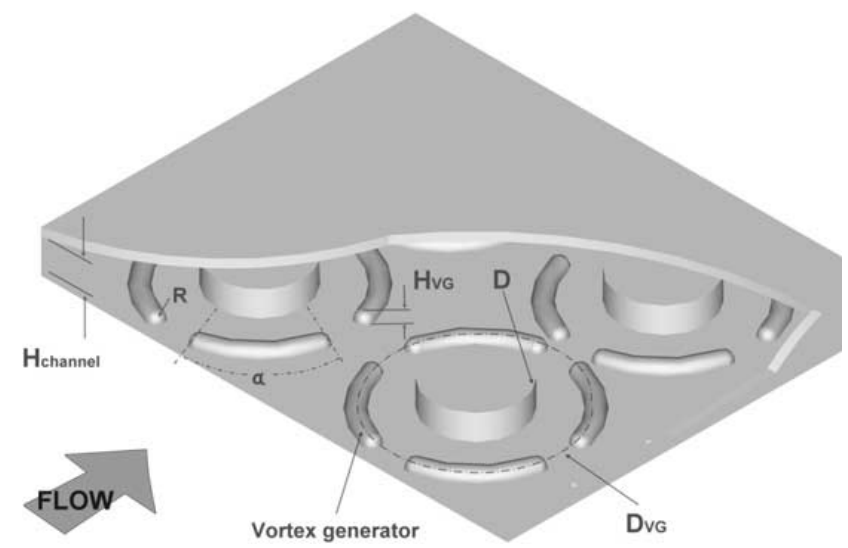

(a)

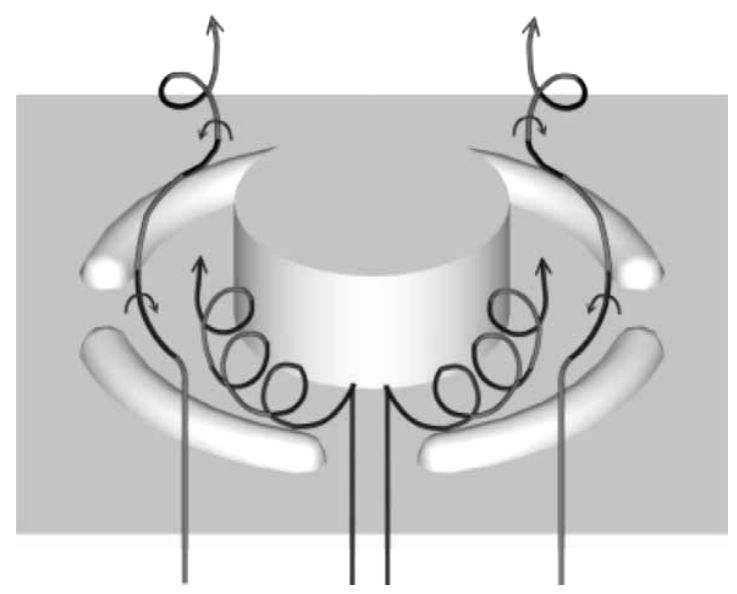

(b)

Figure 10 - (a) Configuration of the VG geometric proposed, (b) Schematic of the flow motions across the vortex generators, Wang et al. (2002).

Zhou and Ye (2012) experimentally investigated and compared the performance of a pair of VG: rectangular winglet, trapezoidal winglet, delta winglet and curved trapezoidal winglet, as shown in Figure 11. Obviously, only trapezoidal winglet and curved trapezoidal winglet are an unusual VGs. The Reynolds number range was very large from 700 to 26,800 , covering laminar, transitional and turbulent region. Their conclusions showed that delta winglet was the best device in laminar and 
transitional flow region, while curved trapezoidal winglet had the best JF factor in fully turbulent region. In most of tested configurations by researchers, the JF factor was higher than 1, when Reynolds number was higher than 7,000. When JF factor is higher than 1 , the heat transfer is higher than the drop pressure penalty.

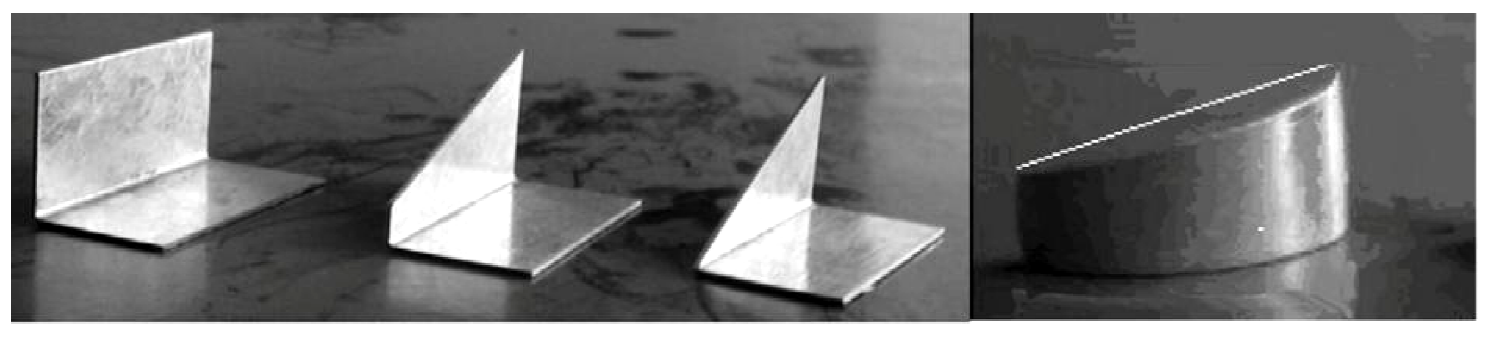

Rectangular winglet Trapezoidal winglet Delta winglet $\quad$ Curved trapezoidal winglet

Figure 11 - Pictorial diagram of vortex generators, Zhou and Ye (2012).

Besides of these unusual vortex generators previously shown, He et al. (2012) also studied the potential of punched winglet type vortex generator arrays. The arrays are composed of two delta-winglet pairs with two layout modes of continuous and discontinuous winglets, Figure 12. The heat transfer performance of two array arrangements were compared to a conventional large winglet configuration for the Reynolds number ranging from 600 to 2600, based on the outside tube diameter. The authors reached interest conclusions. For the punched VGs, the corner vortex, (Figure 6), showed the characteristics of main vortex and plays a more important role on the fluid flow behavior and heat transfer characteristic in the channel of fin-tube heat exchanger than that of the main vortex. The VG arrays generated more vortices and influenced each other, which weaken the swirling movement of the fluid, especially for the continuous small winglet array. In addition, the vortices generated by large winglets can affect the whole region of fin channel height, while the VG arrays can only influence the fluid flow structure of local fin channel. For side arrangements of VGs, the discontinuous small delta winglets case showed the best heat transfer enhancement, and a significant augmentation up to $33.8-70.6 \%$ in heat transfer coefficient was achieved accompanied of pressure drop penalty of 43.4$97.2 \%$. Meanwhile, the large winglets case represented the lowest pressure drop and highest value of JF factor. For VGs arrangements with three different values of attack 
angles $\left(10^{\circ}, 20^{\circ}\right.$ and $\left.30^{\circ}\right)$, the discontinuous small winglet array always showed the best heat transfer improvement and also largest pressure loss. This alternative study was really interesting and the results can be summarized as the large winglet keeps being the good option when the JF factor is the target. If the pressure drop penalty is not a concern, the discontinuous small delta winglets case achieved the best ratio of heat transfer.

Especially regarding these last results by $\mathrm{He}$ et al. (2012), the singular delta winglet is still the preferred choice for the best JF factor and this strengthens the choice done in this present work, considering the VG shape discussed in Section 1.4.

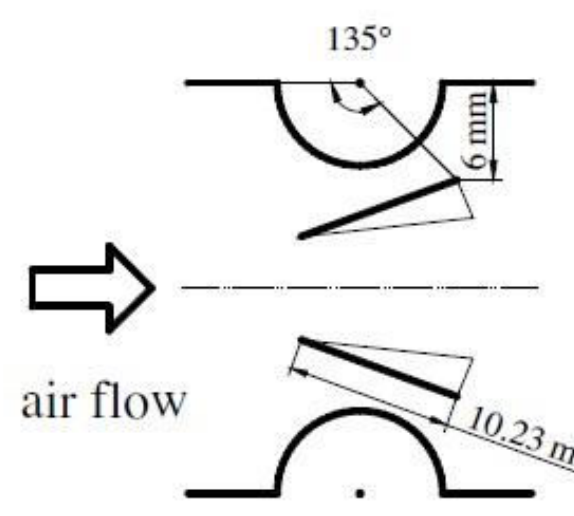

Large winglet

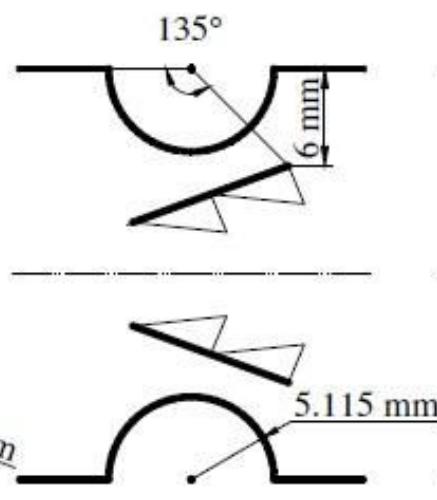

Array-A

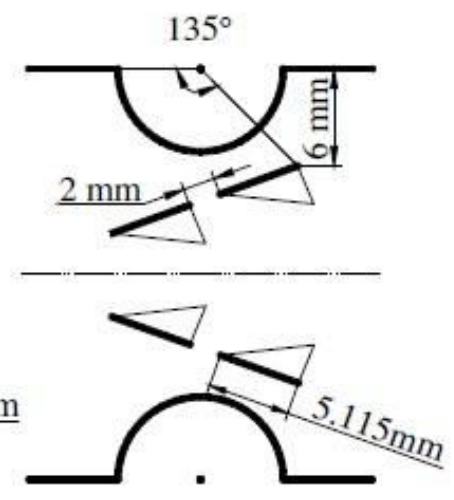

Array-B

Figure 12 - Schematic of the large winglet and two kinds of delta winglet arrays geometry and placements, He et al. (2012).

\subsection{Objective of this thesis}

As mentioned earlier in this chapter, saving thermal energy, reduction of material and cost reduction are key words nowadays regarding heat transfer optimization. According to this trend, the present study is focused on enhance of the heat transfer considering a fin-tube CHE with two VGs and two row tubes, as shown in Figure 13. Based on Colburn factor (j) and Friction factor (f), four objective functions are evaluated, which are presented in Chapter 4, according to thermal-hydraulic dimensionless presented in Chapter 3. 
A typical industrial application was chosen and the common dimensions of a automotive CHE were defined. The Reynolds number was calculated considering specific validation tests, eg, the inlet velocity at CHE face covering both lower and higher velocities. Reynolds number was not a variable parameter for each metamodeling and optimization process. Thus, this was set as $\operatorname{Re}=250$ and $\operatorname{Re}=1400$, and two similar procedures were conducted for each Reynolds number. Reynolds number is based on fin pitch, according to Torii et al. (2002), which allows comparison with the most relevant papers in open literature. However, for numerical validation, the Reynolds number is based on fin collar outside diameter $\left(\operatorname{Re}_{c o}\right)$, according to Wang et al. (2000). The main goals of the work could be summarized as following:

1. Current publications consider, symmetrically, the position-x, position-y and rotate- $\theta$, for both VGs. In the present work, the VGs parameters are considered independently, eg, for each VG are attributed four input data: position-x, position-y, rotate- $\theta$ and rotate- $\varphi$. As can be seen in Chapter 2, rotate- $\varphi$ was not evaluated by current papers;

2. In current researches, the interaction among VG parameters is not reported. Herein, the interaction among the eight parameters and the level of variance contribution of each parameter in augmentation heat transfer were evaluated;

3. There is not consensual among researchers about the optimum parameters set to increase the augmentation heat transfer for different Reynolds number. Herein, is reported the optimal VG parameters for each Reynolds number evaluated;

4. Facing the results from two approaches applied to optimization procedure through Response Surface Methodology (RSM) and Direct Optimization (DO).

In Figure 13, the yellow points show the coordinates in direction $\mathrm{x}$ and $\mathrm{y}$ on the bottom fin and the rotation center around the axis-z $(\theta)$. These yellow points are placed exactly at half chord of the vortex generator for both VGs. Reference position is shown in Figure 14. 


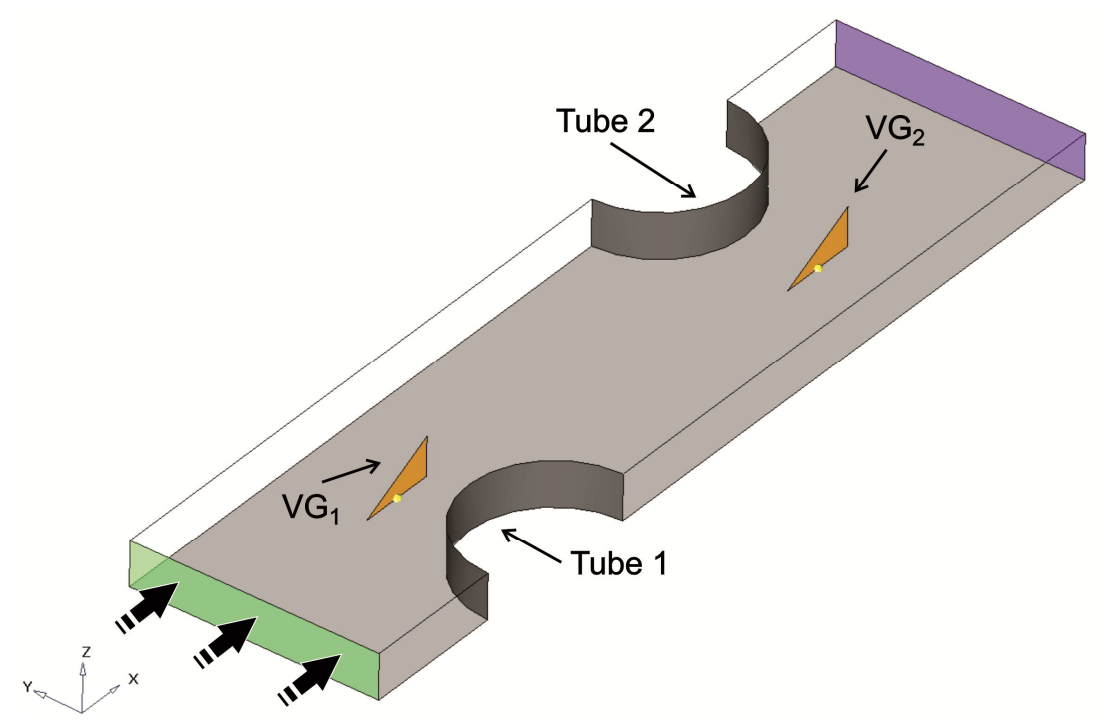

Figure 13 - The main domain of the CHE with two tubes and two delta winglet VGs.

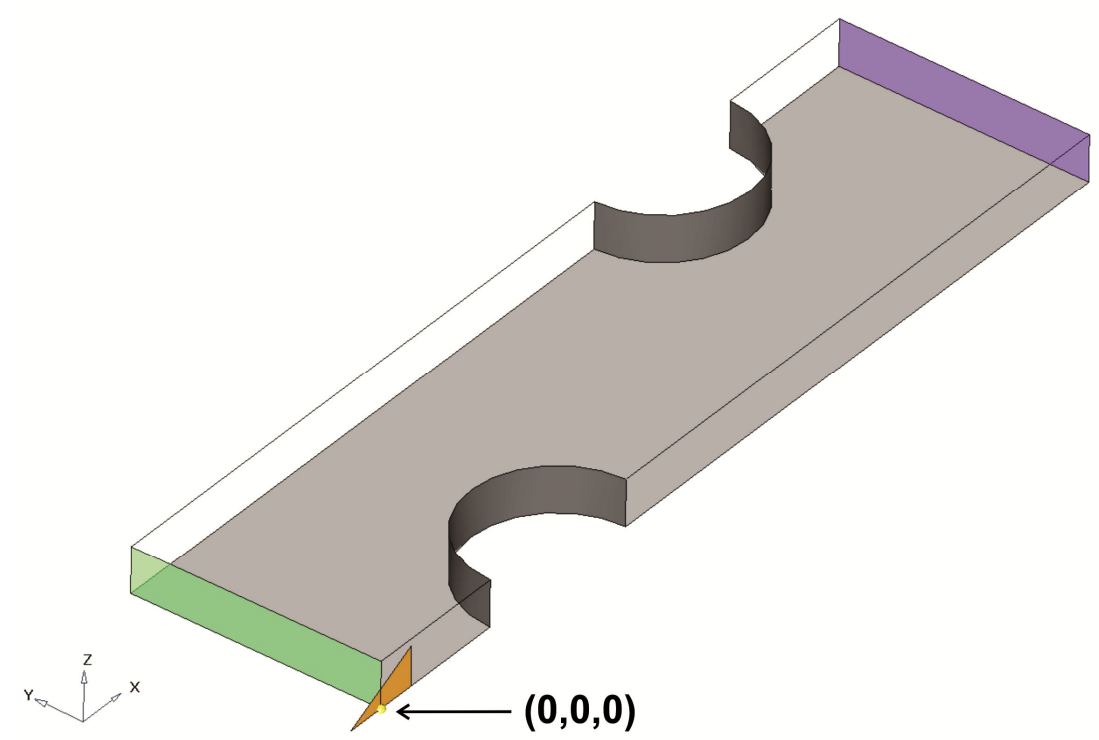

Figure 14 - The reference position of the VG on the main domain.

\subsubsection{Parameters}

As mentioned in Section 1.4, eight independent parameters were considered in present work, as following: 


\section{a. VGs position:}

Each vortex generator attached on the bottom fin, moves in the $x$ and $y$ directions, Figure 15. The range was established to avoid collision between VGs. This was made by restricting the VGs displacement in the direction-x. Also, the restriction for the axis-y was made to avoid the interference between VGs and symmetries. The interferences between VGs and tubes were guaranteed considering the constraint as following:

$$
\begin{aligned}
& \left(x_{1}-x_{t 1}\right)^{2}+\left(y_{1}-y_{t 1}\right)^{2} \geq f\left(r_{t 1}^{2}\right)=40 \\
& \left(x_{2}-x_{t 2}\right)^{2}+\left(y_{2}-y_{t 2}\right)^{2} \geq f\left(r_{t 2}^{2}\right)=40
\end{aligned}
$$

where:

$\mathrm{x}_{\mathrm{t} 1} \rightarrow$ Center point of the tube 1 at axis-x.

$\mathrm{x}_{\mathrm{t} 2} \rightarrow$ Center point of the tube 2 at axis- $\mathrm{x}$.

$y_{t 1} \rightarrow$ Center point of the tube 1 at axis-y.

$\mathrm{y}_{\mathrm{t} 2} \rightarrow$ Center point of the tube 2 at axis-y.

$r_{t 1} \rightarrow$ Radius of the tube 1.

$r_{\mathrm{t} 2} \rightarrow$ Radius of the tube 2 .

$f\left(r_{t 1}^{2}\right) \rightarrow$ Constant as function of the radius of the tube 1.

$f\left(r_{t 2}^{2}\right) \rightarrow$ Constant as function of the radius of the tube 2 .

The constant value of 40 shown at right side in Equations 1 and 2, is calculated as function of the radius of the tubes, beyond the both VGs angles. This value is slightly higher than $r_{t}^{2}$ due to the VGs angles and position are close to boundary domain. 
These constraints were used during DOE (Design of Experiment) step to delete the settings outside of the main domain.

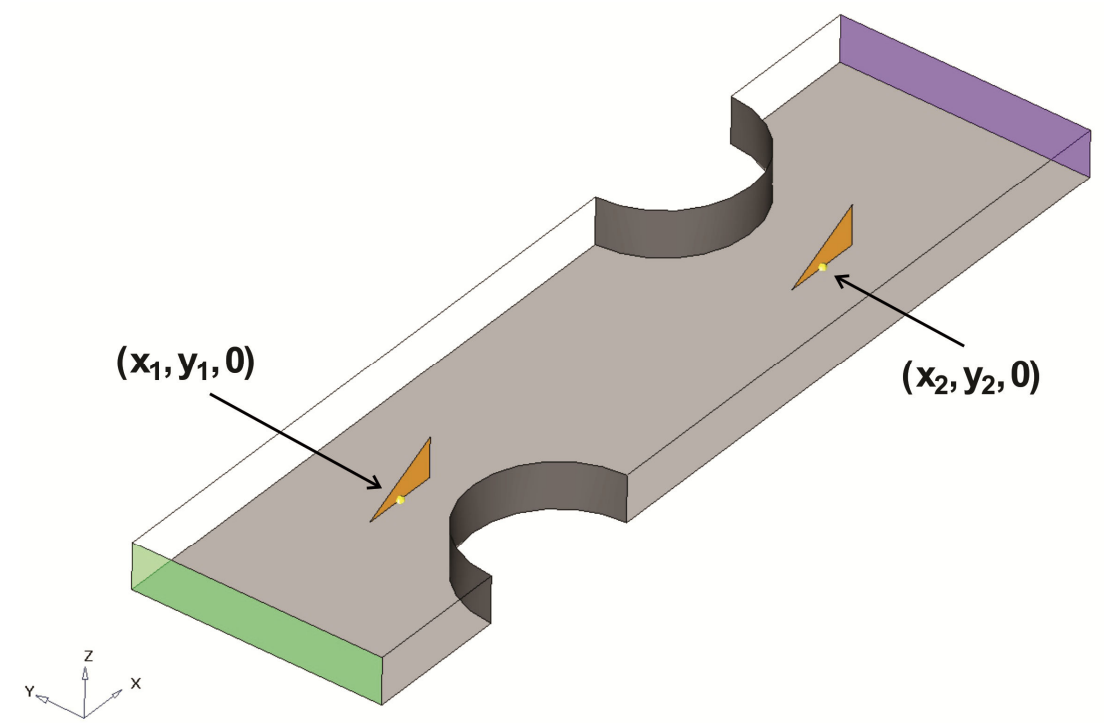

Figure 15 - VGS position attached to the bottom fin.

\section{b. VGs angles:}

Each vortex generator attached on the bottom fin could rotate around the axis$x(\varphi)$ and axis-z $(\theta)$, as shown in Figure 16 and Figure 17, respectively. As mentioned in the abstract, the angle $\varphi$ is called by rolling angle. As it can be seen in Table 2, the angle $\varphi$ was limited because the grid quality concerns between bottom fin and VGs, eg, if $\varphi$ is very small, for example $\varphi=10^{\circ}$, for a good grid quality (residual skewness less than 0.5 and aspect ratio less than 2.0), it would be necessary a mesh too refined close to the walls, consuming a high computational resource to solve the governing equations. The first assessments about the RSM (Response Surface Methodology) outputs revealed that a big set would be necessary for reached a satisfactory RSM and, thus, a case that running in a long time is not desired. This assessment is confirmed and discussed in Chapter 4 and Appendix B. 


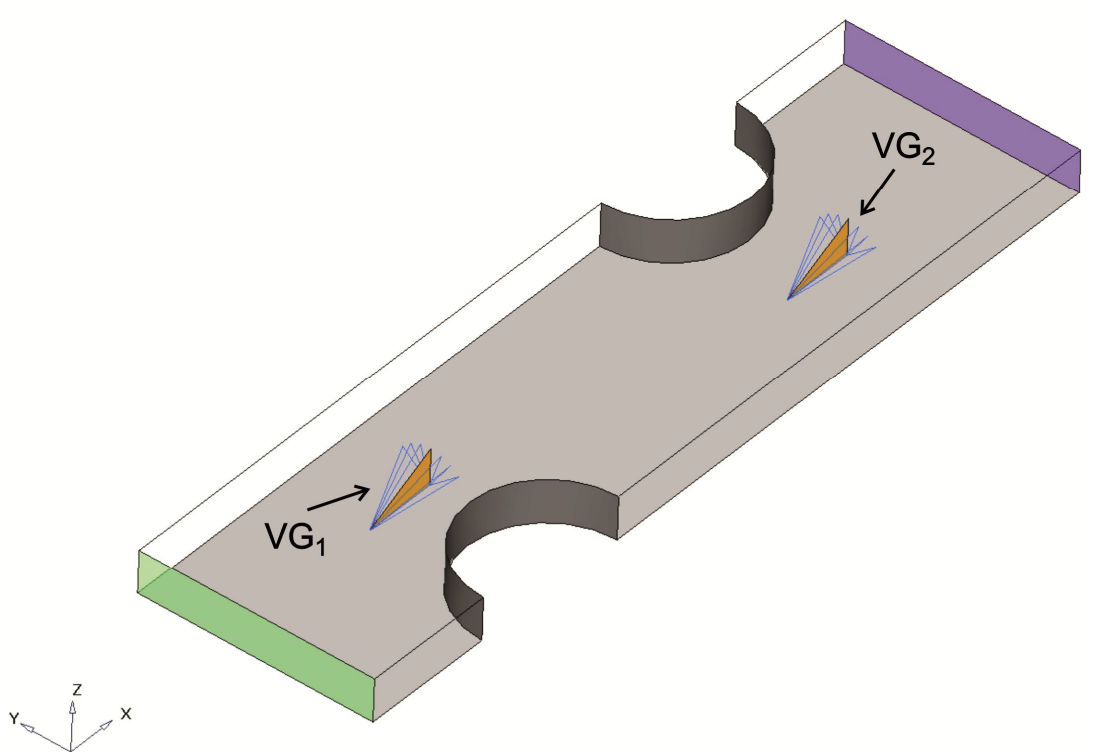

(a)

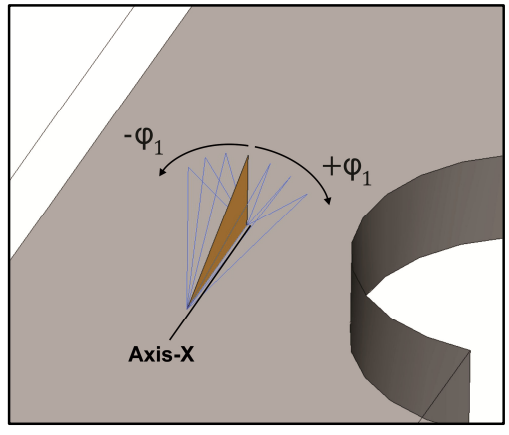

(b)

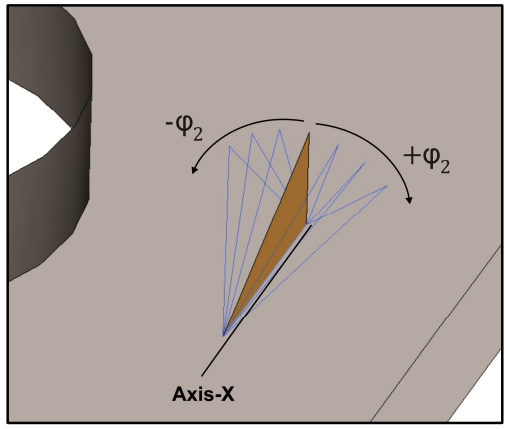

(c)

Figure 16 - (a) Overview of the main domain, Axis-x, (b) Highlight of the $V G_{1}$, (c) Highlight of the $V_{G_{2}}$.

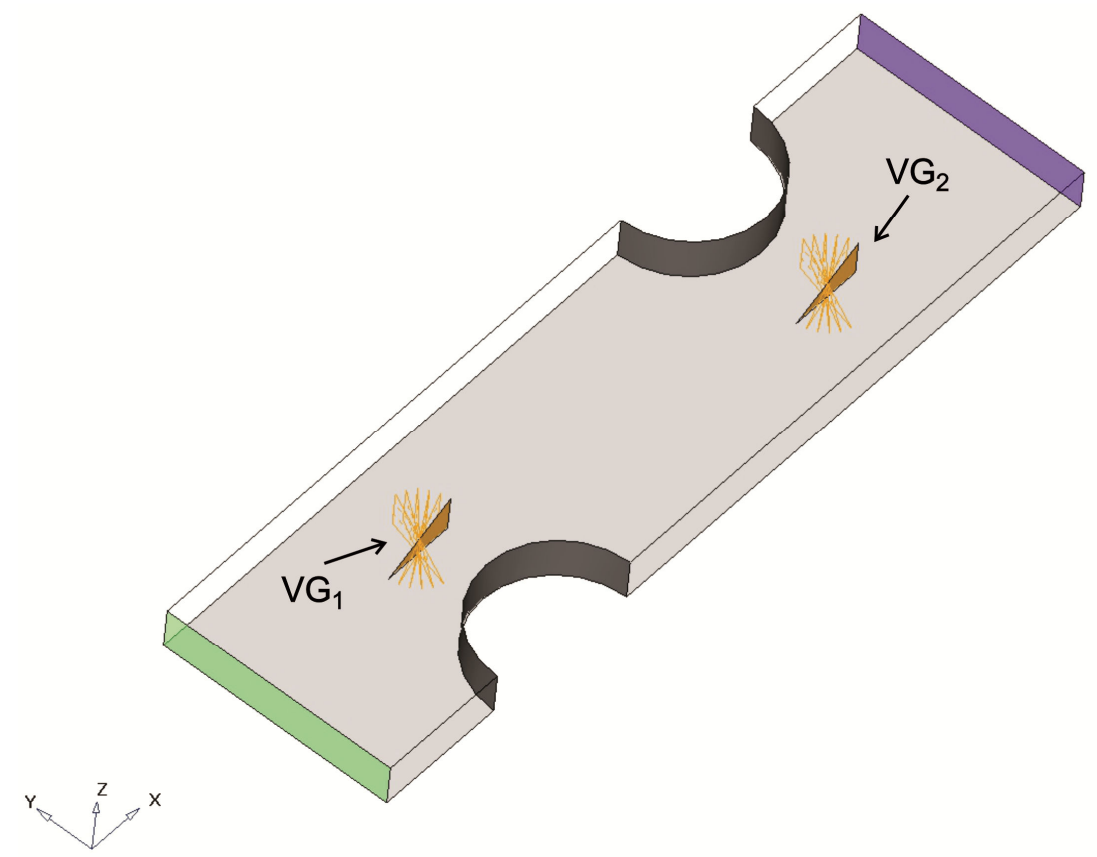

(a) 


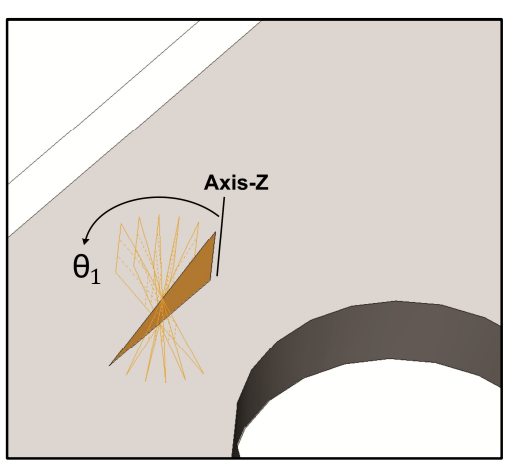

(b)

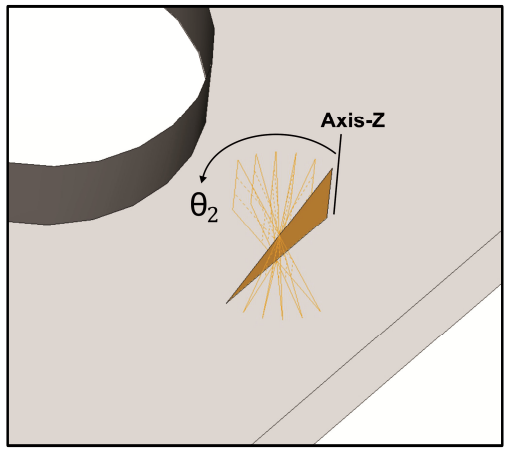

(c)

Figure 17 - (a) Overview of the main domain. Axis-z, (b) Highlight of the $V G_{1}$, (c) Highlight of the $V G_{2}$.

Overall, the eight independent parameters evaluated in this work were shown, as well as the main concerns regarding the interference between VGs and boundaries (symmetries and tubes). These concerns outlined guided the parameter ranges set for each input parameter, which is shown in Table 2.

Nevertheless, Chapter 3 shows in details the full domain dimensions chosen for this work (fin pitch, transversal and longitudinal tube pitch, fin thickness and depth), considering a typical automotive application, beyond the model description and numerical method and thermal-hydraulic dimensionless parameters. Numerical validation procedure is presented in Appendix A. Chapter 4 presents the optimization statement of the problem outlined and the constraints for both approaches: optimization by surrogate model (RSM) and direct optimization (DO). RSM quality is discussed in Appendix $B$ and a quick comparison among surrogate model based on Neural Networking, Radial Basis Function (RBF) and Kriging is also presented. Chapter 5 presents the results and discussion for both $\operatorname{Re}=250$ and $\operatorname{Re}=1400$. Sensibility analysis was conducted (main and interaction effects) for the Colburn and Friction factors, which the main differences between them are also compared. Pattern study focused on vortices structures produced by VG configuration was also made. Chapter 6 presents the main conclusions and some recommendations for future works. 
Table 2 - Parameters ranges applied to meta-modeling and optimization.

\begin{tabular}{ccc}
\hline Parameter & Range & Constraint \\
\hline $\mathrm{x}_{1}(\mathrm{~mm})$ & $2.5 \leq \mathrm{x}_{1} \leq 17.5$ & Equation 1 \\
$\mathrm{y}_{1}(\mathrm{~mm})$ & $2.5 \leq \mathrm{y}_{1} \leq 8.5$ & - \\
$\theta_{1}\left({ }^{\circ}\right)$ & $0 \leq \theta_{1} \leq 359$ & - \\
$\varphi_{1}\left({ }^{\circ}\right)$ & $-50 \leq \varphi_{1} \leq+50$ & \\
$\mathrm{x}_{2}(\mathrm{~mm})$ & $22.5 \leq \mathrm{x}_{2} \leq 37.5$ & Equation 2 \\
$\mathrm{y}_{2}(\mathrm{~mm})$ & $2.5 \leq \mathrm{y}_{2} \leq 8.5$ & - \\
$\theta_{2}\left(^{\circ}\right)$ & $0 \leq \theta_{2} \leq 359$ & - \\
$\varphi_{2}\left(^{\circ}\right)$ & $-50 \leq \varphi_{2} \leq+50$ & - \\
\hline
\end{tabular}




\section{Literature review on heat transfer enhancement by longitudinal vortex generators (LVG)}

As fluid passes across the LVG, strong secondary swirling flow is generated, and the tangential velocity of the vortices can be as high as twice the main flow velocity. The high-velocity swirling secondary flow not only promotes mixing of the fluids in the main flow and in the edge regions, but also can guide the high-energy fluid into the boundary layer to suppress and/or delay boundary layer separation, with a corresponding decrease of profile drag. By appropriate arrangement of LVGs, heat transfer on the air side of the heat exchanger can be enhanced while the pressure drop is decreased in some cases, Kwak et al. (2005) and Torii et al. (2002). Although introduction of LVGs on the air side causes an increase of profile drag for the fins, properly designed LVGs can delay flow separation on the tube so that the profile drag for the tube is decreased.

The early studies of LVGs focused on their application to straight channels, where the flow structures are relatively simple. While most early studies were performed experimentally, subsequent research made use of numerical simulation for the application of LVGs to fin-and-tube heat exchangers. These numerical investigations were made possible due to the rapid development of computer hardware and software. The characteristics of fluid flow and heat transfer can now be thoroughly revealed by numerical simulation and high-level post-processing. 


\subsection{Heat transfer enhancement by LVGs in fin-and-tube HE}

According to He and Zhang (2012), the application of LVGs on fin-and-tube heat exchanger with two rows of tubes is not thoroughly investigated, although is widely used in the modern residential air conditional system. Lei et al. (2010) presented an study of hydrodynamics and heat transfer characteristics, easy to understand, of a novel heat exchanger with two rows of tubes and delta-winglet vortex generators. This study start this section because it shows a classical way applied to analysis when vortex generators are considered. As it can be seen in this review, the main parameters generally studied (aspect ratio and attack angle) are separately evaluated and the interactions between those are not often reported. In this present thesis, the interaction study among the parameters described in Chapter 1 was made. Thus, He and Zhang (2012), based on the work made by Lei et al. (2010), compiled the main results and conclusions, which are partially reported below. Figure 18 shows the schematic diagram of the computational domain of the fin-tube heat exchanger with two rows of tubes, while the sizes of the computational domain, position and dimensional of VGs are shown in Figure 19.

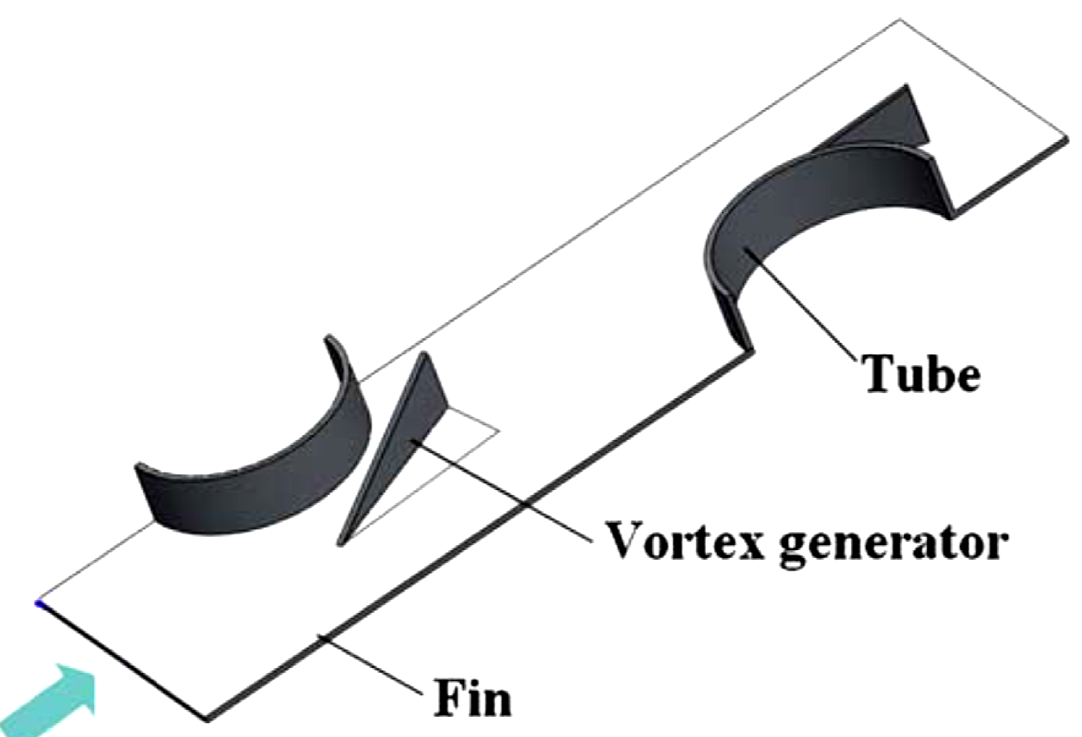

\section{Flow direction}

Figure 18 - Overview of the fin-and-tube heat exchanger, Lei et al. (2010). 

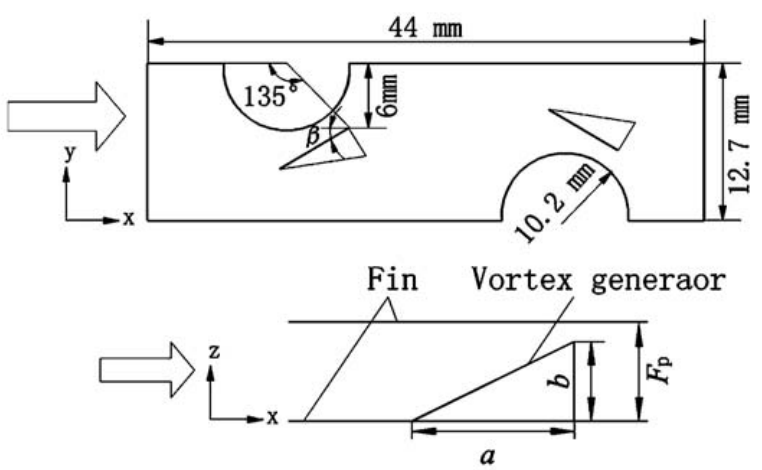

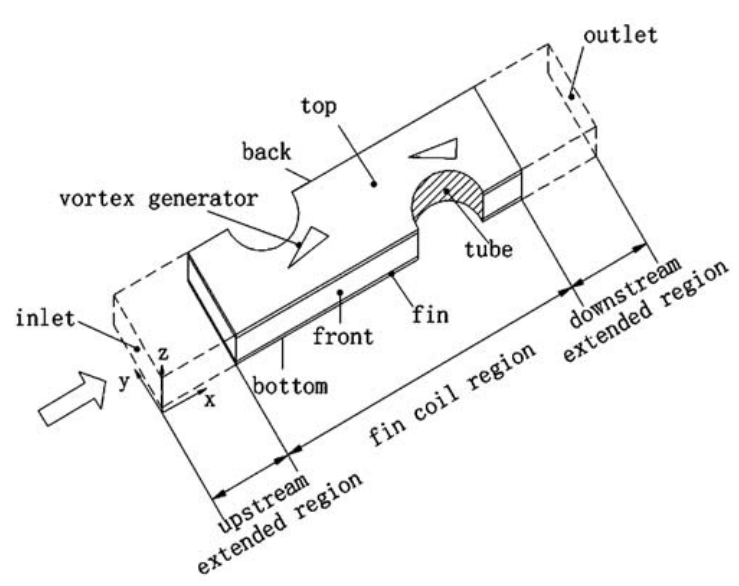

Figure 19 - Dimensions/position of the VGs and computational domain, Lei et al. (2010).

Aspect ratio of vortex generator was defined as $(\Lambda=a / b)$. The authors evaluated the aspect ratio 1, 2, 3 and 4 . The local flow field and temperature distribution in a finand-tube heat exchanger with side delta winglets were firstly analyzed. In Figure 20, a vortex whose axis is the same as the main flow direction is generated. When fluid flows across the delta winglet, the pressure difference across the delta winglet results in secondary flow and generates the vortex.

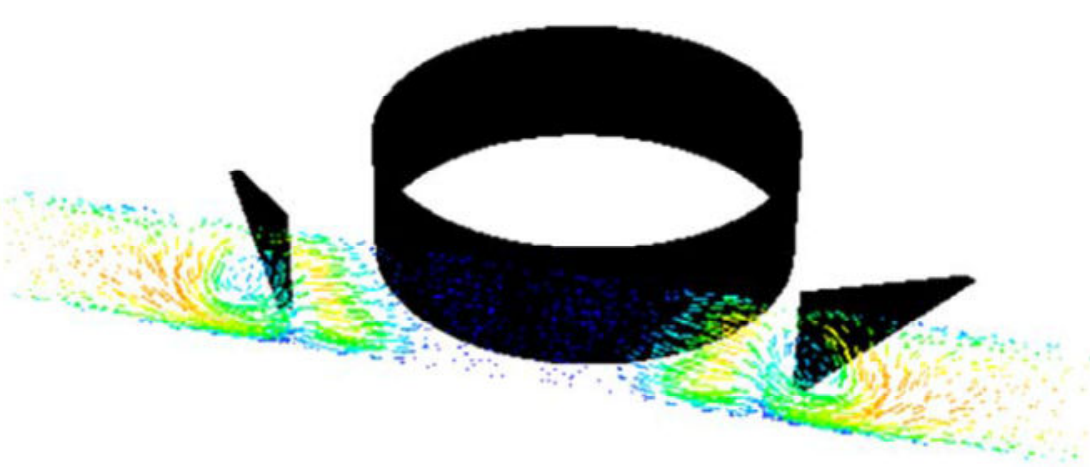

Figure 20 - Structure of longitudinal vortex $(\operatorname{Re}=1000)$ for aspect ratio 2, Lei et al. $(2010)$.

Vortex disturbs the fluid flow and reduces the boundary layer thickness. Figure 21 shows velocity vector plotted on a plane for both the enhanced and unenhanced configurations at $\operatorname{Re}=1000$ (based on fin collar outside diameter). The plane is close to the bottom fin surface at a distance of axis- $\mathrm{z}=0.3 \mathrm{~mm}$ from the bottom fin. In Figure 
21 , the recirculation occurs widely behind the tubes, which further deteriorates the heat transfer. When the vortex generator with common flow-up arrangement is employed, a nozzle-like passage appears between the tube and delta-winglet vortex generator. The fluid accelerates in the constricted passage and significantly delays the flow separation. Thus, the size of the wake zone and form-drag was significantly decreased. Consequently, the addition of the delta winglet not only generates a longitudinal vortex, but also forms a nozzle-like acceleration zone that decreased the size of the wake zone behind the tube.

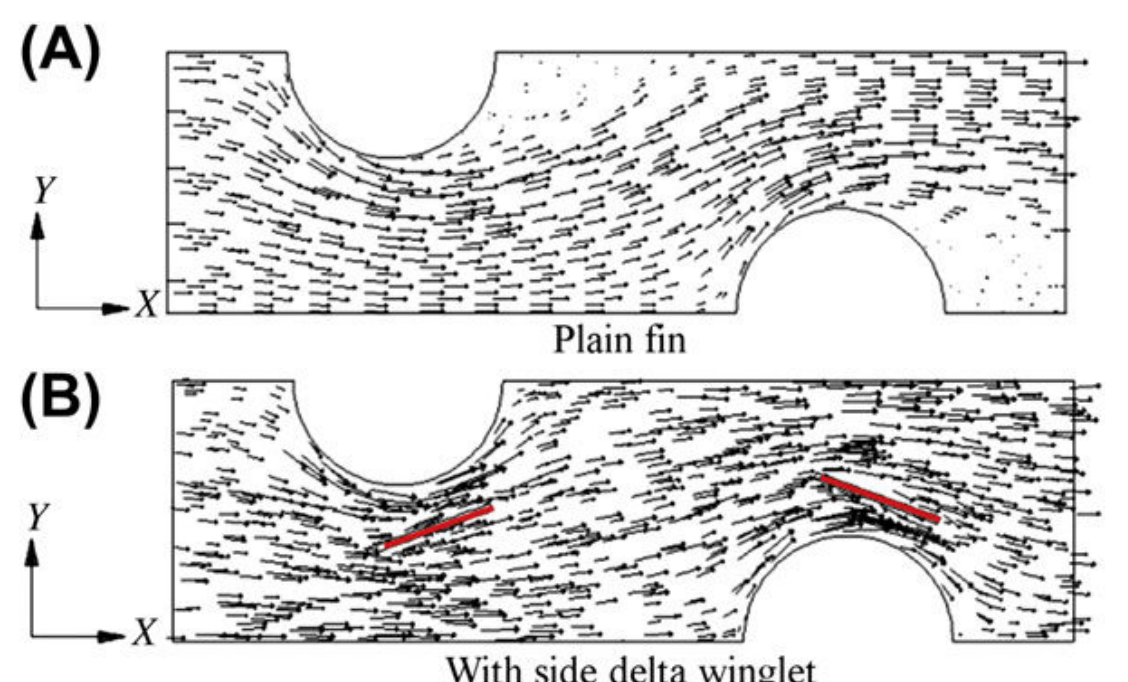

Figure 21 - Velocity vector plots on a plane near the fin $(R e=1000)$ for aspect ratio 2. (A) Plain fin. (B) With side delta winglet. Lei et al. (2010).

Figure 22 shows the exit temperature field for plain fin and fin with delta winglets for $\operatorname{Re}=1000$. Exit temperature for the case of plain fin was vertically symmetric with respect to the middle-plane and asymmetric for the case with vortex generator because the swirling flow rearranged the temperature distribution in the fluid. The air temperature gradient on the side with vortex generator was increased and the exit air temperature was higher than the case of plain fin. In other words, the difference between the air temperatures at inlet and outlet was increased. Consequently, the heat transfer rate with vortex generator was higher than the case of plain fin. 
a

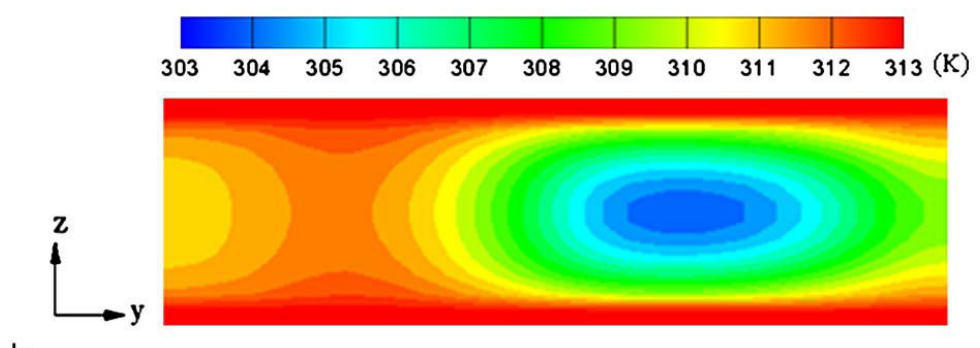

b

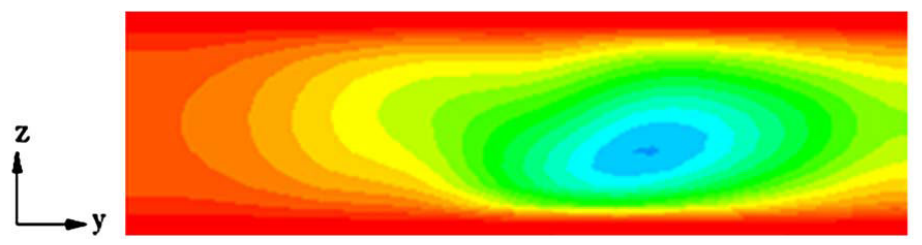

Figure 22 - Exit temperature field for aspect ratio 2. (A) Plain fin. (B) With VG. Lei et al. (2010).

Figure 23 shows the distribution of fin surface temperature for plain fin and fin with delta winglet for $\operatorname{Re}=1000$. Lei et al. (2010) considered fixed temperature on the tube, ie, the heat transfer by conduction process is resolved on fin. This was considered in this thesis only for numerical validation, because the long time of each case turns the optimization process impracticable considering the huge amount of cases needs for a good RSM (this is discussed in Chapter 4 and Appendix B), ie, fin and tube temperature was fixed and the heat transfer by conduction process was not resolved. Temperature gradient in the region behind the tube for the case with vortex generator was higher than the case of plain fin without vortex generator. At the same location, the local temperature with vortex generator was lower than for plain fin. The average fin temperature was also lower due to addition of vortex generators. Thus, the delta winglet increased the heat transfer rate and, as a consequence, increased the overall heat transfer coefficient so that the heat transfer performance was improved. The tubes in the fin-tube heat exchangers can be arranged either inline or staggered. 
(A)

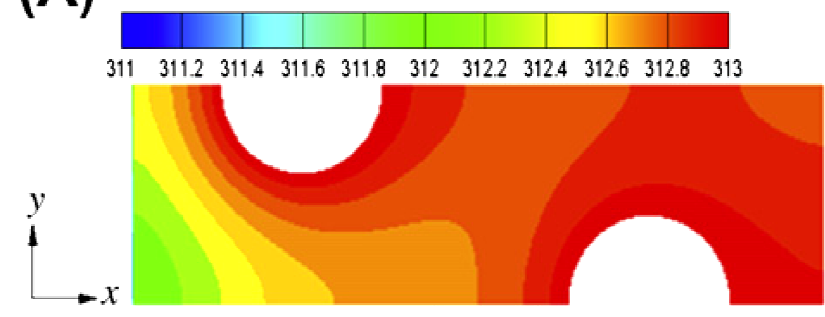

Plain fin

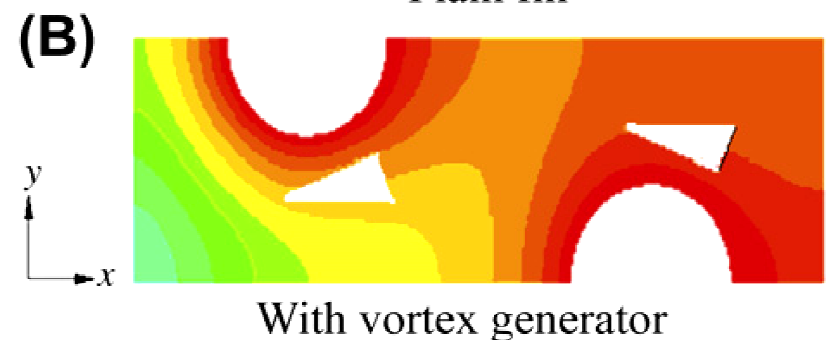

Figure 23 - Fin surface temperature field for aspect ratio 2. (A) Plain fin. (B) With VG. Lei et al. (2010).

Figure 24 shows the variation of Colburn factor $(j)$ as function of Reynolds number for different arrangements. For the same Reynolds number, the Colburn factor for the case with vortex generators was higher than that of the plain fin, regardless of the arrangements. The mechanisms of heat transfer enhancement can be explained by the fact that the delta winglet generated a secondary flow which allowed the fluid to directly impinge to the fin surface. The boundary layer became thinner and the hot and cold fluids were more vigorously mixed. In addition, there exists an accelerated zone between the side delta winglet, tube wall, and fin, which allowed the fluid to enter the poorly region behind the tube and reduced the size of the wake zone.

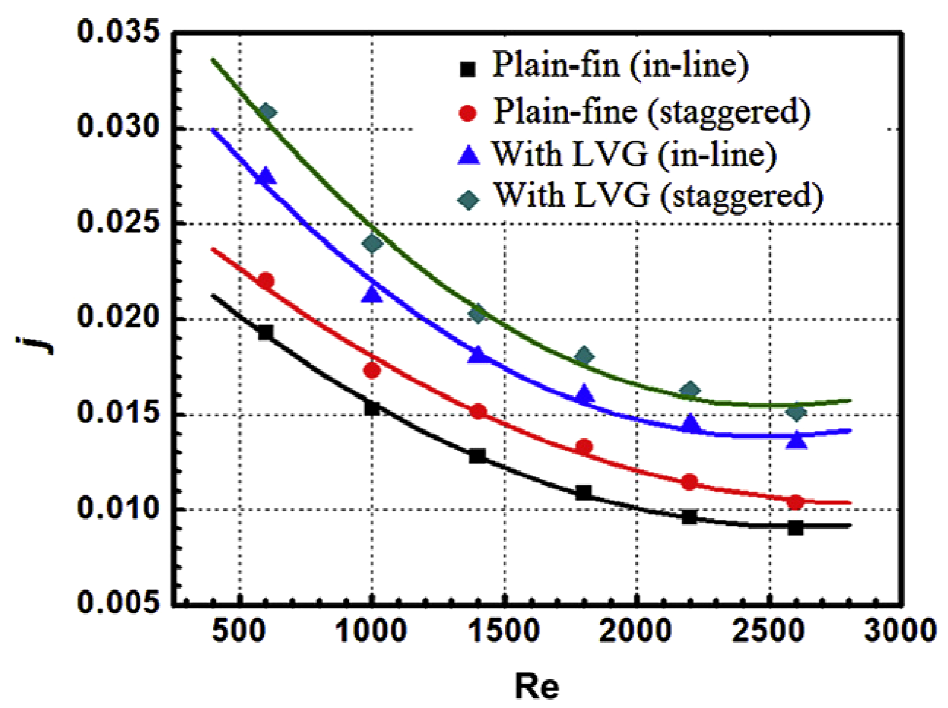

Figure 24 - Colburn factor vs. Reynolds number for aspect ratio 2, Lei et al. (2010). 
In the range of Reynolds number that was studied, the heat transfer for inline arranged fin-tube heat transfer was enhanced $38.8-50.9 \%$, while the heat transfer enhancement for the staggered arrangement was $35.1-45.2 \%$. In other words, the enhancement for inline is better than that for the staggered arrangement.

Figure 25 shows the profile of Friction factor ( $f$ ) as a function of the Reynolds number for different arrangements. Friction factor decreased for increasing Reynolds number, and with the vortex generator exhibited higher drag than for the plain fin. For the fin-tube heat exchanger with side delta winglet, the change of the drag came from two sources. Firstly, the form drag of the delta winglet causes an increase of drag. On the other hand, the delta winglet on the side of the tube resulted in increase of the fluid velocity near the tube and delayed the boundary layer separation; the size of the wake region was decreased so that the form drag on the tube was decreased. Torii et al. (2002) also concluded this, which is explored in Chapter 5. In the range of Reynolds numbers studied, the Friction factor for inline arranged fin-tube heat transfer was increased in 30.3-46.8\%, while the increase of Friction factor for staggered arrangement was 19.3-34.5\%. In Figure 25, an increase of Friction factor due to delta winglet for higher Reynolds number was more significant than for low Reynolds number.

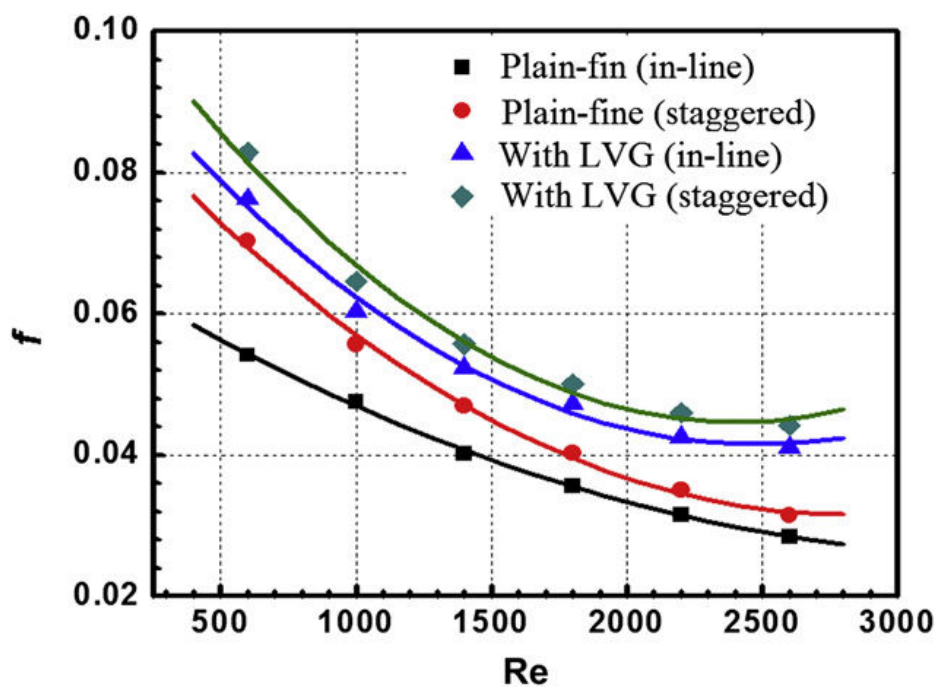

Figure 25 - Friction factor vs. Reynolds number for aspect ratio 2, Lei et al. (2010).

Performance factor (JF) for different tube arrangements are shown in Figure 26. For both inline and staggered arrangements, the overall performances for the cases with 
delta winglets were better than those of the plain fins. This finding indicates that the enhancement of heat transfer was more significant than the increase of the friction factor.

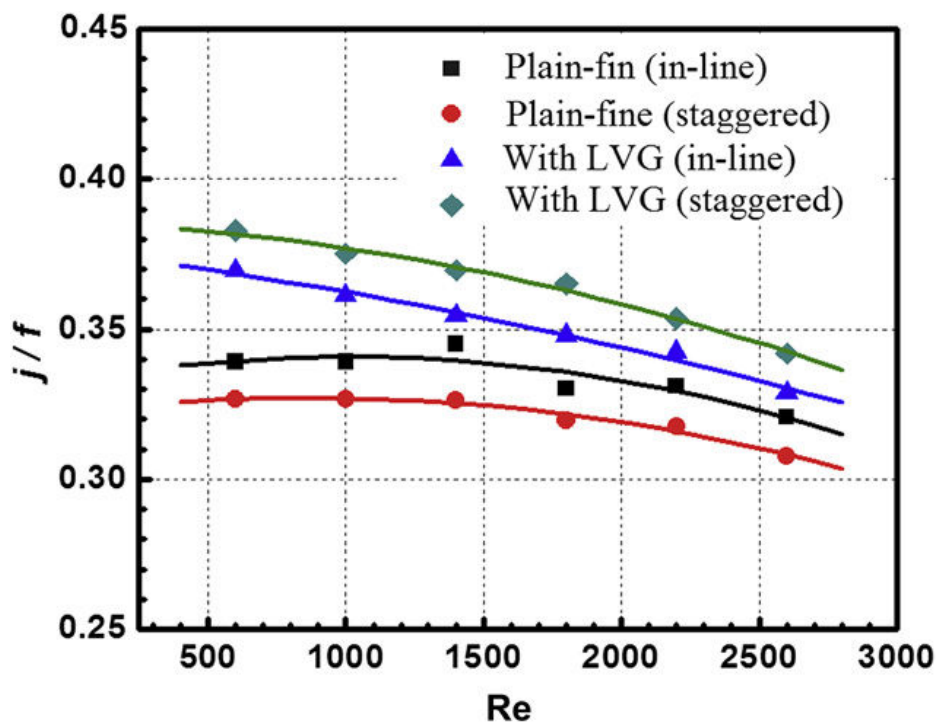

Figure 26 - Heat transfer and drag for different arrangements and aspect ratio 2, Lei et al. (2010).

In order to reveal the effects of attack angle of the delta winglets on heat transfer and pressure drop, performance of fin-and-tube heat exchangers with delta winglets for five different attack angles $\left(\theta=10^{\circ}, 20^{\circ}, 30^{\circ}, 40^{\circ}, 50^{\circ}\right)$ are studied. Figure 27 shows the Colburn factor versus Reynolds number for these five different attack angles. Heat transfer was enhanced for all five cases. For an unchanging Reynolds number, the Colburn factor was increased for increasing attack angle. Increases of the Colburn factor with attack angle were more significant for small attack angle, and such increases became less significant for large attack angle. Figure 28 shows Friction factor versus Reynolds number for these different attack angles, which was increased for all five cases with increasing of the attack angle. These increases were less significant for small attack angle. 


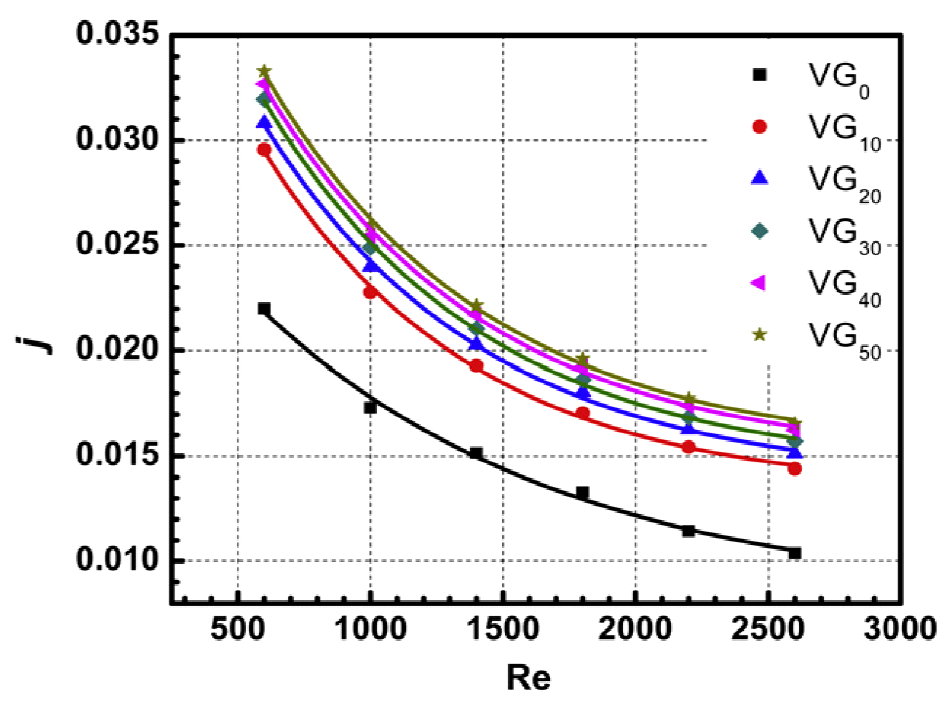

Figure 27 - Colburn factor at different attack angles and aspect ratio 2, Lei et al. (2010).

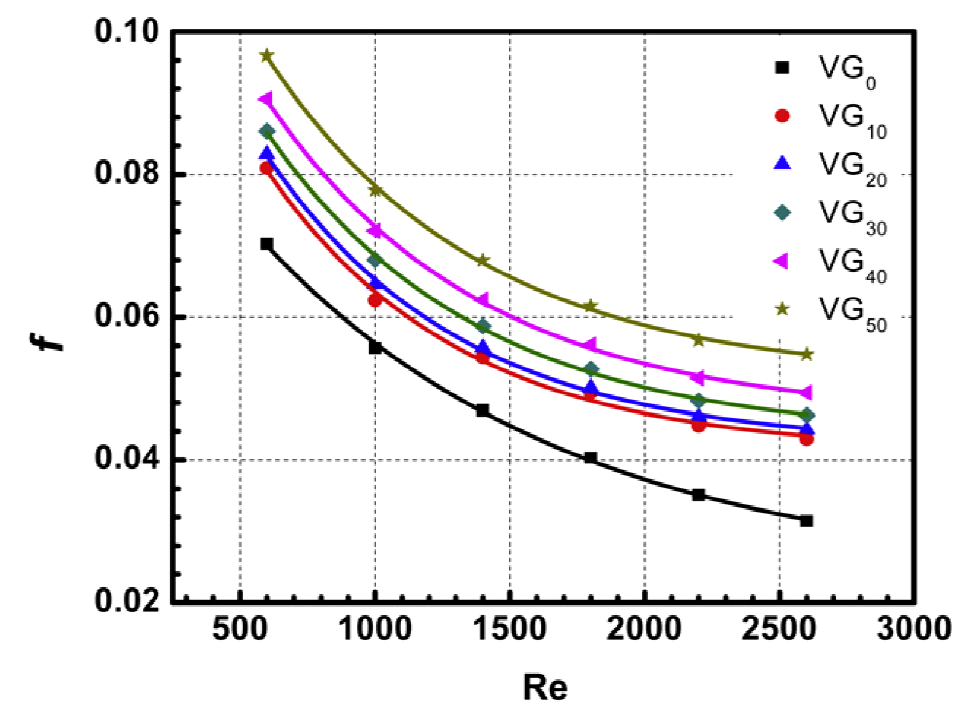

Figure 28 - Friction factor at different attack angles and aspect ratio 2, Lei et al. (2010).

The relation heat transfer-to-drag performances for delta winglets for different attack angles are shown in Figure 29. For the case that the attack angle was $50^{\circ}$, the value of JF factor was greater than the case of plain fin, when the Reynolds number was under 1800. When the Reynolds number was above 1800 , the JF factor value for the case of delta winglet was lower than that of the plain fin. For other attack angles, the overall performances of the cases with delta winglet were better than that of the plain fin for all Reynolds numbers studied. The increase of JF factor with increasing attack angle became less significant with increase of the Reynolds number. The 
computational results also indicated that delta winglet with attack angle of $20^{\circ}$ had the best overall performance.

Effects of the aspect ratio of the delta winglet were also studied, while the attack angle was fixed at $20^{\circ}$. Figure 30 shows the Colburn factor versus Reynolds number for different aspect ratio $(\Lambda)$. Under an unchanged Reynolds number, the Colburn factor increased for increasing aspect ratio, and this became less significant for higher aspect ratio. Figure 31 shows the Friction factor versus Reynolds number for different aspect ratios. The Friction factor increased with increasing aspect ratio and this became more pronounced for higher aspect ratio. The overall performance of heat exchanger with delta winglets for different aspect ratios is shown in Figure 32. For all four aspect ratios studied, the value of JF factor decreased with increasing Reynolds number. The delta winglet with the aspect ratio of 2 was the best relation for thermal-hydraulic performance.

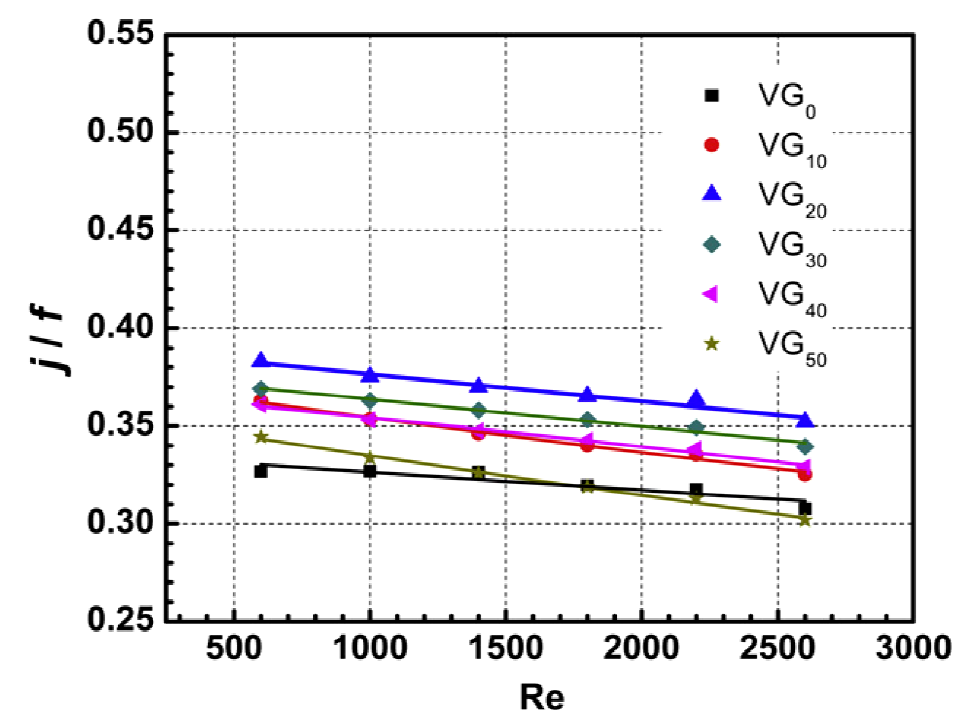

Figure 29 - Performances at different attack angles and aspect ratio 2, Lei et al. (2010). 


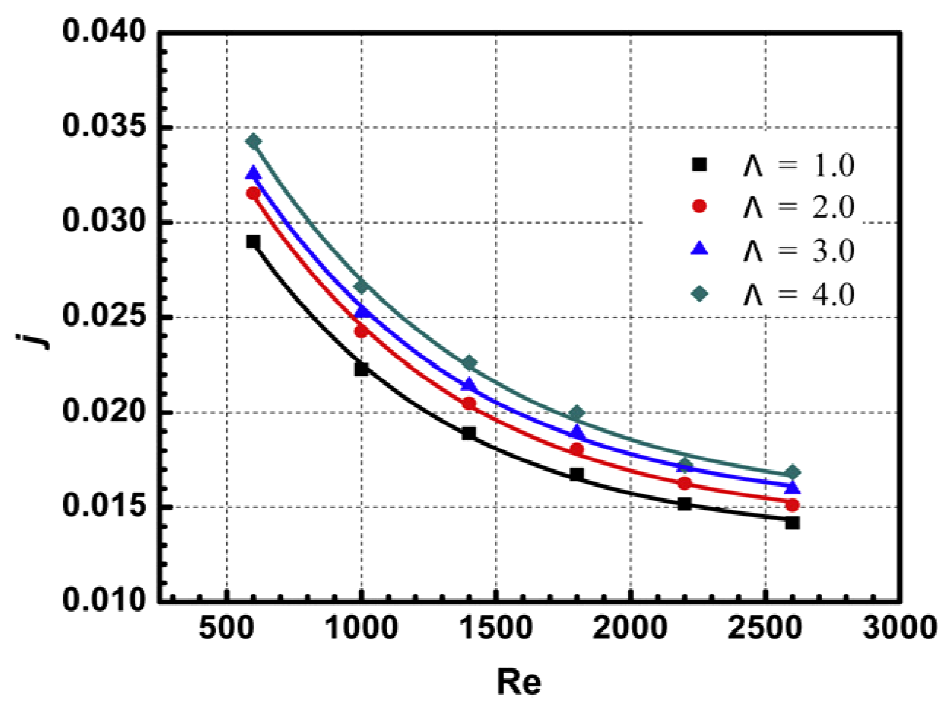

Figure 30 - Colburn factor at different aspect ratios for attack angle of $20^{\circ}$, Lei et al. (2010).

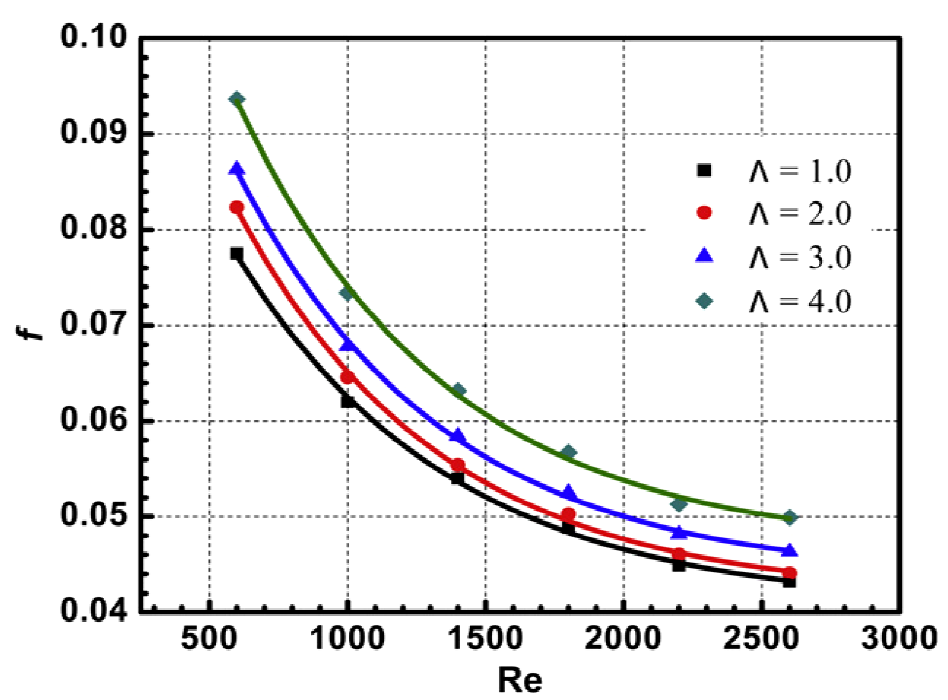

Figure 31 - Friction factor at different aspect ratios for attack angle of $20^{\circ}$, Lei et al. (2010).

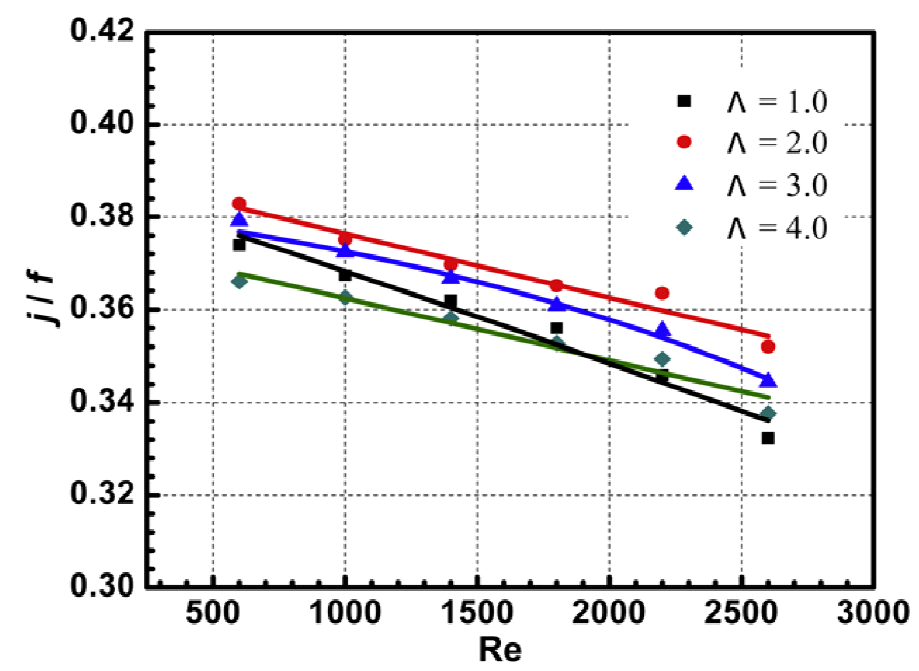

Figure 32 - Performance at different aspect ratios for attack angle of $20^{\circ}$, Lei et al. (2010). 
Torii et al. (2002) proposed a novel technique that can augment heat transfer but nevertheless can reduces pressure-loss in a fin-tube heat exchanger, with circular tubes in a relatively low Reynolds number flow, deploying delta winglet-type vortex generators. The winglets were placed with a heretofore-unused orientation for the purpose of augmentation of heat transfer. This orientation was called as commonflow-up configuration, Figure 33b. The authors assign the "common-flown-down" to work headed by Fiebig et al. (1993). Figure 33 shows the both configuration evaluated by Torii et al. (2002). With common-flow-up configuration (Figure 33b), the winglet pair created constricted passages behind the tube, which brings about separation delay. The fluid accelerates in the constricted passages and the point of flow separation delays downstream. Narrowing of the wake and suppression of vortex shedding are the obvious outcome of such a configuration which reduces form drag. Since the fluid was accelerated in this passage, the zone of poor heat transfer on the fin surface was also removed from the near-wake of the tube. Reynolds number was calculated based on two times channel height from 350 to 2100, and aspect ratio of the vortex generator was 2 . Inline and staggered arrangement were analyzed. For common-flow-up configuration it was considered attack angle of $165^{\circ}$ (or $15^{\circ}$ depending on the reference axis). In case of inline tube banks, the configurations by Fiebig et al. (1993) reported increase of $10 \%$ to $25 \%$ in the heat transfer enhancement with increase of $20 \%$ to $35 \%$ in pressure-loss penalty, in comparison with tube-banks without vortex generators. Common-flow-up configuration proposed by Torii et al. (2002) increased the average heat transfer in $10 \%$ to $20 \%$ and simultaneously decreased the pressure drop in $8 \%$ to $15 \%$. A much better performance was achieved in a staggered tube arrangement with the same configuration of winglet pairs as in the in-line arrangement. In this case, the pressureloss reduction of $34 \%$ to $55 \%$ was achieved together with the heat transfer enhancement of $10 \%$ to $30 \%$. For Reynolds number of 350 , the pressure-loss reduction of $55 \%$ was achieved with heat transfer enhancement of $30 \%$. Kwak et al. (2005) carried out a similar work with three rows of tubes. They achieved the same trend, ie, the delta winglet vortex generator positioned in common-flow-up configuration enhanced the heat transfer and simultaneously decreases the pressure drop. 


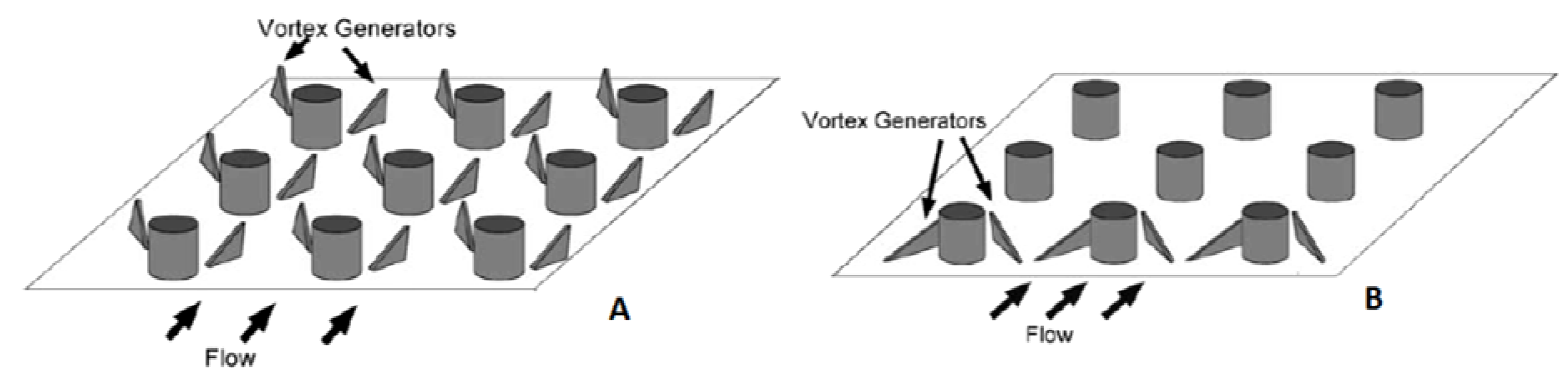

Figure 33 - Configuration of winglet type vortex generator on the fin surface-tube bank: (a) commonflow-down configuration; (b) common-flow-up configuration, Torii et al. (2002).

Here, it is worth comparing the results obtained by Torii et al. (2002) and Lei et al. (2010). Both works considered common-flow-up configuration, however, Torii reported heat transfer enhancement with decrease in pressure-loss penalty, whereas Lei reported heat transfer enhancement with simultaneously increase in drop pressure, although the JF factor was higher than 1 . The best configuration by Torii et al. (2002) and Lei et al. (2010) are faced to the results of this thesis in Chapter 5.

Pesteei et al. (2005) presented an experimental work to determine the best position of the delta winglet pairs for a given aspect ratio. The study was carried out by evaluating local heat transfer coefficient and flow loss in a channel built by parallel plates (fins), with a single cylindrical obstacle (tube). The experiment was conducted with and without delta winglet vortex generators, of height equal to channel height and aspect ratio of 1.33. Five different positions of winglet type vortex generators were evaluated. However, the authors not mentioned the criteria for their choices. The measurements were made for Reynolds number of 2250 and the attack angle was kept constant $\left(\theta=45^{\circ}\right)$. Results showed a substantial increase in the heat transfer with winglet type vortex generators. The average Nusselt number increased about $46 \%$. The best location of the winglets was with $\Delta x=0.5 \mathrm{D}$ and $\Delta y=0.5 \mathrm{D}$. The increase in pressure drop was around $18 \%$. Regarding this work, the researchers gave attention only to heat transfer enhancement and not to JF factor for choice the best configuration. Thus, if this criterion were adopted, the configuration $\Delta \mathrm{X}=0.5 \mathrm{D}$ and $\Delta y=0.6 \mathrm{D}$ would also be a good position. Although the reference position being not clear for $\Delta \mathrm{x}$ and $\Delta \mathrm{y}$, these positions and angle were faced to the present work. 
Wu and Tao (2007) carried out a 3-D numerical simulation for laminar flow heat transfer of the fin-and-tube surface with three rows and delta winglet vortex generators. The effects of Reynolds number (from 800 to 2000) and the attack angle $\left(30^{\circ}\right.$ and $\left.45^{\circ}\right)$ were examined. The phenomenology and trends observed by the authors did not differ from that discussed so far, ie, for inline arrangement of tube banks and vortex generator in common-flow-down configuration, it was reported heat transfer enhancement with simultaneously pressure-loss penalty. The JF factor for attack angle of $45^{\circ}$ was higher than for $30^{\circ}$.

As Torii et al. (2002), Joardar and Jacobi (2008) evaluated vortex generator in common-flow-up configuration. Heat transfer enhancement was experimentally evaluated by full-scale wind-tunnel testing of a compact plain-fin-tube heat exchanger. The effectiveness of a 3 VG alternate-tube inline array of vortex generators was compared to single-row vortex generator design and the baseline configuration. The winglets were placed in common-flow-up orientation for improved tube wake management. The overall heat transfer and pressure drop performance were assessed under dry-surface conditions over a Reynolds number range based on hydraulic diameter from 220 to 960 . The air-side heat transfer coefficient increased from $16.5 \%$ to $44 \%$ for the single-row winglet arrangement, with an increase in pressure drop of less than $12 \%$. For the three-row vortex generator array, the enhancement in heat transfer coefficient increased with Reynolds number from $29.9 \%$ to $68.8 \%$, with a pressure drop penalty from $26 \%$ for $\operatorname{Re}=960$ to $87.5 \%$ for $\operatorname{Re}$ $=220$. These results indicate that vortex generator arrays can significantly enhance the performance of fin-tube heat exchangers with flow depths and fin densities typical to those used in air-cooling and refrigeration applications. Although the configuration common-flow-up was applied, the pressure-loss penalty accompanies the heat transfer enhancement, contrary to that claimed by Torii et al. (2002).

Tang et al. (2009) headed an interesting work that contributes for reinforce the subject of this thesis. Firstly, they experimentally investigated the heat transfer and friction characteristics of five kinds of fin-and-tube heat exchangers, with the number of tube rows $(N=12)$ and the diameter of tubes $\left(D_{0}=18 \mathrm{~mm}\right)$. The optimization of heat exchanger with VGs was also addressed. The test samples consist of five types of fin configurations: crimped spiral fin, plain fin, slit fin, fin with delta-winglet longitudinal vortex generators (VGs) and mixed fin with front 6-row vortex-generator fin and rear 
6-row slit fin, Figure 34. The heat transfer and friction factor correlations for different types of heat exchangers were obtained with the Reynolds numbers ranging from 4000 to 10,000 . For heat exchanger optimization, the trade-off between heat transfer and pressure drop was considered. Optimization technique based on Genetic Algorithm was conducted for heat exchanger with vortex generators. The attack angle, length and height of the vortex generator were considered as input parameters for optimization. Total of 27 models were simulated for the development of correlations for Colburn and Friction factors. In this case, only three parameters of the vortex generator were considered and, therefore, a simple correlation was developed and used in the optimization process. In this thesis, eight parameters were considered and the correlation is more complex expression. Thus, Neural Network Methodology (discussed in Chapter 4 and Appendix B) was employed as surrogate model. Before optimization, for high Reynolds numbers, the heat exchanger with slit fin resulted in the best heat transfer performance. The larger attack angle, higher length and smaller height of vortex generators led to the best overall performance of heat exchangers with VGs. Finally, the optimized vortex-generator fin resulted in the best heat transfer performance than slit fin. These last results showed that the optimization approach was a good way to find the best options in heat transfer enhancement subject.

Lemouedda et al. (2010) investigated the optimal attack angles of the delta-winglets based on the Pareto optimal strategy. The optimization process combined a CFD analysis, Genetic Algorithms (GA) and the Response Surface Methodology (RSM). The attack angle of a pair of delta-winglet-type VGs mounted behind each tube was varied between $-90^{\circ}$ and $+90^{\circ}$. Three circular tube rows with inline and staggered tube arrangements were investigated for Reynolds numbers from 200 to 1200 (based on inlet height and inlet velocity). Figure 35 shows details of the aspect ratio and position of the vortex generator relative to the tube. Depending on the attack angle the configuration becomes either common-flow-down or common-flow-up arrangement. Lemouedda et al. (2010) employed the concept of Pareto optimality, which is an efficient approach to handle multi-objective optimization. This approach is based on the principle of dominance, which states that a solution is clearly better than or dominating another solution, if it is at least better in one objective and better or equal in all the remaining objectives. Thus, the final best compromise solutions 
result by removing all solutions that are dominated by at least one other solution. The outcome of a Pareto optimization is a set of optimal solutions. Usually the decisionmaker (the designer) is able to choose a final solution from these non-dominated points using further considerations. Pareto frontier not necessarily deals for maximum JF factor but just deals for dominate designs.

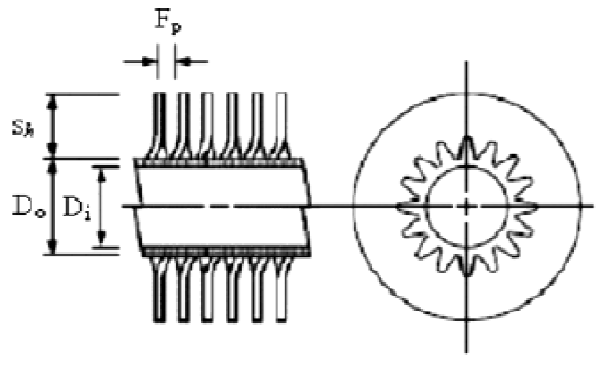

(a) Crimped spiral fin

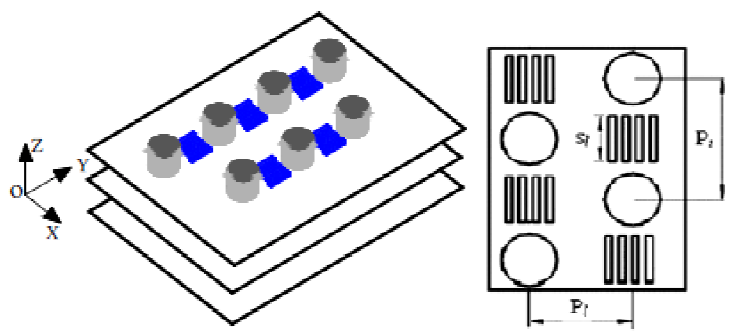

(c) Slit fin

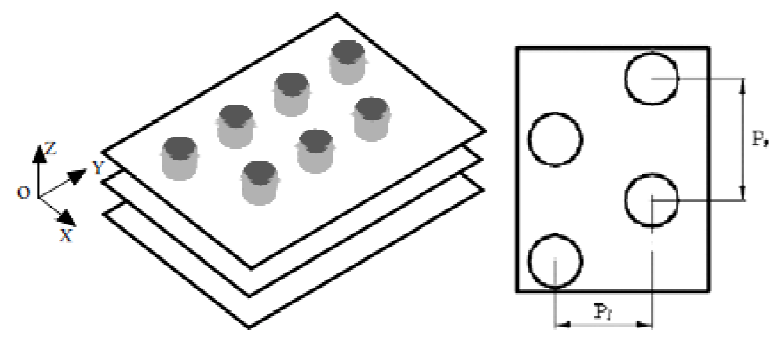

(b) Plain fin

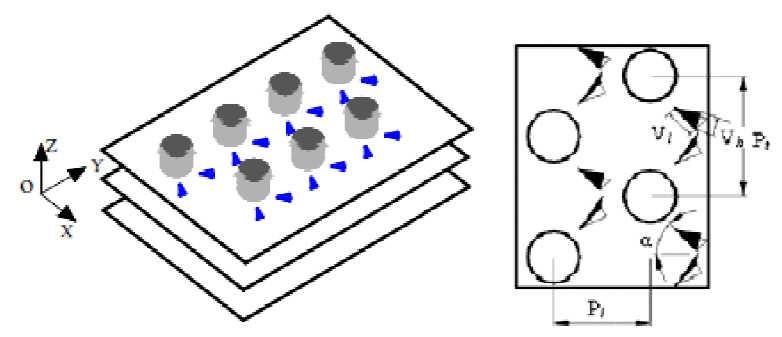

(d) Vortex-generator fin

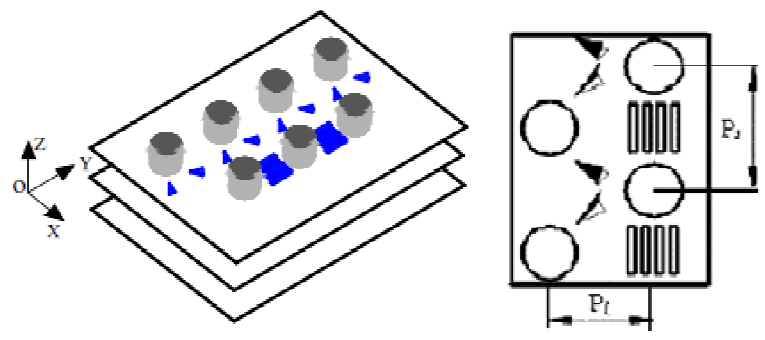

(e) Mixed fin

Figure 34 - Five types of fin configurations, Tang et al. (2009). 


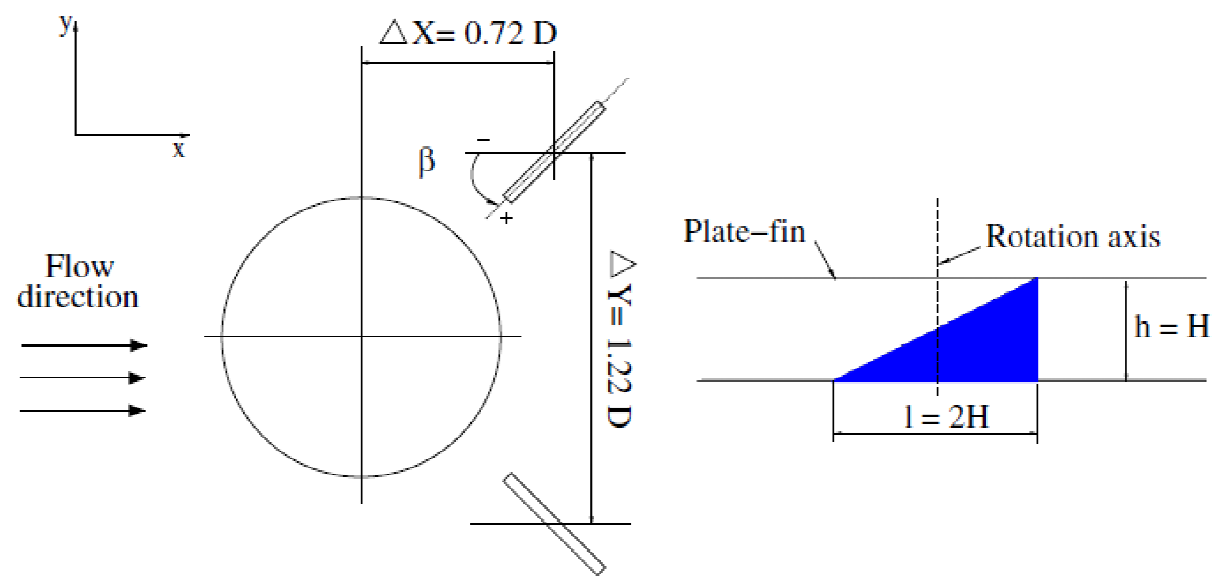

Figure 35 - Geometric configuration of the delta-winglets in the elementary model used by Lemouedda et al. (2010)

Lemouedda et al. (2010) considered only two input parameters due to the high calculation time needed for the simulations performed (Reynolds number and attack angle of the delta-winglet). Their suggestion was, in part, the main goal of this thesis, ie, although the study considered only two input parameters, the methodology can be extended to other parameters, such as: the position of the delta-winglets, both streamwise and transverse tube spacing, as well as, other structural parameters. For inline arrangement the common-flow-down configuration was found to be more suitable than the common-flow-up. Inline and staggered tube arrangements performances were compared with and without winglets. The staggered arrangement was better than the inline one for both cases with and without winglets. The attack angle was equals to $+60^{\circ}$ (common-flow-down configuration) and $-60^{\circ}$ (common-flowup configuration), which reported a good result for objective functions. Finally, the authors pointed out the benefit of adopting optimization process for other kind of engineering applications with large design space. Furthermore, the combination of the CFD tool, Genetic Algorithms and RSM, considerably decreased the number of CFD computations needed.

A good work that differs a little bit of the traditional researches was conducted by $\mathrm{Wu}$ and Tao (2011). To achieve heat transfer enhancement and lower pressure loss penalty, two novel fin-tube surfaces with two rows of tubes in different diameters were presented in their paper. Numerical simulation results showed that the fin-tube surface with the first row tube in smaller size and the second row tube in larger size 
led to an increase of heat transfer and decrease of pressure drop, in comparison with the traditional fin-tube surface with two rows of tubes in the same size. Based on this understanding, delta winglet pairs were punched out only from the larger fin area around the first transverse row of tubes, in smaller size in the novel fin-tube surfaces. Delta winglet pairs were arranged either in common-flow-up or common-flow-down configurations. The operating condition of the heat exchanger was according to the found in refrigeration or/and air conditioning system. Thus, the operating range of the frontal air velocity was between 2.5 and $5.5 \mathrm{~m} / \mathrm{s}$ and, therefore, the Reynolds number range was determined for 800-2000, based on the hydraulic diameter. The authors discussed the effects explained by Torii et al. (2003), eg, the common-flow-up arrangement accelerate the flow between tube and vortex generator, decreasing the drag on the tube. Overall, the authors conclude that the fin-tube surface with the first row tubes in smaller size and the second row tubes in larger size can lead to an increase of heat transfer and decrease of pressure drop in comparison with the traditional fin-tube surface with two rows of tubes in the same size. The novel fin-tube surface with two rows of tubes in different diameter and delta winglet pairs, punched out only from the larger fin area around the first transverse row of tubes in smaller size, arranged either in common-flow-up or common-flow-down configurations, provide a further heat transfer enhancement and pressure drop reduction through the careful arrangement of the location, size and attack angle of the delta winglet pairs. The common-flow-down configuration was more attractive for heat transfer enhancement, whereas the common-flow-up configuration was an appropriate choice for pressure loss reduction. And finally, the last conclusion from the authors reinforces the theme chosen for this thesis, ie, the researchers suggest that more work about the parameters optimization of the delta winglet ought be carried out in practical heat exchanger. As sequence of this work, Wu et al. (2012) experimentally investigated the same approach with rows of tubes in different diameters.

Hwang et al. (2012) investigated the augmentation heat transfer in compact heat exchanger through delta vortex generator. After 10 years, the researchers found the same conclusions by Torii et al. (2002), ie, in compact heat exchanger was possible to reach heat transfer enhancement accompanied for pressure drop reduction, considering common-flown-up configuration. 
Recently, the main researches have been walked to apply vortex generator together other passive techniques namely compound methods, ie, vortex generator and wavy fin could be applied to augmentation the heat transfer in a compact heat exchanger. For example, Gong et al. (2013) numerically investigated the air-side heat transfer and fluid flow characteristics of wavy fin-and-tube heat exchanger, punched with combined rectangular winglet pairs. The influences of several main parameters of the combined configuration on heat transfer and friction were examined and compared to rectangular winglet pairs. The numerical results showed that, compared to the rectangular winglet pair, the vortices generated for combined configuration were larger and stronger.

Hence, combined configuration was more effectively to improve heat transfer and increase friction. Huisseune et al. (2013) also investigated the compound techniques. They claim that by punching delta winglet vortex generators in the louvered fins, the size of the tube wakes could be reduced. The authors numerically evaluated the influence of the louver and delta winglet geometry on the thermal and hydraulic performance of a heat exchanger. Compound techniques sounds like an interesting option to enhancement heat transfer in heat exchanger, and the methodology applied in this thesis could be used to evaluate this in the future.

\subsection{Critical appraisal of the previous works}

Compared to the traditional heat transfer enhancement techniques, the longitudinal vortex generator shows significant advantages. While the capability of heat transfer is significantly enhanced, the pressure drop only increases by a small degree, or even decreases in some cases, eg, for louver fin, the criterion $J F=\left(j / j_{0}\right) /\left(f / f_{0}\right)>1$ is very difficult to achieve. The main goal of the optimization process, herein, is to find optimal vortex generator arrangement to meet different objective functions, described in Chapter 4. Each objective function evaluated here is based on Colburn factor (j) and Friction factor (f). In Chapter 5, the optimization is achieved with unusual VG configuration, which was not studied by current researches, yet. 
Heat transfer enhancement by longitudinal vortex generator is affected by many factors, such as attack angle, aspect ratio, locations and arrangement. Different "optimized" structures exist depending of the CHE operating conditions. The word "optimized" was marked in the previous sentence, because the optimization process was not appropriated made in current researches. In fact, just few configurations are evaluated, eg, some attack angles are evaluated for the same position and the better is chosen as the "optimized" configuration. For another vortex generator position, the same work is conducted and, therefore, there is the possibility that a different attack angle could be the better choice. Thus, the main differences among the works are the operation conditions and the isolated study of the parameters. However, the effect of these different parameters (for example, attack angle and position) on $\mathrm{CHE}$ performance is singly performed, since the parameter space for the optimization of longitudinal vortex generator is huge and, thus, the experimental investigations cannot provide an optimized design. Therefore, using numerical approaches to carry out studies on design optimization of heat exchangers seems to be mandatory.

Overall, on the basis of the outlined review about researches on heat transfer enhancements by longitudinal vortex generators in fin-tube heat exchanger, is evident that the position and attack angle need to be simultaneously optimized. He and Zhang (2012) called it as the "hot spots" for future research. Moreover, herein, it is proposed a new VG parameter, which we called by "Roll angle". 


\section{Modeling and Numerical method}

This chapter describes the heat exchanger model and the performance parameters used for characterizing the heat transfer and dynamic of the flow. The model heat exchanger for this project is presented, besides information about the heat exchanger, fin-and-tube efficiency, pressure drop, and the dimensionless groups used in the calculation process. The numerical methodology also is presented.

\subsection{Governing Equations}

In developing the model, the following assumptions are made:

1. Constant fluid properties (density, viscosity, specific heat and thermal conductivity of fluid);

2. Steady state regime;

3. Incompressible flow;

4. Body force and the dissipation term neglected.

The compact forms of the governing equations for this problem are the three-dimensional continuity, momentum, energy, and scalar transport equations and can be written (generally) as follows: 
- Continuity equation:

$$
\frac{\partial\left(\rho u_{i}\right)}{\partial x_{i}}=0
$$

- Momentum equation:

$$
\frac{\partial}{\partial x_{i}}\left(\rho u_{i} u_{j}\right)=\frac{\partial}{\partial x_{i}}\left(\mu \frac{\partial u_{j}}{\partial x_{i}}\right)-\frac{\partial p}{\partial x_{j}}
$$

- Energy equation:

$$
\frac{\partial}{\partial \mathbf{x}_{i}}\left(\rho u_{i} T\right)=\frac{\partial}{\partial \mathbf{x}_{i}}\left(\frac{k}{c_{p}} \frac{\partial u_{j}}{\partial x_{i}}\right)
$$

- General transport equation (for scalars):

$$
\frac{\partial\left(\rho u_{i} \phi\right)}{\partial \mathbf{x}_{i}}=\frac{\partial}{\partial \mathbf{x}_{i}}\left[\Gamma_{\phi} \frac{\partial \phi}{\partial \mathbf{x}_{i}}\right]+S_{\phi}
$$

where $\Gamma_{\phi}$ and $S_{\phi}$ are the diffusion coefficient and source term, respectively, for each of the $\mathrm{N}$ scalar equation.

A commercial CFD (Computational Fluid Dynamics) code (Ansys Fluent) was applied based on the Finite Volume Methodology, which can be seen in details in Maliska (2004). A staggered grid using solvers for turbulent flow was solved, using the Reynolds Averaged Navier-Stokes equations (RANS), considering the closure SST komega turbulence model. To ensure coupling between velocity and pressure, the Coupled Algorithm was used. The $2^{a}$ Upwind scheme was employed for the discretization of the convective term. Next sections present a brief study about the 
turbulence models and coupling algorithm, especially because the most reference in open literature apply either standard k-epsilon or RNG k-epsilon turbulence model, beyond SIMPLE/SIMPLEC coupling algorithm.

\subsection{Turbulence Model}

Reynolds number for the heat exchanger flow in this project is 250 and 1400, which means that the flows can be laminar, transitional, or turbulent at different flow velocities. In this project, three turbulence models (standard/RNG k-epsilon and SST k-omega) were evaluated in order to investigate which is the best for the different types of the dynamic flow. This section describes some of the commonly used turbulence models in compact heat exchanger, which the key features were showed.

Table 3 lists some commonly used turbulence models. There are a large number of different turbulence models. The turbulence models listed below are based on the Reynolds averaged Navier-Stokes (RANS) equations.

Table 3 - Commonly turbulence models, Versteeg and Malalasekera (2007).

\begin{tabular}{llll}
\hline Turbulence Model & Flow & Model Type* & \\
\hline Spalart-Allmaras & C, I & One-equation & \\
k-epsilon standard & C, I & Two-equation & More Physics \\
k-epsilon RNG & C, I & Two-equation & \\
Wilcox k-omega & C, I & Two-equation & \\
Menter SST k-omega & C, I & Two-equation & \\
k-epsilon Realizable & C, I & Non-linear & Higher Accuracy \\
LRR R-epsilon & C, I & RSM & \\
Launder-Gibson R-epsilon & C, I & RSM & \\
Lien k-epsilon & I & Cubic & \\
Lien low Re k-epsilon & I & Cubic & \\
Shih k-epsilon & I & Quadratic & \\
\hline
\end{tabular}

*Number of extra transport equations to be solved along with the RANS equations. $\mathrm{C} \rightarrow$ Compressible, I $\rightarrow$ Incompressible.

Moreover, others methods could be used to predict the turbulence (besides those RANS models listed in Table 3). LES (Large Eddy Simulation) solves for the motion of the larger eddies while DNS (Direct Numerical Simulation, the most accurate and time-consuming/computationally expensive method of all for calculating turbulence) 
solves for all motion using the Navier-Stokes equations. LES and DNS are computationally very expensive due to requirements including very fine 3D mesh and transient behavior. Due to hardware requirements, LES and DNS were not used for this project, especially when optimization approach is applied, because a large count of the analysis was necessary to find optimal solution. This is discussed in Chapter 4 and Appendix B.

In this project, the turbulence models evaluated were the standard/RNG k-epsilon and SST k-omega. Firstly, a brief explanation about why other turbulence models were ruled out for this project is presented, and then the basic turbulence concepts about standard/RNG k-epsilon and SST k-omega models are presented.

To decide which turbulence models to use for this project, considerations were taken regarding relevance to the particular type of flow involved (applicability), complexity of the physics and time for computing, whether the model is compressible or incompressible flow and availability in commercial solver, beyond of accuracy.

Spallart-Allmaras is one-equation mixing length model, which solves for the turbulent kinematic viscosity using a modeled transport equation. This model specifies the eddy length scale with an algebraic formula, which was firstly designed for external aerodynamic boundary layer economical computations. This model is known for not accurately describing flows involving separation and recirculation. This project involves internal flow with both separation and recirculation, making this model inappropriate for this purpose.

Realizable k-epsilon model is a non-linear version of the k-epsilon model. This model retains the two-equation, but expands the model by including additional effects to account for Reynold's stress anisotropy, without actually using the seven extra equations used in the RSM, for exactly modeling the Reynolds stresses. The turbulent viscosity expression and the rate of dissipation of kinetic energy equation for standard k-epsilon model are both changed in the realizable k-epsilon model, to take into account that turbulence not always adjust itself instantaneously, while moving through the flow domain, meaning that the Reynolds stress is partially dependent on the mean strain rate itself. This means that the nonlinear realizable kepsilon model allows for the phenomena of the state of turbulence lagging behind the changes disturbing the turbulence production and dissipation balance. This 
turbulence model was not used on this project, but could be considered for future work with this type of heat exchangers to account for anisotropic Reynold's stresses in the flow. No work in open literature was found which applied this turbulence model.

Reynold's Stress Equation Models (RSM): LRR R-epsilon and Launder-Gibson $R$ epsilon models, calculates the anisotropic Reynolds stresses present in typical flows of complex strain fields or significant body forces. RSM is the most complex of the turbulence models, and was designed to address the problems of the k-epsilon model (which cannot predict flows in long non-circular duct, because of the isotropic modeling of the Reynold's stresses). The RSM can, therefore, accurately account for the Reynold's stress field directional effects. Because of the many Reynold's stresses to model, there are seven extra partial differential equations to solve, making computing costs very high. RSM is not as widely validated as the k-epsilon model and was not used in this project due to the computing requirements, although seems to be an interesting continuation for this project in the future, since the long thin channels between the heat exchanger fins probably means the Reynold's stresses in the heat exchanger are anisotropic.

Overall, considering the background from researches over the last year, the turbulence hypothesis and the mean phenomenon verified in enhancement heat transfer (discussed in Chapter 1 and 2), and the CFD current researches in CHE, we decided to evaluate only the standard/RNG k-epsilon and SST k-omega turbulence models.

\subsubsection{RANS approach}

In RANS approach, the flow is separated into mean and fluctuating components,

$$
\mathrm{U}=\overline{\mathrm{U}}+\mathrm{u}^{\prime}
$$

Equation 7 can graphically be represented according to Figure 36 . The time averaging velocity component is defined as: 


$$
\overline{\mathrm{U}}=\frac{1}{\mathrm{~T}} \int_{0}^{\mathrm{T}} \mathrm{Udt}
$$

where $T$ is the averaging time of the simulation, usually chosen to be large, if compared to the typical timescale of the turbulent fluctuation.

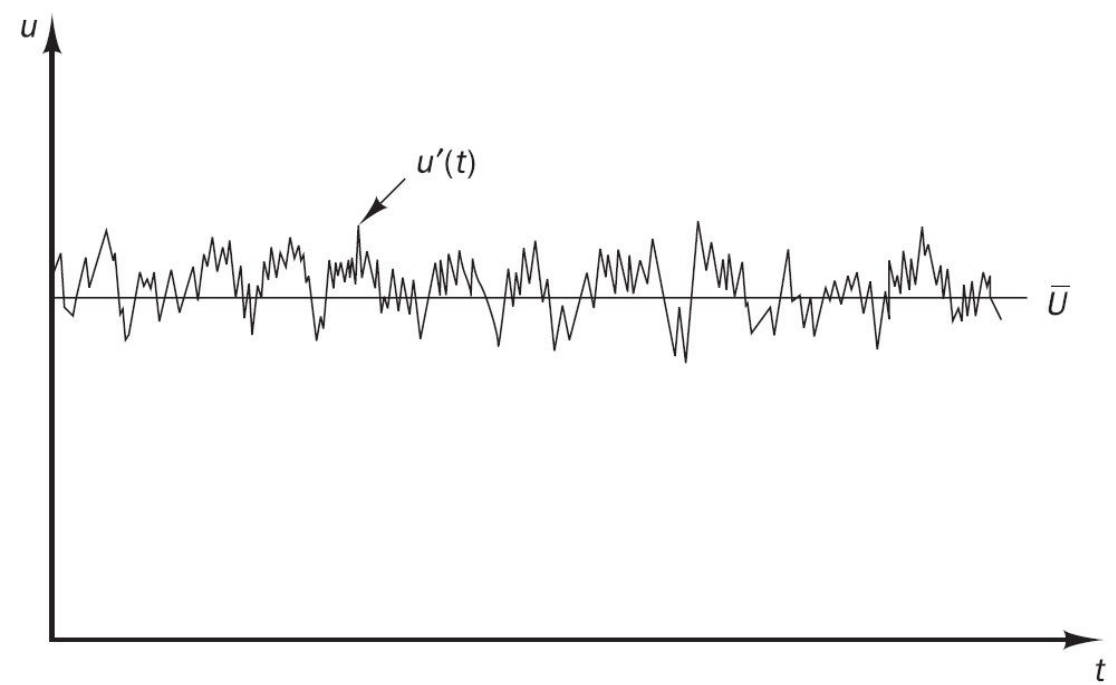

Figure 36 - Time averaging of turbulence used on RANS models.

Reynolds Averaged Navier-Stokes (RANS) equations are formed by taking the mean of the Navier-Stokes equations using the decomposition shown in Equation 7. Thus, substituting the Equation 7 into the Navier-Stokes for time averaging, one obtains the time averaged Navier-Stokes equations,

$$
\frac{\partial \bar{u}_{i}}{\partial t}+\bar{u}_{j} \frac{\partial \bar{u}_{i}}{\partial x_{j}}=-\frac{1}{\rho} \frac{\partial \bar{p}}{\partial x_{i}}+\frac{\partial}{\partial x_{j}}\left(v \frac{\partial \bar{u}_{i}}{\partial x_{j}}-\overline{u_{i} u_{j}}\right)
$$

where $v$ is the effective viscosity of the flow.

The momentum equation for the mean flow field contains an additional term that originates from the average of the nonlinear term $\left(\overline{u_{i} u_{j}}\right)$, which are the correlation between the fluctuating velocity components. That term is called Reynolds Stress. The existence of the Reynolds stress means that there is no longer a closed set of 
equations, and turbulence model assumptions are needed to estimate the unknowns to solve this closure problem.

Turbulence modeling in engineering CFD methods is a matter of finding mathematical expressions for the Reynolds stress tensor in terms of known quantities. Its complexity ranges from purely algebraic relations to relations that consider one or several extra transport equations for different turbulent correlations.

The transport equation for the Reynolds stress tensor may be derived from the Navier-Stokes equations,

$$
\frac{D \overline{u_{i} u_{j}}}{D t}=d_{i j}+P_{i j}+\varphi_{i j}+\varepsilon_{i j}
$$

where: $\frac{D}{D t} \equiv \frac{\partial}{\partial t}+u_{j} \frac{\partial}{\partial x_{j}}$ is the material derivative, $d_{i j}$ is the diffusion term, $P_{i j}$ is the production term, $\varphi_{\mathrm{ij}}$ is the redistribution term and $\varepsilon_{\mathrm{ij}}$ is the dissipation term.

The equation for production term is given by,

$$
P_{i j}=-\overline{u_{i} u_{k}} \frac{\partial \bar{u}_{j}}{\partial x_{k}}-\overline{u_{j} u_{k}} \frac{\partial \bar{u}_{i}}{\partial x_{k}}
$$

Redistribution term is responsible for driving turbulence toward the isotropic state and a simple model for redistribution term can be written as,

$$
\varphi_{i j}=-c \frac{\varepsilon}{k}\left(\overline{u_{i} u_{j}}-\frac{2}{3} k \delta_{i j}\right)
$$

where $\mathrm{k}$ is the turbulent kinetic energy.

The dissipation term occurs at the smallest scales (Kolmogorov scales), at which eddies are isotropic, and this implies, 


$$
\varepsilon_{\mathrm{ij}}=\frac{2}{3} \varepsilon \delta_{\mathrm{ij}}
$$

Several approaches already exist to model the Reynolds stress tensor: (i) Reynolds Stress Transport Models (RSMs); (ii) Algebric Stress Models (ASMs) and; (iii) Eddy Viscosity Models (EVMs). Only eddy viscosity models was addressed in this work.

These models are based on Boussinesq hypothesis, which assumes that the Reynolds stresses are related to the mean velocity gradients by the following empirical formula,

$$
\overline{u_{i} u_{j}}=\frac{2}{3} k \delta_{i j}-v_{t}\left(\frac{\partial \bar{u}_{i}}{\partial x_{j}}+\frac{\partial \bar{u}_{j}}{\partial x_{i}}\right)
$$

where $v_{t}$ is the turbulent viscosity. It is important emphasize that the Equation 14 is only valid for incompressible fluid.

In this approach, the turbulent viscosity is introduced in the equation and it must be calculated. Basically, there are three models to determine $v_{t}$ : Zero-Equation Models, One-Equation Models and Two-Equation Models. In this work, only Two-Equation Models was evaluated ( $k-\omega$ and $k-\varepsilon$ models). These models are commonly used on industrial and engineering applications due to relative low computer power combined with robustness on heat transfer results.

\subsubsection{Standard k-epsilon turbulence model}

Two-equation turbulence models allow the determination of both, a turbulent length and time scale by solving two separate transport equations. The standard k-epsilon model in Ansys Fluent falls within this class of models and has become the workhorse of practical engineering flow calculations in the time since it was proposed by Launder and Spalding (1972). Robustness, economy, and reasonable accuracy 
for a wide range of turbulent flows explain its popularity in industrial flow and heat transfer simulations. It is a semi-empirical model, and the derivation of the model equations relies on phenomenological considerations and empiricism. The standard k-epsilon model is a model based on model transport equations for the turbulence kinetic energy $(k)$ and its dissipation rate $(\varepsilon)$. The model transport equation for $k$ is derived from the exact equation, while the model transport equation for $\varepsilon$ was obtained using physical reasoning and bears little resemblance to its mathematically exact counterpart. In the derivation of the standard k-epsilon model, the assumption is that the flow is fully turbulent, and the effects of molecular viscosity are negligible. The standard k-epsilon model is therefore valid only for fully turbulent flows.

This model calculates the turbulent kinetic energy $(k)$ and its dissipation rate $(\varepsilon)$ from the transport equations given by,

$$
\begin{gathered}
\frac{D k}{D t}=\frac{\partial}{\partial x_{i}}\left[\left(v+\frac{v_{t}}{P_{r_{k}}}\right) \frac{\partial k}{\partial x_{i}}\right]+G_{k}+G_{b}-\varepsilon \\
\frac{D \varepsilon}{D t}=\frac{\partial}{\partial x_{i}}\left[\left(v+\frac{v_{t}}{P_{\varepsilon}}\right) \frac{\partial \varepsilon}{\partial x_{i}}\right]+C_{1 \varepsilon} \frac{\varepsilon}{k}\left(G_{k}+C_{3 \varepsilon} G_{b}\right)-C_{2 \varepsilon} \frac{\varepsilon^{2}}{k}
\end{gathered}
$$

where $G_{k}$ term is the generation of turbulent kinetic energy by the mean velocity gradients, $G_{b}$ is the generation of turbulent kinetic energy by buoyancy, $\operatorname{Pr}_{k}$ and $\operatorname{Pr}_{\varepsilon}$ are the Prandtl number for $\mathrm{k}$ and $\varepsilon . \mathrm{C}_{1 \varepsilon}, \mathrm{C}_{2 \varepsilon}, \mathrm{C}_{3 \varepsilon}$ are constants.

The turbulent kinetic energy dissipation rate is,

$$
\varepsilon=\frac{\mathrm{k}^{\frac{3}{2}}}{\mathrm{~L}}
$$

And, for this model, the turbulent viscosity is calculated as, 


$$
v_{t}=C_{v} \frac{k^{2}}{\varepsilon}
$$

where $C_{v}$ is equal to 0.09 for widely practical applications, including for compact heat exchangers design.

As the strengths and weaknesses of the standard model have become known, modifications have been introduced to improve its performance. RNG k-epsilon model is one of these variants that are available in Ansys Fluent.

\subsubsection{RNG k-epsilon turbulence model}

RNG k-epsilon model was derived using a statistical technique (called ReNormalization Group Theory). It is similar in form to the standard k-epsilon model, but includes the following refinements, Ansys Fluent (2010):

- The RNG model has an additional term in its $\varepsilon$ equation, that improves the accuracy for rapidly strained flows.

- The effect of swirl on turbulence is included in the RNG model, enhancing accuracy for swirling flows.

- The RNG k-epsilon theory provides an analytical formulation for turbulent Prandtl numbers, while the standard $\mathrm{k}$-epsilon model uses user-specified, constant values.

- While the standard k-epsilon model is a high-Reynolds-number model, the RNG theory provides an analytically derived differential formulation for effective viscosity that accounts for low-Reynolds-number effects. Effective use of this feature DoEs, however, depend on an appropriate treatment of the near-wall region. It is discussed in the next section. 
These features make the RNG k-epsilon model more accurate and reliable for a wider class of flows than the standard k-epsilon model.

The RNG k-epsilon turbulence model is derived from the instantaneous NavierStokes equations, using a mathematical technique called "Renormalization Group" (RNG) methods.

The analytical derivation results in a model with constants different of those shown in the standard $\mathrm{k}-\varepsilon$ model, and additional functions and terms were included in the transport equations for $\mathrm{k}$ and $\varepsilon$. The equations for $\mathrm{k}$ and $\varepsilon$ are given by,

$$
\begin{gathered}
\frac{D k}{D t}=\frac{\partial}{\partial \mathbf{x}_{i}}\left[\left(\frac{v_{t}}{P r_{k}}\right) \frac{\partial k}{\partial x_{i}}\right]+G_{k}+G_{b}-\varepsilon \\
\frac{D \varepsilon}{D t}=\frac{\partial}{\partial \mathbf{x}_{i}}\left[\left(\frac{v_{t}}{P r_{\varepsilon}}\right) \frac{\partial \varepsilon}{\partial x_{i}}\right]+C_{1 \varepsilon} \frac{\varepsilon}{k}\left(G_{k}+C_{3 \varepsilon}+G_{b}\right)-C_{2 \varepsilon} \frac{\varepsilon^{2}}{k}-R_{\varepsilon}
\end{gathered}
$$

where the term $R_{\varepsilon}$ is the rate of strain term and calculated as,

$$
\mathrm{R}_{\varepsilon}=\frac{\mathrm{C}_{v} \eta^{3}\left(1-\frac{\eta}{\eta_{0}}\right)}{\left(1+\beta \eta^{3}\right)} \frac{\varepsilon^{2}}{\mathrm{k}}
$$

where $\eta_{0}=4.38$ and $\beta=0.012$. Here, $\eta$ is defined as,

$$
\eta \equiv \frac{\mathrm{k}}{\varepsilon} \mathrm{S}_{\mathrm{ij}}
$$

The turbulent viscosity expression for the RNG $\mathrm{k}-\varepsilon$ turbulence model is,

$$
v_{\mathrm{t}}=v\left[1+\left(\frac{\mathrm{C}_{v}}{v}\right)^{\frac{1}{2}} \frac{\mathrm{k}}{\varepsilon^{\frac{1}{2}}}\right]^{2}
$$

with $C_{v}=0.0845$. 
A more comprehensive and detailed description of RNG theory and its application to turbulence can be found in Orszag et al. (1993).

\subsubsection{SST k-omega turbulence model}

SST k-omega model is based on Standard k-omega model, which is based on the Wilcox (1994) k- $\omega$ model, which incorporates modifications for low-Reynoldsnumber effects, compressibility, and shear flow spreading. One of the weak points of the Wilcox model is the sensitivity of the solutions to values for $k$ and $\omega$ outside the shear layer (free stream sensitivity). The standard k-omega model is an empirical model based on model transport equations for the turbulence kinetic energy $(k)$ and the specific dissipation rate $(\omega)$, which can also be thought of as the ratio of $\varepsilon$ to $k$, Wilcox (1994).

The $\mathrm{k}-\omega$ transport equations are given by,

$$
\begin{aligned}
& \frac{\mathrm{Dk}}{\mathrm{Dt}}=\frac{\partial}{\partial \mathrm{x}_{\mathrm{j}}}\left[\Gamma_{\mathrm{k}} \frac{\partial \mathrm{k}}{\partial \mathrm{x}_{\mathrm{j}}}\right]+\mathrm{G}_{\mathrm{k}}+\mathrm{Y}_{\mathrm{k}} \\
& \frac{\mathrm{D} \omega}{\mathrm{Dt}}=\frac{\partial}{\partial \mathrm{x}_{\mathrm{j}}}\left[\Gamma_{\omega} \frac{\partial \omega}{\partial \mathrm{x}_{\mathrm{j}}}\right]+\mathrm{G}_{\omega}-\mathrm{Y}_{\omega}
\end{aligned}
$$

where $G_{k}$ term is the generation of turbulent kinetic energy by the mean velocity gradients, $G_{\omega}$ is the generation of specific dissipation rate, $Y_{k}$ and $Y_{\omega}$ represents the dissipation of $k$ and $\omega$ quantities due to turbulence, and $\Gamma_{k}$ and $\Gamma_{\omega}$ are the effective diffusivity of $k$ and $\omega$, respectively.

The generation of $\mathrm{k}$ and $\omega$ are calculated as follows, 


$$
\begin{aligned}
& G_{k}=-\rho \overline{u_{i} u_{j}} \frac{\partial u_{j}}{\partial x_{i}} \\
& G_{\omega}=\alpha \frac{\omega}{k} G_{k}
\end{aligned}
$$

where the coefficient $\alpha$ is,

$$
\alpha=\frac{\alpha_{\infty}}{\alpha^{*}}\left(\frac{\alpha_{0}+\operatorname{Re}_{\mathrm{t}} / \mathrm{Re}_{\omega}}{1+\operatorname{Re}_{\mathrm{t}} / \mathrm{Re}_{\omega}}\right)
$$

where $\operatorname{Re}_{\omega}=2.95 \alpha^{*}$ and the coefficient $\alpha^{*}$, which damps the turbulent viscosity causing a low-Reynolds-number correction, is given by,

$$
\alpha^{*}=\alpha_{\infty}^{*}\left(\frac{\alpha_{0}^{*}+\operatorname{Re}_{t} / \operatorname{Re}_{k}}{1+\operatorname{Re}_{t} / \operatorname{Re}_{k}}\right)
$$

where: $\operatorname{Re}_{k}=6, \beta_{i}=0.072, \alpha_{0}^{*}=\beta_{i} / 3$ and $\operatorname{Re}_{t}=\frac{\rho k}{\mu \omega}$.

The effective diffusivities $\Gamma_{\mathrm{k}}$ and $\Gamma_{\omega}$ are calculated as,

$$
\begin{gathered}
\Gamma_{\mathrm{k}}=\mu+\frac{\mu_{\mathrm{t}}}{\operatorname{Pr}_{\mathrm{k}}} \\
\Gamma_{\omega}=\mu+\frac{\mu_{\mathrm{t}}}{\operatorname{Pr}_{\omega}}
\end{gathered}
$$

Here, the turbulent viscosity is computing as follows,

$$
\mu_{\mathrm{t}}=\alpha^{*} \frac{\rho \mathrm{k}}{\omega}
$$


The dissipation equations for $\mathrm{k}$ and $\omega$ are given by,

$$
\begin{array}{r}
Y_{k}=\rho \beta^{*} f_{\beta^{*}} k \omega \\
Y_{\omega}=\rho \beta f_{\beta} \omega^{2}
\end{array}
$$

where the functions $f_{\beta^{*}}$ and $f_{\beta}$ can be seen in details on Fluent User Guide Manual.

As the $\mathrm{k}-\omega$ model has been modified over the years, production terms were added to both the $k$ and $\omega$ equations, which improved the accuracy of the model for predicting free shear flows by combination of $\omega$ and $\varepsilon$ equations. The idea of this new turbulence model is to retain the accurate formulation of the $\mathrm{k}-\omega$ model in the boundary-layer region and to that add the advantage of the $\mathrm{k}-\varepsilon$ model in the region of free shear layers to reduce the sensitivity to the free stream flow. To achieve this idea the $\mathrm{k}-\varepsilon$ model is transformed into an equivalent $\mathrm{k}-\omega$ formulation. This new method is called Shear-Stress Transport (SST) $\mathrm{k}-\omega$ turbulence model.

Shear-Stress Transport (SST) k-omega model was proposed as a type of hybrid model, combining two models in order to better calculate flow in the near-wall region. It was designed in response to the problem of the k-epsilon model unsatisfactory near-wall performance for boundary layers with adverse pressure gradients. It utilizes a standard k-epsilon model to calculate flow properties in the free-stream (turbulent) flow region far from the wall, while using a modified k-epsilon model near the wall using the turbulence frequency $\omega$ as a second variable instead of turbulent kinetic energy dissipation term $\varepsilon$. Since the air in this project is flowing between two flat fins very close to each other, is expected that the boundary layer flow has a strong influence on the results, and properly modeling this near-wall flow could be important for accuracy of the calculations.

Shear-Stress Transport (SST) k-omega model was developed by Menter (1994). The SST k-omega model is similar to the standard k-omega model, but includes the following refinements, Ansys Fluent (2010): 
- The standard k-omega model and the transformed k-epsilon model are both multiplied by a blending function and both models are added together. The blending function is designed to be one in the near-wall region, which activates the standard k-omega model, and zero away from the surface, which activates the transformed k-epsilon model.

- The SST model incorporates a damped cross-diffusion derivative term in the $\omega$ equation.

- The definition of the turbulent viscosity is modified to account for the transport of the turbulent shear stress.

- The modeling constants are different.

These features make the SST k-omega model more accurate and reliable for a wider class of flows (eg, adverse pressure gradient flows, airfoils, transonic shock waves) than the standard k-omega model. Low Reynolds number modifications were proposed by Wilcox (1998) for the $\mathrm{k}$ model. It is important to note that all k-omega models can be integrated through the viscous sublayer. The terms were mainly added to reproduce the peak in the turbulence kinetic energy observed in DNS data very close to the wall.

The form of the SST k-omega turbulence model is very similar to standard k-omega turbulence model as shown on Equation 24 and 25,

$$
\begin{aligned}
& \frac{D k}{D t}=\frac{\partial}{\partial x_{j}}\left[\Gamma_{k} \frac{\partial k}{\partial x_{j}}\right]+\tilde{G}_{k}+Y_{k} \\
& \frac{D \omega}{D t}=\frac{\partial}{\partial x_{j}}\left[\Gamma_{\omega} \frac{\partial \omega}{\partial x_{j}}\right]+G_{\omega}-Y_{\omega}+D_{\omega}
\end{aligned}
$$

where $D_{\omega}$ represents the cross diffusion term. This term is responsible to blend the standard $k-\omega$ model and the standard $k-\varepsilon$ model. To blend both models together, 
the standard $k-\varepsilon$ model must be transformed into equations based on $k$ and $\omega$, which leads to the introduction of a cross diffusion term, calculated as,

$$
\mathrm{D}_{\omega}=2\left(1-\mathrm{F}_{1}\right) \rho \frac{1}{\omega \operatorname{Pr}_{\omega, 2}} \frac{\partial \mathrm{k}}{\partial \mathrm{x}_{\mathrm{j}}} \frac{\partial \omega}{\partial \mathrm{x}_{\mathrm{j}}}
$$

The turbulent viscosity is computed as follows,

$$
\mu_{\mathrm{t}}=\frac{\rho \mathrm{k}}{\omega} \frac{1}{\max \left[\frac{1}{\alpha}, \frac{S F_{2}}{a_{1} \omega}\right]}
$$

where $S$ is the strain rate magnitude and $\alpha^{*}$ is defined by Equation 29.

The effective diffusivities are calculated according to Equations 30 and 31, with the Prandtl values for $\mathrm{k}$ and $\omega$ being computed as,

$$
\begin{aligned}
& \operatorname{Pr}_{k}= \frac{1}{\frac{F_{1}}{\operatorname{Pr}_{k, 1}}+\frac{\left(1-F_{1}\right)}{\operatorname{Pr}_{k, 2}}} \\
& \operatorname{Pr}_{\omega}=\frac{1}{\frac{F_{1}}{\operatorname{Pr}_{\omega, 1}}+\frac{\left(1-F_{1}\right)}{\operatorname{Pr}_{\omega, 2}}}
\end{aligned}
$$

where:

$$
\begin{gathered}
F_{1}=\tanh \left(\varphi_{1}^{4}\right) \\
\varphi_{1}=\min \left[\max \left(\frac{\sqrt{\mathrm{k}}}{0.09 \omega \mathrm{y}}, \frac{500 \mu}{\rho \mathrm{y}^{2} \omega}\right), \frac{4 \rho \mathrm{k}}{\operatorname{Pr}_{\omega, 2} \mathrm{D}_{\omega}^{+} \mathrm{y}^{2}}\right] \\
\mathrm{D}_{\omega}^{+}=\max \left[2 \rho \frac{1}{\operatorname{Pr}_{\omega, 2}} \frac{1}{\omega} \frac{\partial \mathrm{k}}{\partial \mathrm{x}_{\mathrm{j}}} \frac{\partial \omega}{\partial \mathrm{x}_{\mathrm{j}}}, 10^{-10}\right] \\
\mathrm{F}_{2}=\tanh \left(\varphi_{2}^{2}\right)
\end{gathered}
$$




$$
\varphi_{2}=\max \left[2 \frac{\sqrt{\mathrm{k}}}{0.09 \omega \mathrm{y}}, \frac{500 \mu}{\rho \mathrm{y}^{2} \omega}\right]
$$

The generation of $\mathrm{k}$ and $\omega$ are given by,

$$
\begin{aligned}
& \tilde{G}_{k}=\min \left(G_{k}, 10 \rho \beta^{*} k \omega\right) \\
& G_{\omega}=\frac{\alpha}{v_{t}} \tilde{G}_{k}
\end{aligned}
$$

where $G_{k}$ is determined from Equation 26 and $\alpha$ from Equation 28.

\subsubsection{Summary of Turbulence Models}

There were many turbulence models to choose from to model the heat exchanger. After considering the availability of models in Ansys Fluent, the applicability, whether it is computationally economical, and accuracy based on past validation and the current researches, the standard and RNG k-epsilon and SST k-omega models were chosen to carry out the present simulations. The k-epsilon models are a well-known model, and are also computationally economical, while the SST k-omega model is more accurate for boundary layer flow.

Also, the value for turbulent intensity (I), when necessary, was calculated by Equation 48. Anyway, in numerical validation (Appendix A) is presented the impact on turbulence intensity on Colburn and Friction factor for the Reynolds number range considered in this project.

$$
I=0.16 \operatorname{Re}^{-\frac{1}{8}}
$$




\subsection{Near-Wall Treatments for Wall-Bounded Turbulent Flows}

In this section it is presented a brief summary about the near-wall treatments implemented by solver developer. For the purposed of this work, the theory guide provided by Ansys Fluent (2010) is enough, in which is conveniently reproduced here to turn it an easy reference to current reader. All information and credit shown in this section should be given to Ansys Fluent (2010).

Turbulent flows are significantly affected by the presence of walls. Obviously, the mean velocity field is affected through the no-slip condition that has to be satisfied at the wall. However, the turbulence is also changed by the presence of the wall in nontrivial ways. Very close to the wall, viscous damping reduces the tangential velocity fluctuations, while kinematic blocking reduces the normal fluctuations. Toward the outer part of the near-wall region, however, the turbulence is rapidly augmented by the production of turbulence kinetic energy due to the large gradients in mean velocity. The near-wall modeling significantly impacts the fidelity of numerical solutions, since the walls are the main source of mean vorticity and turbulence. Also, near-wall region that the solution variables have large gradients, and the momentum and other scalar transports occur most vigorously. Therefore, accurate representation of the flow in the near-wall region determines successful predictions of wall-bounded turbulent flows.

Numerous experiments have shown that the near-wall region can be largely subdivided into three layers. In the innermost layer, called the "viscous sublayer", the flow is almost laminar, and the (molecular) viscosity plays a dominant role in momentum and heat or mass transfer. In the outer layer, called the fully-turbulent layer, turbulence plays a major role. Finally, there is an interim region between the viscous sublayer and the fully turbulent layer, where the effects of molecular viscosity and turbulence are equally important. Figure 37 illustrates these subdivisions of the near-wall region, plotted in semi-log coordinates. 


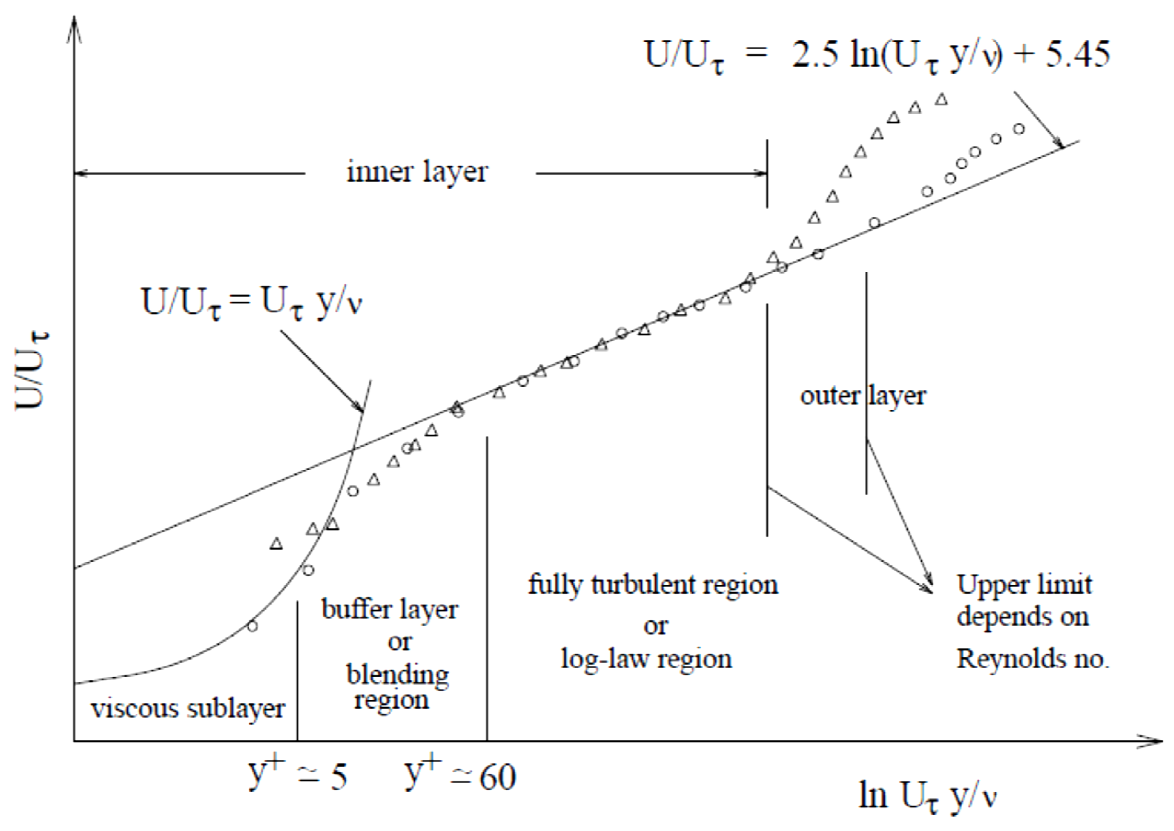

Figure 37 - Subdivisions of the Near-Wall Region.

For y-plus $\left(\mathrm{y}^{+}\right)$defined by Equation 49:

$$
\mathrm{y}^{+} \equiv \frac{\rho \mathrm{u}_{\tau} \mathrm{y}}{\mu}
$$

where the friction velocity is calculated in Equation 50.

$$
u_{\tau}=\sqrt{\frac{\tau_{w}}{\rho}}
$$

Traditionally, there are two approaches to modeling the near-wall region. In one approach, the viscosity-affected inner region (viscous sublayer and buffer layer) is not resolved. Instead, semi-empirical formulas called "wall functions" are used to bridge the viscosity-affected region between the wall and the fully-turbulent region. The use of wall functions obviates the need to modify the turbulence models to account for the presence of the wall.

In another approach, the turbulence models are modified to enable the viscosityaffected region to be resolved with a mesh all the way to the wall, including the 
viscous sublayer. For the purposes of discussion, this will be termed the "near-wall modeling" approach. These two approaches are depicted schematically in Figure 38.

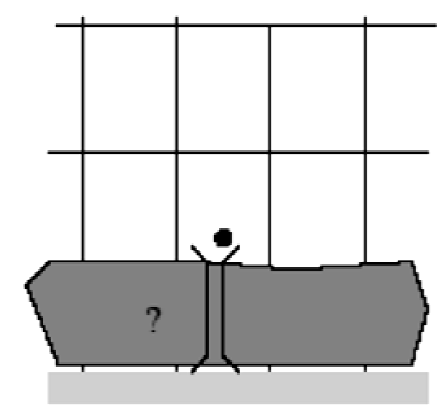

Wall Function Approach

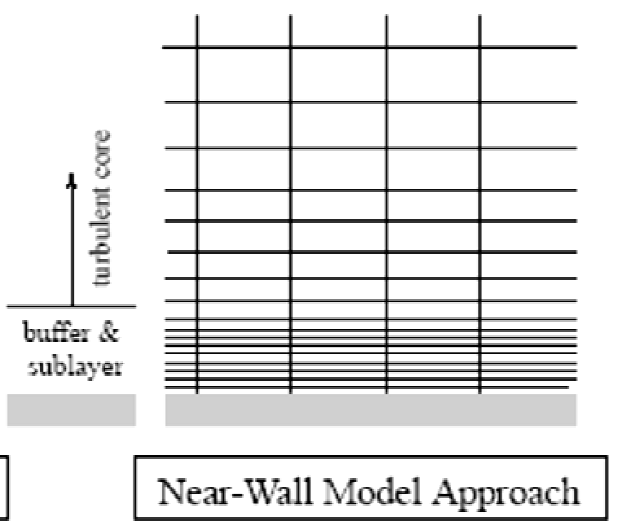

Near-Wall Model Approach

Figure 38 - Two approaches to Near-Wall Treatments in Ansys Fluent (2010).

Wall functions are a set of semi-empirical formulas and functions that in effect "bridge" or "link" the solution variables at the near-wall cells and the corresponding quantities on the wall. The wall functions comprise:

- Laws-of-the-wall for the mean velocity and temperature (or other scalars).

- Formulae for the near-wall turbulent quantities.

In Ansys Fluent, depending on the choice of turbulent model, are offers three choices of wall-function approaches:

- Standard Wall Functions

- Non-Equilibrium Wall Functions

- Enhanced Wall Functions (as a part of EWT - Enhanced Wall Treatment)

The standard wall functions implemented in Ansys Fluent are based on the work of Launder and Spalding (1974), and have been most widely used in industrial flows. In addition to the standard wall function, a two layer-based, non-equilibrium wall function, Kim and Choudhury (1995) is also available. The key elements in the nonequilibrium wall functions are: 
- Launder and Spalding's log-law for mean velocity is sensitized to pressuregradient effects.

- The two-layer-based concept is adopted to compute the budget of turbulence kinetic energy in the wall-neighboring cells.

Because of the capability to partly account for the effects of pressure gradients, the non-equilibrium wall functions are recommended for use in complex flows involving separation, reattachment, and impingement, where the mean flow and turbulence are subjected to pressure gradients and rapid changes. In such flows, improvements can be obtained, particularly in the prediction of wall shear (skin-friction coefficient) and heat transfer (Nusselt or Stanton number).

The standard wall functions give reasonable predictions for the majority of highReynolds-number wall-bounded flows. The non-equilibrium wall functions further extend the applicability of the wall function approach by including the effects of pressure gradient; however, the above wall functions become less reliable when the flow conditions depart too much from the ideal conditions underlying the wall functions. Examples are:

- Pervasive low-Reynolds-number or near-wall effects (eg, flow through a small gap or highly viscous, low-velocity fluid flow).

- Massive transpiration through the wall (blowing/suction).

- Severe pressure gradients leading to boundary layer separations.

- Strong body forces (eg, flow near rotating disks, buoyancy-driven flows).

- High three-dimensionality in the near-wall region.

In compact heat exchanger, at least two above listed features are considered critically important and in this case non-equilibrium wall function fails. Thus, it was necessary to employ the near-wall modeling approach, combined with the adequate mesh resolution in the near-wall region. Ansys Fluent provides the enhanced wall 
treatment for such situations. This approach can be used with the k-epsilon models and is default for SST k-omega model.

Enhanced Wall Treatment for the $\varepsilon$-equation is a near-wall modeling method that combines a two-layer model with so-called enhanced wall functions. If the near-wall mesh is fine enough to be able to resolve the viscous sublayer (typically with the first near-wall node placed at $\mathrm{y}^{+} \approx 1$ ), then the enhanced wall treatment will be identical to the traditional two-layer zonal model. However, the restriction that the near-wall meshes must be sufficiently fine everywhere might impose a too large computational requirement. Ideally, one would like to have a near-wall formulation that could be used with coarse meshes (usually referred to as wall-function meshes), as well as fine meshes (low-Reynolds-number meshes). In addition, excessive error should not be incurred for the intermediate meshes, which the first near-wall node is placed neither in the fully turbulent region and the wall functions are suitable, nor in the direct vicinity of the wall at $\mathrm{y}^{+} \approx 1$, where the low-Reynolds number approach is adequate.

To achieve the goal of having a near-wall modeling approach that will possess the accuracy of the standard two-layer approach for fine near-wall meshes and that, at the same time, will not significantly reduce accuracy for wall-function meshes, Ansys Fluent combines the two-layer model with enhanced wall functions, where the viscosity-affected near-wall region is completely resolved all the way to the viscous sublayer. The two-layer approach is an integral part of the enhanced wall treatment and is used to specify both epsilon and the turbulent viscosity in the near-wall cells. In this approach, the whole domain is subdivided into a viscosity-affected region and a fully-turbulent region.

Unlike the standard $\varepsilon$-equation, the $\omega$-equation can be integrated through the viscous sublayer, without the need for a two-layer approach. This formulation is the default for all $\omega$-equation based models in Ansys Fluent. 


\subsection{Pressure-Velocity Coupling}

Ansys Fluent (2010) provides some options to choose among different pressurevelocity coupling algorithms, such as: SIMPLE, SIMPLEC and COUPLED. The pressure-based solver allows solving flow in either a segregated or coupled manner. All the aforementioned schemes, except the "COUPLED" scheme, are based on the predictor-corrector approach and segregated algorithm. The main reason to use a coupled method instead of segregated approach was the convergence speed. The software developer provides the comparison between these methodologies on validation case (RAE 2822 airfoil) and the summary results is shown in Table 4.

Table 4 - Comparison of computer resources and solver performance for the RAE 2822 airfoil.

\begin{tabular}{ccccc} 
Solver & Memory (MB) & $\begin{array}{c}\text { Time per Iteration } \\
\text { (seconds) }\end{array}$ & $\begin{array}{c}\text { Iterations to } \\
\text { Convergence }\end{array}$ & $\begin{array}{c}\text { Time to } \\
\text { Convergence } \\
\text { (hours) }\end{array}$ \\
\hline Segregated & 172 & 2.10 & 2570 & 1.50 \\
Coupled & 259 & 3.16 & 298 & 0.27 \\
\hline
\end{tabular}

Table 4 presents the solver performance and resource requirements for each algorithm. As expected, the segregated solver uses the least memory. However, because of the close coupling of the momentum and continuity equations for this case, the data shows that the segregated solver required 2,570 iterations to reach convergence. In contrast, the pressure based-coupled solver required only 298 iterations to converge.

Besides the convergence results reported by software developer and shown in Table 4 , in the present work, preliminary results showed that coupled algorithm converged faster than segregated method (SIMPLE family). And, considering that in an optimization process many cases are necessary to reach a good quality RSM or direct optimization, this algorithm was chosen in this thesis. In open literature, there is no related paper that applied this algorithm.

The SIMPLE (Semi-Implicit Method for Pressure-Linked Equations) algorithm was devised by Patankar and Spalding, beginning with Spalding (1977) and followed by 
Patankar (1980). Various forms probably are the most widely used of primitivevariable methods and are typically the core algorithm in most commercial engineering CFD codes. The method is very robust on coarse grids, but exhibits quite low asymptotic convergence rates. Hence, it is effective for calculating rough solutions to even very complex problems, but rapidly loses its effectiveness as grids are refined to achieve more spatial accuracy. Through the years numerous modifications were observed to the basic SIMPLE procedure, specifically intended to improve performance for time-dependent problems, such as SIMPLEC (SIMPLEConsistent).

The SIMPLE algorithm uses a relationship between velocity and pressure corrections to enforce mass conservation and to obtain the pressure field. SIMPLE is the most applied, but many problems will benefit from the use of SIMPLEC. For relatively uncomplicated problems (laminar flows with no additional models activated), in which convergence is limited by the pressure-velocity coupling, is often obtained a converged solution more quickly using SIMPLEC. For complicated flows involving turbulence and/or additional physical models, SIMPLEC will improve convergence only if it was limited by the pressure-velocity coupling. Often, it will be one of the additional modeling parameters that limit convergence; in this case, SIMPLE and SIMPLEC will give similar convergence rates, Ansys Fluent (2010).

SIMPLE algorithm is widely used in numerical simulation in compact heat exchanger, Song and Wang (2013), Hu et al. (2013), Min et al. (2012). SIMPLEC algorithm has been used less than SIMPLE algorithm, Herpe et al. (2009) and Wu and Tao (2007).

The SIMPLE algorithm gives a method of calculating pressure and velocities. The method is iterative, and when other scalars are coupled to the momentum equations the calculation needs to be done sequentially. The sequence of operations in a CFD procedure, which employs the SIMPLE algorithm, is given in Figure 39. 


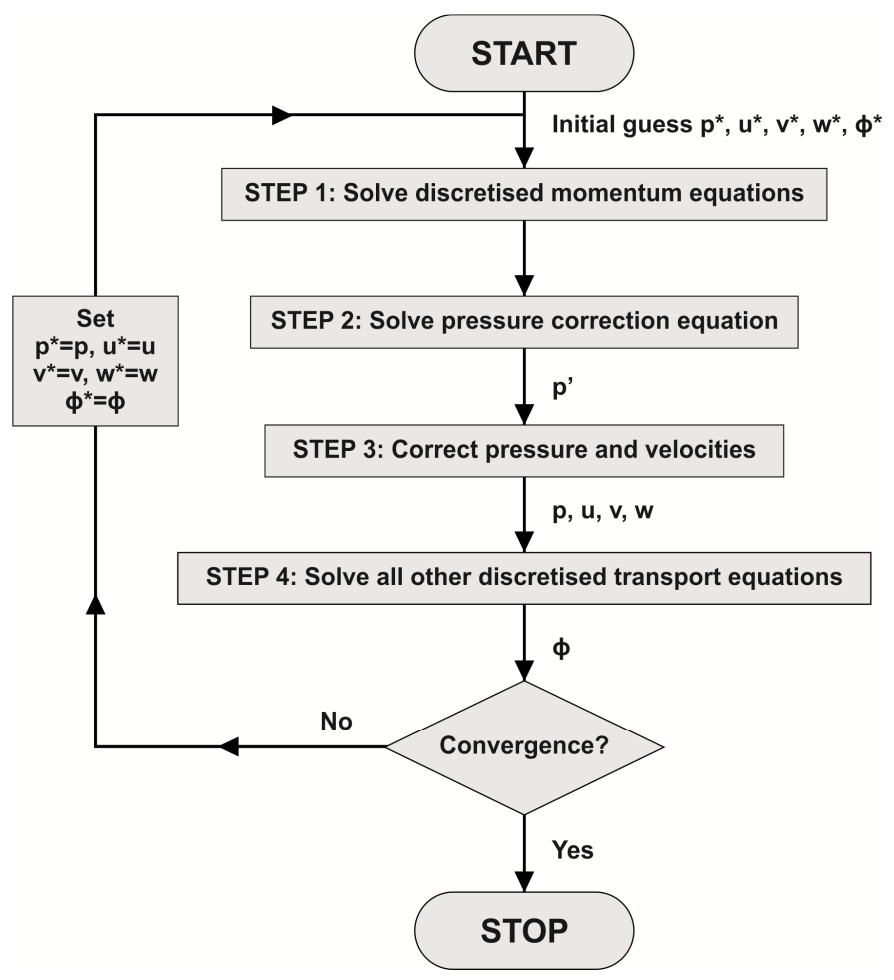

Figure 39 - Sequence of operations in SIMPLE algorithm, Ansys Fluent (2010).

SIMPLEC algorithm of Vandoormaal and Raithby (1984) follows the same steps as the SIMPLE algorithm, with the difference that the momentum equations are manipulated so that the SIMPLEC velocity correction equations omit terms that are less significant than those in SIMPLE. The sequence of operations of SIMPLEC is identical to that of SIMPLE, Figure 39.

Coupled scheme obtains a robust and efficient single phase implementation for steady-state flows. The pressure-based segregated algorithm solves the momentum equation and pressure correction equations separately. This semi-implicit solution method results in slow convergence. The coupled algorithm solves the momentum and pressure-based continuity equations together. The full implicit coupling is achieved through an implicit discretization of pressure gradient terms in the momentum equations, and an implicit discretization of the face mass flux, including the Rhie-Chow pressure dissipation terms. Obviously, Ansys Fluent (2010) carry out the implementation features of this coupled scheme, however the Tao et al. (2005) leads an enlightening work comparing the convergence between coupled and segregated scheme (SIMPLE family), applied in the study of heat transfer 
enhancement. The discretization and solution procedure of coupled scheme is well given in this paper and this is not present here, since this discussion is not the core of this present work.

\subsection{Spatial Discretization Scheme}

Maliska (2004) and Versteeg and Malalasekera (2007) provide an excellent textbooks about this topic and this is not reviewed again. In Fluent environment, when first-order accuracy is chosen, quantities at cell faces are determined by assuming that the cell-center values of any field variable represent a cell-average value and hold throughout the entire cell; the face quantities are identical to the cell quantities. Thus, when first-order upwind is selected, the face value is set equal to the cell-center value of the upstream cell. When second-order accuracy is desired, quantities at cell faces are computed using a multidimensional linear reconstruction approach, Barth and Jespersen (1989). In this approach, higher-order accuracy is achieved at cell faces through a Taylor series expansion of the cell-centered solution about the cell centroid. By practical way, the guide line provide by Ansys Fluent (2010) suggest that when the flow is aligned with the mesh (eg, laminar flow in a rectangular duct modeled with a quadrilateral or hexahedral mesh), the first-order upwind discretization may be acceptable. When the flow is not aligned with the mesh (ie, when it crosses the mesh lines obliquely), the first-order convective discretization increases the numerical discretization error (numerical diffusion). For triangular and tetrahedral meshes, since the flow is never aligned with the mesh, generally is obtained more accurate results by using the second-order discretization. For quad/hex meshes, better results are also obtained using the second-order discretization, especially for complex flows. In summary, while the first-order discretization generally yields better convergence than the second-order scheme, it generally yields less accurate results, especially on tri/tetra meshes. For most cases, is possible to use the second-order scheme from the start of the calculation. However, in some cases, it is needed to start with the first-order scheme and then switch to the second-order scheme after a few iterations. This strategy was adopted in this thesis. 


\subsubsection{Taylor series expansion}

Discretization of the convective term requires the approximation of the first derivative, $\partial(\rho u \phi) / \partial \mathbf{x}$. Thus, considering any continuous differentiable function $\phi_{\mathrm{x}}$ can, in the vicinity of $x_{i}$, be expressed as a Taylor series:

$$
\phi(x)=\phi\left(x_{i}\right)+\left(x-x_{i}\right)\left(\frac{\partial \phi}{\partial x}\right)_{i}+\frac{\left(x-x_{i}\right)^{2}}{2 !}\left(\frac{\partial^{2} \phi}{\partial x^{2}}\right)_{i}+\frac{\left(x-x_{i}\right)^{3}}{3 !}\left(\frac{\partial^{3} \phi}{\partial x^{3}}\right)_{i}+\cdots+\frac{\left(x-x_{i}\right)^{n}}{n !}\left(\frac{\partial^{n} \phi}{\partial x^{n}}\right)_{i}+H
$$

where $\mathrm{H}$ means "higher order terms". By replacing $\mathrm{x}$ by $\mathrm{x}_{\mathrm{i}+1}$ or $\mathrm{x}_{\mathrm{i}-1}$ in this equation, one obtains expressions for the variable values at these points in terms of the variable and its derivatives at $x_{i}$. This can be extended to any other point near $x_{i}$, for example, $\mathrm{x}_{\mathrm{i}+2}$ and $\mathrm{x}_{\mathrm{i}-2}$.

Using these expansions, one can obtain approximate expressions for the first and higher derivatives at point $x_{i}$ in terms of the function values at neighboring points. For example, using Equation 51 for $\phi$ at $x_{i+1}$,, is shown that:

$$
\left(\frac{\partial \phi}{\partial x}\right)_{i}=\frac{\phi_{i+1}-\phi_{i}}{x_{i+1}-x_{i}}-\frac{x_{i+1}-x_{i}}{2}\left(\frac{\partial^{2} \phi}{\partial x^{2}}\right)_{i}-\frac{\left(x_{i+1}-x_{i}\right)^{2}}{6}\left(\frac{\partial^{3} \phi}{\partial x^{3}}\right)_{i}+H
$$

Another expression may be derived using the series Equation 51 at $\mathrm{x}_{\mathrm{i}-1}$ :

$$
\left(\frac{\partial \phi}{\partial x}\right)_{i}=\frac{\phi_{i}-\phi_{i-1}}{x_{i}-x_{i-1}}+\frac{x_{i}-x_{i-1}}{2}\left(\frac{\partial^{2} \phi}{\partial x^{2}}\right)_{i}-\frac{\left(x_{i}-x_{i-1}\right)^{2}}{6}\left(\frac{\partial^{3} \phi}{\partial x^{3}}\right)_{i}+H
$$

Also, another expression may be obtained by using Equation 51 at both $\mathrm{x}_{\mathrm{i}-1}$ and $\mathrm{x}_{\mathrm{i}+1}$ : 


$$
\left(\frac{\partial \phi}{\partial x}\right)_{i}=\frac{\phi_{i+1}-\phi_{i-1}}{x_{i+1}-x_{i-1}}-\frac{\left(x_{i+1}-x_{i}\right)^{2}-\left(x_{i}-x_{i-1}\right)^{2}}{2\left(x_{i+1}-x_{i-1}\right)}\left(\frac{\partial^{2} \phi}{\partial x^{2}}\right)_{i}-\frac{\left(x_{i+1}-x_{i}\right)^{3}+\left(x_{i}-x_{i-1}\right)^{3}}{6\left(x_{i+1}-x_{i-1}\right)}\left(\frac{\partial^{3} \phi}{\partial x^{3}}\right)_{i}+H
$$

All three expressions are exact if all terms on the right hand side are retained. Because the higher-order derivatives are unknown, these expressions are not of great value as they stand. However, if the distance between the grid points, ie, $x_{i}-x_{i-1}$ and $x_{i}-x_{i+1}$ is small, the higher-order terms will be small except in the unusual situation, in which the higher derivatives are locally very large. Ignoring the latter possibility, approximations to the first derivative result from truncating each of the series after the first terms on the right hand sides:

$$
\begin{aligned}
& \left(\frac{\partial \phi}{\partial \mathrm{x}}\right)_{\mathrm{i}} \approx \frac{\phi_{\mathrm{i}+1}-\phi_{\mathrm{i}}}{\mathrm{x}_{\mathrm{i}+1}-\mathrm{x}_{\mathrm{i}}}+\mathrm{O}(\Delta \mathrm{x}) \\
& \left(\frac{\partial \phi}{\partial \mathrm{x}}\right)_{\mathrm{i}} \approx \frac{\phi_{\mathrm{i}}-\phi_{\mathrm{i}-1}}{\mathrm{x}_{\mathrm{i}}-\mathrm{x}_{\mathrm{i}-1}}+\mathrm{O}(\Delta \mathrm{x}) \\
& \left(\frac{\partial \phi}{\partial \mathrm{x}}\right)_{\mathrm{i}} \approx \frac{\phi_{\mathrm{i}+1}-\phi_{\mathrm{i}-1}}{\mathrm{x}_{\mathrm{i}+1}-\mathrm{x}_{\mathrm{i}-1}}+\mathrm{O}\left(\Delta \mathrm{x}^{2}\right)
\end{aligned}
$$

These are the forward (FDS), backward (BDS), and central-difference (CDS) schemes mentioned earlier, respectively. The terms that were deleted from the right hand sides are called the truncation errors; they measure the accuracy of the approximation and determine the rate at which the error decreases as the spacing between points is reduced. In particular, the first truncated term is usually the principal source of error.

Second derivatives appear in the diffusive terms. To estimate the second derivative at a point, one may use the approximation for the first derivative twice. This is the only approach possible when the fluid properties are variable, it since is needed the derivative of the product of diffusion coefficient and the first derivative. For example, by inserting approximations for the first derivatives at locations $x_{i+1}$ and $x_{i}$, an approximation for the second derivative is obtained: 


$$
\left(\frac{\partial^{2} \phi}{\partial x^{2}}\right)_{i} \approx \frac{\left(\frac{\partial \phi}{\partial x}\right)_{i+1}-\left(\frac{\partial \phi}{\partial x}\right)_{i}}{x_{i+1}-x_{i}}+O\left(\Delta x^{2}\right)
$$

\subsubsection{Upwind interpolation (UDS)}

Figure 40 shows a typical control volume for a Cartesian 2D grid, which is applied to exemplify the upwind interpolation method.

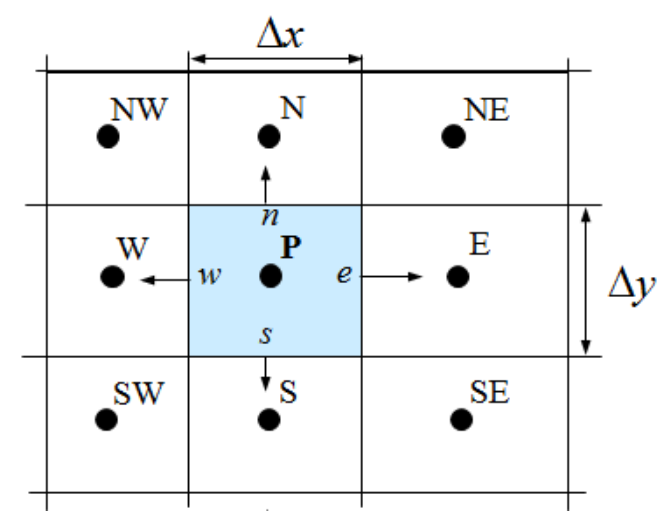

Figure 40 - Typical control volume and the notation used for a Cartesian 2D grid.

Approximating $\phi_{\mathrm{e}}$, for its value at the node upstream of " $\mathrm{e}$ " is equivalent to using a backward or forward difference approximation for the first derivative (depending on the flow direction), hence the name upwind differencing scheme (UDS) for this approximation. In UDS, $\phi_{\mathrm{e}}$ is approximated as:

$$
\phi_{e}=\left\{\begin{array}{l}
\phi_{P} \text { if }\left(v_{e}>0\right) \\
\phi_{E} \text { if }\left(v_{e}<0\right)
\end{array}\right.
$$

Applying Taylor series expansion about $\mathrm{P}$ gives (for Cartesian grid and $\mathrm{v}_{\mathrm{e}}>0$ ): 


$$
\phi_{e}=\phi_{P}+\left(x_{e}-x_{P}\right)\left(\frac{\partial \phi}{\partial x}\right)_{P}+\frac{\left(x_{e}-x_{P}\right)^{2}}{2}\left(\frac{\partial^{2} \phi}{\partial x^{2}}\right)_{P}
$$

Overall, considering the previous sections and these assessments, the setup evaluated in Ansys Fluent could be summarized in Table 5:

Table 5 - Summary of the setup evaluated in FLUENT.

\begin{tabular}{cccc}
\hline $\begin{array}{c}\text { Turbulence } \\
\text { model }\end{array}$ & $\begin{array}{c}\text { Near-Wall } \\
\text { Treatments }\end{array}$ & $\begin{array}{c}\text { Pressure-Velocity } \\
\text { Coupling }\end{array}$ & $\begin{array}{c}\text { Spatial } \\
\text { Discretization }\end{array}$ \\
\hline $\begin{array}{c}\text { Standard k-epsilon } \\
\text { RNG k-epsilon } \\
\text { SST k-omega }\end{array}$ & Enhanced & COUPLED & $\begin{array}{c}\text { Second order } \\
\text { Upwind }\end{array}$ \\
\hline
\end{tabular}

In general, numerical setup outlined is routinely used by scientific community for many years, which study augmentation heat transfer in compact heat exchanger. Since the major phenomenon in augmentation heat transfer, already discussed in Chapter 1 and 2, are known as contained in the large-scale structures, this numerical setup are enough for the purposes of this thesis. In order to confirm this assessment, Bhutta et al. (2012) provide a literature review focused on the applications of Computational Fluid Dynamics (CFD) in the field of heat exchanger. They also evaluated the numerical setup applied on this thesis, which concluded that CFD is an effective tool for predicting the behavior and performance of a wide variety of heat exchangers, where compact heat exchanger is within.

Following, the next sections present the computational domain and boundary conditions. Appendix A show the validation results of each configuration, according to Table 5, and the conclusion about the final setup applied to meta-modeling and optimization process. 


\subsection{Computational Domain and Boundary Conditions}

A diagram of the studied model is shown in Figure 41, which consists of the air flow area between two fins of plain-fin with two rows of tubes, and a schematic model is shown in Figure 42, according to geometrical values listed in Table 6. Computational domain was extended upstream 1 time of the original heat transfer zone (main domain) to ensure the uniform velocity distribution at the inlet, and 5 times downstream to allow the use of outflow boundary condition. The convergence criterion was to residuals less than $10^{-7}$ for the flow equations and $10^{-9}$ for the energy equation.

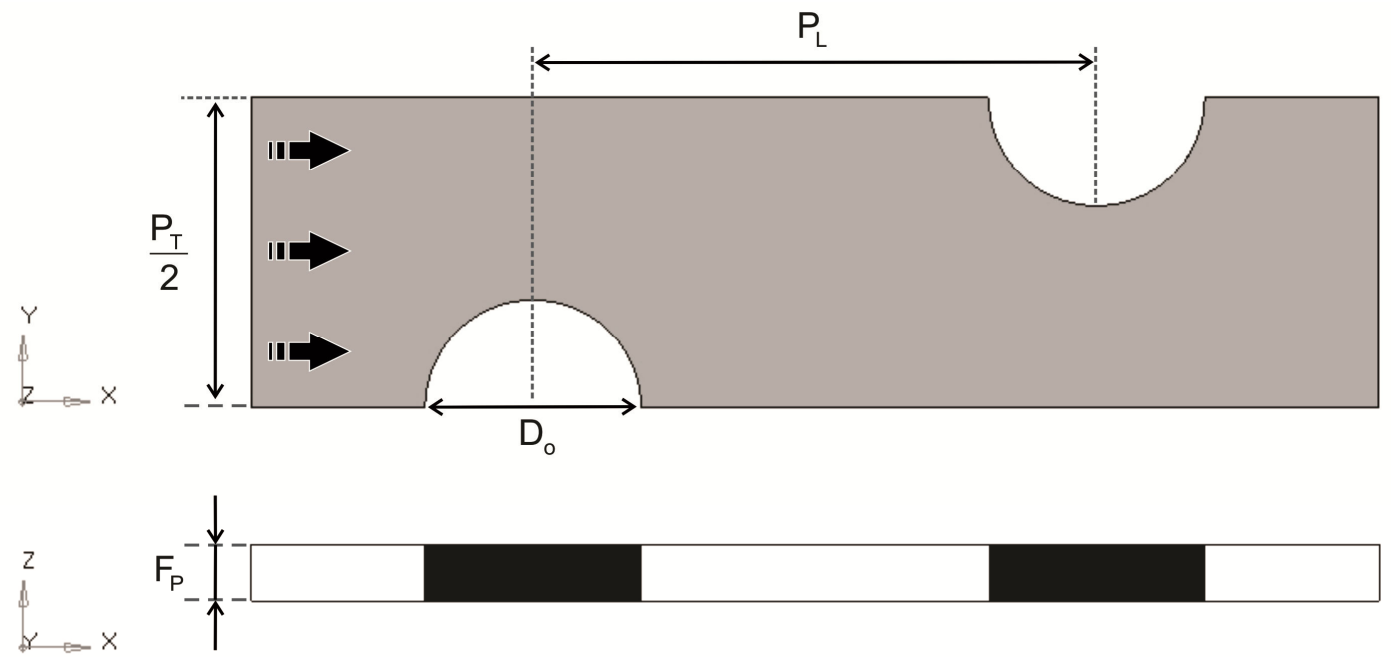

Figure 41 - Illustration of the main computational domain and geometric parameters of the heat exchanger model studied.

Table 6 - Geometric dimensions of heat exchanger model.

\begin{tabular}{lcc}
\hline Fin thickness & $\delta_{\mathrm{f}}$ & $0.08 \mathrm{~mm}$ \\
Fin pitch & $\mathrm{F}_{\mathrm{P}}$ & $2.00 \mathrm{~mm}$ \\
Tube diameter & $\mathrm{D}_{\mathrm{o}}$ & $7.50 \mathrm{~mm}$ \\
Fin collar outside diameter & $\mathrm{D}_{\mathrm{c}}=\mathrm{D}_{\mathrm{o}}+\left(2 \delta_{\mathrm{f}}\right)$ & $7.66 \mathrm{~mm}$ \\
Transverse pitch & $\mathrm{P}_{\mathrm{T}}$ & $22 \mathrm{~mm}$ \\
Longitudinal Pitch & $\mathrm{P}_{\mathrm{L}}$ & $20 \mathrm{~mm}$ \\
Depth of the CHE & $\mathrm{L}=2 . \mathrm{P}_{\mathrm{L}}$ & $40 \mathrm{~mm}$ \\
Number of tube rows & $\mathrm{N}$ & 2 \\
\hline
\end{tabular}


The boundary conditions are described as follows:

\section{Inlet (Dirichlet boundary condition):}

$\mathrm{u}=\mathrm{u}_{\text {inlet }}=\mathrm{cte} ; \mathrm{v}=\mathrm{w}=0 ; \mathrm{T}=\mathrm{T}_{\text {inlet }}=\mathrm{cte} ; \mathrm{I}\left(\right.$ Equation 48) and $\mathrm{D}_{\mathrm{h}}$.

\section{Upstream/Downstream-extended region:}

$$
\begin{aligned}
& x-y \text { plane } \rightarrow \frac{\partial u}{\partial z}=\frac{\partial v}{\partial z}=0 ; w=0 ; \frac{\partial T}{\partial z}=0 \\
& x-z \text { plane } \rightarrow \frac{\partial u}{\partial y}=\frac{\partial w}{\partial y}=0 ; v=0 ; \frac{\partial T}{\partial y}=0
\end{aligned}
$$

\section{Fin and tube (Dirichlet boundary condition):}

$\mathrm{u}=\mathrm{v}=\mathrm{w}=0 ; \mathrm{T}=\mathrm{T}_{\text {wall }}=\mathrm{cte}$

\section{Side plane:}

$$
\frac{\partial u}{\partial y}=\frac{\partial w}{\partial y}=0 ; v=0 ; \frac{\partial T}{\partial y}=0
$$

\section{Outlet (Neumann boundary condition):}

$\frac{\partial \mathrm{u}}{\partial \mathrm{x}}=\frac{\partial \mathrm{v}}{\partial \mathrm{x}}=\frac{\partial \mathrm{w}}{\partial \mathrm{x}}=\frac{\partial \mathrm{T}}{\partial \mathrm{x}}=0$

According to Bardina et al. (1997), turbulence boundary conditions are:

$\mathrm{k}-\varepsilon$

At no-slip surfaces are given by,

$\mathrm{k}=0$ and $\varepsilon=0$

And, zero-gradient conditions are applied at symmetry boundaries.

$\mathrm{k}-\omega$

At no-slip surfaces are given by, 
$\mathrm{k}=0$ and $\omega=10 \frac{6 \mu}{\beta \rho\left(\mathrm{y}_{1}\right)^{2}}$

Where $\mathrm{y}_{1}$ is the distance of the first point away from the wall and $y_{1}^{+} \approx 1$. Zerogradient conditions are applied at symmetry boundaries and $\beta=3 / 40$.

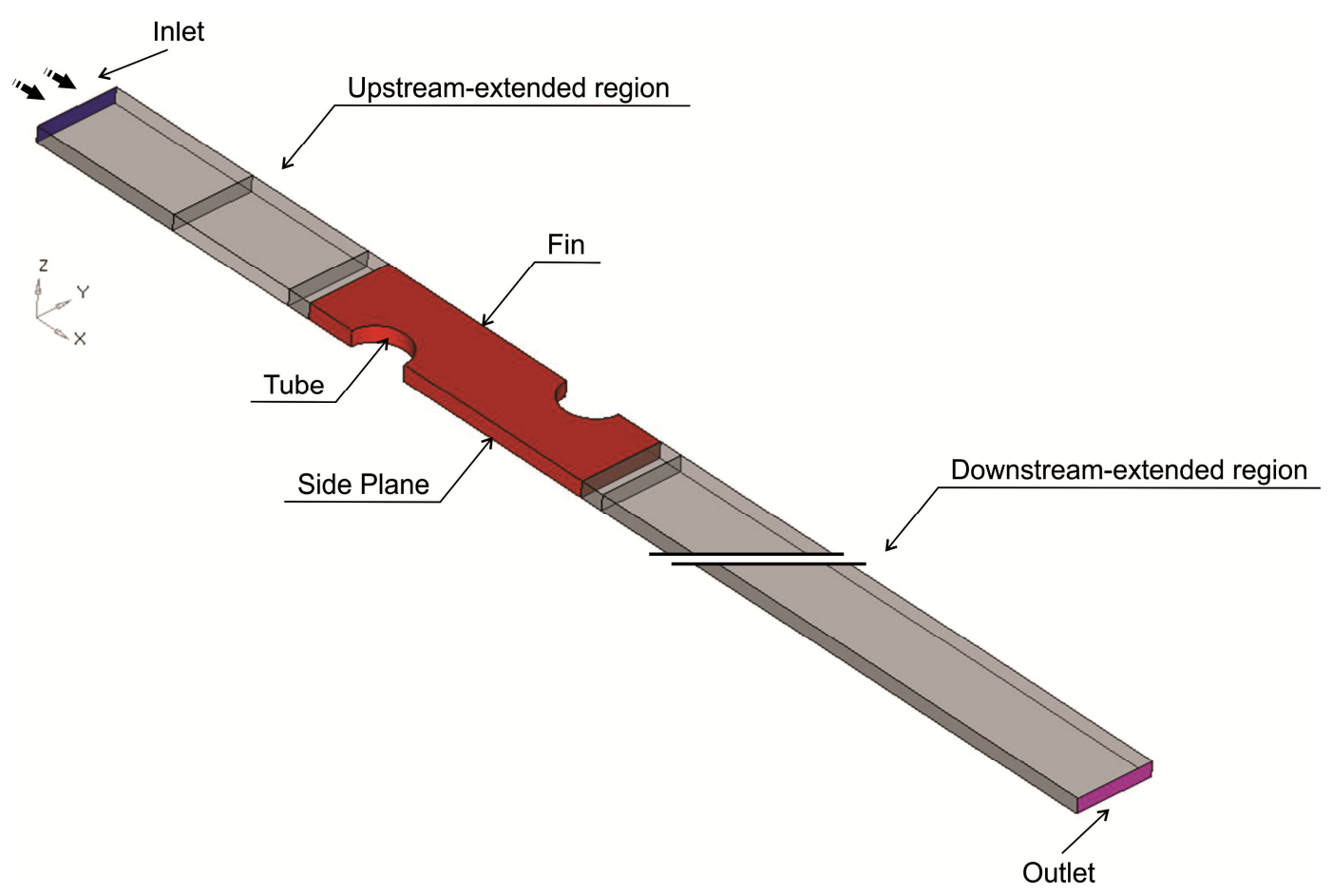

Figure 42 - Computational domain including main domain and extended region.

\subsubsection{Parameter definition}

In compact heat exchanger, the performance parameters depend on the geometry and flow conditions. The flow condition can be characterized by Reynolds number, based here on fin pitch, according to Torii et al. (2002). However, the Reynolds number based on the collar outside diameter tube was applied for validation step, according to correlations proposed by Wang et al. (2000). Colburn factor (Equation 62) and Friction factor (Equation 63) were used to describe the heat transfer and pressure drop of heat exchangers. Thus, these dimensionless parameters are defined as follows: 


$$
\begin{aligned}
& \operatorname{Re}=\frac{\rho u_{c} D}{\mu} \\
& j=\frac{h}{\rho u_{c} c_{p}} \operatorname{Pr}^{\frac{2}{3}} \\
& f=\frac{2 \Delta p}{\rho u_{c}^{2}} \frac{A_{c}}{A_{o}}
\end{aligned}
$$

Where:

$u_{c} \rightarrow$ Velocity at minimum flow area $(\mathrm{m} / \mathrm{s})$

$\mathrm{h} \rightarrow$ Heat transfer coefficient $\left(\mathrm{W} / \mathrm{m}^{2} . \mathrm{K}\right)$

$\mathrm{Pr} \rightarrow$ Prandtl number.

$A_{c} \rightarrow$ Minimum flow area.

$A_{o} \rightarrow$ Wet total surface area of the heat exchanger.

Considering Equations 62 and 63, an ordinary parameter to evaluate the augmentation heat transfer with vortex generator is defined by Equation 64:

$$
J F=\frac{j / j_{0}}{f / f_{0}}
$$

where $j_{0}$ and $f_{o}$ are the Colburn and Friction factors of the heat exchanger without vortex generator (fin-tube), respectively.

Also, in Equation 64, JF $>1$ means that the augmentation heat transfer is larger than the pressure loss penalty. Obviously, if $\mathrm{JF}<1$, this also means that VG configuration produces more pressure drop than enhancement heat transfer. 
Another ordinary parameter for analyzing the augmentation heat transfer with vortex generator is shown in Equation 65, Webb (2005). In this case, the heat transfer is a priority.

$$
J F^{\frac{1}{3}}=\frac{j / j_{0}}{\left(f / f_{0}\right)^{\frac{1}{3}}}
$$

Both parameters $\mathrm{JF}$ and $\mathrm{JF}^{\frac{1}{3}}$ were applied during optimization step and analyzed in chapter 5 .

Average temperature, pressure and Nusselt number of a cross-section were defined in Equation 66, respectively.

$$
\begin{gathered}
\bar{T}=\frac{\iint_{A} u T d A}{\iint_{A} u d A} \\
\bar{p}=\frac{\iint_{A} p d A}{\iint_{A} d A}
\end{gathered}
$$

Total heat transfer, pressure drop and log-mean temperature difference were defined in Equation 67, respectively.

$$
\begin{aligned}
& \mathrm{Q}=\dot{\mathrm{m}} \mathrm{c}_{\mathrm{p}}\left(\overline{\mathrm{T}}_{\text {inlet }}-\overline{\mathrm{T}}_{\text {outlet }}\right) \\
& \Delta \mathrm{p}=\left(\overline{\mathrm{p}}_{\text {inlet }}-\overline{\mathrm{p}}_{\text {outlet }}\right) \\
& \Delta \mathrm{T}=\frac{\left(\mathrm{T}_{\text {wall }}-\overline{\mathrm{T}}_{\text {inlet }}\right)-\left(\mathrm{T}_{\text {wall }}-\overline{\mathrm{T}}_{\text {outlet }}\right)}{\ln \left[\left(\mathrm{T}_{\text {wall }}-\overline{\mathrm{T}}_{\text {inlet }}\right) /\left(\mathrm{T}_{\text {wall }}-\overline{\mathrm{T}}_{\text {outlet }}\right)\right]}
\end{aligned}
$$

And finally, the global heat transfer coefficient was defined in Equation 68:

$$
\mathrm{h}=\frac{\mathrm{Q}}{\mathrm{A}_{\mathrm{o}} \Delta \mathrm{T}}
$$




\section{Meta-modeling and Optimization}

The ever-increasing demand on engineers to lower production costs to withstand competition has prompted engineers to look for rigorous methods of decision making, such as optimization methods, to design and produce products both economically and efficiently. Optimization techniques, having reached a degree of maturity, are being used in a wide spectrum of industries, including aerospace, automotive, chemical, electrical, and manufacturing industries. With rapidly advancing computer technology, computers are becoming more powerful, and correspondingly, the size and the complexity of the problems being solved using optimization techniques are also increasing. Optimization methods, coupled with modern tools of computeraided design, are also being used to enhance the creative process of conceptual and detailed design of engineering systems, Rao (1996).

Optimization is the act of obtaining the best result under given circumstances. In design, construction, and maintenance of any engineering system, engineers have to take many technological and managerial decisions at several stages. The ultimate goal of all such decisions is either to minimize the effort required or to maximize the desired benefit. Since the effort required or the benefit desired in any practical situation can be expressed as a function of certain decision variables, optimization can be defined as the process of finding the conditions, which give the maximum or minimum value of a function. There is no single method available for solving all optimization problems efficiently. Hence, a number of optimization methods have been developed for solving different types of optimization problems.

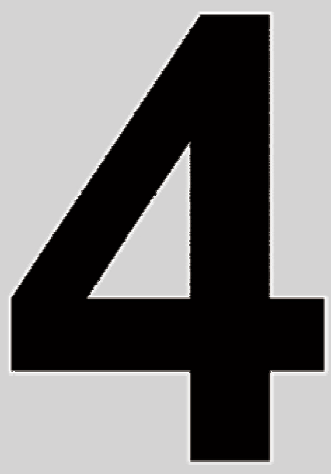


The optimum seeking methods are also known as mathematical programming techniques and are generally studied as a part of operations research. Operations research is a branch of mathematics concerned with the application of scientific methods and techniques to decision making problems and with establishing the best or optimal solutions. Table 7 lists various mathematical programming techniques together with other well-defined areas of operations research. The classification given in Table 7 is not unique.

Table 7 - Methods of operations research, Rao (1996).

\begin{tabular}{lll}
\hline $\begin{array}{l}\text { Mathematical Programming } \\
\text { Technique }\end{array}$ & Stochastic Process Techniques & Statistical Methods \\
\hline Calculus methods & Statistical decision theory & $\begin{array}{l}\text { Regression analysis } \\
\text { Cluster analysis, pattern } \\
\text { recognition }\end{array}$ \\
Calculus of variations & Markov processes & $\begin{array}{l}\text { Design of experiments } \\
\text { Discriminate analysis } \\
\text { (factor analysis) }\end{array}$ \\
Nonlinear programming & Queuing theory & \\
Geometric programming & Renewal theory & \\
Quadratic programming & Simulation methods & \\
Linear programming & Reliability theory & \\
Dynamic programming & & \\
Integer programming & & \\
Stochastic programming & & \\
Separable programming & & \\
Multi-objective programming & & \\
Network methods: CPM and & & \\
PERT & & \\
Game theory & & \\
Simulated annealing & & \\
Genetic algorithms & & \\
Neural Networks & & \\
\hline
\end{tabular}

Mathematical programming techniques are useful in finding the minimum of a function of several variables under a prescribed set of constraints. Stochastic process techniques can be used to analyze problems described by a set of random variables having known probability distributions. Statistical methods enable one to analyze the experimental data and build empirical models to obtain the most accurate representation of the physical situation.

The subject proposed in this thesis is within Mathematical Programming Technique, which is defined as a deterministic problem. Specifically, Genetic Algorithm and Neural Network were applied herein. In the next sections, is presented an overview about these techniques. Before this, is timely to present a general statement of an optimization problem, which is suitable for the present study. 


\subsection{Statement of an optimization problem}

An optimization or a mathematical programming problem can be stated as follows, Rao (1996):

Find $X=\left\{\begin{array}{c}x_{1} \\ x_{2} \\ \vdots \\ x_{n}\end{array}\right\}$ which minimizes $f(X)$

Subject to the constraints,

$$
\begin{array}{ll}
>\mathrm{g}_{\mathrm{j}}(\mathrm{X}) \leq 0, & \mathrm{j}=1,2, \ldots, \mathrm{m} \\
-\mathrm{I}_{\mathrm{j}}(\mathrm{X})=0, & \mathrm{j}=1,2, \ldots, \mathrm{p}
\end{array}
$$

where $X$ is an $n$-dimensional vector called the design vector, $f(X)$ is termed the objective function, and $\mathrm{g}_{\mathrm{j}}(\mathrm{X})$ and $(\mathrm{X})$ and $\mathrm{I}_{\mathrm{j}}(\mathrm{X})$ are known as inequality and equality constraints, respectively. The number of variables $n$ and the number of constraints $m$ and/or $p$ need not be related in anyway. The problem stated outlined is called a constrained optimization problem. Some optimization problems do not involve any constraints and are called as unconstrained optimization problems.

In many practical problems, the design variables cannot be chosen arbitrarily; rather, they have to satisfy certain specified functional and other requirements. The restrictions that must be satisfied to produce an acceptable design are collectively called design constraints. Constraints that represent limitations on the behavior or performance of the system are termed behavior or functional constraints. Constraints that represent physical limitations on design variables, such as availability, manufacturability, and transportability are known as geometric or side constraints.

In general, there will be more than one acceptable design, and the purpose of optimization is to choose the best one of the many acceptable designs available. 
Thus a criterion has to be chosen for comparing the different alternative acceptable designs and for selecting the best one. The criterion with respect to which the design is optimized, when expressed as a function of the design variables, is known as the criterion or merit or objective function. The choice of objective function is governed by the nature of problem and can be one of the most important decisions in the whole optimum design process.

In some situations, there may be more than one criterion to be satisfied simultaneously. An optimization problem involving multiple objective functions is known as a multi-objective programming problem. With multiple objectives there arises a possibility of conflict, and one simple way to handle the problem is to construct an overall objective function as a linear combination of the conflicting multiple objective functions. Thus if $f_{1}(X)$ and $f_{2}(X)$ denote two objective functions, is convenient to construct a new (overall) objective function for optimization as for example, Rao (1996).

$$
f(X)=\alpha_{1} f_{1}(X)+\alpha_{2} f_{2}(X)
$$

where $\alpha_{1}$ and $\alpha_{2}$ are constants whose values indicate the relative importance of one objective function relative to the other.

For the present work, regarding the optimization statement outlined, are evaluated four objective functions which are analyzed separately, where all are considered as single objective. Thus, the optimization statement for the present work is: 
Find $Z=\left\{\begin{array}{c}x_{1} \\ y_{1} \\ \theta_{1} \\ \varphi_{1} \\ x_{2} \\ y_{2} \\ \theta_{2} \\ \varphi_{2}\end{array}\right\}$ which maximize $\mathrm{j}(Z) \rightarrow$ Equation 62

Find $Z=\left\{\begin{array}{c}x_{1} \\ y_{1} \\ \theta_{1} \\ \varphi_{1} \\ x_{2} \\ y_{2} \\ \theta_{2} \\ \varphi_{2}\end{array}\right\}$ which minimize $f(Z) \rightarrow$ Equation 63

Find $Z=\left\{\begin{array}{c}x_{1} \\ y_{1} \\ \theta_{1} \\ \varphi_{1} \\ x_{2} \\ y_{2} \\ \theta_{2} \\ \varphi_{2}\end{array}\right\}$ which maximize JF $(Z) \rightarrow$ Equation 64

Find $Z=\left\{\begin{array}{c}x_{1} \\ y_{1} \\ \theta_{1} \\ \varphi_{1} \\ x_{2} \\ y_{2} \\ \theta_{2} \\ \varphi_{2}\end{array}\right\}$ which maximize $\mathrm{JF}^{\frac{1}{3}}(Z) \rightarrow$ Equation 65 
Constraints for all objective functions, considering the range in Table 2, are:

$$
\begin{aligned}
& \left(\mathrm{x}_{1}-\mathrm{x}_{\mathrm{t} 1}\right)^{2}+\left(\mathrm{y}_{1}-\mathrm{y}_{\mathrm{t} 1}\right)^{2} \geq 40 \\
& \left(\mathrm{x}_{2}-\mathrm{x}_{\mathrm{t} 2}\right)^{2}+\left(\mathrm{y}_{2}-\mathrm{y}_{\mathrm{t} 2}\right)^{2} \geq 40
\end{aligned}
$$

where:

$\mathrm{x}_{\mathrm{t} 1} \rightarrow$ Center point of the tube 1 at axis- $\mathrm{x}$.

$\mathrm{x}_{\mathrm{t} 2} \rightarrow$ Center point of the tube 2 at axis- $\mathrm{x}$.

$y_{t 1} \rightarrow$ Center point of the tube 1 at axis-y.

$y_{t 2} \rightarrow$ Center point of the tube 2 at axis-y.

\subsection{Approaches for optimization}

For optimization procedure adopted herein, two approaches were considered:

1. Direct optimization: CFD model is the "function" and there is no error in the function evaluation by optimizer.

2. Optimization through surrogate model: The function evaluation is done through RSM (Response Surface Methodology), which implies in errors. It is discussed in Appendix B.

A discussion could be raised about the best approach to apply in an optimization problem. Considering numerical analysis, surrogate model is normally used when the time to convergence is much high, for example, either evaluating an automotive crash test or aerodynamic. Direct optimization could be applied in these kinds of problems; however a satisfactory optimal solution could be dependent on the input variable number. For the present work, numerical model is reasonably fast and the direct optimization could be used without major difficulties. Therefore, direct 
optimization seems to be the better choice to find the best solution. However, others features must be analyzed, such as:

a. By surrogate model, it is possible to evaluate the objective function profile for each input variable. This can improve the knowledge about the problem;

b. For each heat exchanger application, there are many performance criterions, Webb (2005). Looking for this, surrogate model could be a better choice because the same RSM could be considered by optimizer for different objective function. However, through direct optimization it would be necessary one optimization process for each objective function. Therefore, surrogate model allows greater flexibility in analysis.

c. Direct optimization is more beneficial when RSM quality is a concern. The higher maximum error between predicted output (from RSM) and real output (from CFD model) more difficult is for the optimizer to find satisfactory optimal objective function. Thus, the RSM quality should be checked before optimization process start, which is presented in Appendix B.

Figure 43 and Figure 44 shows the flowchart used for surrogate model approach and direct optimization approach, respectively. Optimization process using surrogate model approach was done in two steps. First, all models generated by DoE (Design of Experiment) were simulated and the Colburn and Friction factors were calculated. Thereafter, a sheet with input and output data were applied to RSM training and test. Thus, after RSM quality is satisfactory, the optimization process is started. Obviously, the optimal solution found by optimizer is checked by CFD analysis. In this case, the complete analysis is an iterative process. For direct optimization, the optimal solution was found directly in one step. The difficulty considering this approach was due to the calculation of the Colburn and Friction factors from external data-sheet and integrates this to optimizer. Failure connection between local computer and cluster was another issue especially when optimization based on gradient was tested. 


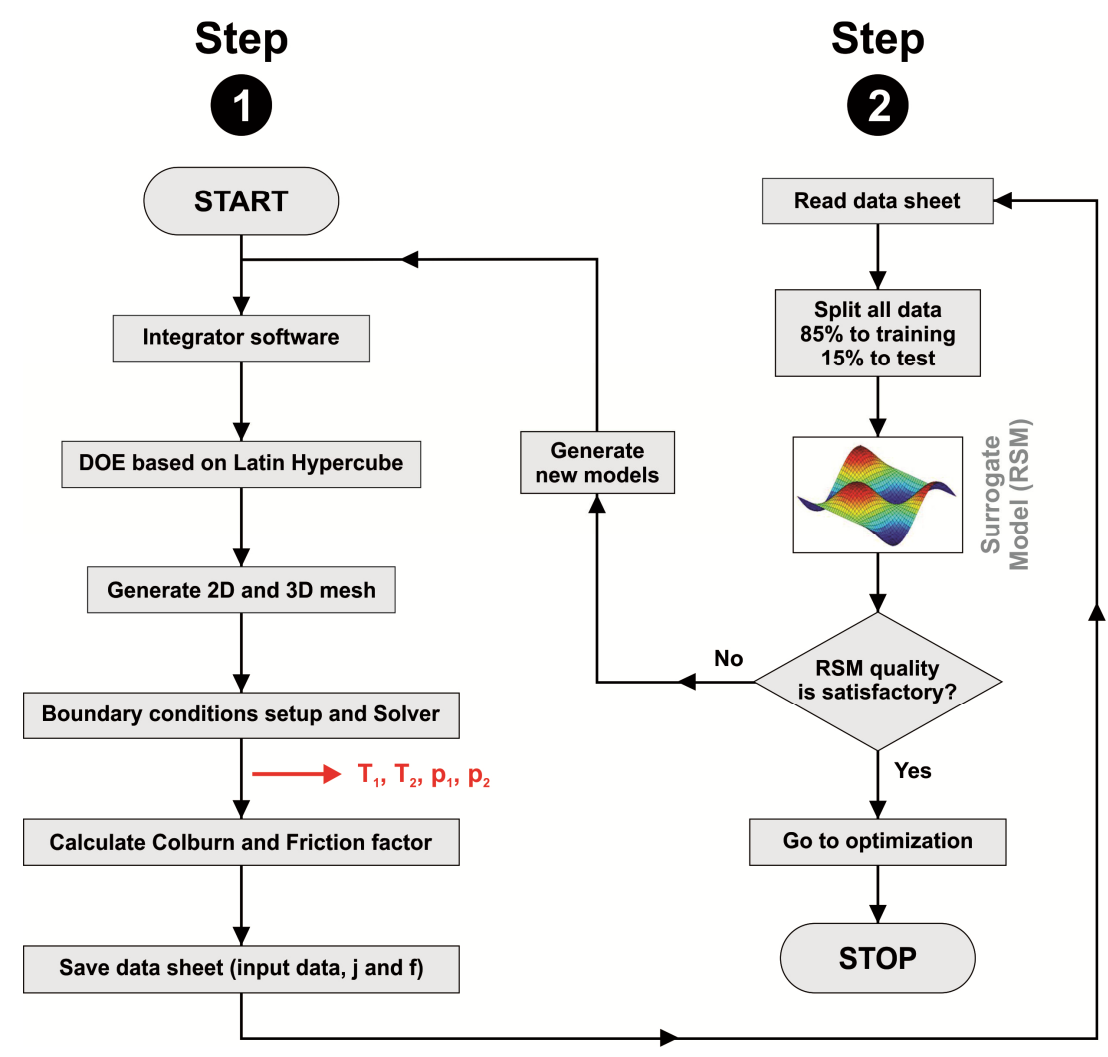

Figure 43 - Flowchart for optimization considering surrogate model in two steps.

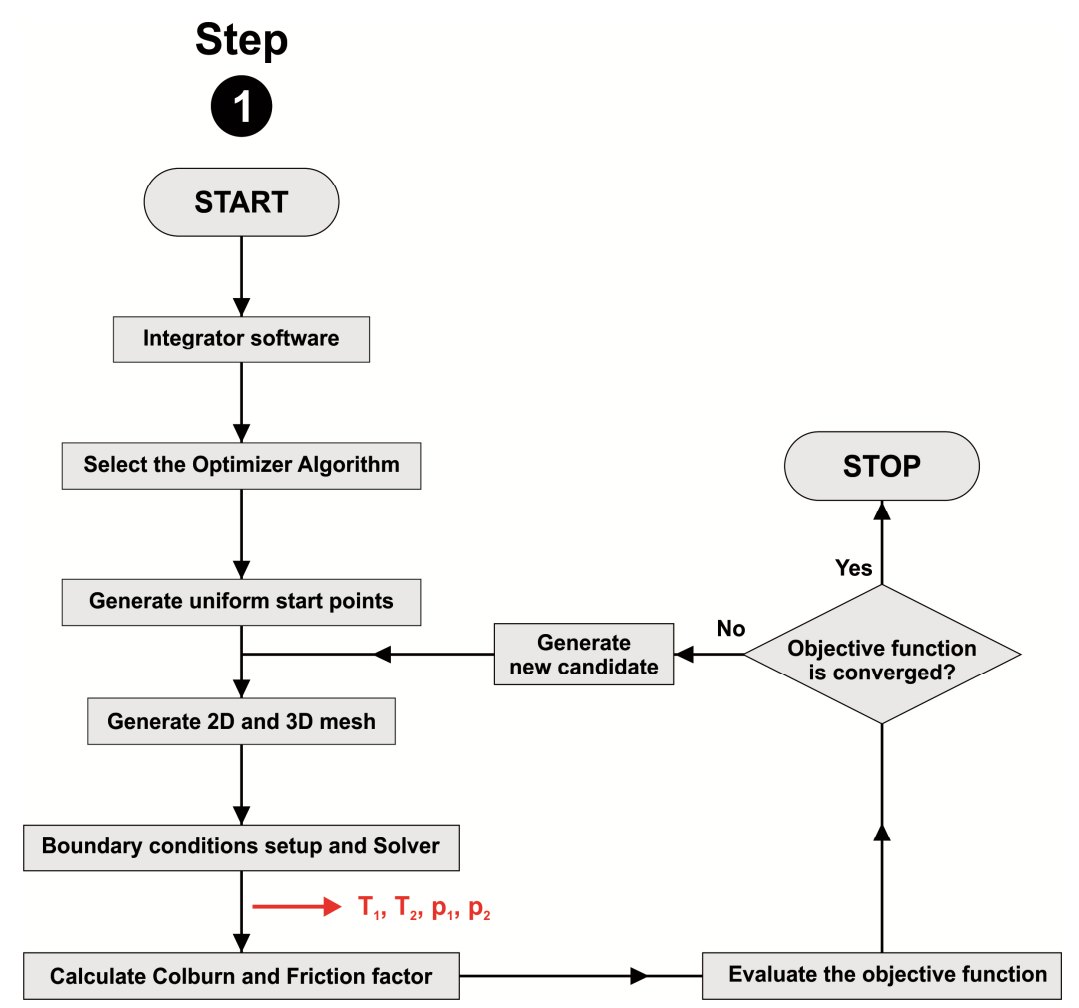

Figure 44 - General flowchart for direct optimization in one step. 
In the next sections are presented the main features of Neural Network (ANN) and Genetic Algorithm (GA), as well as, the practical applications on thermal engineering. ANN and GA are subjects related to the group of Artificial Intelligence (Al). Moreover, the DoE strategy to RSM training is also discussed.

\subsection{Neural Network and Genetic Algorithm}

The last 2 decades have seen enormous strides in technologies that can be loosely grouped together under the general term artificial intelligence (AI). Since, at the same time, relatively powerful desktop computers have become commonly available, applications of these methods are quickly spreading to many areas of science and engineering outside the field of computer science, especially for complex systems where more traditional methods have failed to be useful. The techniques of Artificial Neural Networks (ANNs) and Genetic Algorithms (GAs) have been shown to be particularly useful in the thermal sciences. The engineering discipline of thermal sciences, particularly heat transfer, specifically deals with the analysis, design, and control of such systems, and has a long history of development in response to the needs in a great variety of applications. Much of the advancement in the field was the result of first-principle analyses for the simpler phenomena, supplemented by experimental correlations for the more complex ones for which the first principles are either not known or the problem is too complex for their use. Unfortunately, the existing methods of analyses are no longer adequate to deal with many current applications, even though analyses are still critically needed for a variety of reasons, such as, for instance, energy efficiency, environmental concern, optimum design and control, cost, and competitiveness in the market place. While the thermal sciences as a basic engineering discipline must and should continue to develop and advance our quantitative understanding of the fundamental phenomena involved, new methods of analysis must be found in the interim, which can be used as a surrogate for such understanding, Kreith et al. (2000). 


\subsubsection{Artificial Intelligence (Al)}

Artificial Intelligence (AI) although easy to recognize it is difficult to define. The word artificial is used to mean something man-made, but intelligence itself is a more elusive concept. In fact, one can debate whether machines can actually think. Working definitions would be to say that it is:

"The study of how to make computers do things which, at the moment, people do better", (Rich and Knight, 1991).

"The study that encompasses computational techniques for tasks that apparently requires intelligence when performed by humans", (Tanimoto, 1990).

"The discipline that aims to understand the nature of human intelligence through the construction of computer programs that imitate intelligent behavior", (Bonnet, 1985)

"The study of mental faculties through the use of computational models", (Charniak and McDermott, 1985).

Although every definition has its drawbacks, it appears that some elements of a knowledge base, search techniques, and logical reasoning are usually involved in Al and that the techniques usually attempt a simulation of a specific aspect of human or animal behavior. There are also related and overlapping disciplines that go by other names. Soft computing, for example, is a collection of techniques including fuzzy logic, neural networks, genetic algorithms, and probabilistic reasoning that are based on the idea that "intelligent systems should exploit, whenever possible, the tolerance for imprecision and uncertainty" (Bouchon-Meunier et al., 1995 and Jang et al., 1997). The point is that these techniques are able to deal with complex problems that would be difficult to handle otherwise, Kreith et al. (2000).

Al has a wide variety of applications in the natural and engineering sciences, among which are pattern recognition, decision making, system control, information 
processing, natural languages, symbolic mathematics, computer-aided instruction, speech recognition, vision, and robotics. These applications have spawned a large number of usable algorithms, such as optimization and search techniques, that can be used in other fields. Out of the broad set of techniques available, this thesis was concerned with two specific methods that have been shown to be useful in the thermal sciences: artificial neural networks (ANN) and genetic algorithms (GA).

At the outset one must ask why Al techniques have any role in the thermal sciences. The answer to this lies at the core of the subject, ie, in heat transfer, even considering that the fundamental is well understood; such as the equations for conduction, convection, and radiation, besides the material properties involved can also be measured. A major application of heat transfer is in devices, such as heat exchangers which are one of the basic components of thermal systems. A wide variety of heat exchangers exists in the market and for each is necessary, for design and selection purposes, to know the heat transfer rate under prescribed operating conditions. For a given device exchanging heat between two fluids, the heat transfer rate depends on the flow rates and the inlet temperatures of each fluid. From the heat transfer rate, secondary quantities such as the fluid outlet temperatures can be determined. Through the governing equations, it is extremely difficult to solve them numerically under actual operating condition. Among the reasons for this are: complicated heat and fluid flow geometries, turbulence in the flow, existence of hydrodynamic and thermal entrance regions, non-uniform local heat transfer rates and fluid temperatures, secondary flows in the tube bends, vortices in the neighborhood of the tube-fin junctions, air-side flow development in fin passages, heat conduction along tube walls, natural convection within the tubes and between fins, and temperature dependence of fluid properties, Kreith et al. (2000).

Overall, simply for purposes of discussion, it is defined a complex system to be one that is made up of a large number of simple subsystems that interact with each other. The whole is more than the sum of the parts so that, even given complete understanding of the subsystems and the laws governing their interactions; it is not a trivial matter to calculate the properties or behavior of the whole. On the other hand, if the interactions between the subsystems are weak or nonexistent, the system is decomposable or nearly decomposable, and is much easier to handle. 
Empirical information in the form of correlations is commonly used to help in the analysis of thermal systems that are complex. These correlations usually have little physical basis and are proposed to have the simplest form, that will give the best accuracy. The vagueness in this procedure gives rise to a variety of correlations for even the most straightforward calculation like, for example, that of turbulent heat transfer in a duct. Furthermore, as our ability to carry out complicated calculations for the purpose of design increases, the definition of the "simplest" form changes. At this point in time, it may be said that simplicity in the correlation may no longer be of concern, but accuracy in the predictions is. In this regard, artificial intelligence has much to offer. It is typically used in instances where the ultimate purpose is to detect and make use of patterns, rather than to uncover new physics. Xie et al. (2009) provide an excellent comparison between correlation accuracy and results by ANN. They have shown that ANN is superior regarding accuracy.

\subsubsection{Heat Exchangers}

Reiterating the information outlined, Kreith et al. (2000) showed an interesting example about why apply $\mathrm{Al}$ in thermal systems, and point out some reasons for this.

Empirical information about a given heat exchanger is experimentally determined by the manufacturer and is presented to the user, ie, the thermal system designer, as the heat transfer rate as a function of the four control variables: the two flow rates and the two inlet temperatures. Being a four-variable function, it is difficult to represent completely, although partial information can be provided in the form of charts or graphs. In principle, this function depends on the geometry of the heat exchanger, the materials with which is made, the surface conditions, the fluids used, etc.. It completely characterizes the heat exchanger, and is the information that must be transferred in some form from the manufacturer to the design engineer. Since the raw experimental data would require a large amount of space for data storage, it is necessary to compress the information in some fashion for transfer from manufacturer to user, and from which can later be accurately recovered. There are different ways in which this can be done. In the simplest procedure the information is compressed as two heat transfer correlations, one for the inside and another for the 
outside, thus characterizing the heat exchanger performance by only these two quantities. For each side a characteristic temperature difference is assumed. However, in reality this is found to be inaccurate; the heat transfer coefficients are known to vary considerably with flow rates and fluid properties. Furthermore, without accurate tube wall temperature measurements, which are not easy to make, it is difficult to separate the experimentally determined overall thermal resistance into its internal and external components to find each heat transfer coefficient separately. It is possible to assume separate correlations for the heat transfer on either side and to use a regression analysis to determine the constants. Summing the resistances, however, produces a correlation that is not possible to linearize and, hence, could give more than one set of constants. It can resort to dimensionless analysis to reduce the number of variables involved in the correlation. The internal and external Nusselt (or Stanton) numbers should thus depend on the corresponding Reynolds and Prandtl numbers. A correlation using non-dimensional variables has the advantage that it can be used for a wide range of length scales, flow rates, and fluids. This is the most common procedure.

However, loss of accuracy is the price to be paid for this generality since not all the physical phenomena involved in the heat transfer process, like the previously cited examples show, scale exactly with the limited set of non-dimensional variables used. Property variations are one example; there will be a significant difference in the heat rate predictions if the manufacturer and the user assume different values of the viscosity of the liquid (the viscosity of water, for example, at $40^{\circ} \mathrm{C}$ is $65 \%$ of what it is at $20^{\circ} \mathrm{C}$ ). Letting a characteristic temperature difference scale a temperature profile assumes that the profile will be similar under all conditions, which is not the case. Moreover, the user of the information, the thermal system designer, is usually interested only in the heat transfer rate and not in intermediate variables like the internal and external heat transfer coefficients. It is preferable to sacrifice generality of application and correlate the heat rate directly with respect to the other variables.

The errors that are usually present in the predictions of a heat transfer correlation are due less to errors in measurement than to data compression represented by the correlation. Accuracies of a few percent would be achievable if the predictions had the same error level as the measurements. The two approaches that we will consider in detail to resolve this problem are outlined below. 
1. The artificial neural network technique offers an alternative approach to the problem of information compression. It is a procedure that is usually used to predict the response of a complex physical system that cannot be easily modeled mathematically. The network is first trained by experimentally obtained input-output sets of data, after which can be used for prediction. The manufacturer can train a network using the experimental data; the constants or parameters of the trained network can then be transferred to the user who can calculate the performance of the heat exchanger under any other flow rate or inlet temperature condition.

2. The search for the correlation that best fits the data can be carried out using genetic algorithms. Given a specific form of the correlation, the constants therein can be obtained through the use of a GA. Extending the idea farther, the best correlation function itself can be selected among a restricted class by genetic programming. The objective is not that the form of the correlation be simple, just that its predictions be accurate.

\subsubsection{Artificial Neural Network}

In this section, it is discussed the ANN technique, which is generally considered to be a subclass of $\mathrm{Al}$, and its application to the analysis of complex thermal systems.

The technique is rooted in and inspired by the biological network of neurons in the human brain that learns from external experience, handles imprecise information, stores the essential characteristics of the external input, and generalizes previous experience (Eeckman, 1992). In the biological network of interconnecting neurons, each receives many input signals from other neurons and gives only one output signal which is sent to other neurons as part of their inputs.

The ANN attempts to mimic the biological neural network: the processing unit is the artificial neuron; it has synapses or interneuron connections characterized by synaptic weights; an operator performs a summation of the input signals weighted by the respective synapses; an activation function limits the permissible amplitude range of the output signal. It is also important to realize the essential difference between a 
biologic neural network and ANN. Biological neurons function is much slower than the computer calculations associated with an artificial neuron in an ANN. On the other hand, the delivery of information across the biological neural network is much faster. The biological one compensates for the relatively slow chemical reactions in a neuron by having an enormous number of interconnected neurons doing massively parallel processing, while the number of artificial neurons must necessarily be limited by the available hardware.

Following, in the next section is presented a brief description about the basic principles and characteristics of the ANN, along with the details of the computations made in the feed-forward mode and the associated back-propagation algorithm, which is used for neural networking training. Issues related to the actual implementation of the algorithm were also presented.

\subsubsection{ANN Methodology}

Haykin (1994) present the history of ANN and its mathematical background. Many different definitions of ANNs are possible; the one proposed by Schalkoff (1997) is that an ANN is a network composed of a number of artificial neurons. Each neuron has an input/output characteristic and implements a local computation or function. The output of any neuron is determined by this function, its interconnection with other neurons, and external inputs. The network usually develops an overall functionality through one or more forms of training; this is the learning process. Many different network structures and configurations have been proposed along with their own methodologies of training (Warwick et al., 1992).

\subsubsection{Feed-forward Network}

There are many different types of ANNs, but one of the most appropriate for engineering applications is the supervised, fully connected multilayer configuration 
(Zeng, 1998), in which learning is accomplished by comparing the output of the network with the data used for training.

Figure 45 shows an ANN consisting of a series of layers, each with a number of nodes. The first and last layers are for input and output, respectively, while the others are the hidden layers. The network is said to be fully connected when any node in a given layer is connected to all the nodes in the adjacent layers, Kreith et al. (2000).

node number

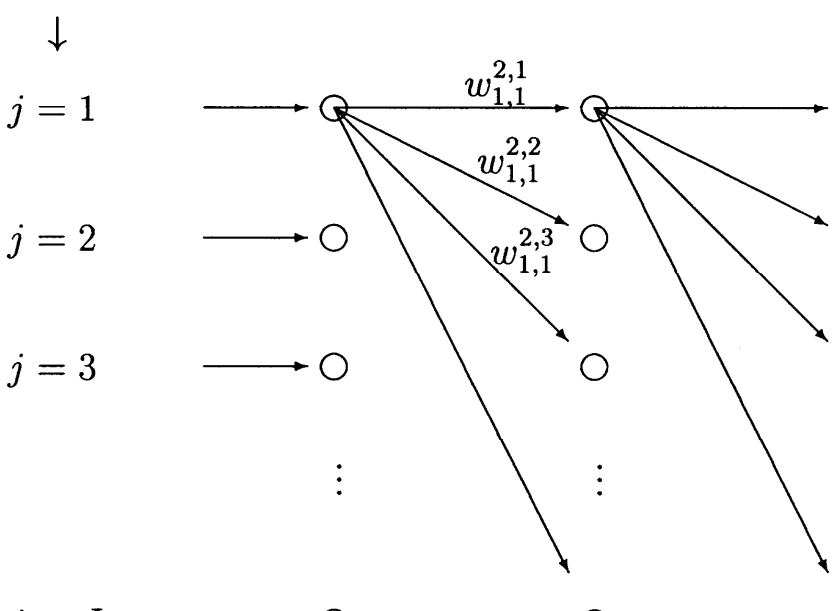

$j=J_{i} \longrightarrow \mathrm{O}$

layer number $\rightarrow \quad i=1$

$$
i=2
$$

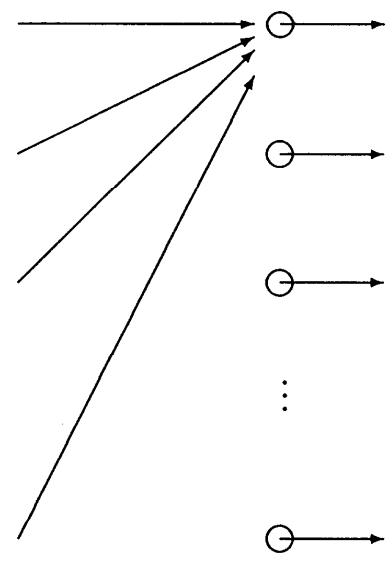

$i=I$

Figure 45 - Schematic of a fully connected multilayer ANN, Kreith et al. (2000).

About the Figure $45,(i, j)$ is the $j_{\text {th }}$ node in the $i_{\text {th }}$ layer. The line connecting a node $(\mathrm{i}, \mathrm{j})$ to another node in the next layer $\mathrm{i}+1$ represents the synapse between the two nodes. $x_{i, j}$ is the input of the node $(i, j), y_{i, j}$ is its output, $\theta_{i, j}$ is its bias, and $w_{i-1, k}^{i, j}$ is the synaptic weight between nodes $(i-1, k)$ and $(i, j)$. The total number of layers, including those for input and output is $(\mathrm{I})$, and the number of nodes in the $\mathrm{i}_{\mathrm{th}}$ layer is $J_{i}$. The input information is propagated forward through the network; $J_{1}$ values enter in the network and $J_{1}$ leave. The flow of information through the layers is a function of the computational processing occurring at every internal node in the network. The 
relation between the output of node $(i-1, k)$ in one layer and the input of node $(i, j)$ in the following layer is defined by Equation 70 :

$$
x_{i, j}=\theta_{i, j}+\sum_{k=1}^{J_{i-1}} w_{i-1, k}^{i, j} y_{i-1, k}
$$

Thus, the input $x_{i, j}$ of node $(i, j)$ consists of a sum of all the outputs from the previous nodes modified by the respective internodes synaptic weights $w_{i-1, k}^{i, j}$ and a bias $\theta_{i, j}$. The weights are characteristics of the connection between the nodes and the bias of the node itself. The bias represents the propensity for the combined incoming input to trigger a response from the node and presents a degree of freedom which gives additional flexibility in the training process. Similarly, the synaptic weights are the weighting functions which determine the relative importance of the signals originated from the previous nodes.

The input and output of the node $(\mathrm{i}, \mathrm{j})$ are related by Equation 71 :

$$
\mathrm{y}_{\mathrm{i}, \mathrm{j}}=\phi_{\mathrm{i}, \mathrm{j}}\left(\mathrm{x}_{\mathrm{i}, \mathrm{j}}\right)
$$

where $\phi_{i, j}(x)$ is called the activation or threshold function, which plays the role of the biological neuron determining whether it should fire or not on the basis of the input to that neuron. A schematic of the nodal operation is shown in Figure 46. It is obvious that the activation function plays a central role in the processing of information through the ANN. Keeping in mind the analogy with the biological neuron, when the input signal is small the neuron suppresses the signal altogether, resulting in a vanishing output, and when the input exceeds a certain threshold the neuron fires and sends a signal to all the neurons in the next layer. This behavior is determined by the activation function. Several appropriate activation functions have been studied (Haykin, 1994; Schalkoff, 1997). For instance, a simple step function can be used, but the presence of non-continuous derivatives causes computing difficulties. The 
most popular one is the logistic sigmoid function, Equation 72, which is applied in ModeFrontier software.

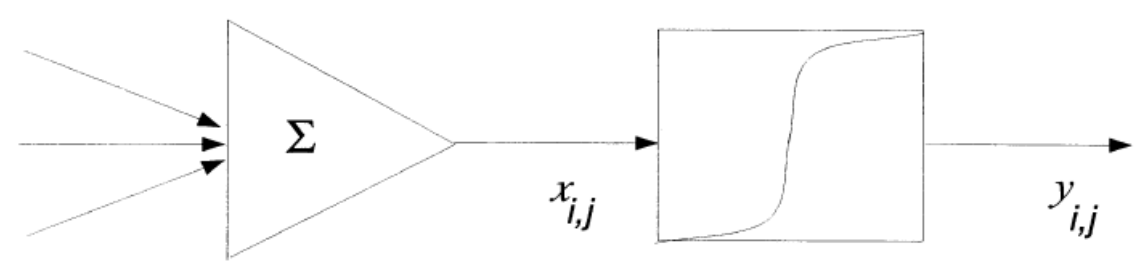

Figure 46 - Nodal operation in an ANN, Kreith et al. (2000).

$$
\phi_{i, j}(\xi)=\frac{1}{1+e^{-\frac{\xi}{c}}}
$$

where $\xi$ is the variable in sigmoid function.

For $\mathrm{i}>1$, where $\mathrm{c}$ determines the steepness of the function.

For $\mathrm{i}=1, \phi_{i, j}(\xi)=\xi$ is used instead.

The sigmoid function is an approximation to the step function, but with continuous derivatives. The nonlinear nature of the sigmoid function is particularly beneficial in the simulation of practical problems. For any input $x_{i, j}$, the output of a node $y_{i, j}$ always lies between 0 and 1 . Thus, from a computational point of view, it is desirable to normalize all the input and output data with the largest and smallest values of each of the data sets; however, this does not guarantee a better surface response. Xie et al. (2009) proposed a scale for input/output variables through the transformations, Equation 73:

$$
\phi_{i}^{\mathrm{NORM}}=\frac{\phi_{\mathrm{i}}-\phi_{\mathrm{i}, \text { min }}}{\phi_{\mathrm{i}, \text { max }}-\phi_{\mathrm{i}, \text { min }}}\left(\mathrm{S}_{\max }-\mathrm{S}_{\min }\right)+\mathrm{S}_{\text {max }}
$$


where $S_{\max }$ and $S_{\min }$ are 1.0 and 0.0 , respectively, $\phi_{i, \max }$ and $\phi_{i, \text { min }}$ are the maximum and minimum values of individual input/output variable. Xie et al. (2009) pointed out that the computational difficulty could be verified considering these $S_{\max }$ and $S_{\min }$ values and proposed, based on previous experience, the values of 0.85 and 0.15 , respectively. However, ModeFrontier software consider 1 and 0 for $S_{\max }$ and $S_{\min }$, respectively.

\subsubsection{ANN training}

For a given network, the weights and biases must be adjusted for known input-output values through a process known as training. The back-propagation method is a widely used deterministic training algorithm for this type of ANN (Rumelhart et al., 1986). The central idea of this method is to minimize an error function by the method of steepest descent to add small changes in the direction of minimization. This algorithm may be found in many texts on ANN (for instance, Rzempoluck, 1998), and only a brief outline will be given here based on Kreith et al. (2000).

In usual complex thermal-system applications where no physical models are available, appropriate training data come from either experiments or numerical simulation. The first step in the training algorithm is to assign initial values to the synaptic weights and biases in the network based on the chosen ANN configuration. The second step is to initiate the feed-forward of information starting from the input layer. In this manner, successive input and output of each node in each layer can all be computed. When finally $i=1$, the value of $y_{1, j}$ will be the output of the network. Training of the network consists of modifying the synaptic weights and biases until the output values differ little from the experimental data which are the targets. This is done by means of the back-propagation method. First an error $\delta_{1, j}$ is quantified by Equation 74:

$$
\delta_{1, j}=\left(t_{1, j}-y_{1, j}\right) y_{1, j}\left(1-y_{1, j}\right)
$$


where $t_{1, j}$ is the target output for the $j$-node of the last layer. The above equation is simply a finite difference approximation of the derivative of the sigmoid function. After calculating all $\delta_{1, j}$, the computation then moves back to the layer $1-1$. Since the target outputs for this layer not exist, a surrogate error is used instead for this layer defined as Equation 75:

$$
\delta_{I-1, k}=y_{I-1, k}\left(1-y_{I-1, k}\right) \sum_{j=1}^{J_{I}} \delta_{l, j} w_{l-1, k}^{l, j}
$$

A similar error $\delta_{i, j}$ is used for all the rest of the inner layers. These calculations are then continued layer by layer backward until layer 2. It is seen that the nodes of layer 1 have neither $\delta$ nor $\theta$ values assigned, since the input values are all known and invariant. After all the errors $\delta_{1, j}$ are known, the changes in the synaptic weights and biases can then be calculated by the generalized delta rule (Rumelhart et al., 1986), Equations 76 and 77 :

$$
\begin{aligned}
& \Delta \mathrm{w}_{\mathrm{i}-1, \mathrm{k}}^{\mathrm{i} j}=\lambda \delta_{\mathrm{i}, \mathrm{j}} \mathrm{y}_{\mathrm{i}-1, \mathrm{k}} \\
& \Delta \theta_{\mathrm{i}, \mathrm{j}}=\lambda \delta_{\mathrm{i}, \mathrm{j}}
\end{aligned}
$$

For $\mathrm{i}<1$, from which all the new weights and biases can be determined. The quantity $\lambda$ is known as the learning rate that is used to scale down the degree of change made to the nodes and connections. The larger the training rate, the faster the network will learn, but the chances of the ANN to reach the desired outcome may become smaller as a result of possible oscillating error behaviors. Small training rates would normally imply the need for longer training to achieve the same accuracy.

A cycle of training consists of computing a new set of synaptic weights and biases successively for all the experimental runs in the training data. The calculations are then repeated over many cycles while recording an error quantity $E$ for a given run within each cycle, Equation 78: 


$$
E=\frac{1}{2} \sum_{j=1}^{J_{1}}\left(t_{l, j}-y_{l, j}\right)^{2}
$$

The output error of the ANN at the end of each cycle can be based on either a maximum or averaged value for a given cycle. Note that the weights and biases are continuously updated throughout the training runs and cycles. The training is terminated when the error of the last cycle, barring the existence of local minima, falls below a prescribed threshold. The final set of weights and biases can then be used for prediction purposes, and the corresponding ANN becomes a model of the inputoutput relation of the thermal-system problem.

\subsubsection{Implementation Issues}

In this thesis, the ANN implementation was not a concern, because the choice herein was to use of commercial software (ModeFrontier) and, thus, drive the attention on augmentation heat transfer phenomenology. However Kreith et al. (2000) point out some implementation issues, regarding several uncertain choices, which include the number of hidden layers, the number of nodes in each layer, the initial assignment of weights and biases, the training rate, the minimum number of training data sets and runs, the learning rate, and the range within which the input-output data are normalized. Such choices are by no means trivial, and are rather important in achieving good ANN results. Since there is no general sound theoretical basis for specific choices, past experience and numerical experimentation are still the best guides, despite the fact that much research is now going on to provide a rational basis (Zeng, 1998).

On the issue of number of hidden layers, there is a sufficient, but certainly not necessary, theoretical basis known as the Kolmogorov's mapping neural network existence theorem as presented by Hecht-Nielsen (1987), which essentially stipulates that only one hidden layer of artificial neurons is sufficient to model the input-output relations as long as the hidden layer has $2 J_{1}+1$ nodes. ModeFrontier software has been considered this approach in its implementation and there is not 
another ANN structures enable in. The number of hidden layers and neurons can vary depending on the size and nature of the data set. Since in realistic problems involving a large set of input parameters the nodes in the hidden layer would be excessive to satisfy this requirement, the general practice is to use two hidden layers as a starting point, and then to add more layers as the need arises, while keeping a reasonable number of nodes in each layer (Flood and Kartam, 1994).

A slightly better situation is in the choice of the number of nodes in each layer and in the entire network. Increasing the number of internal nodes provides a greater capacity to fit the training data. In practice, however, too many nodes suffer the same fate as the polynomial curve-fitting routine by collocation at specific data points in which the interpolations between data points may lead to large errors. In addition, a large number of internal nodes slow down the ANN both in training and in prediction. One suggestion given by Rogers (1994) and Jenkins (1995) is that, Equation 79:

$$
N_{t}=1+N_{n} \frac{J_{1}+J_{1}+1}{J_{l}}
$$

where $N_{t}$ is the number of training data sets, and $N_{n}$ is the total number of internal nodes in the network. If $\mathrm{N}_{\mathrm{t}}, \mathrm{J}_{1}$ and $\mathrm{J}_{1}$ are known in a given problem, the above equation determines the suggested minimum number of internal nodes. Also, if $N_{n}$, $J_{1}$ and $J_{1}$ are known, it gives the minimum value of $N_{t}$. The number of data sets used should be larger than that given by this equation to ensure the adequate determination of the weights and biases in the training process. Other suggested procedures for choosing the parameters of the network include the one proposed by Karmin (1990) by first training a relatively large network that is then reduced in size by removing nodes which do not significantly affect the results, and the so-called Radial-Gaussian system which adds hidden neurons to the network in an automatic sequential and systematic way during the training process (Gagarin et al., 1994). Also available is the use of evolutionary programming approaches to optimize ANN configurations (Angeline et al., 1994). Some authors (see, for example, Thibault and Grandjean, 1991) present studies of the effect of varying these parameters. 
Moreover, by Equation 80, Hush (1989), from a series of experiments, suggest an expression for a minimum training data set to avoid underfitting.

$$
\mathrm{N}_{\text {training }}=30 \mathrm{~N}_{\mathrm{i}}\left(\mathrm{N}_{\mathrm{i}}+1\right)
$$

Equation 80 was considered as a start assessment for DOE and, considering the input data mentioned in Chapter 1, the initial training data set must be at least 2160 models. However, overfitting should be avoided, as broadly discussed by Baum (1989) and Krose and Smagt (1996).

The issue of assigning the initial synaptic weights and biases is less certain. Despite the fact that better initial guesses would require less training efforts, or even less training data, such initial guesses are generally unavailable in applying the ANN analysis to a new problem. The initial assignment then normally comes from a random number generator of bounded numbers. Unfortunately, this DoEs does not guarantee that the training will converge to the final weights and biases for which the error is a global minimum. Also, the ANN may take a large number of training cycles to reach the desired level of error. Wessels and Barnard (1992), Drago and Ridella (1992), and Lehtokangas et al. (1995) suggested other methods for determining the initial assignment so that the network converges faster and avoids local minima. On the other hand, when the ANN needs upgrading by additional or new experimental data sets, the initial weights and biases are simply the existing ones.

During the training process, the weights and biases continuously change as training proceeds in accordance with Equations 76 and 77, which are the simplest correction form to use. Other possibilities, however, are also available (Kamarthi et al., 1992). The choice of the training rate $\lambda$ is largely by trials. It should be selected to be as large as possible, but not too large to lead to non-convergent oscillatory error behaviors. Finally, since the sigmoid function has the asymptotic limits of $[0,1]$ and may thus cause computational problems in these limits, is desirable to normalize all physical variables into a more restricted range such as $[0.15,0.85]$. The choice is somewhat arbitrary. However, pushing the limits closer to $[0,1]$ may commonly produce more accurate training results at the expense of larger computational efforts. As already mentioned, the normalization could be done by Equation 73 . 


\subsubsection{Additional Applications in Thermal Engineering}

In the past applications of ANN to engineering problems have been attempted in structural engineering and engineering mechanics (Zeng, 1998). Some studies of applying ANN to problems in thermal systems have been carried out as following, according to Kreith et al. (2000). With the exception of neural network system and control systems applied to HVAC systems, the studies have been somewhat sporadic and in only some distinct areas of application. For heat transfer data analysis and correlation, an ANN based methodology was proposed by Thibault and Grandjean (1991) and a similar methodology was introduced by Jambunathan et al. (1996) to predict coefficients of heat transfer in convective flow systems using liquid crystal thermography. Both steady and unsteady heat conduction problems were treated by ANN in studies by Gobovic and Zaghloul (1993), Yentis and Zaghloul (1994), and Kuroe and Kimura (1995). Kaminski et al. (1996) gave an interesting description of the thermal deterioration process based on combined ANN and GA analysis. An ANN was also used to identify location and strength of unknown heat sources by sparse temperature measurements (Momose et al., 1993). In addition, an ANN-based attempt was made to predict measured intrinsic thermodynamic properties (Normandin et al., 1993). ANN analyses have also been utilized to study and predict the performance of specific thermal devices and systems. Good estimates of the thermal storage loads and the dynamic system operation of typical thermal storage systems were obtained by Ferrano and Wong (1990) and Ito et al. (1995). Heat exchanger performance and control were studied by Diaz et al. (1996, 1998, 1999), Lavric et al. (1993, 1994), and Bittanti and Piroddi (1997). Several industrial applications of ANN were also demonstrated, in a fluidized-bed dryer (Zbicinski et al., 1996), in a liquid-sodium reflux-pool boiler solar receiver (Fowler et al., 1997), in a steel annealing furnace (Pican et al., 1998), and in the design of a chemical injection-system retrofit fuzzy control system in a thermal power plant (Moon and Cho, 1996). Other specific applications include manufacturing and materials processing involving microelectronic manufacturing (Mahajan and Wang, 1993), a coordinate grinder (Yang et al., 1995), rapid thermal processing control (Fortuna et al., 1996), sensors and sensor analysis involving thermal image processing (Naka et al., 1993), and blast furnace probe temperatures (Bulsari and 
Saxin, 1995). Dhar et al. (1995) used an ANN with wavelet basis functions to predict hourly heating and cooling energy use in commercial buildings with the temperature as the only weather variable. Inoue et al. (1995) studied thermal comfort and climate control. Kawashima et al. (1995) did a 24h thermal load prediction. Li et al. (1996) developed a fault diagnosis method for heating systems using neural networks. Matsumoto et al. $(1996,1997)$ studied the effect of pin fin arrangement on endwall heat transfer. Takemori et al. (1991) worked on a neural network air-conditioning system for individual comfort. Dounis et al. (1992) implemented an Al technique in thermal comfort control for passive solar buildings.

All the studies above mentioned represent some successful attempts to apply the ANN analysis in thermal system problems. Since the good results were obtained so far, there is no reason to expect that the ANN approach cannot be applied to many other thermal problems with equal success, particularly in enhancement heat transfer in compact heat exchanger by longitudinal vortex generator.

\subsubsection{Genetic Algorithms}

Evolutionary programming, of which genetic algorithms (GA) and programming are examples, allow programs to change or evolve as they compute. GAs, specifically, are based on the principle of Darwinian selection. One of their most important applications in the thermal sciences is in the area of optimization of various kinds.

Optimization by itself is fundamental to many applications. In engineering, for example, it is important to the design of systems; analysis permits the prediction of the behavior of a given system, but optimization is the technique that searches among all possible designs of the system to find the one that is the best for the application. The importance of this problem has given rise to a wide variety of techniques which help search for the optimum. There are searches that are gradientbased and those that are not, Russell and Norvig (1995).

In the former the search for the optimum solution, as for example the maximum of a function of many variables, starts from some point and directs itself in an incremental fashion towards the optimum; at each stage the gradient of the function surface 
determines the direction of the search. Local optima can be found in this way, the search for global optimum being more difficult. Again, if one visualizes a multivariable function, it can have many peaks, any one of which can be approached by a hillclimbing algorithm. To find the highest of these peaks, the entire domain has to be searched; the narrower this peak the finer the searching "comb" must be. For many applications this brute force approach is too expensive in terms of computational time. Alternatives, like simulated annealing, are techniques that have been proposed, and the GA is one of them, Kreith et al. (2000).

\subsubsection{GA Methodology}

GAs are discussed in detail by Holland (1975, 1992), Mitchell (1997), Goldberg (1989), Michalewicz (1992), and Chipperfield (1997). One of the principal advantages of this method is its ability to pick out a global extreme in a problem with multiple local extreme. For example, finding the maximum of a function $f(x)$ in a given domain $\mathrm{a} \leq \mathrm{x} \leq \mathrm{b}$. In outline, the steps of the procedure are the following:

- First, an initial population of $n$ members, $x_{1}, x_{2}, \ldots, x_{n} \in[a, b]$ is randomly generated.

- Then, for each $x$ fitness is evaluated. The fitness of effectiveness is the parameter that determines how good the current $x$ is in terms of being close to an optimum. Clearly, in this case the fitness is the function $f(x)$ itself, since the higher the value of $f(x)$ the closer are to the maximum.

- The probability distribution for the next generation is found based on the fitness values of each member of the population. Pairs of parents are then selected on the basis of this distribution.

- The offspring of these parents are found by reproduction, crossover and mutation. These operators create a new generation with a population that is more likely to be fitter than the previous generation. 
- The process is continued as long as desired or until the largest fitness in a generation DoEs does not change much anymore.

The procedure can be generalized for a function of many variables.

Reproduction is an operator where an old design is copied into the new population according to the design's fitness. There are many different strategies to implement this reproduction operator. This is also called the selection process. This operator is not enabling in ModeFrontier software.

Crossover corresponds to allowing selected members of the new population to exchange characteristics of their designs among themselves. Crossover entails selection of starting and ending positions on a pair of randomly selected data, and simply exchanging the data of 0 and 1 between these positions.

Mutation is the third step that safeguards the process from a complete premature loss of valuable genetic material during reproduction and crossover. In terms of a binary string, this step corresponds to selection of a few members of the population, determining a location on the strings at random, and switching the 0 to 1 or vice versa.

Crossover and Mutation were important rules during optimization process, especially considering the approach by surrogate model, obviously because the RSM errors. In such cases, the VG arrangement from optimizer was converged and so checked in CFD model. Thus, these operators were taken carefully.

The foregoing three steps are repeated for successive generations of the population until no further improvement in fitness is attainable. The member in this generation with the highest level of fitness is taken as the optimum design. 


\subsubsection{Additional Applications in Thermal Engineering}

Although the GA is a relatively new technique in relation to its application to thermal engineering, there are a number of different applications that have already been successful, according to Kreith et al. (2000). Davalos and Rubinsky (1996) adopted an evolutionary-genetic approach for numerical heat-transfer computations. Shape optimization is another area that has been developed. Fabbri (1997) used a GA to determine the optimum shape of a fin. The two-dimensional temperature distribution for a given fin shape was found using a finite-element method. The fin shape was proposed as a polynomial, the coefficients of which have to be calculated. The fin was optimized for polynomials of degree 1 through 5 . Von Wolfersdorf et al. (1997) did shape optimization of cooling channels using GAs. The design procedure is inherently an optimization process. Androulakis and Venkatasubramanian (1991) developed a methodology for design and optimization that was applied to heat exchanger networks; the proposed algorithm was able to local solutions where gradient-based methods failed. Abdel-Magid and Dawoud (1995) optimized the parameters of an integral and a proportional-plus-integral controller of a reheat thermal system with GAs. The fact that the GAs can be used to optimize in the presence of variables that take on discrete values was put to advantage by Schmit et al. (1996) who used it for the design of a compact high-intensity cooler.

The placing of electronic components as heat sources is a problem that has become very important from the point of view of computers. Queipo et al. (1994) applied GAs to the optimized cooling of electronic components. Tang and Carothers (1996) showed that the GA worked better than some other methods for the optimum placement of chips. Queipo and Gil (1997) worked on the multi-objective optimization of component placement and presented a solution methodology for the collocation of convectively and conductively air-cooled electronic components on planar printed wiring boards. Meysenc et al. (1997) studied the optimization of microchannels for the cooling of high-power transistors.

Inverse problems may also involve the optimization of the solution. Allred and Kelly (1992) modified the GA for extracting thermal profiles from infrared image data which can be useful for the detection of malfunctioning electronic components. Jones et al. 
(1995) used thermal tomographic methods for the detection of inhomogeneities in materials by finding local variations in the thermal conductivity. Raudensky et al. (1995) used the GA in the solution of inverse heat conduction problems. Okamoto et al. (1996) reconstructed a three-dimensional density distribution from limited projection images with the GA. Wood (1996) studied an inverse thermal field problem based on noisy measurements and compared a GA and the sequential function specification method. Li and Yang (1997) used a GA for inverse radiation problems. Castrogiovanni and Sforza $(1996,1997)$ studied high-heat flux-flow boiling systems using a numerical method in which the boiling-induced turbulent eddy diffusivity term was used with an adaptive GA closure scheme to predict the partial nucleate boiling regime. Hilbert et al. (2006) performed a multi-objective design optimization concerning the blade shape of a heat exchanger, considering the coupled solution of the flow/heat transfer processes applied GA. The aim of the procedure is to find the geometry most favorable to simultaneously maximize heat exchange while obtaining a minimum pressure loss.

\subsubsection{General Discussion}

The evolutionary programming method has the advantage that a functional form of the relationship is obtained. As it can be seen, GA is a successfully techniques from the perspective of thermal engineering, and we can only expect the applications to grow. There are a number of areas in prediction, control, and design where these techniques can be effectively used. One of these, in which progress can be expected, is in thermal networks, Kreith et al. (2000). Thermal networks are complex systems built up from a large number of simple components; although the behavior of each component may be well understood, the behavior of the network requires massive computations that may not be practical. Optimization of networks is an important issue from the perspective of design, since it is not obvious what the most energy-efficient network, given certain constraints, should be. The constraints are usually in the form of the locations that must be served and the range of thermal loads that are needed at each position. A search methodology based on the calculation of every possible network configuration would be very expensive in terms 
of computational time. An alternative based on evolutionary techniques would be much more practical. Under this procedure a set of networks that satisfy the constraints would be proposed as candidates for the optimum. From this set a new and fit generation would evolve and the process repeated until the design not change a lot. The definition of fitness, for this purpose, would be based on the energy requirements of the network.

Calculating the possibilities of the solutions presented in this work, eight independent inputs for the range shown in Table 2 were considered. For position the step adopted was $0.1 \mathrm{~mm}$ and for angles was $1^{\circ}$. Thus, the order of the all possibilities could be around $1.05^{+17}$. These analyses of possibilities are impossible for both numerical and experimental techniques. Therefore, the both approaches adopted herein are very interesting and necessary to apply in optimization problems.

Overall, artificial intelligence techniques are well suited to complex problems, and thermal engineering presents many examples of this. It is not a substitute, but rather a complement, for incisive physical insight and first-principle analyses.

\subsection{Design of Experiment (DoE) and RSM training}

Design of Experiments (DoE) is a methodology that maximizes the knowledge gained from experimental data. Originated in 1920 by a British scientist, Sir R. A. Fisher, it provides a strong tool to design and analyze experiments; eliminating redundant observations and reduces the time and resources to making the experiments. Hence, DoE techniques allow to researcher try to extract, whenever possible, information from a limited number of test runs.

Design of Experiments methodologies are used for different applications:

1. To create samplings for sensitivity analysis, the use of DoE is important in experimental to identify the input variables most affect the response.

2. To create a set of stochastic points for robustness and reliability analysis.

3. To generate an appropriate set of support points for response surface. 
4. To provide an optimization algorithms as initial population of designs.

In this thesis, Latin Hypercube Sampling (LHS) Methodology was applied. LHS was first proposed by McKay et al. (1979). By LHS, for each variable, the points were randomly and uniformly distributed. This methodology is useful when a random sample is needed. It guarantees to be relatively uniformly distributed over each dimension. Thus, by LHS a data set was generated for RSM training and quality test.

As it already mentioned, the training data is the largest set and is used by the neural network to learn the patterns present in the data. Kaastra and Boyd (1996) suggest that the test data ranging in size from $10 \%$ to $30 \%$ of the data set. Therefore, in this present work it was adopted $85 \%$ of data set for RSM training and $15 \%$ for RSM testing, which was randomly selected. The best RSM was select for perform the best test data set. On time, the RSM quality was checked through three indicators: Regression $\left(\mathrm{R}^{2}\right)$, Mean Relative Error $\left(\mathrm{e}_{\text {mean }}\right)$ and Maximum Relative Error $\left(\mathrm{e}_{\max }\right)$. Relative errors are given by the ratio between the absolute residual and the real value. The regression is calculated by Equation 81.

$$
R^{2}=1-\frac{\sum_{i=1}^{n}\left(\phi_{i}-\tilde{\phi}_{i}\right)^{2}}{\sum_{i=1}^{n}\left(\phi_{i}-\bar{\phi}_{i}\right)^{2}}
$$

where:

$\phi_{\mathrm{i}} \rightarrow$ Real value.

$\tilde{\phi}_{\mathrm{i}} \rightarrow$ Predicted value by RSM.

$\bar{\phi}_{\mathrm{i}} \rightarrow$ Mean of all real values.

Herein, is point out an important issue raised by Papila and Raftka (2000), indicating that a substantial amount of noise and existence of outliers could substantially degrade the accuracy of the RSM. However, considering a numerical analysis, this is 
not ease to identify the data that are either outliers or non-linearity, which is intrinsic for each problem. Outlier is a common term in physical experiments, but is not directly applied for computational experiments.

Results of physical experiments are almost always subjected to experimental error. These departures from the 'true' result from three main sources:

- Human error, that is simply error introduced by the experimenter when making a mistake;

- Systematic error, due to a flaw in the philosophy of the experiment that adds a consistent bias to the result;

- Random error, which is due to measurement inaccuracies inherent to the instruments being used.

Computational experiments are also subject to experimental error, resulting from:

- Human error, 'bugs' in the analysis code, incorrectly entered boundary conditions in the solution of a partial differential equation, etc., and;

- Systematic error. For example, an inviscid mathematical model of the viscous flow around a body (an approximation sometimes made for computing time saving purposes) will consistently underestimate the drag forces acting on the body. Another example is the error caused by the inherently finite resolution of the numerical modeling process (eg errors caused by insufficient mesh resolution in a finite element solve). While this type of error can lead to underestimates or overestimates, it will do so in exactly the same way if it repeats the experiment.

Therefore, the difference compared to physical experiments is that computational experiments are not affected by random error - it is deterministic. Since the human error and systematic error was checked (Chapter 3 ), there is a great confidence level in computational results. Therefore, two approaches were tested considering the 
training data; the first approach the "outliers" were removed for RSM training and the second approach the "outliers" were kept in.

An "outlier" is a point that DoEs does not appear to fall within the expected distribution for a particular set and this DoEs does not follow the characteristics of the response data, which is defined in Equation 82.

$$
\begin{aligned}
& \phi<Q_{1}-1.5 \star I Q R \\
& \phi>Q_{3}+1.5 * I Q R
\end{aligned}
$$

where:

$\phi \rightarrow$ Could be Friction factor $(\mathrm{f})$ and Colburn factor $(\mathrm{j})$.

$Q_{1} \rightarrow$ First or lower quartile along with the median (25th percentile).

$\mathrm{Q}_{3} \rightarrow$ Third or upper quartile along with the median (75th percentile).

$\mathrm{IQR} \rightarrow$ Interquartille range: $\mathrm{Q}_{3}-\mathrm{Q}_{1}$

Figures 47 and 48 shows the probability density of the training data set $(85 \%$ of the total data) of Fraction factor (f) and Colburn factor (j) at $\operatorname{Re}=250$ and $\operatorname{Re}=1400$, respectively. 


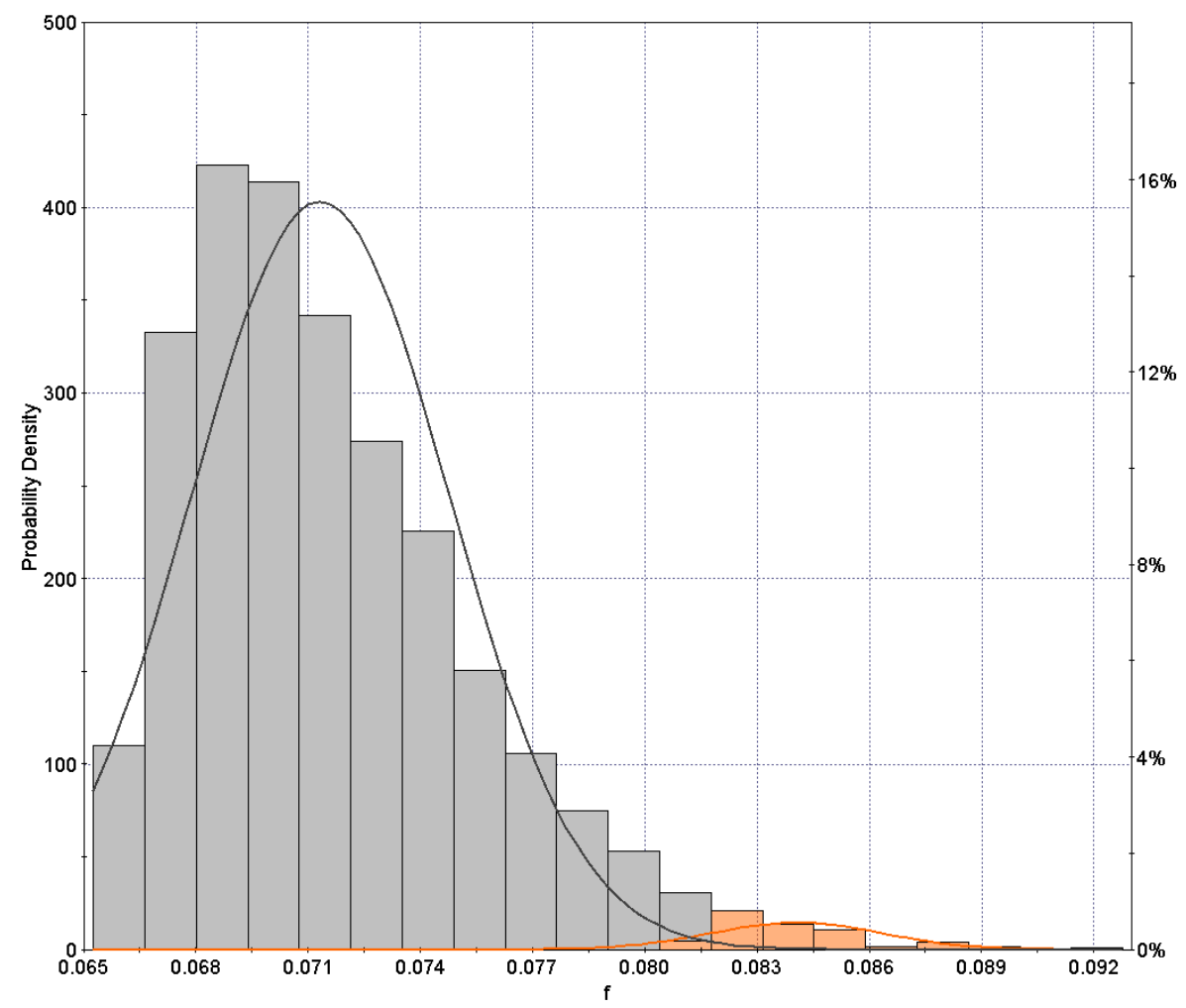

(a)

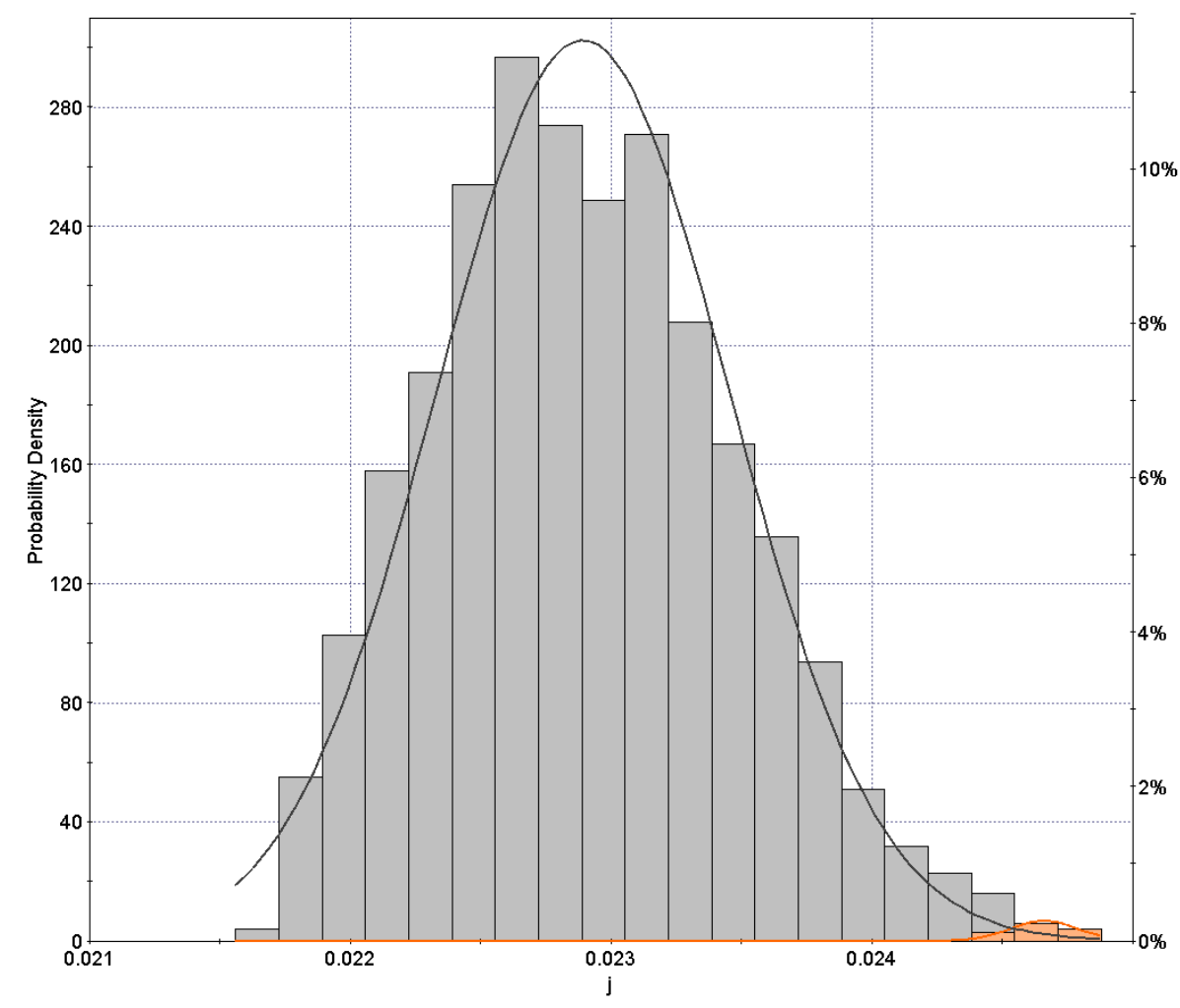

(b)

Figure 47 - Probability density at $\operatorname{Re}=250$ (a) Friction factor and, (b) Colburn factor. 


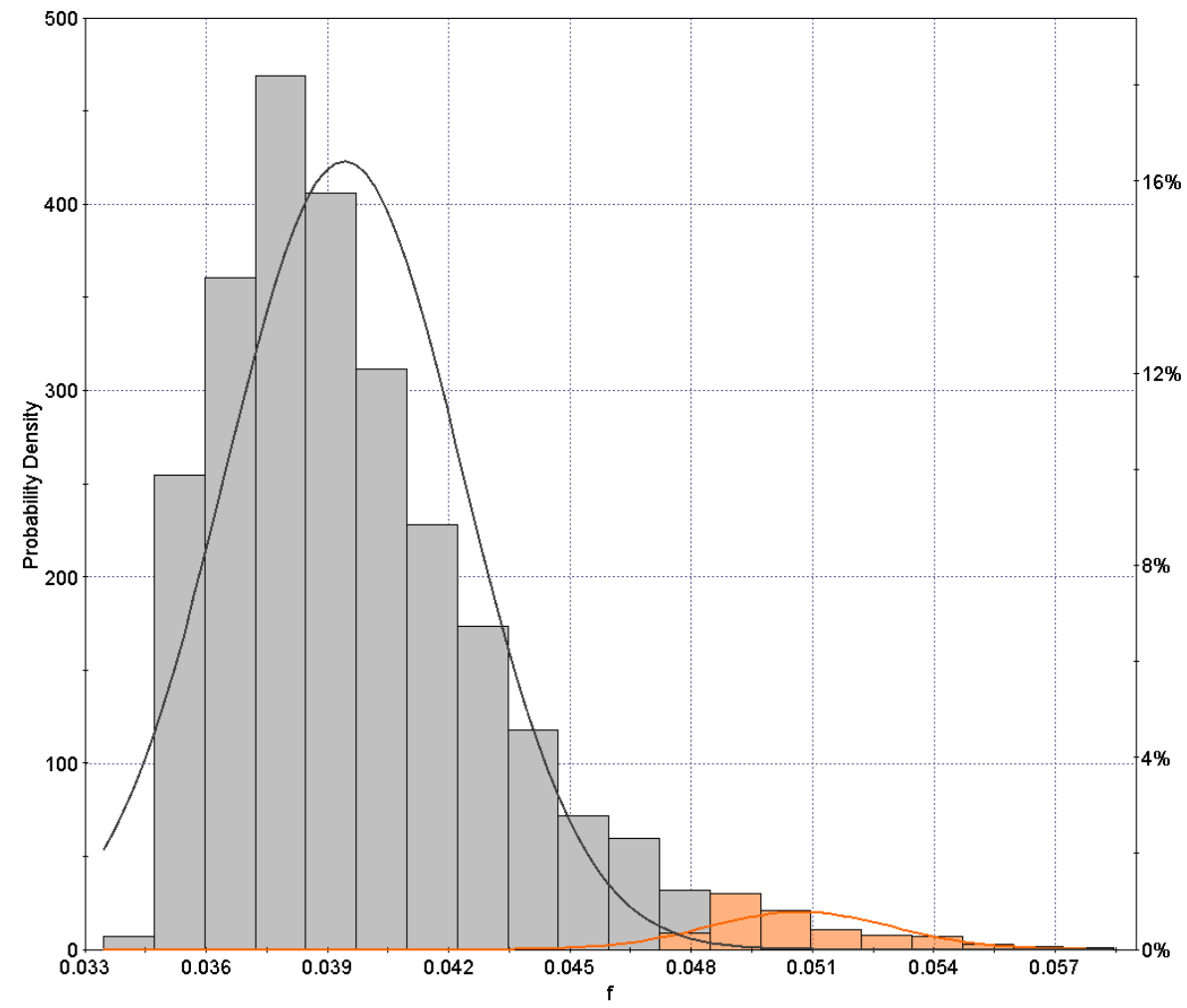

(a)

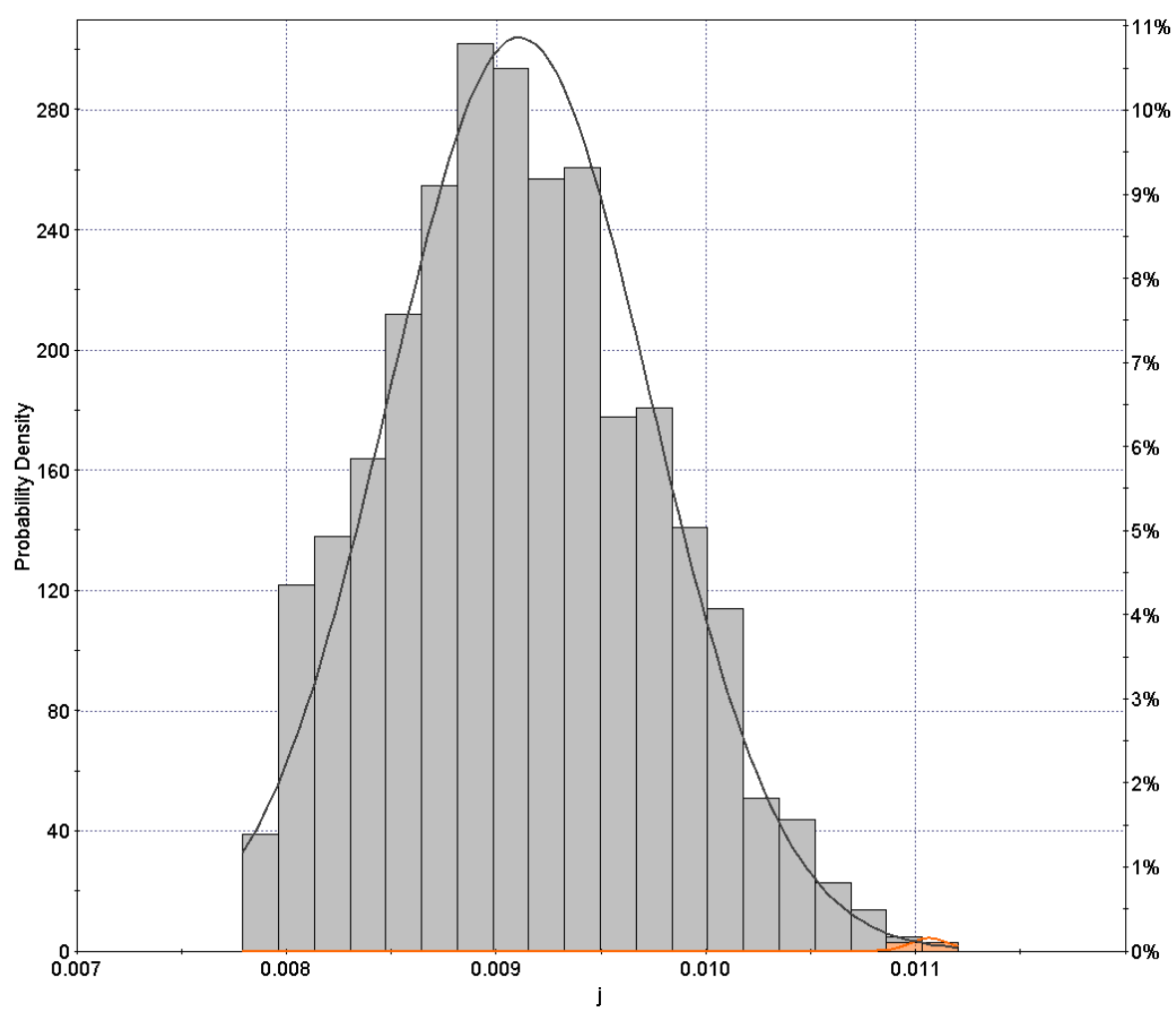

(b)

Figure 48 - Probability density at $\operatorname{Re}=1400$ (a) Friction factor and, (b) Colburn factor. 
As it can be seen, the colorful range in both figures show the "outliers" data found by Equation 82. Thus, these colorful ranges were removed for RSM training (first approach) and put in the test data set, and kept in data set training in a second approach. As the colorful data are not classical outliers, ie, the colorful data are part of the non-linear solution of the enhancement heat transfer, thus, the RSM training results showed that, keeping the "outliers" in training data was the best choice because the training procedure from back-propagation method already recognize the solution patterns, minimizing the error in the test data set.

Although Neural Network was already chosen and discussed at the beginning the Chapter 4, another surrogate models were evaluated, such as: Kriging and Radial Basis Functions (RBF), according to suggestion from Lemouedda et al. (2010). The comparison among them is presented in Appendix B. Both are also implemented on ModeFrontier software. No more detail is described in this section because these surrogates are not the main goal of this work. However, a brief overview was provided below about these models. For Kriging details is recommended the paper by Rasmussen and Williams (2006) and for RBF is indicated the paper by Buhmann (2003).

Kriging is an RSM which can be interpolating or approximating. It is particularly suitable for highly nonlinear responses and for virtual optimization. Kriging is a Bayesian methodology named after professor Daniel Krige, which is the main tool for making previsions employed in geostatistics, eg, for soil permeability, oil and other minerals extraction, etc. The Kriging behavior is controlled by a covariance function, called a variogram, which rules how varies the correlation between the values of the function at different points. A function can be rougher or smoother, can exhibit large or small ranges of variation, can be affected by a certain amount of noise, and all these features can be resumed in a variogram model.

There are several variogram types:

1. Gaussian variogram is infinitely many times differentiable. This should always be the first choice. However the resulting meta-model could result much more regular than the original data (over-smoothing). Moreover, the method is likely to fail the training for large datasets or with large variations in output. In that 
case it is possible to increase the amount of noise, increasing also the smoothing effect, which should be carefully considered.

2. Exponential variogram is the most rough, ie, they are not required to be differentiable, they are only continuous. Thus, this variogram can be used as a failsafe strategy, because the meta-model can be quite irregular, but the training is likely to converge without problems also on quite large datasets and for irregular responses.

3. Matern variogram have an intermediate smoothness between Gaussians and Exponential. Matern 5/2 are twice differentiable, while Matern 3/2 are once differentiable. So, as a general rule, if Gaussian fails, one should try Matern $5 / 2$ (to get a twice differentiable model) as a second choice and Matern 3/2 (to get a differentiable model) as a third choice.

4. Rational Quadratic Rational family of variogram are suitable for modeling functions with non uniform behavior over the design space, ie, sudden variations over some regions and slower over other regions. The meta-models produced are infinitely many times differentiable (very smooth), so they could be difficult to train or produce responses more regular than the data.

The variogram range of the covariance function corresponds to a characteristic scale of the problem. If the distance between two points is larger than the range the corresponding outcomes should not influence each other (completely uncorrelated). Range is inversely related to the number of oscillations of the function. Small ranges mean sudden variations, while large ranges mean very regular trends, with very few oscillations. Summarizing, the general rule in auto-fitting, a Kriging meta-model on a given dataset should be attempted in following sequence: Gaussians, Matern 5/2, Matern 3/2, Rational Q 0.5, Rational Q 2.0 and Exponential.

Radial Basis Functions (RBF) is a powerful tool for multivariate scattered data interpolation. Scattered data means that the training points not need to be sampled on a regular grid: in fact $\mathrm{RBF}$ is a proper meshless method. Since RBF are 
interpolant response surfaces they pass exactly through training points. Two different RBF are available in ModeFrontier: Duchon's Polyharmonic Splines and Hardy's MultiQuadrics, which results are reported here. Furthermore a fully automatic scaling policy is implemented, based on the minimization of the mean leave-one-out error. The scaling parameter determines the shape of the radial function, and in general its value is problem-specific and can be related to the data spatial density: the automatic scaling policy is a suitable method for finding its optimum value. The leave-one-out methodology is an effective system for checking the goodness of an interpolant response (in fact in RBF framework there is a convenient way for computing the rootmean-square). 


\section{Results and Discussions}

This chapter was structured to separately analyze the $R e=250$ and $\operatorname{Re}=1400$. After that, it is presented some qualitative differences between the better results found through the optimization process for each objective functions. Results discussed herein are faced to main researches found in open literature such as: Lemouedda et al. (2010), Pesteei et al. (2005) and Torii et al. (2002).

\subsection{Main and Interaction effects methodology}

A preliminary analysis of the main and interaction effects of input variables on Colburn and Friction factors was conducted by Factor Analysis Methodology, which is an exploratory data evaluation that simultaneously investigates the effect of multiple factors. Main effect is defined as the independent variable impact on a dependent variable averaging across the levels of any other independent variables. Interaction effect is observed whenever the effect of one independent variable depends on the level of the other, Forrester et al. (2008).

Regarding main and interaction effects, consider $D$ the set of numerical points. Let $\mathrm{x}$ be the vector of independent variables with $x_{i} \in\left[x_{i}^{\text {lb }}, x_{i}^{\text {up }}\right], i=1, \ldots, N$ and $y$ be the dependent variable, where "Ib" is the lower boundary and "up" is the upper boundary. It is define $D_{j}^{+}=\left\{x \in D \mid x_{j}>\frac{x_{j}^{u b}-x_{j}^{b b}}{2}\right\}$ and $D_{j}^{-}=\left\{x \in D \mid x_{j}<\frac{x_{j}^{u b}-x_{j}^{b b}}{2}\right\}$, then the main effect of $x_{j}$ is:

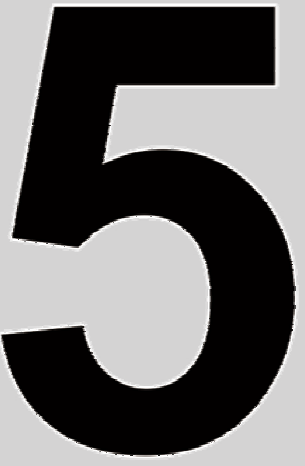




$$
\operatorname{Eff}\left(x_{j}\right)=\frac{\sum x \in D_{j}^{+} y(x)}{\operatorname{card}\left(D_{j}^{+}\right)}-\frac{\sum x \in D_{j}^{-} y(x)}{\operatorname{card}\left(D_{j}^{-}\right)}
$$

where "card( )" denotes the cardinality of the set, ie, is a measure of the number of elements of the set.

The interaction effects can be defined in an analogue way applying the single rules for multiplication to the subsets of $D$. For example, to compute the interaction effect between $x_{1}$ and $x_{2}$ it is compute:

$$
\begin{gathered}
\mathrm{D}_{12}^{+}=\left\{\mathrm{x} \in \mathrm{D}_{1}^{+} \cap \mathrm{D}_{2}^{+}\right\} \cup\left\{\mathrm{x} \in \mathrm{D}_{1}^{-} \cap \mathrm{D}_{2}^{-}\right\} \\
\mathrm{D}_{12}^{-}=\left\{\mathrm{x} \in \mathrm{D}_{1}^{+} \cap \mathrm{D}_{2}^{-}\right\} \cup\left\{\mathrm{x} \in \mathrm{D}_{1}^{-} \cap \mathrm{D}_{2}^{+}\right\} \\
\operatorname{Eff}\left(\mathrm{x}_{1} * \mathrm{x}_{2}\right)=\frac{\sum \mathrm{x} \in \mathrm{D}_{12}^{+} \mathrm{y}(\mathrm{x})}{\operatorname{card}\left(\mathrm{D}_{12}^{+}\right)}-\frac{\sum \mathrm{x} \in \mathrm{D}_{12}^{-} \mathrm{y}(\mathrm{x})}{\operatorname{card}\left(\mathrm{D}_{12}^{-}\right)}
\end{gathered}
$$

This methodology is already implemented in ModeFrontier software and the brief procedure reported above is presented only for reference. More details could be found in NIST/SEMATECH, 2011. For both Reynolds number, similar analysis were made. There are others approaches to analyze either numerical or experimental data set, such as ANOVA. However, Factor Analysis Methodology showed results that corroborates with pattern study, according to the next sections in this chapter.

\subsection{Main and Interaction effects results}

Figure 49 and Figure 50 show the ranking of the contribution ratio for each input variable on Colburn and Friction factor, respectively, beyond the accumulative percentage of the main/interaction effects; input variable impact on factors can be positive (green bar) or negative (red bar). 


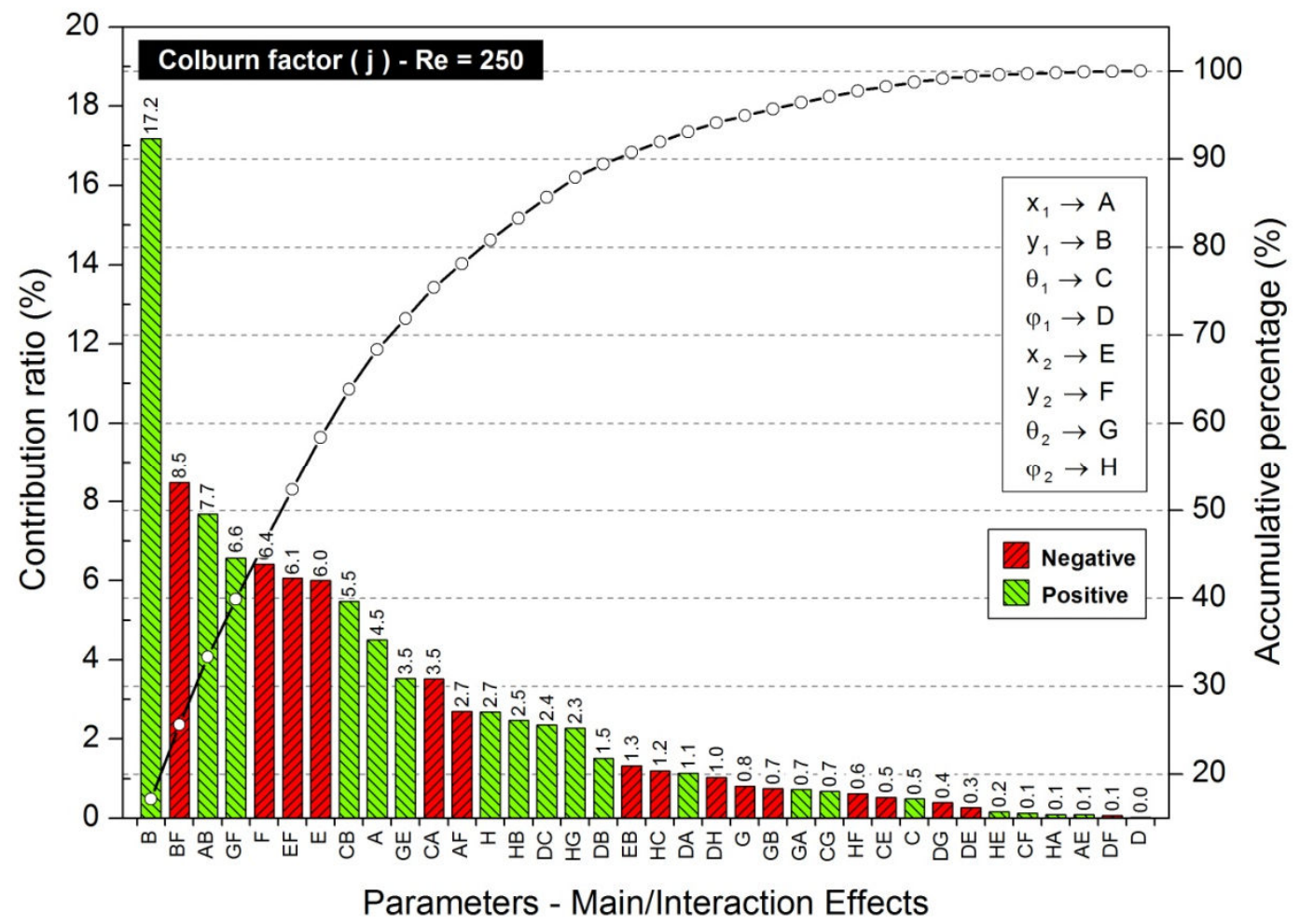

Figure 49 - Contribution ratio of the main/interaction effect for Colburn factor (j) at $\operatorname{Re}=250$.

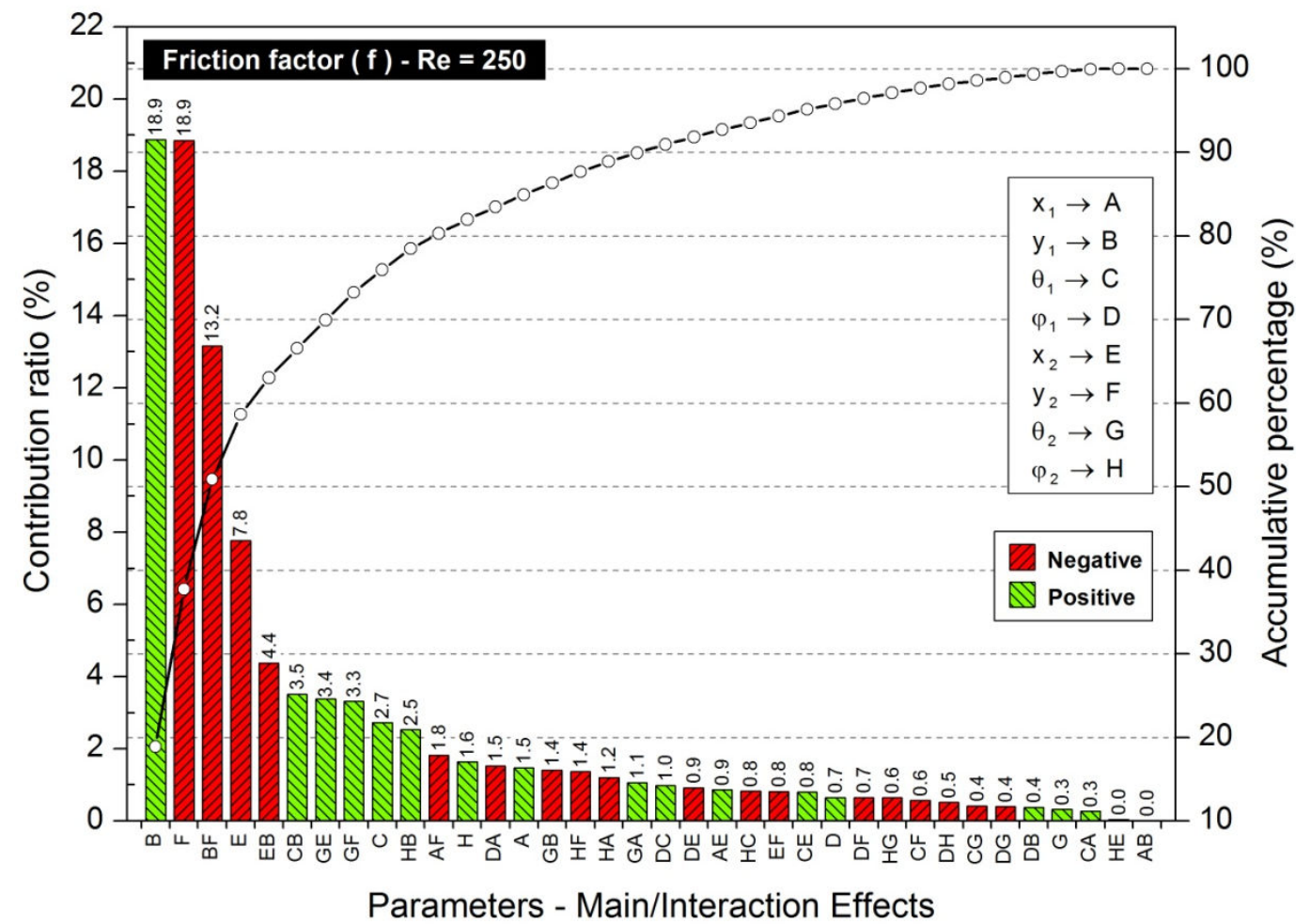

Figure 50 - Contribution ratio of the main/interaction effect for Friction factor (f) at $R e=250$. 
The major conclusions from Figure 49 and Figure 50 are:

- Impact of the position and angles of the $\mathrm{VG}_{2}$ on $\mathrm{j}$ and $\mathrm{f}$ are not symmetric with the $V_{G_{1}}$. Preview researches found in open literature always reported the symmetry between VGs for the first and second tubes;

- VGs configurations (positions and angles) impact on $\mathrm{j}$ and $\mathrm{f}$ in different level. For example, the interaction $\mathrm{x}_{1} \mathrm{y}_{1}$ is an important interaction for Colburn factor but is negligible for Friction factor;

- There is a strong interaction between $V G_{1}$ and $V G_{2}$, especially for $y_{1} y_{2}$, which is supported considering an important feature of the longitudinal vortex that is persistent downstream;

- Impact of the main effect $\varphi_{1}$ on Colburn and Friction factor is small. Effect of the attack angle $\theta_{1}$ is also small on Colburn factor but is slightly important in the variance on Friction factor. However, the interaction $\varphi_{1} \theta_{1}$ is more relevant on Colburn factor. For $V G_{2}$ the main effect $\varphi_{2}$ is more important than $\theta_{2}$ and $\varphi_{2} \theta_{2}$ for both $j$ and $f$. Rolling angles were proposed herein and none researches in open literature were found taking this into account. Thus, rolling angles should be considered in future researches when VG is studied;

- Main effect and all interaction levels of $y_{1}$ cause the highest variation on Colburn factor, $45 \%$, and for Friction factor achieved $44 \%$;

- Interaction between $\theta_{2}$ and $x_{2}$ or $y_{2}$ are much more relevant than considering $\varphi_{2}$ on both $\mathrm{j}$ and $\mathrm{f}$. This same behavior is observed between either $\theta_{1}$ and $\mathrm{x}_{1}$ or $y_{1}$ on $j$. For $f$, the interaction $x_{1} \varphi_{1}$ is more relevant than $x_{1} \theta_{1}$, for example. These results are important to confirm the raised initial hypothesis that the vortex generator positions and angles should be independent for each tube to reach, as possible as, the maximum thermal-hydraulic performance;

- $\mathrm{y}_{1}, \mathrm{y}_{1} \mathrm{y}_{2}$, and $\mathrm{x}_{1} \mathrm{y}_{1}$ match around $33.4 \%$ of variance on Colburn factor and $\mathrm{y}_{1}, \mathrm{y}_{2}$ and $\mathrm{y}_{1} \mathrm{y}_{2}$ match around $51 \%$ on Friction factor.

This same analysis is made for $\operatorname{Re}=1400$ and is discussed below. 


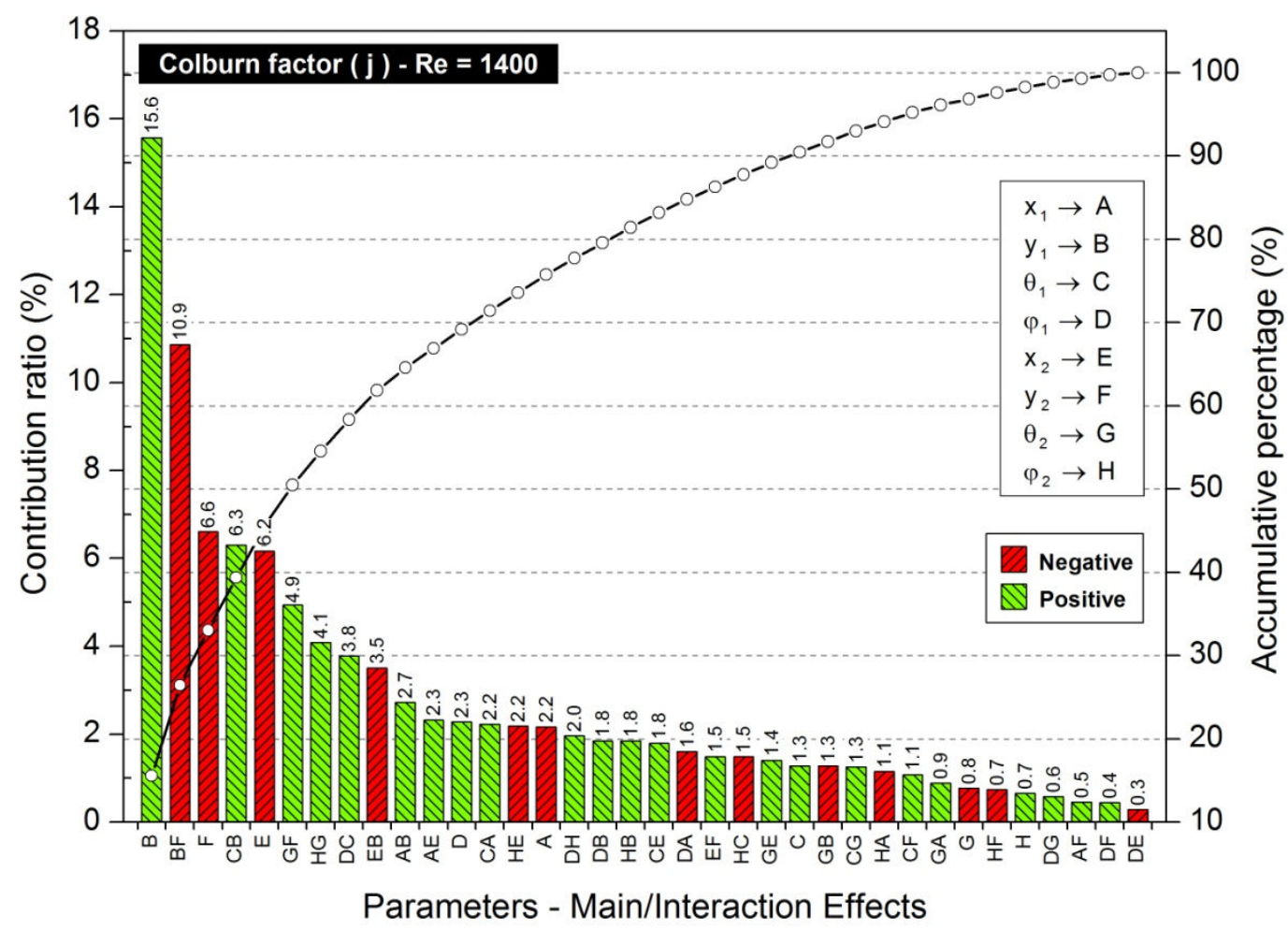

Figure 51 - Contribution ratio of the main/interaction effect for Colburn factor (j) at $\operatorname{Re}=1400$

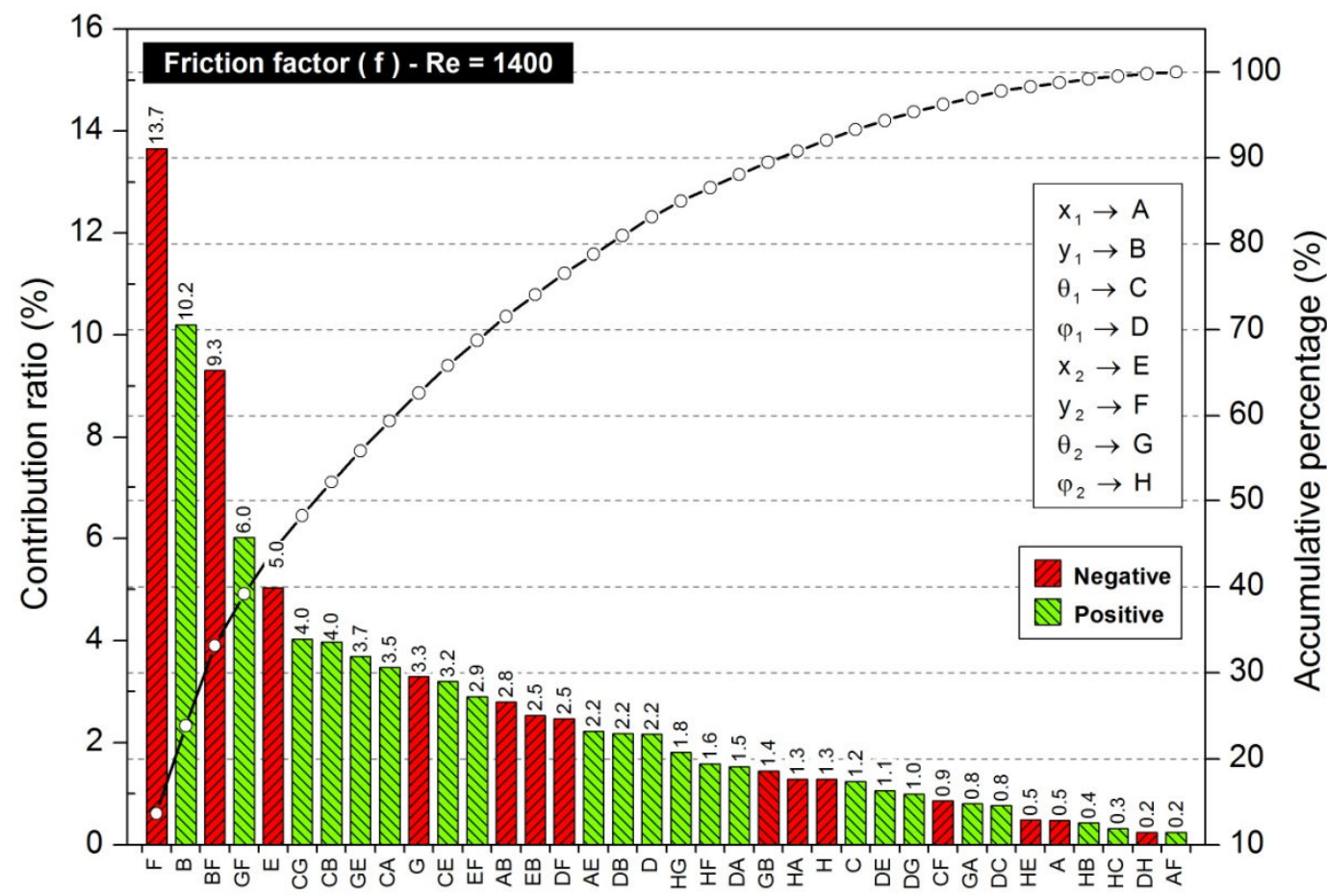

Parameters - Main/Interaction Effects

Figure 52 - Contribution ratio of the main/interaction effect for Friction factor (f) at $\operatorname{Re}=1400$. 
For $\operatorname{Re}=1400$, from Figure 51 and Figure 52 the major conclusions are:

- Impact on Colburn and Friction factors of the $\mathrm{VG}_{2}$ position and angles are not symmetric with the $\mathrm{VG}_{1}$;

- VGs configurations (positions and angles) impact on $\mathrm{j}$ and $\mathrm{f}$ in different level. For example, the interaction $\theta_{1} \theta_{2}$ is an important interaction for Friction factor but is slightly negligible for Colburn factor;

- There is a great interaction between $V G_{1}$ and $V G_{2}$, especially for $y_{1} y_{2}$. Again, this assessment is supported considering that the longitudinal vortex is persistent downstream;

- Considering the $\mathrm{VG}_{1}$, the interaction $\varphi_{1} \theta_{1}$ is more relevant than the main effect $\varphi_{1}$ and $\theta_{1}$ on Colburn factor; however, on Friction factor the main effects are more important than interaction effect. For Colburn factor considering $V G_{2}$, the interaction $\varphi_{2} \theta_{2}$ is significantly more important than the main effect $\varphi_{2}$ and $\theta_{2}$; however, for Friction factor the main effect $\theta_{2}$ is more vital than $\varphi_{2} \theta_{2}$ and $\varphi_{2}$. Therefore, even as concluded for $\operatorname{Re}=250$, rolling angles should be considered in future researches as a new input variable in augmentation heat transfer with longitudinal vortex generator;

- Main effect and all interaction levels of $y_{1}$, cause the highest variation on Colburn factor, 44\%. For Friction factor, $y_{2}$ (main and all interaction effects) causes the highest variation reaching around $37 \%$;

- In direction $y, y_{1} \theta_{1}$ and $y_{2} \theta_{2}$ are strongest relation for both Colburn and Friction factor. In direction $\mathrm{x}, \mathrm{x}_{1} \theta_{1}$ and $\mathrm{x}_{2} \theta_{2}$ are important only for Friction factor;

- $\mathrm{y}_{1}, \mathrm{y}_{1} \mathrm{y}_{2}, \mathrm{y}_{2}$ and $\mathrm{y}_{1} \theta_{1}$ match around $39.4 \%$ of variance on Colburn factor and $\mathrm{y}_{2}$, $\mathrm{y}_{1}, \mathrm{y}_{1} \mathrm{y}_{2}$ and $\mathrm{y}_{2} \theta_{2}$ match around $39.2 \%$ on Friction factor.

These outlined conclusions showed that the positions ( $x$ and $y$ ) are more impactful on Colburn and Friction factors than angles $(\theta$ and $\varphi)$. However, the interaction among them becomes very important. Following, considering Figure 53 and Figure 54, it is 
made a qualitative comparison of the main and interaction effect between $\operatorname{Re}=250$ and $\operatorname{Re}=1400$ for both Colburn and Friction factor.

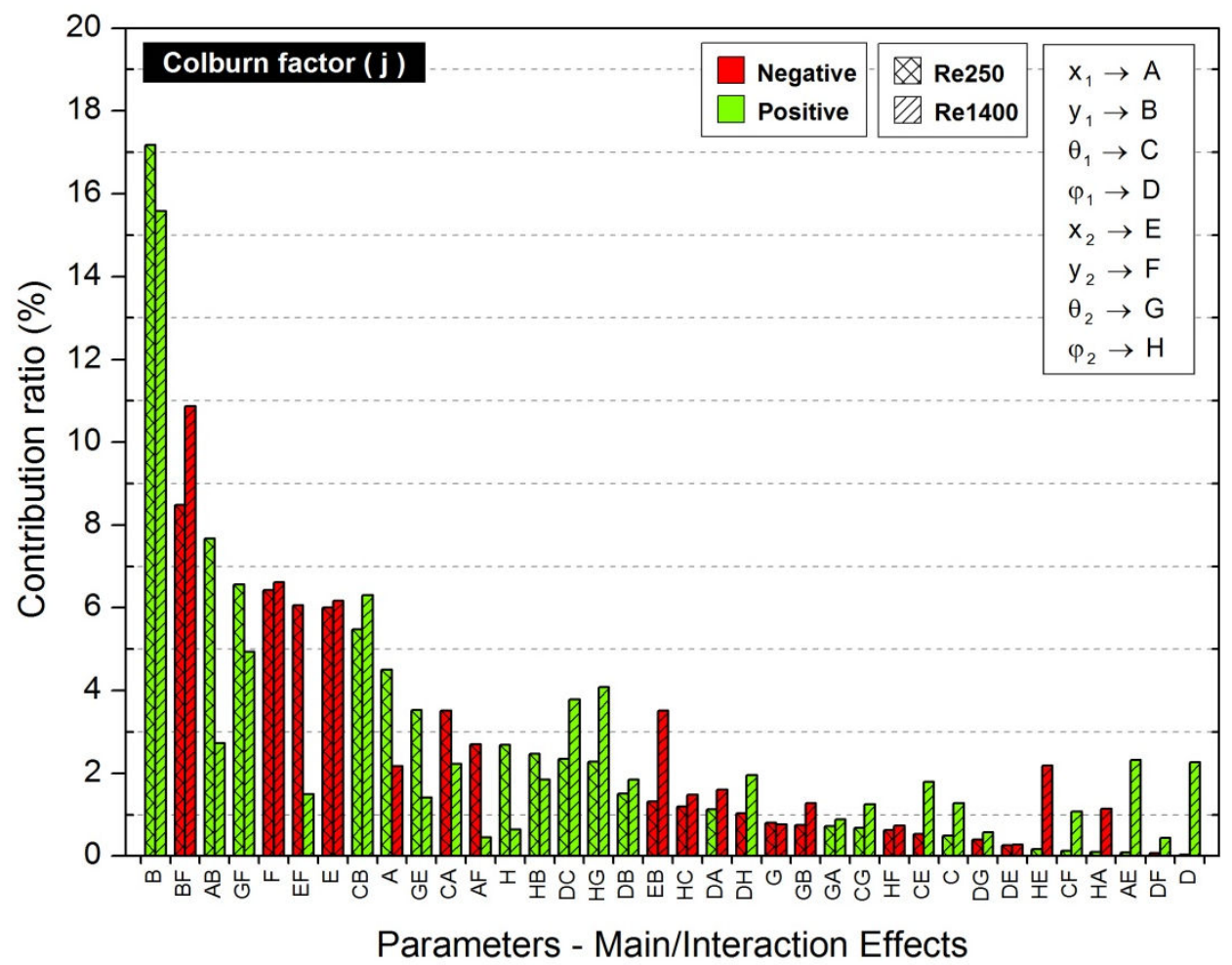

Figure 53 - Comparison of the contribution ratio of main/interaction effect for Colburn factor (j).

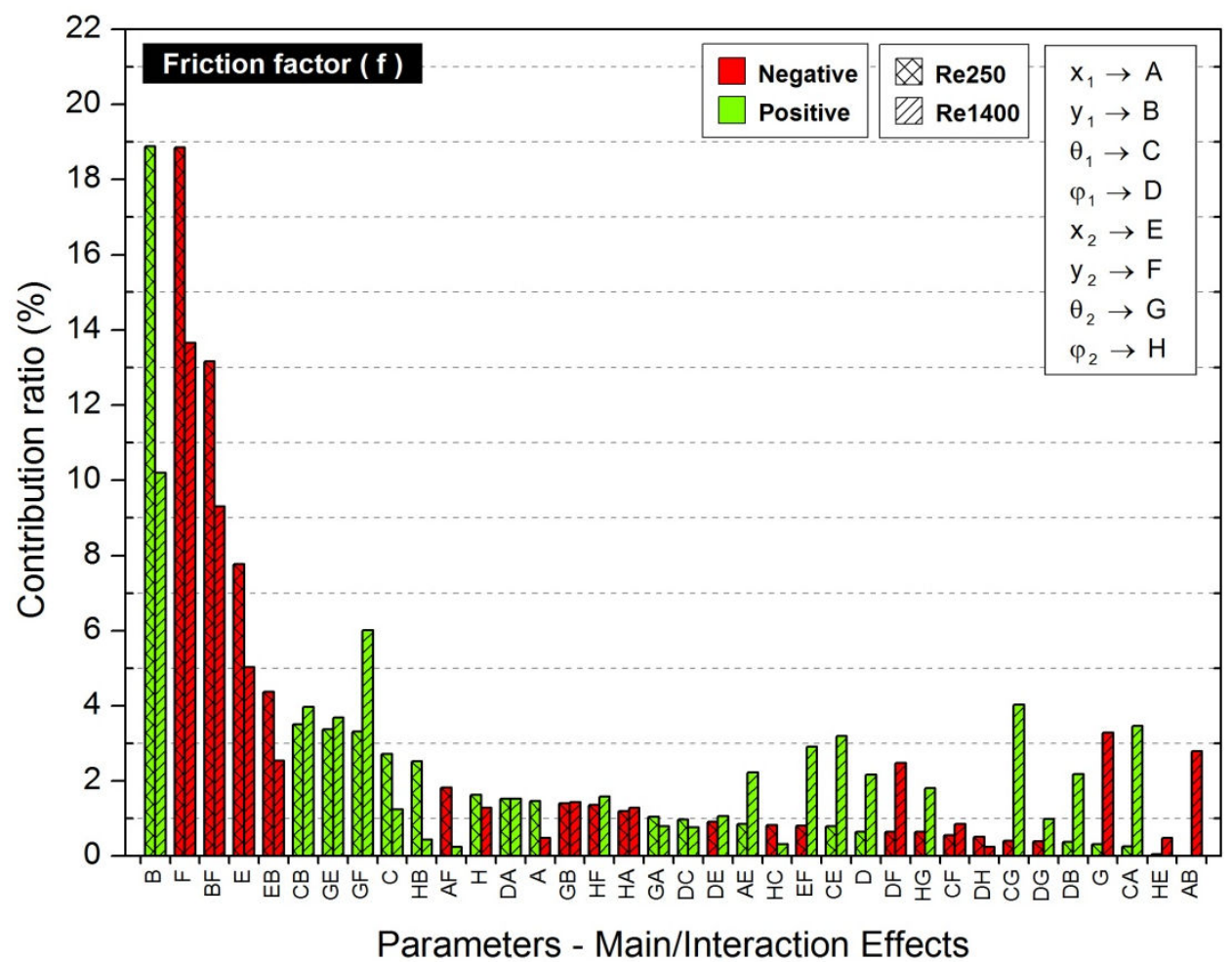

Figure 54 - Comparison of the contribution ratio of main/interaction effect for Friction factor (f). 
One of the raised initial hypotheses in this thesis is that the vortex generator positions and angles should be independent. This question was already answered. The next question naturally raised is about the impact of the input variables on Colburn and Friction factor for different Reynolds number. In the open literature, there is no significant difference in region where VG is placed and the angle range for different Reynolds number. Studies focused in small changes in attack angle and position for both high and low Reynolds number. However, in Figure 53 and Figure 54, Colburn and Friction factor are changed differently for each main/interaction effects. For example, on Colburn factor the effects $\varphi_{1}, x_{1} x_{2}$ and $x_{2} \varphi_{2}$ cause some variance for $\operatorname{Re}=1400$ and nearly null for $\operatorname{Re}=250$. For Friction factor, this same behavior is verified considering the effects $\theta_{1} \theta_{2}, \theta_{2}, x_{1} \theta_{1}$ and $x_{1} y_{1}$. These results corroborate with the initial hypotheses and indicate the need of an optimization process for each Reynolds number. Moreover, some input variables cause opposite effect on Colburn and Friction factors; for example, for Colburn factor, $\mathrm{x}_{2} \mathrm{y}_{2}$ decreases for $\operatorname{Re}=250$ whereas increases for $\operatorname{Re}=1400$. It is also observed for other effects, such as $x_{1}$ and $x_{1} \theta_{1}$. Similarly, for Friction factor is also verified especially for the effects $\varphi_{2}, \mathrm{y}_{2} \varphi_{2}$ and $\mathrm{x}_{2} \mathrm{y}_{2}$.

Overall, the conclusions made in this section rationalize and confirm the initial hypotheses of this thesis regarding the independence of the vortex generator. Moreover, the main and interaction effects of the input variables differently impact on Colburn and Friction factors for each Reynolds number, indicating the need of an individual optimization process.

The discussion outlined do not point out about the thermal-hydraulic phenomenon presented in Chapter 1 and 2. Thus, the results explored in the next sections focus in the flow pattern study among the present work (optimized configuration by RSM methodology and direct optimization) and the major referenced researches in open literature. 


\subsection{Flow pattern}

Flow pattern study presented herein is focused on identification of the vortex structures, span-average temperature distribution, span-average Colburn factor and pressure profile for each vortex generator arrangement. Results reported herein are identified as RSM and DO which are the optimal configurations found by Response Surface Methodology (RSM) and Direct Optimization (DO), respectively. These results were faced to main surveys in the open literature: Lei et al (2010), Torii et al. (2002), Lemouedda et al. (2010) and Pesteei et al. (2005). There are peculiarities among these current works, such as: Reynolds range and geometric parameters of the heat exchanger. Therefore, the position of the VG was parameterized as a function of tube diameter and fin length. Moreover, VG arrangement studied by Lemouedda et al. (2010) and Pesteei et al. (2005), positioned on both common-flowdown and common-flow-up were also evaluated.

Table 8 presents the positions and angles for each research studied, according to reference shown in Figure 14. Vortex generator configurations from literature surveys were considered for both Reynolds number $(\operatorname{Re}=250$ and $\operatorname{Re}=1400)$.

Soon, from literature surveys, vortex generator position and angle are different even when the same approach is used, eg, common-flow-up or common-flow-down configurations; both were evaluated. This shows that defining the best vortex generator position and angle remains a major challenge regarding to augmentation heat transfer. Moreover, from optimization results, a significant difference between optimal configurations found for each Reynolds number was observed. Several results in Table 8 restating some previous conclusions, reminding them:

1. Rolling angle $(\varphi)$, input variable proposed herein, actually should be considered by others researchers as an important vortex generator dimensional;

2. Input variable to $\mathrm{VG}_{1}$ and $\mathrm{VG}_{2}$ are not symmetric for optimal configuration.

3. The optimal VG position and angle changes for each Reynolds number. 
Table 8 - Position and angle according to reference adopted in present work.

\begin{tabular}{|c|c|c|c|c|c|c|c|c|c|c|}
\hline \multirow{2}{*}{\multicolumn{3}{|c|}{ Researchers }} & \multicolumn{8}{|c|}{ Input variable } \\
\hline & & & $\mathrm{x}_{1}$ & $\mathrm{y}_{1}$ & $\theta_{1}$ & $\varphi_{1}$ & $\mathrm{x}_{2}$ & $y_{2}$ & $\theta_{2}$ & $\varphi_{2}$ \\
\hline \multicolumn{3}{|c|}{ Lei et al. (2010) } & 12.8 & 4.5 & 340 & 0 & 32.8 & 6.5 & 20 & 0 \\
\hline \multirow{2}{*}{\multicolumn{3}{|c|}{ Lemouedda et al. (2010) - Down }} & 15.5 & 4.7 & 60 & 0 & 35.5 & 6.3 & 300 & 0 \\
\hline \multirow{2}{*}{\multicolumn{3}{|c|}{ Lemouedda et al. (2010) - Up }} & 15.5 & 4.7 & 300 & 0 & 35.5 & 6.3 & 60 & 0 \\
\hline & & & 14.3 & 4.3 & 45 & 0 & 34.3 & 6.7 & 315 & 0 \\
\hline \multicolumn{3}{|c|}{ Pesteei et al. (2005) - Up } & 14.3 & 4.3 & 315 & 0 & 34.3 & 6.7 & 45 & 0 \\
\hline \multicolumn{3}{|c|}{ Torii et al. (2002) } & 11.1 & 5.4 & 345 & 0 & 31.1 & 5.6 & 15 & 0 \\
\hline \multirow{8}{*}{$\begin{array}{l}\text { Present } \\
\text { Work } \\
\operatorname{Re}=250\end{array}$} & \multirow{2}{*}{$\begin{array}{c}\text { JF } \\
\text { (Equation 64) }\end{array}$} & RSM & 2.5 & 2.5 & 69 & -21 & 32.7 & 5.0 & 33 & -3 \\
\hline & & $\mathrm{DO}$ & 2.9 & 2.5 & 68 & -40 & 32.2 & 5.1 & 40 & -15 \\
\hline & \multirow{2}{*}{$\begin{array}{c}\mathrm{JF}^{\frac{1}{3}} \\
\text { (Equation 65) }\end{array}$} & RSM & 9.4 & 6.5 & 322 & 4 & 33.4 & 5.9 & 241 & 17 \\
\hline & & $\mathrm{DO}$ & 10.2 & 6.5 & 318 & -16 & 35.6 & 6.5 & 264 & 11 \\
\hline & \multirow{2}{*}{$\begin{array}{c}\mathrm{j} \\
\text { (Equation 62) }\end{array}$} & RSM & 9.7 & 7.2 & 312 & 1 & 33.7 & 6.1 & 257 & 19 \\
\hline & & $\mathrm{DO}$ & 9.0 & 6.5 & 320 & 3 & 33.4 & 5.7 & 252 & 17 \\
\hline & \multirow{2}{*}{$\begin{array}{c}f \\
\text { (Equation 63) } \\
\end{array}$} & RSM & 15.4 & 2.5 & 176 & -14 & 34.2 & 6.9 & 202 & -2 \\
\hline & & $\mathrm{DO}$ & 15.9 & 2.5 & 347 & 35 & 33.7 & 5.7 & 36 & -14 \\
\hline \multirow{8}{*}{$\begin{array}{l}\text { Present } \\
\text { Work } \\
\operatorname{Re}=1400\end{array}$} & \multirow{2}{*}{$\begin{array}{c}\mathrm{JF} \\
\text { (Equation 64) }\end{array}$} & RSM & 6.1 & 6.9 & 340 & -11 & 33.0 & 5.4 & 36 & -10 \\
\hline & & $\mathrm{DO}$ & 11.1 & 7.4 & 332 & -5 & 35.3 & 6.8 & 148 & -3 \\
\hline & \multirow{2}{*}{$\begin{array}{c}\mathrm{JF}^{\frac{1}{3}} \\
\text { (Equation 65) }\end{array}$} & RSM & 2.7 & 5.7 & 63 & 16 & 25.1 & 3.4 & 43 & -26 \\
\hline & & DO & 7.5 & 7.4 & 328 & 11 & 35.3 & 6.8 & 148 & -3 \\
\hline & \multirow{2}{*}{$\begin{array}{c}\mathrm{j} \\
\text { (Equation 62) }\end{array}$} & RSM & 9.4 & 6.7 & 125 & -3 & 28.8 & 2.7 & 61 & 4 \\
\hline & & $\mathrm{DO}$ & 8.9 & 8.2 & 287 & -20 & 26.3 & 2.6 & 222 & 13 \\
\hline & \multirow{2}{*}{$\begin{array}{c}f \\
\text { (Equation 63) }\end{array}$} & RSM & 2.6 & 2.7 & 299 & 42 & 33.0 & 6.4 & 213 & 2 \\
\hline & & DO & 3.7 & 4.4 & 200 & -44 & 32.1 & 5.1 & 44 & -39 \\
\hline
\end{tabular}

Moreover, RSM and DO results are considerably different and, as can be seen in the next sections, the $D O$ results are higher than $R S M$, especially for $R e=1400$. Also, for each objective function there is a specific optimal vortex generator arrangement; for example, the optimal VG configuration found for the objective function JF is not necessarily optimal for the objective function $\mathrm{JF}^{\frac{1}{3}}$. Thus, for others objective functions proposed either by researchers or by heat exchanger designer, an alone optimization procedure should be conducted for each.

In the next sections the results in Table 8 are discussed considering the objective functions asunder for each Reynolds number. 


\subsubsection{Flow pattern for $\operatorname{Re}=\mathbf{2 5 0}$}

Initially, global results are presented for each objective functions, Figure 55 to Figure 58 , faced to literature researches mentioned in Table 8.

Global results for both approaches adopted herein (RSM and DO) were superior than reported by works in the open literature for all objective functions analyzed. Also, results for DO were higher than RSM approach, although the Forrester et al. (2008) recommendations were met. However, regarding the RSM characteristics, it must be capable of capturing the main behavior of a specific problem, with great ability of generalization. In this case, the flow features in a CHE are known by complexity, eg, in front of the tubes are formed the horseshoe vortexes; behind the tubes there is a wake region with flow recirculation; and flow separation on the tubes is also noted. Considering these complexities and the eight input parameters of the VGs, RSM must be able to provide the satisfactory output function. For the test data defined during the RSM training, the quality indicators were satisfactory, but the RSM quality could be degraded for another test set data. Therefore, some RSM benefits would not be so obvious, considering that, for RSM quality check, different test data would be necessary, accounting many numerical models. Moreover, the initial population applied on Genetic Algorithm could contribute for this difference between DO and RSM results. This verification should be made considering the procedure depicted in Figure 43 per many times. However, a lot of the time would be taken for this action, and this analysis is suggested for a future work.

For JF, Figure 55, the results by present work were slightly superior than reported by Torii et al. (2002). Arrangements by DO and RSM are very similar. However, in Figure 59a, the position and angle among the present work and the literature researches are different especially for the $V_{G_{1}}$. Another important feature, and already related by literature surveys, is related to the great difficult to achieve JF $>1$ for low Reynolds number; VG arrangements by present work and Torii et al. (2002) achieved this. Also, common-flow-up seems to be the better choice than commonflow-down configuration, according to assessments by Torii et al. (2002). 
For $\mathrm{JF}^{\frac{1}{3}}$, the result changed a lot faced to JF. Firstly, evaluating only literature works, VG configuration by Lemouedda et al. (2010) "Down" presents the better performance than Torii et al. (2002). Evaluating $\mathrm{JF}^{\frac{1}{3}}$, the heat transfer is a priority compared with the pressure loss penalty. Again, the configuration found by present work was completely different than reported by Lemouedda et al. (2010), Figure 59b, and in Figure 56, the global results were considerably higher than in the open literature researches. Although the global results of the RSM and DO approaches were similar, the VG position and angles were significantly different. This shows that there is more than one $\mathrm{VG}$ arrangement to achieve suitable $\mathrm{JF}^{\frac{1}{3}}$. These results demonstrate that arrangements from optimizer are local optimum and not global optimum.

Depending on the objective of the industrial application, the main goal of the heat exchanger could be to release the maximum heat transfer rate, independently of the pressure loss penalty, for example, for steady application. Figure 57 presents the global results considering the maximum Colburn factor $\mathrm{j}$. RSM and DO approaches achieved better results than those from literature surveys. These results from optimization procedure were significantly higher than current researches, which confirm the great advantage of the methodology proposed herein. Figure 59b and Figure 59c indicate that the optimization procedure (for both RSM and DO) conducted the VG arrangement to similar positions and angles for objective function $j$ and $\mathrm{JF}^{\frac{1}{3}}$, respectively. These results corroborate with the assessment concluded for $\mathrm{JF}^{\frac{1}{3}}$, which the heat transfer rate is a priority compared to the pressure loss penalty. The same way as for $\mathrm{JF}^{\frac{1}{3}}$, among the works in open literature, Lemouedda et al. (2010) "Down" present better performance to maximum heat transfer. Also, comparing only the literature works, common-flow-down configuration was better choice when the heat transfer rate is the main goal; obviously, the pressure penalty to this was higher than common-flow-up arrangement.

The procedure applied to pressure loss minimization in heat exchanger could not make sense keeping in mind that the objective of the heat exchanger is to produce heat transfer between fluids. However, the main goal of this analysis is to identify the vortex structures or interesting features, which could generate reduction in pressure 
loss and, perhaps, extend these features for other analysis. A detailed discussion about this objective function is made later in this sections. However, in Figure 58, the optimization procedure found a VG arrangement, which was able to reduce the pressure loss. A coarse analysis considering the configurations from RSM and DO, Figure $59 \mathrm{~d}$, gives evidences that the $V \mathrm{G}_{1}$ is not important to reduce the pressure loss, considering that the $\mathrm{VG}_{1}$ is placed almost behind the tube, immersed in wake region. This result is later explored in this section. For this objective function, common-flow-up configuration was a better choice than common-flow-down configuration. Among the current researches, Lei et al. (2010) showed the best results, which this VG arrangement is very similar to Torii et al. (2002).

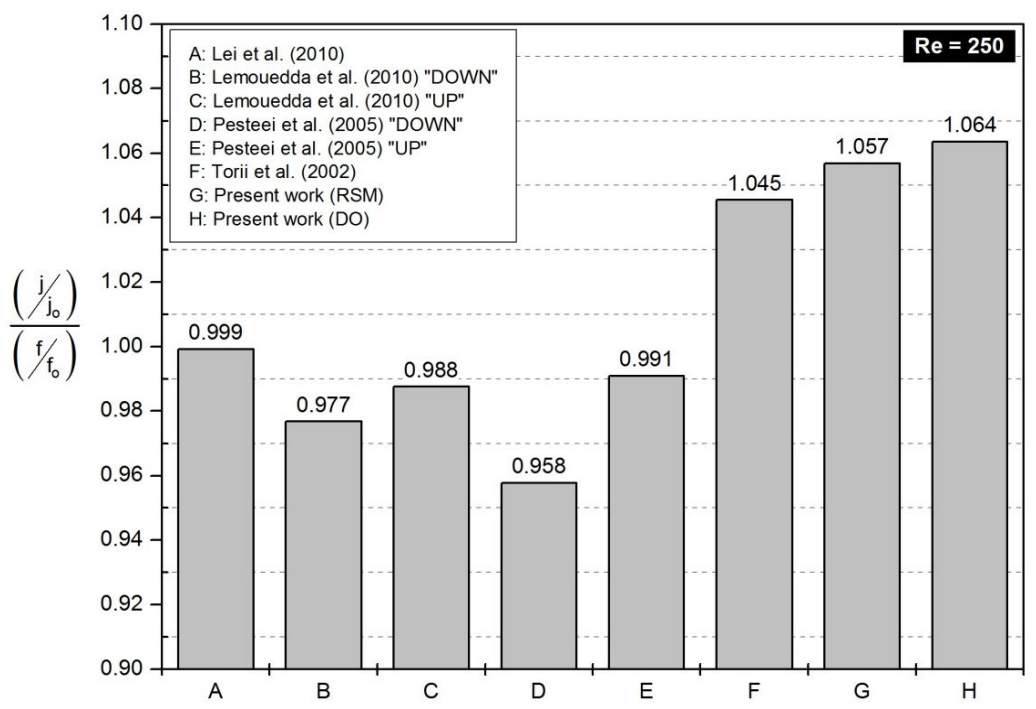

Figure 55 - Global results for JF objective function.

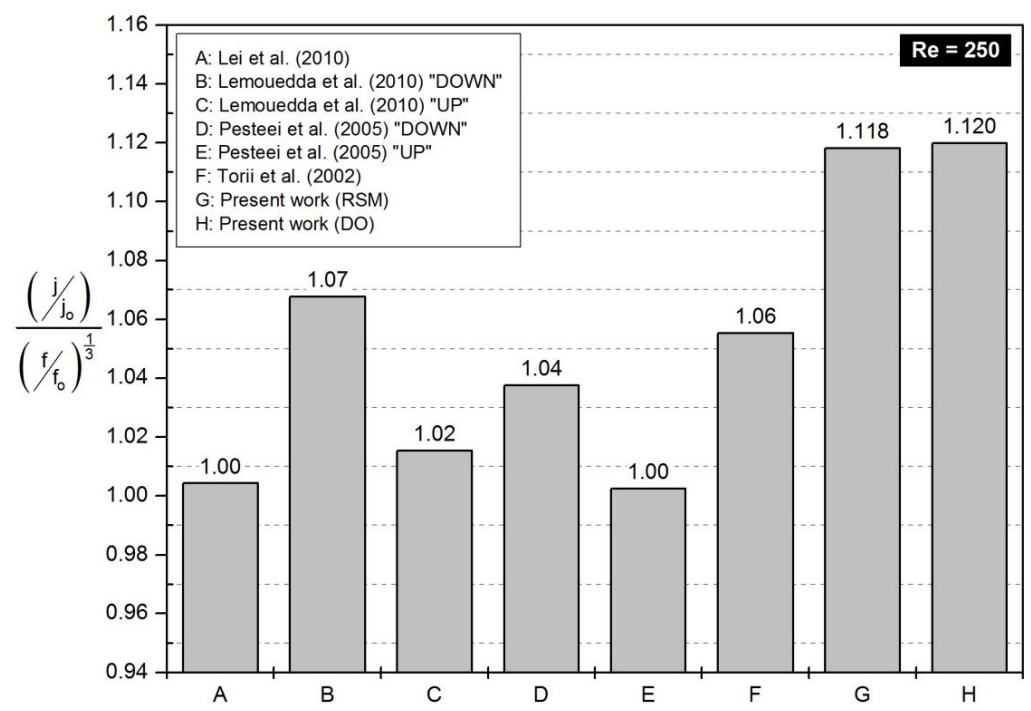

Figure 56 - Global results for $\mathrm{JF}^{\frac{1}{3}}$ objective function. 


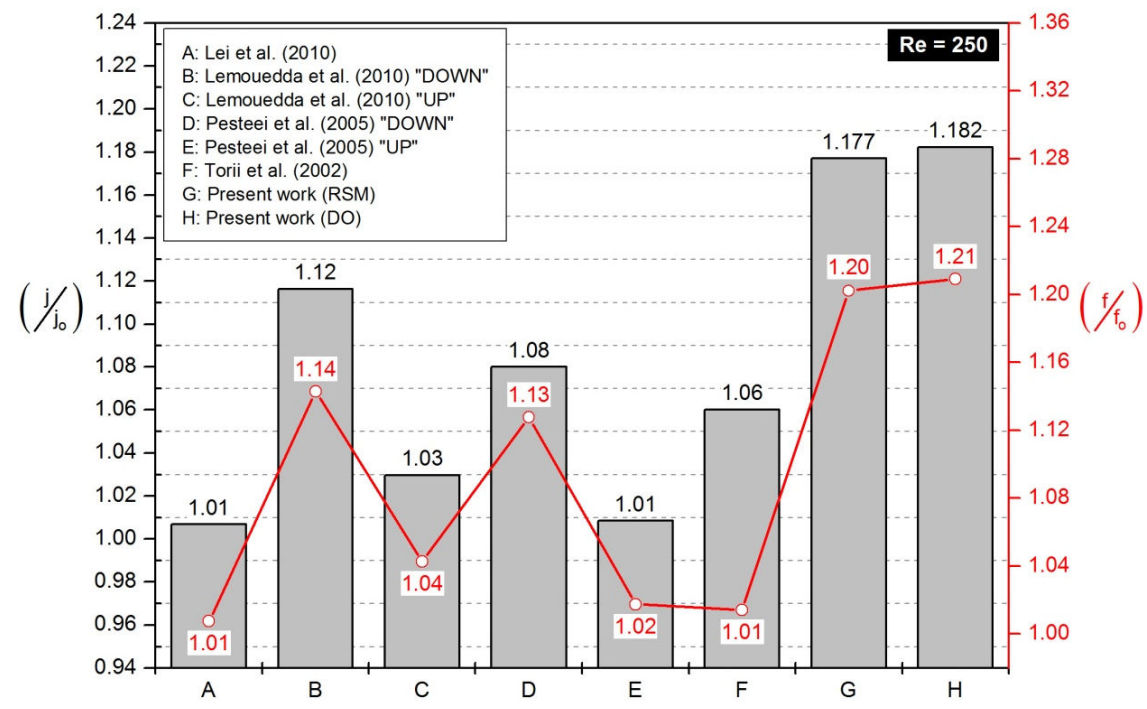

Figure 57 - Global results for j objective function with correspondent drop pressure penalty.

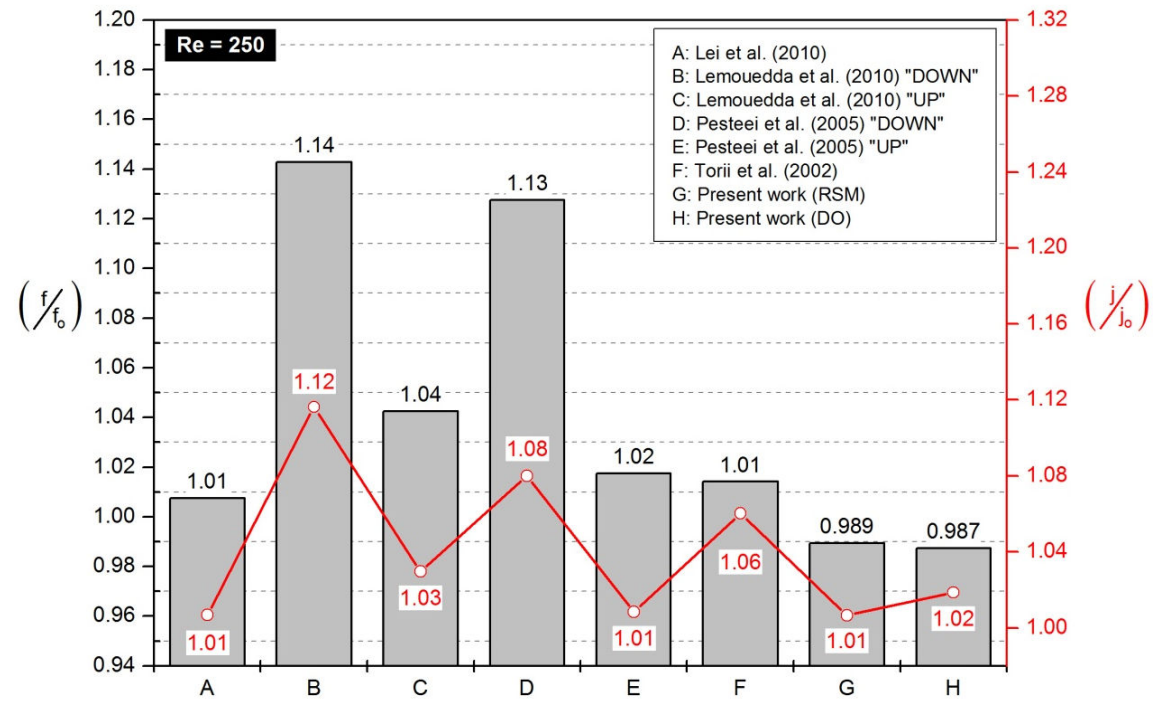

Figure 58 - Global results for $f$ objective function with correspondent augmentation heat transfer.

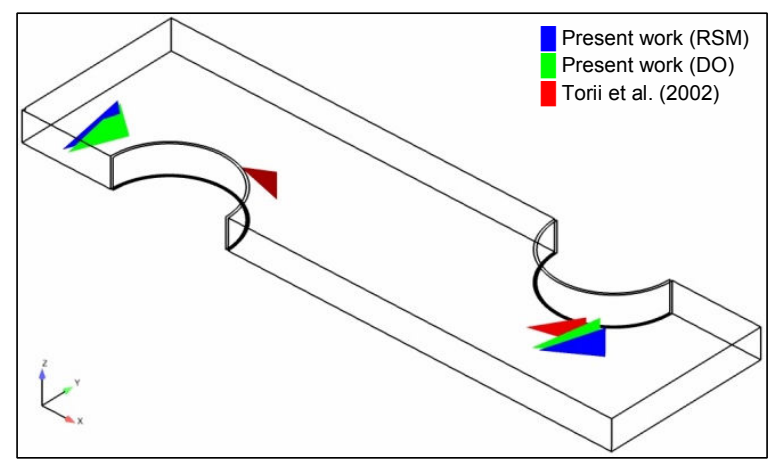

(a) JF

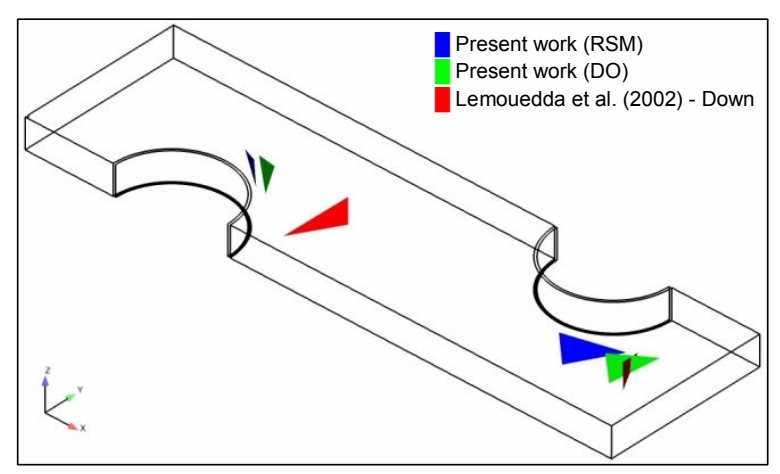

(b) $\mathrm{JF}^{\frac{1}{3}}$ 


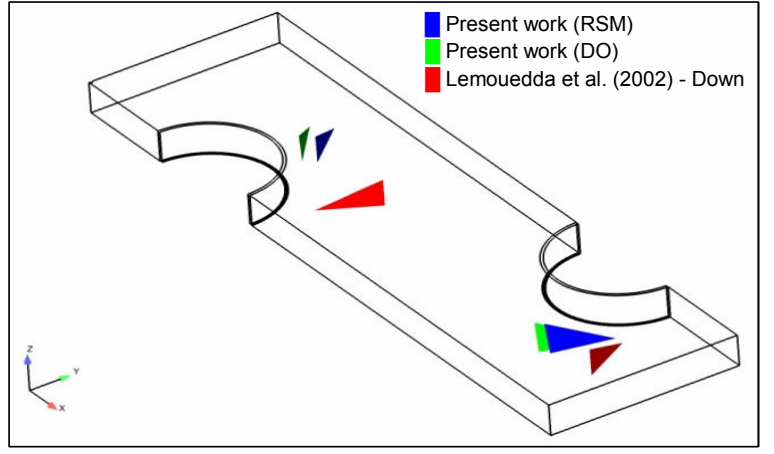

(c) Colburn factor, j

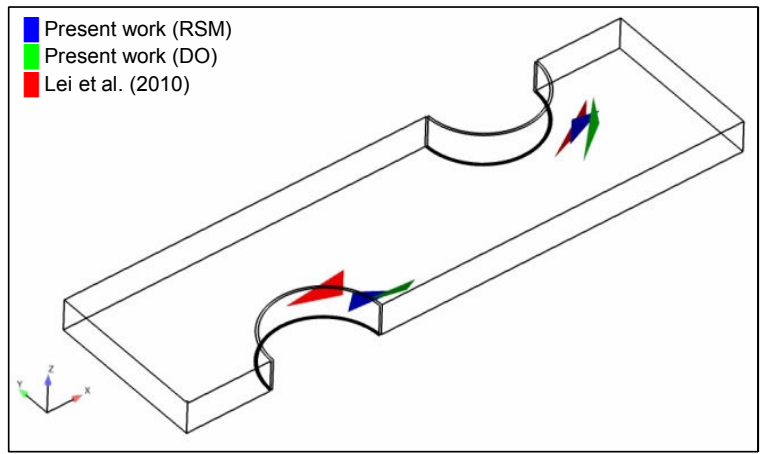

(d) Friction factor, $f$

Figure 59 - Overview of the position and angle for the best results from Figure 55 to Figure 58.

The global results outlined are very important to higher level decision. However, understanding where and how the key phenomena occur is essential to increase the knowledge in augmentation heat transfer and, if possible, prolong its effects. Figure 60 to Figure 62 present the distribution of span-average Colburn factor along the fin length of the heat exchanger with and without vortex generator for each objective function, except to objective function $\mathrm{f}$, which does not make sense evaluate Colburn factor when the objective is to minimize the Friction factor. In order to allow a clean analysis, only the best results from Figure 55 to Figure 57 were plotted. Reminding, Fin-tube described at graphic legend relates to heat exchanger without VG.

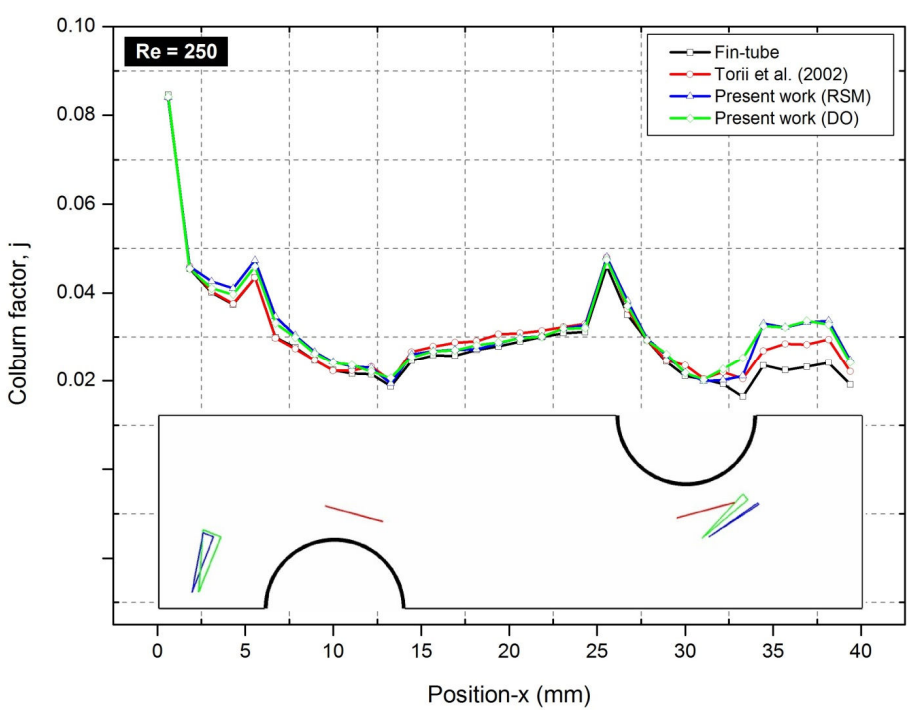

Figure 60 - Span-average Colburn factor (j) for the best results (Figure 55) for objective function JF. 


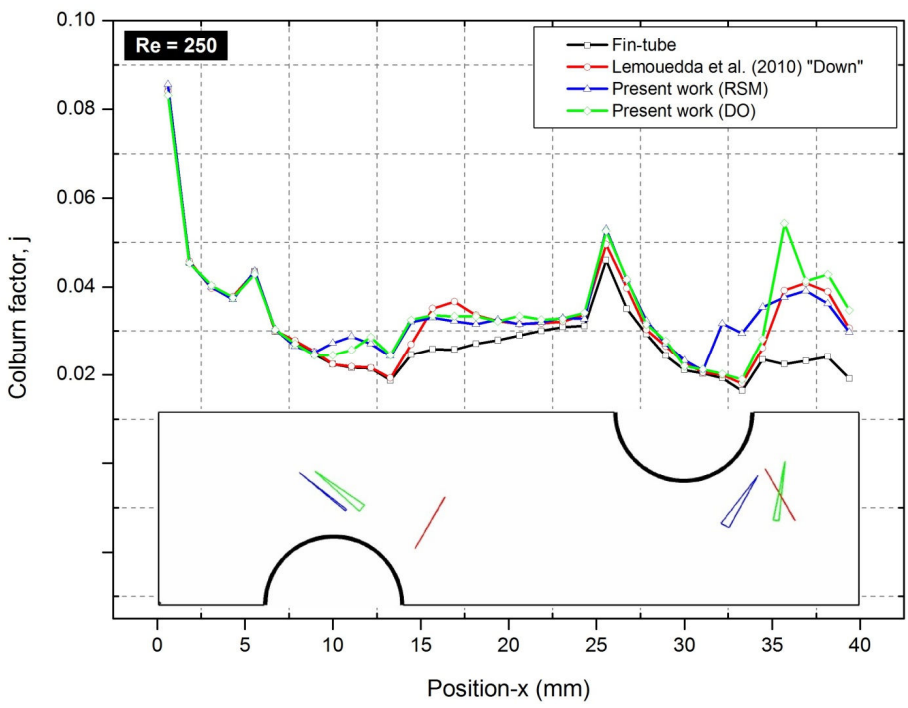

Figure 61 - Span-average Colburn factor (j) for the best results (Figure 56) for objective function $\mathrm{JF}^{\frac{1}{3}}$.

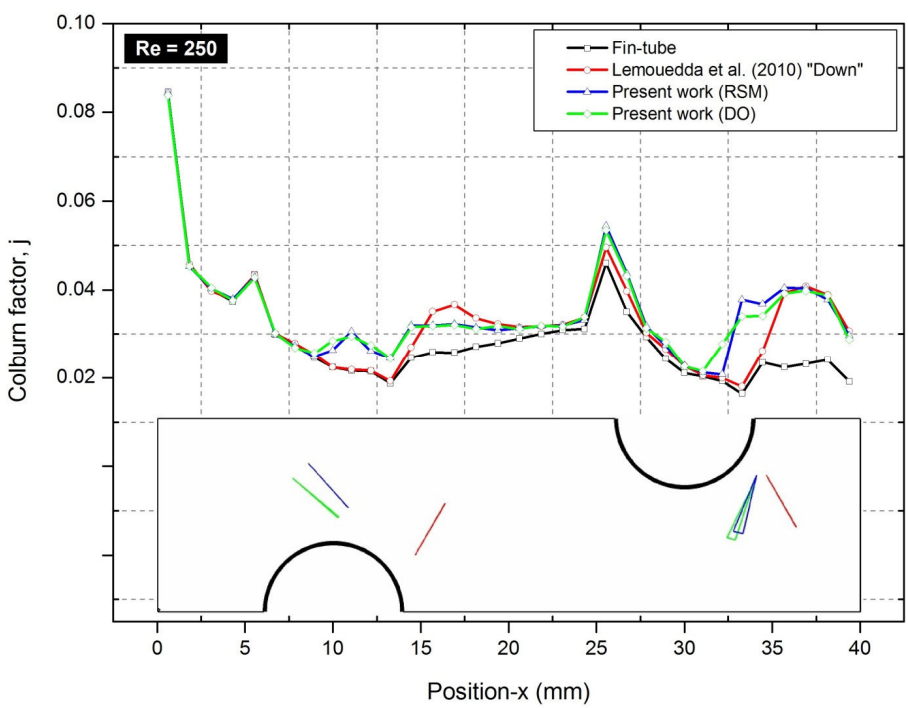

Figure 62 - Span-average Colburn factor (j) for the best results (Figure 57) for objective function j.

For both Fin-tube and enhanced configurations, the distributions of the span-average Colburn factor showed similar behavior up to the place of the leading edge of the vortex generator. The Colburn factor is very large at the inlet region where the temperature gradient is maximum at the leading edge of the fin bottom, and then both the velocity and thermal boundary layers develop along the flow direction that results in the decrease of the heat transfer. In front of the first and second tube, the span-average Colburn factor abruptly increases because of the formation of horseshoe vortices, which provide better mixing and enhances heat transfer in this 
region. The trends of the span-average Colburn factor for the enhanced configurations, beyond the location of the leading edge of the vortex generator, become quite different, as it can be seen to vortex generator placed in front of the first tube, Figure 60. The values of the span-average Colburn factor for the enhanced configuration were higher than fin-tube configuration.

Also for Figure 60, objective function JF, along the flow direction, for both RSM and DO approaches, the results were higher than survey literature work (Torii et al., 2002). The difference among the present works and Torii et al. (2002) are exactly after the VG close to the first and second tube. This difference is more pronounced in the second tube, close to wake region behind the tube, where the fluid recirculation with low velocity causes poor heat transfer; attack angle is fundamental to achieve better mixture between cold and hot fluid in this region.

Figure 61 presents the span-average Colburn factor for objective function $\mathrm{JF}^{\frac{1}{3}}$ with the same trends of the results for objective function JF, ie, there is significant augmentation heat transfer after the VG, which is more pronounced for the present work, especially for the DO approach in the second tube. Between RSM and DO approaches, the vortex generator position was decisive, since DO approach is disposed closer to wake region. However, RSM approach was slightly superior than DO approach close to $V_{G_{1}}$, because the $V G$ were placed further upstream adjacent to the tube in order to create a nozzle-like flow passage, which provide separation delay and narrows the wake. Between DO approach and Lemouedda et al. (2010), the positions are similar, however the difference of the attack and rolling angle are meaningful.

Figure 62 shows the span-average Colburn factor for objective function j. Over again, the results of the present work were superior than the result from survey literature (Lemouedda et al., 2010), especially after the VG. At the first tube, a nozzle-like flow passage between vortex generator (both RSM and DO approaches) and tube was observed, improving the heat transfer rate and delay the flow separation. This last statement is later evaluated. Also, the VG optimal position from optimization procedure was close to minimum flow area, where the average flow velocity is maximum. The maximum velocity causes the higher pressure drop behind the VG, which increases the swirl of the longitudinal vortices becoming it more persistent on 
downstream. At the second tube, the more pronounced enhancement heat transfer to the present work was due to VG is placed closer to tube, causing the fluid mixture in wake region (knowledge by lower heat transfer coefficient). Another important feature is the interaction between the vortices generated by VG, which is evaluated later through views applying the methodology called by Core Vortex.

A useful analysis is made considering the relation between enhanced and fin-tube configurations along the flow direction. This evaluation let us identify the region more enhanced, indicating where the visualization step should be focused. Figure 63 to Figure 65 present these results and indicate some trends already discussed in Figure 60 to Figure 62. For both objective functions, the relation of the Colburn factor was more enhanced after the VG, remarkable behind the second tube. In this wake region, the heat transfer coefficient is very low because the flow recirculation and low velocity. Thus, is fundamental to produce new vortical structure there to reach higher augmentation heat transfer. Moreover, the longitudinal vortex produced by the $\mathrm{VG}_{1}$ contributes for this, since the longitudinal vortices are very persistent along the flow direction. The influence of the VG was very notable, evaluated in Figure 63. VG configuration by the present work was placed in front of the first tube and the heat transfer was enhanced due to longitudinal vortex. VG arrangement by Torii et al. (2002) was placed close to rear part of the tube. Up to there, the heat transfer was not enhanced, keeping around 1.0. These features could also be observed in Figure 64 and Figure 65 to the first tube. Therefore, enhancement heat transfer was very pronounced behind the tube 2, for both objective functions, due to mixing flow in wake region. However, the longitudinal vortices along of the flow direction from $\mathrm{VG}_{1}$ were very important because the enhanced heat transfer occur along the entire fin length, causing an accumulative effect. 


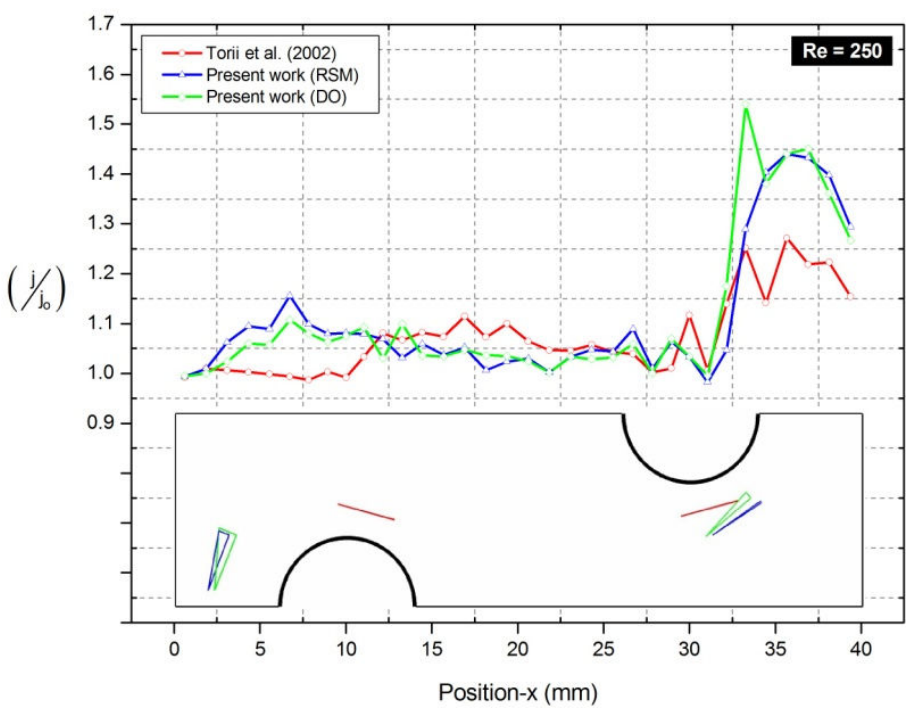

Figure 63 - Relation between span-average Colburn factor (j) with and without VG for the best results (Figure 55) for objective function JF.

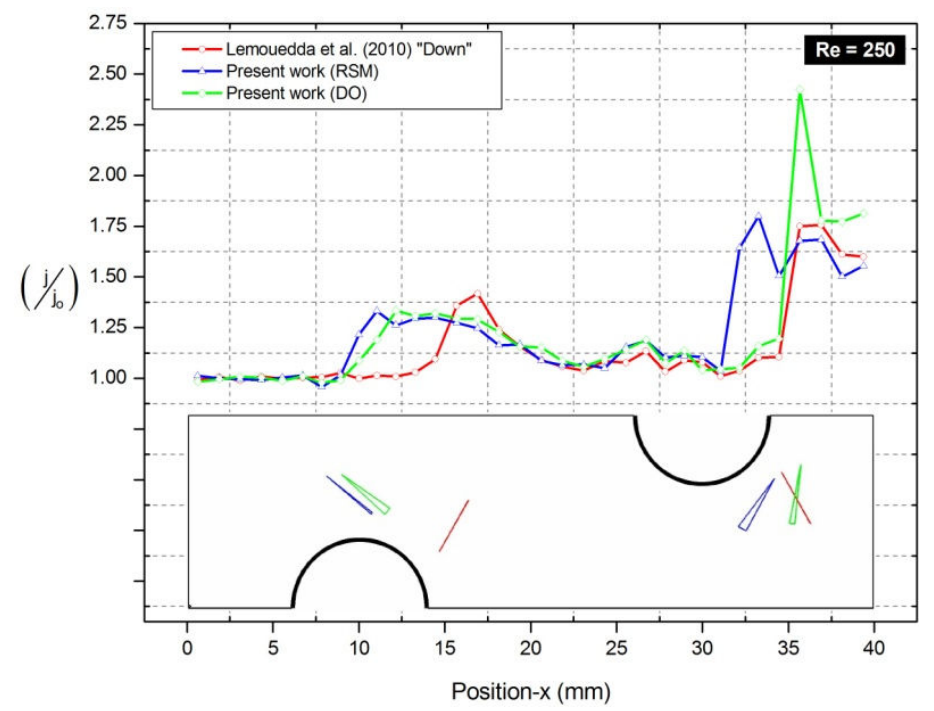

Figure 64 - Relation between span-average Colburn factor (j) with and without VG for the best results (Figure 56) for objective function $\mathrm{JF}^{\frac{1}{3}}$. 


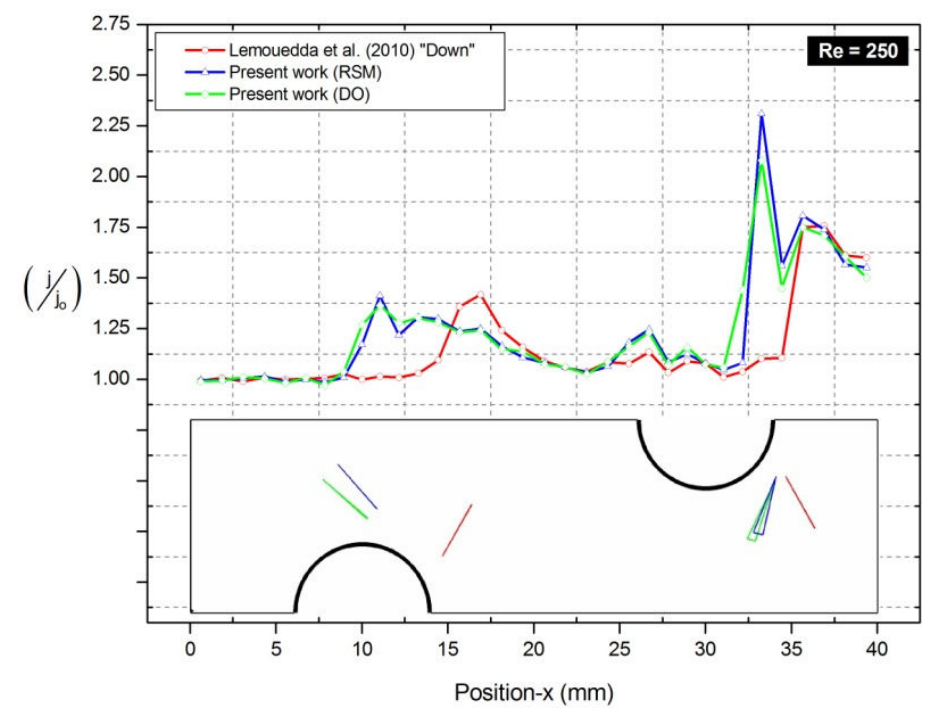

Figure 65 - Relation between span-average Colburn factor (j) with and without VG for the best results (Figure 57) for objective function $\mathrm{j}$.

Two methodologies, already implemented into post-processing software Ensight called by Vortex Core (together with streamlines) and Flow Separation, were used to clarify and explain some outlined results.

Vortex Core methodology helps visualizes the centers of swirling flow in a flow field. EnSight creates vortex core segments from the velocity gradient tensor of 3D flow field. Core segments can then be used as emitters for ribbon traces (streamlines) to help visualize the strength and nature of the vortices. Vortex Core is calculated according to algorithms based on techniques outlined by Sujudi and Haimes (1995) and Kenwright and Haimes (1997). This technique is linear and nodal, ie, it is based on decomposing finite elements into tetrahedrons and then solving closed-form equations to determine the velocity gradient tensor values at the nodes. Also, any variable with values at element centers are first averaged to element nodes before processing. The algorithm uses classification of vectors to determine whether the vortex core intersects any faces of the decomposed tetrahedron.

Separation Lines methodology (on 2D surfaces) helps visualize areas where flow abruptly leaves to the 2D surface in 3D flow fields. These lines are topologically significant curves on the 2D surface where flow converges and then separates (separation lines) from the surface into the $3 \mathrm{D}$ flow field. These line segments can be used as emitters for ribbon traces (streamlines) to help visualize flow interaction from 
the 2D surface into the 3D field, or displayed along with surface-restricted traces to help visualize the topology of the 2D surface. Ensight also creates separation lines from the velocity gradient tensor of the $3 \mathrm{D}$ flow field. It is calculated according to the phase plane algorithm presented by Kenwright (1998). This technique is linear and nodal, ie, 2D elements are decomposed into triangles, and then closed-form equations are solved to determine the velocity gradient tensor values at the nodes. Also, any variables with values at element centers are averaged to element nodes before processing.

In next analysis, each objective function was separately analyzed to allow identify the key flow features, since the optimization procedure found different VG arrangement. Additionally to outlined post-processing methodologies, is also presented the velocity plane at axis-z to evidence the results discussed from Figure 63 to Figure 65. The planes positions at axis-z were defined to match the horseshoe vortexes generated in front of the tubes. This assessment helps to view the interaction among tubes and VG and these vortices.

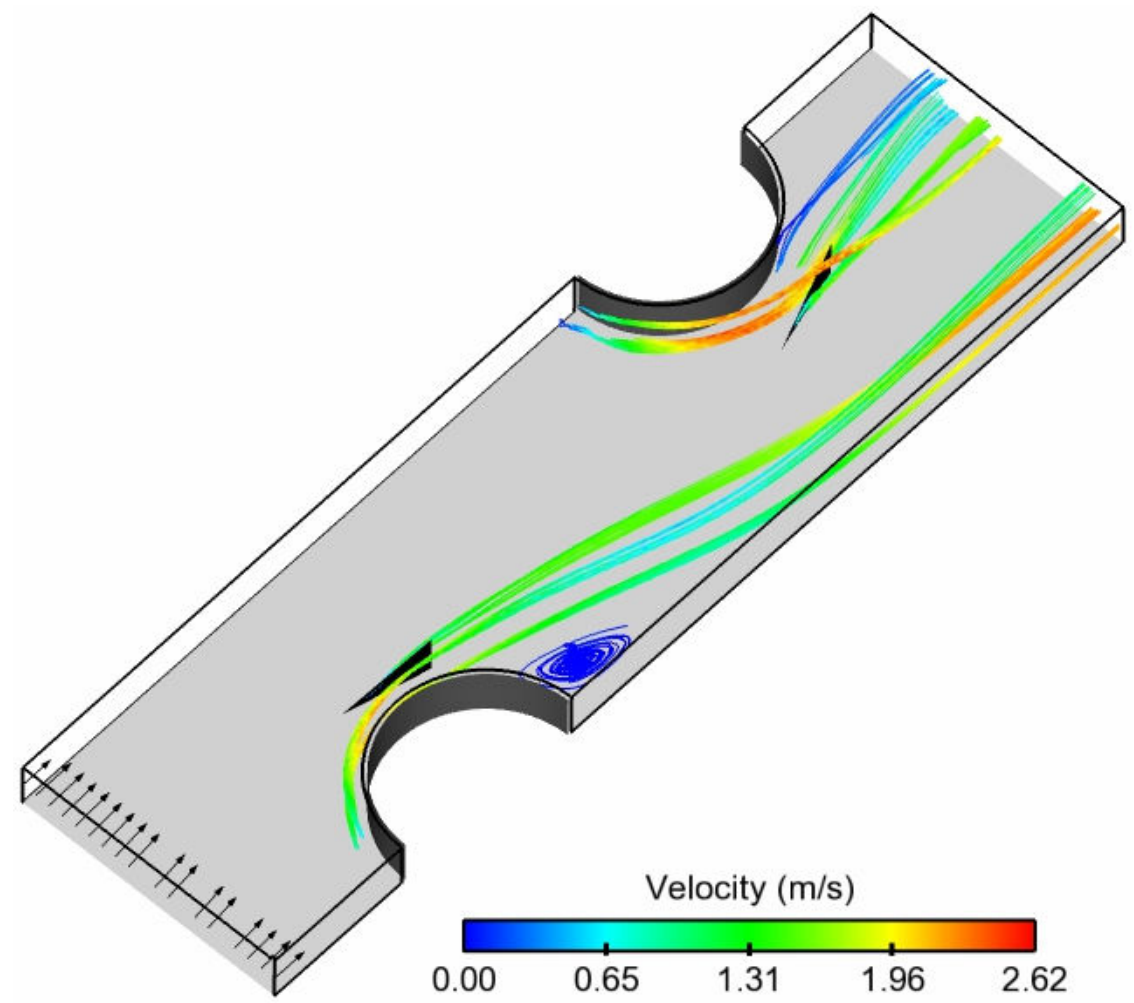

Figure 66 - Streamlines emitted from Core vortex by Torii et al. (2002) arrangement. 


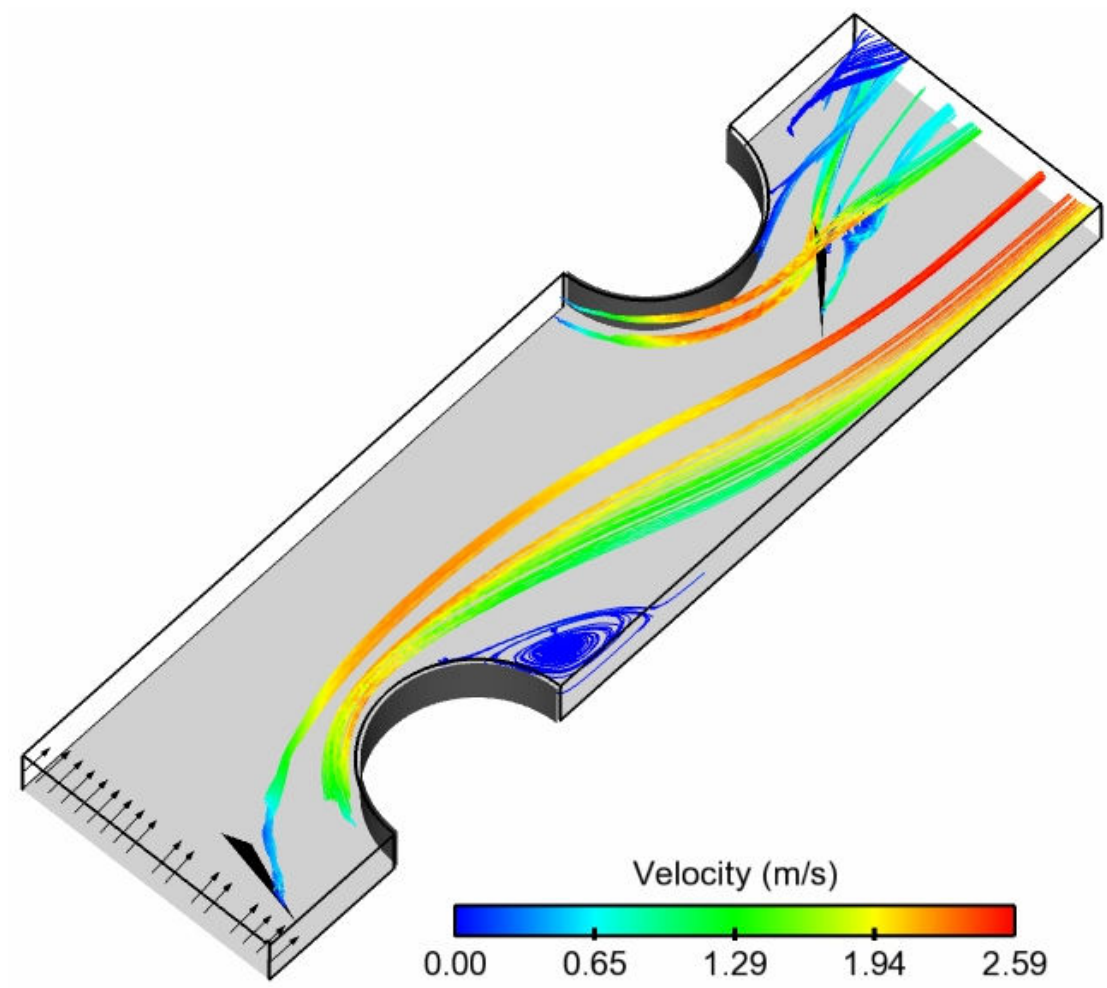

Figure 67 - Objective function JF. Streamlines emitted from Core vortex by present work (DO).

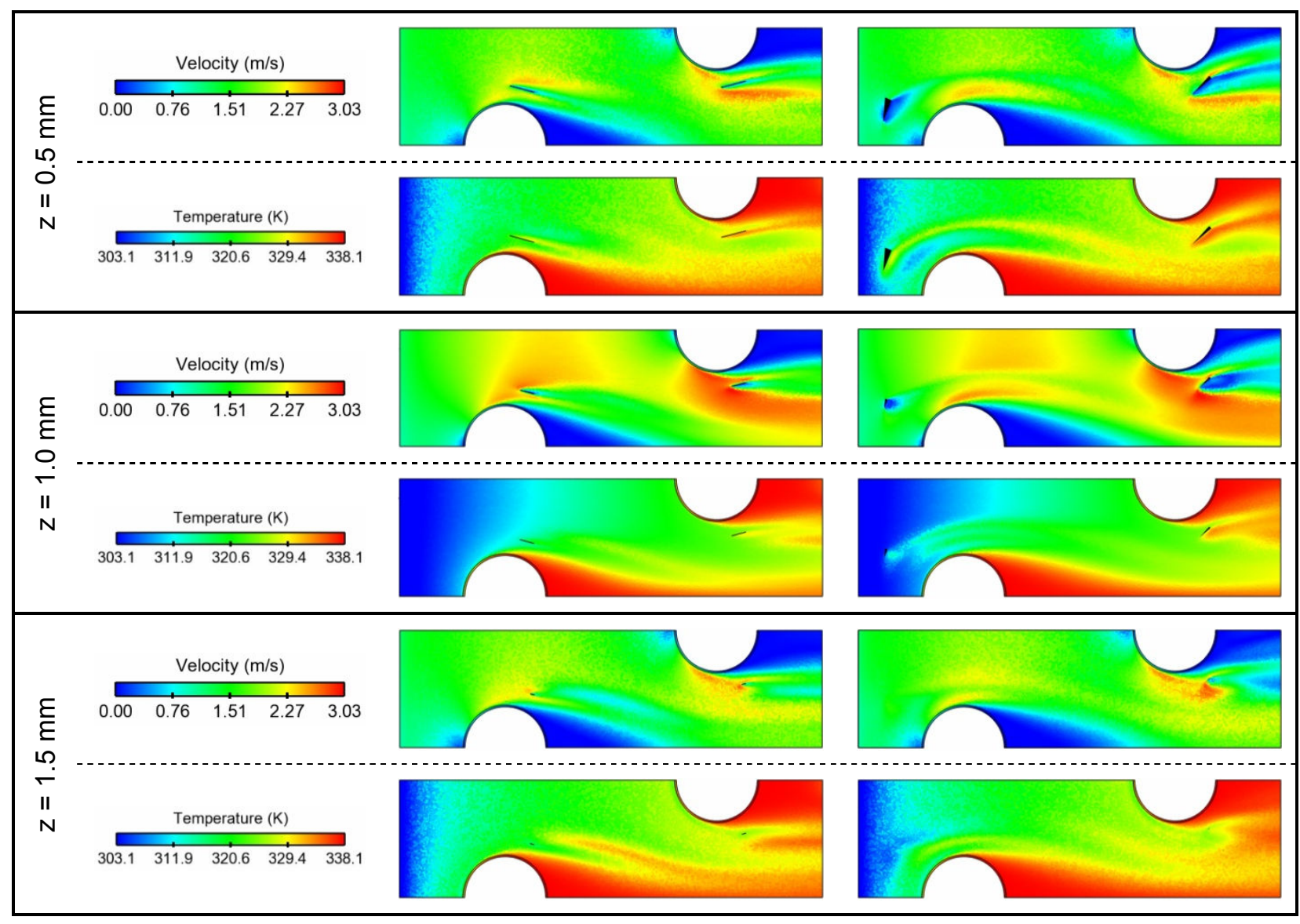

(a) Torii et al. (2002).

(b) JF present work (DO).

Figure 68 - Velocity and temperature field planes at axis-z, according to Figure 55. 


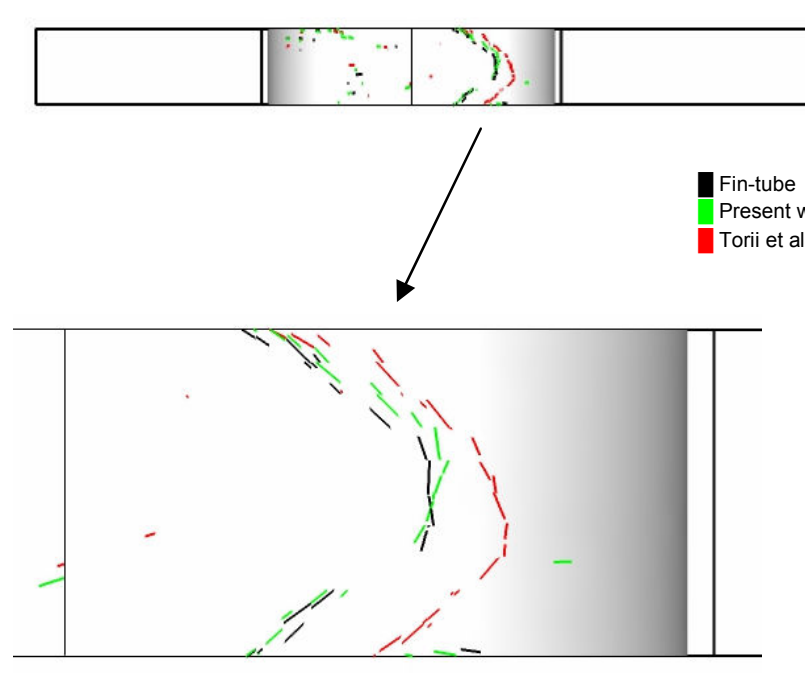

(a)

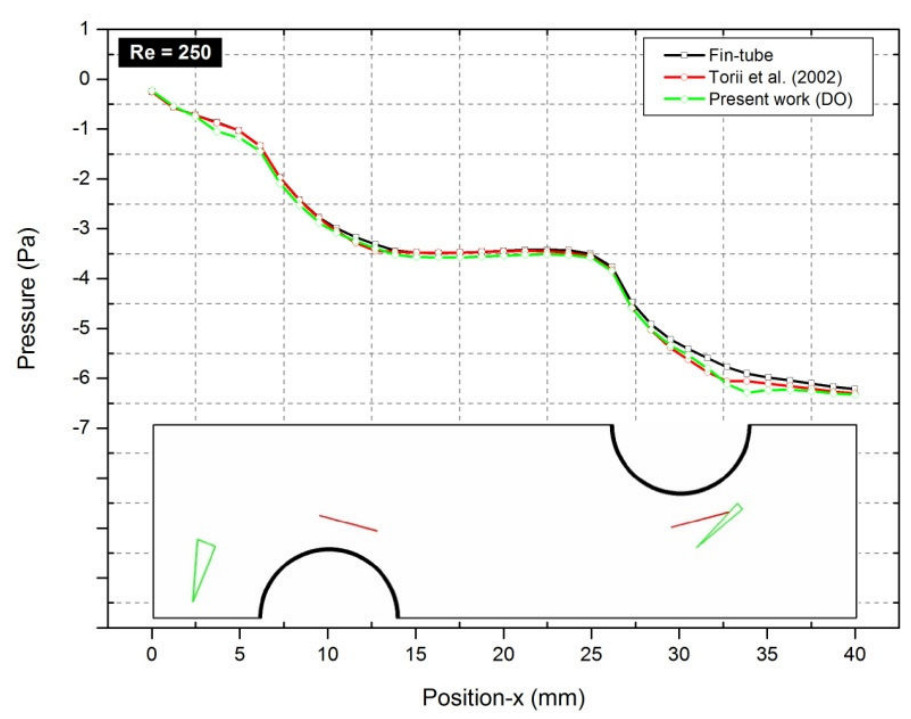

Figure 70 - Pressure profile considering the objective function JF.

Figure 66 to Figure 70 are related to objective function JF, according to Figure 63 . These set of analysis were jointly necessary to understanding the thermal-hydraulic performance of the VG arrangement along of the flow direction. Figure 66 and Figure 67 show the streamlines emitted from core vortex considering the configuration by Torii et al. (2002) and by present work (DO), respectively. Thermal analysis assessment was already outlined, Figure 60 and Figure 63, and the discussion presented herein especially focused on the key features to hydraulic analysis. However, in augmentation heat transfer studies is evident the narrow thermal- 
hydraulic correlation. Both configurations were able to produce secondary flow, Figure 66 and Figure 67, which increased the heat transfer coefficient. For both, the main vortex produced by $V G_{1}$ was not directed onto $V G_{2}$, although the vortices were persistent along of the flow direction; this contributes to decrease the global pressure drop. For the present work (DO), $V_{G_{1}}$ arrangement does not cooperate to decrease the pressure drop, especially due to soft separation delay on the first tube, differently as for Torii et al. (2002) configuration. These assessments are evidenced in Figure 69 and Figure 70 . In this case, the $\mathrm{VG}_{1}$, by present work (DO), mainly increased the heat transfer rate and, by Figure 70, the pressure drop, faced to fin-tube pressure profile, was also increased. On the other hand, Torii et al. (2002) slightly decreased the pressure drop, which was evident the separation delay, Figure 69. These could already be concluded considering that the $\mathrm{VG}_{1}$ position by present work (DO) is not close to the first tube. However, for the second tube, the separation delay is more pronounced in the present work (DO) than in the Torii et al. (2002), decreasing the global pressure drop. In fact, considering fin-tube, flow separation delay was due to the flow acceleration through nozzle between VG and tube, shown in Figure 70; this assessment was already discussed by Torii et al. (2002). Flow acceleration is evidenced in Figure 68. Moreover, the flow directed toward the wake region increased the heat transfer, explaining the results showed in Figure 60 and Figure 63, which conducted to higher fluid mixing and consequently a stronger heat transfer between the fluid and the plate-fin wall, Figure 68. Also, evaluating the results in Figure 67, the difference between attack angle and rolling angle for $V G_{2}$ are evaluated. Attack angle contributes to accelerate the flow behind the tube, whereas the rolling angle was important to avoid the direct collision between horseshoe and VG, which could increase the global pressure drop. Analyzing the flow pattern produced by the present work (DO) arrangement, the main vortex generated by $\mathrm{VG}_{1}$ and $V G_{2}$ were co-clockwise while for Torii et al. (2002) was counter-clockwise for $V G_{1}$ and co-clockwise for $V G_{2}$. These different patterns are justified by symmetry of the VGs for Torii et al. (2002) and the lack thereof for the present work (DO).Therefore, clockwise seems to be a secondary subject compared to relative position between VGs and tubes. Figure 71 to Figure 75 are related to objective function $\mathrm{JF}^{\frac{1}{3}}$ according to Figure 64 . 


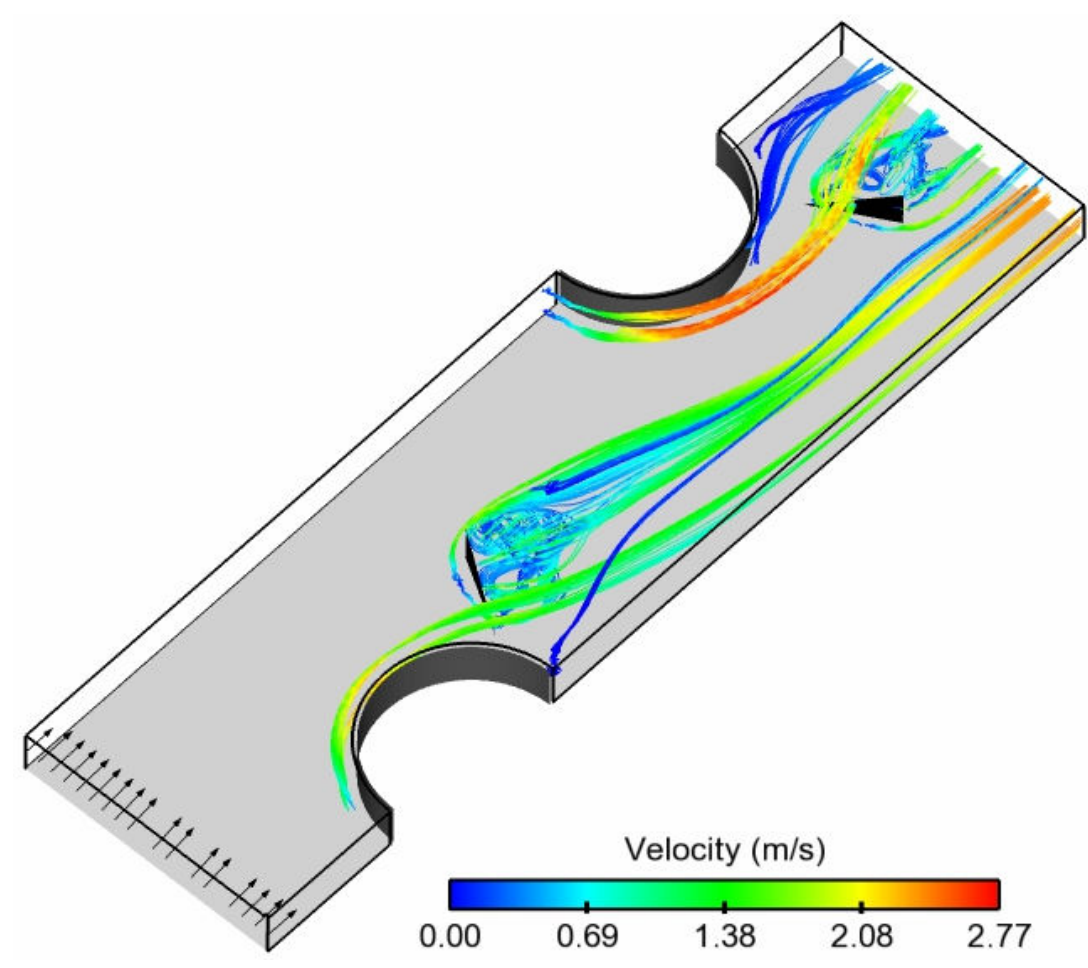

Figure 71 - Streamlines emitted from Core vortex by Lemouedda et al. (2010) arrangement.

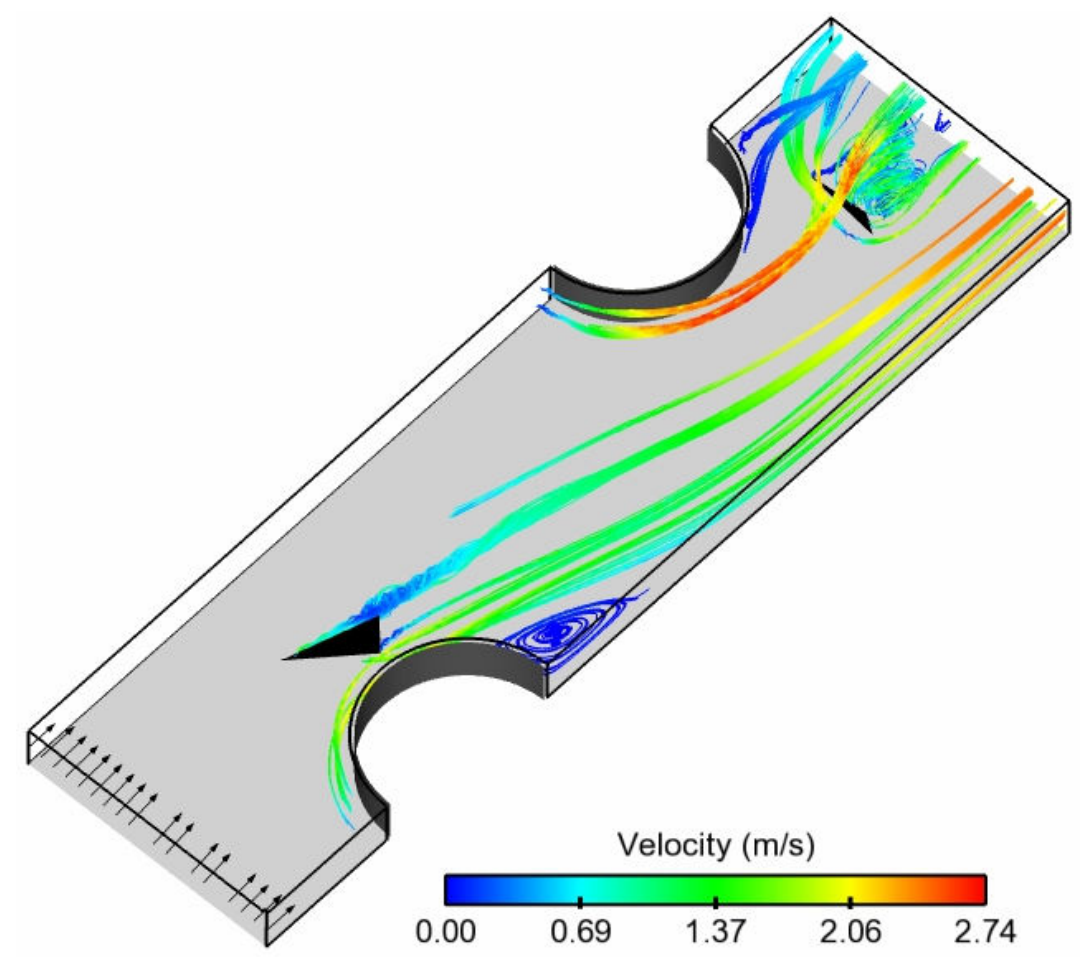

Figure 72 - Objective function $\mathrm{JF}^{\frac{1}{3}}$. Streamlines emitted from Core vortex by present work (DO). 


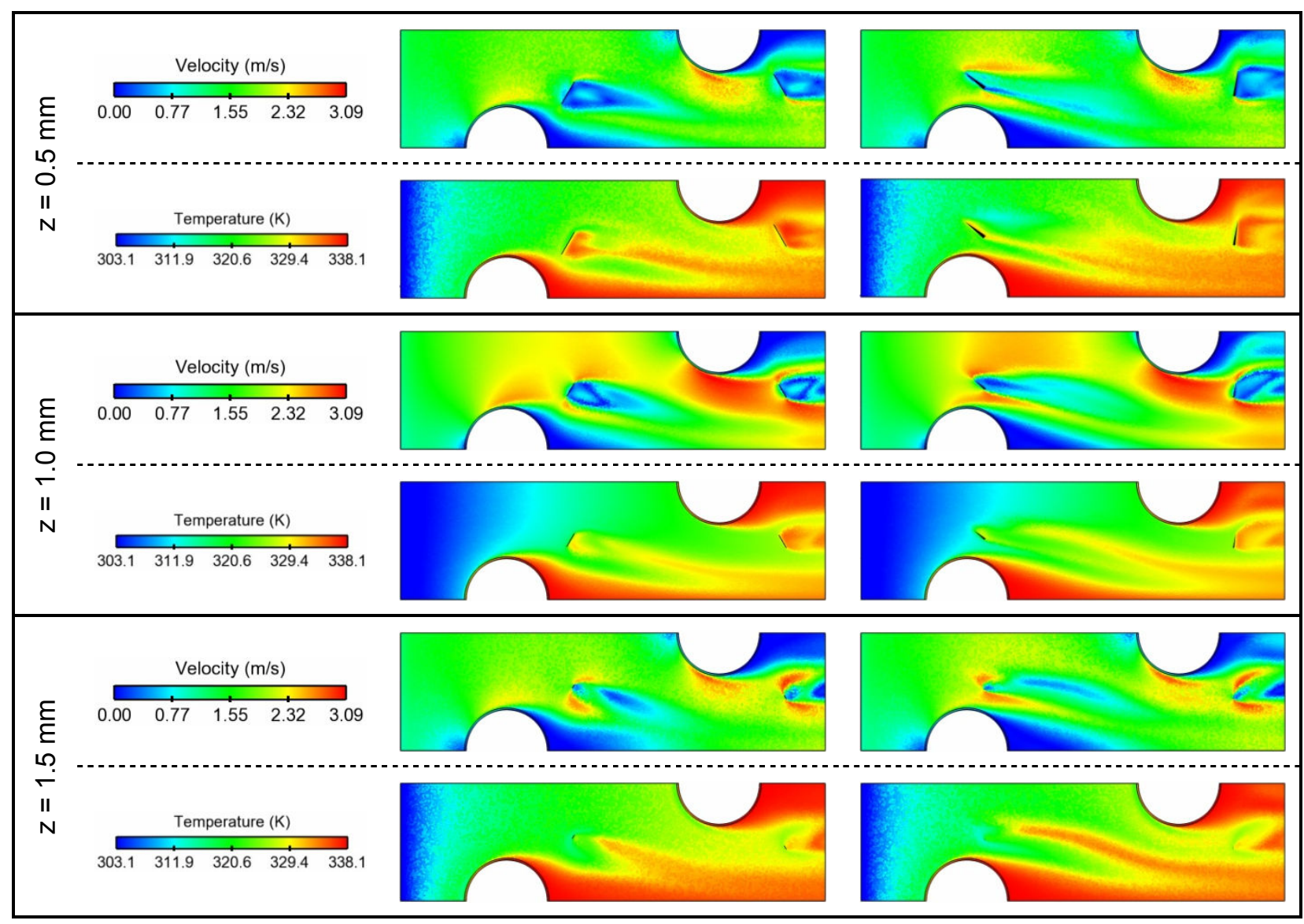

(a) Lemouedda et al. (2010).

(b) $\mathrm{JF}^{\frac{1}{3}}$ present work (DO).

Figure 73 - Velocity and temperature field planes at axis-z, according to Figure 56.

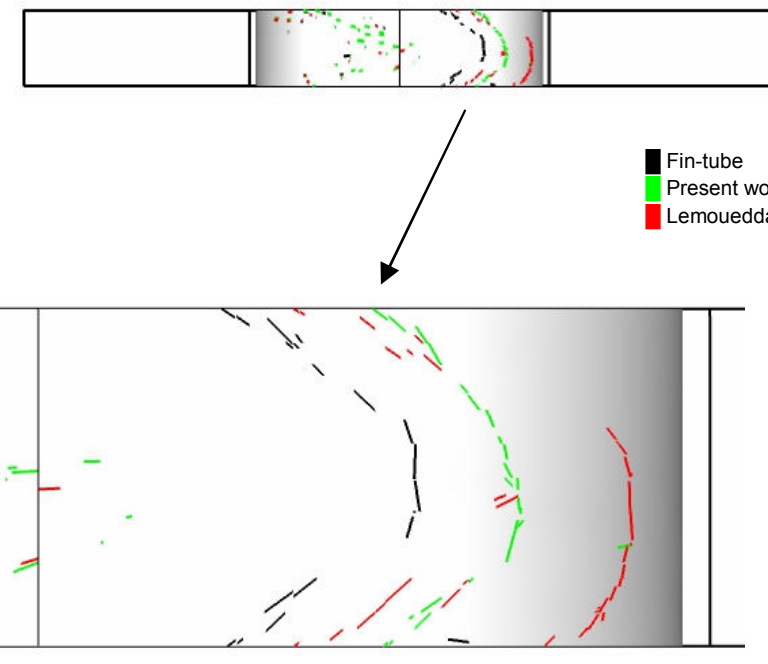

(a)

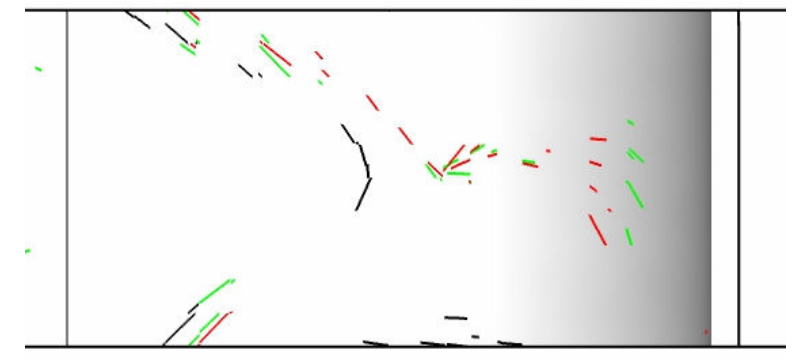

(b)

Figure 74 - Objective function $\mathrm{JF}^{\frac{1}{3}}$. Flow separation profile on (a) tube 1, (b) tube 2. 


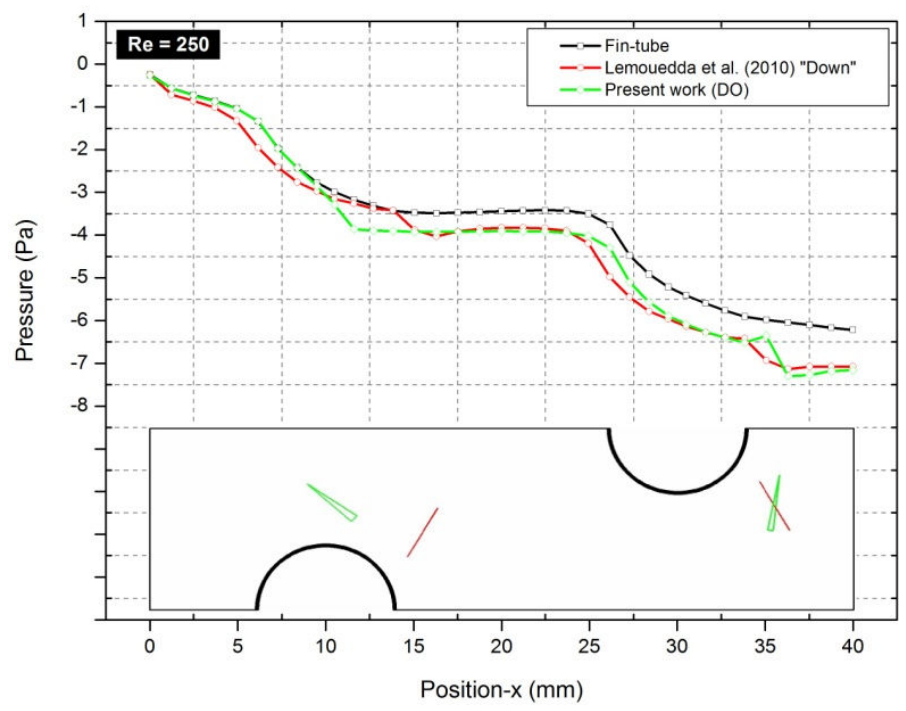

Figure 75 - Pressure profile considering the objective function $\mathrm{JF}^{\frac{1}{3}}$.

Figure 71 and Figure 72 show the streamlines emitted from core vortex considering the configuration by Lemouedda et al. (2010) and by the present work (DO), respectively. Again, thermal analysis assessment was already outlined, Figure 61 and Figure 64, and the discussion presented herein focused especially on the key features to hydraulic analysis. Reminding, objective function $\mathrm{JF}^{\frac{1}{3}}$ differently weighted the thermal-hydraulic performance of the heat exchanger, faced to objective function JF. In fact, the optimal configuration found by optimization procedure was different for each objective function, although some flow patterns were slightly similar, according to discussion below. VG arrangements for Lemouedda et al. (2010) and by present work (DO) were able to produce secondary flow, Figure 71 and Figure 72, increasing the heat transfer coefficient. For both, the longitudinal main vortex produced by $\mathrm{VG}_{1}$ was not directed onto $\mathrm{VG}_{2}$, which could contribute to decrease the global pressure drop. Separation delay on tube 1 was significant for the present work (DO), whereas for the Lemouedda et al. (2010), Figure 74, were identified two separation lines; the first was very soft whereas the second was bruising. These profiles do not make sense considering the common-flow-down configuration and, in this case, the high value of the attack angle could justify this profile, beyond the flow recirculation behind the tube. However, the pressure drop in this region is another important behavior, Figure 75. On the second tube, separation lines profile was not clearly defined because the strong flow mixture behind the tube. These are evidenced by analyzing 
Figure 73. In general, Lemouedda et al. (2010) and the present work (DO) presented similar pressures drop, $14.3 \%$ and $15.8 \%$, respectively. However, the enhancement heat transfer changed a lot, $11.6 \%$ and $17.6 \%$, respectively. In Figure 73 , the air flow directed toward the wake region increased the heat transfer, explaining the results showed in Figure 61 and Figure 64. In the same way as for objective function JF, the $V_{G_{2}}$ attack and rolling angles presented the same behavior to the objective function $\mathrm{JF}^{\frac{1}{3}}$, ie, attack angle contributed to flow acceleration behind the tube and rolling angle was important to avoid the direct collision between horseshoes and VG. Main vortex rotation produced by $\mathrm{VG}_{1}$, according to Lemouedda et al. (2010) arrangement, was co-clockwise and counter-clockwise for $V G_{2}$. For the present work (DO), $V_{1}$ generated vortices in counter-clockwise; however, for $V G_{2}$ the vortices rotation is not promptly defined because the higher attack angle. In fact, in Figure 72, the vortices by $V G_{2}$ were like a transverse vortex since the attack angle $\left(\theta_{2}\right)$ was around the $-90^{\circ}$ (or $270^{\circ}$ according to reference in present work).

Figure 76 to Figure 79 are related to objective function j, according to Figure 65.

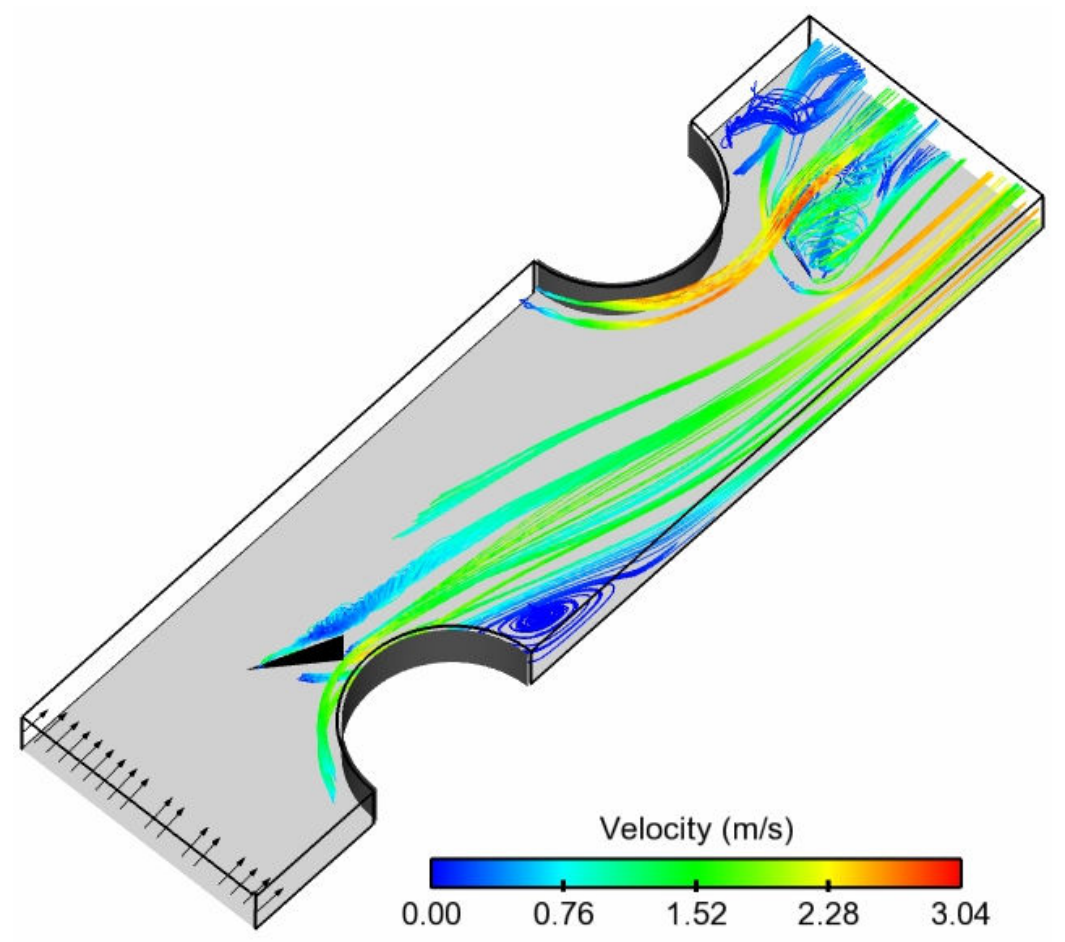

Figure 76 - Objective function j. Streamlines emitted from Core vortex by present work (DO). 


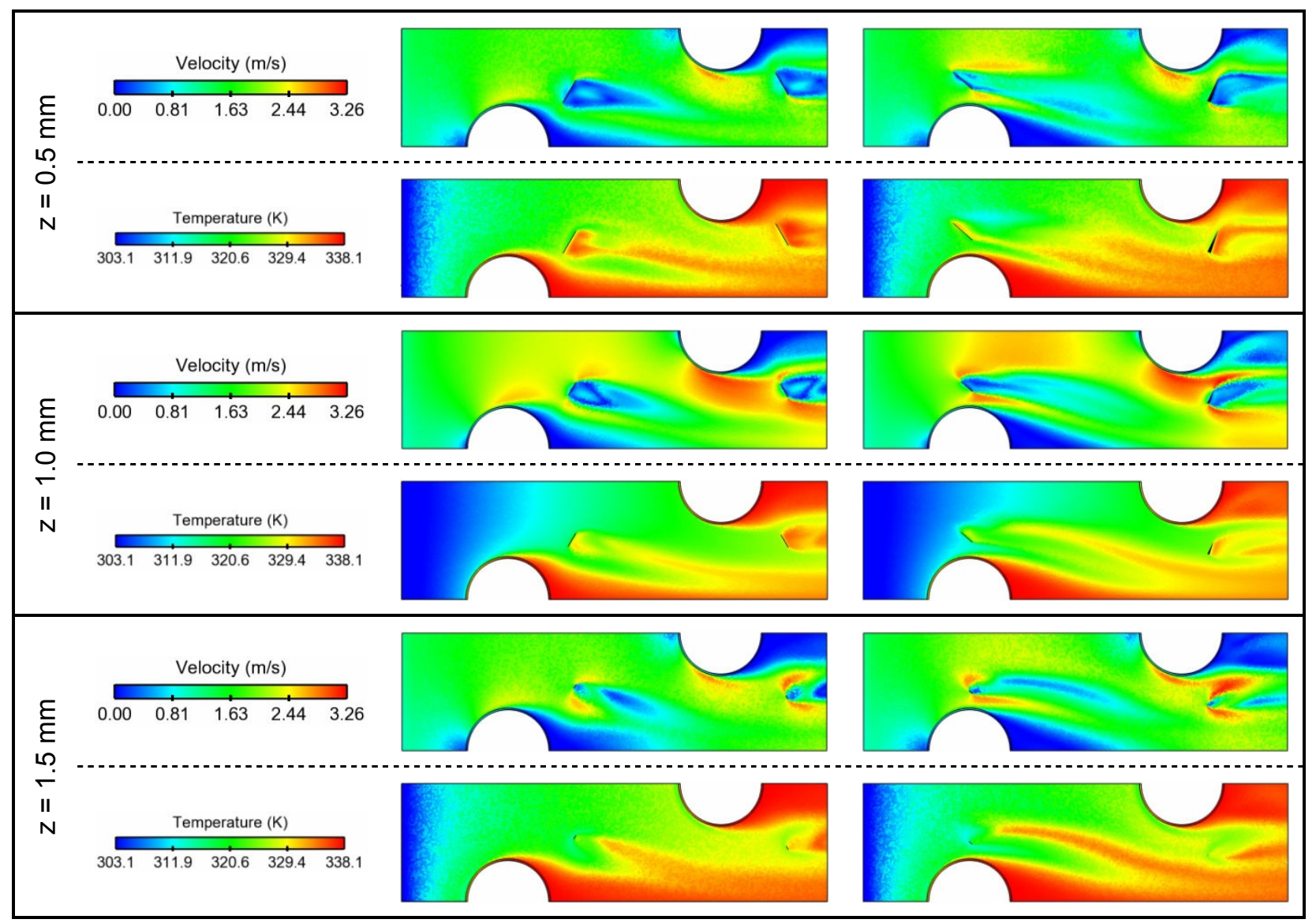

(a) Lemouedda et al. (2010).

(b) j present work (DO).

Figure 77 - Velocity and temperature field planes at axis-z, according to Figure 57.

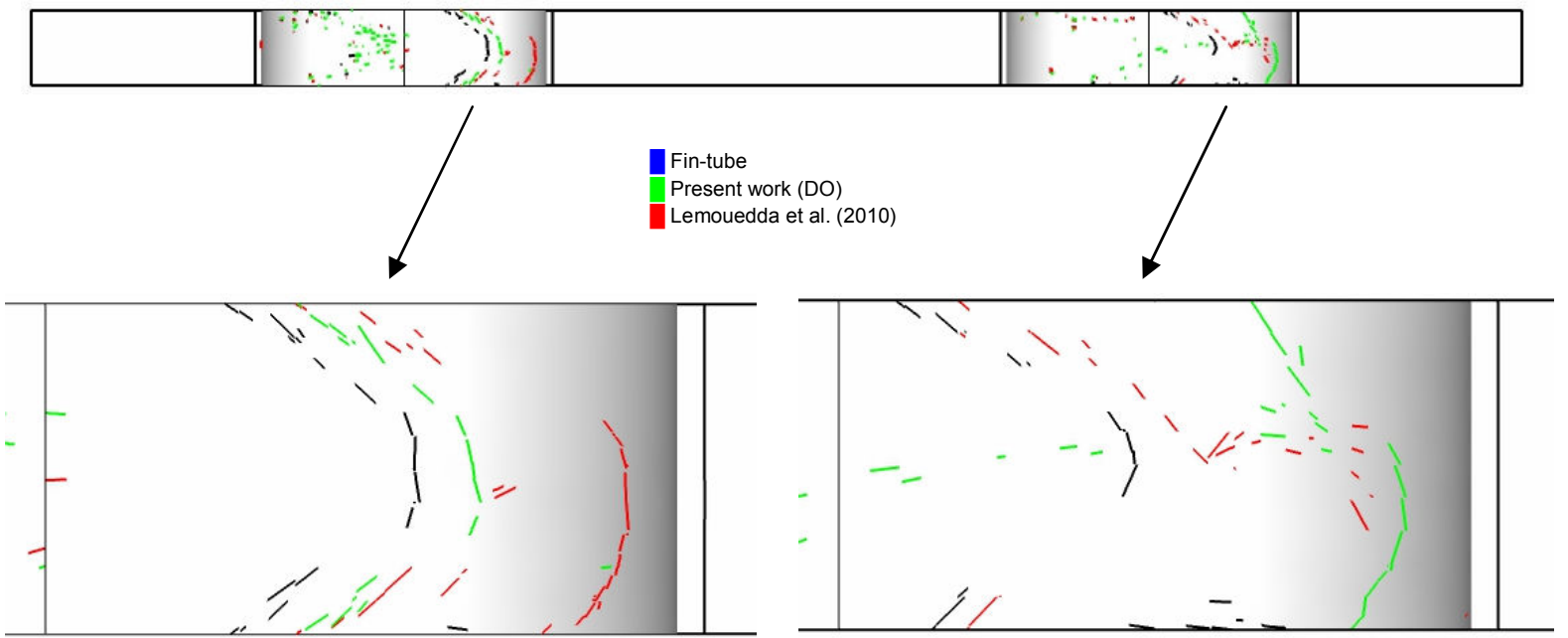

(a)

(b)

Figure 78 - Objective function j. Flow separation profile on (a) tube 1, (b) tube 2. 


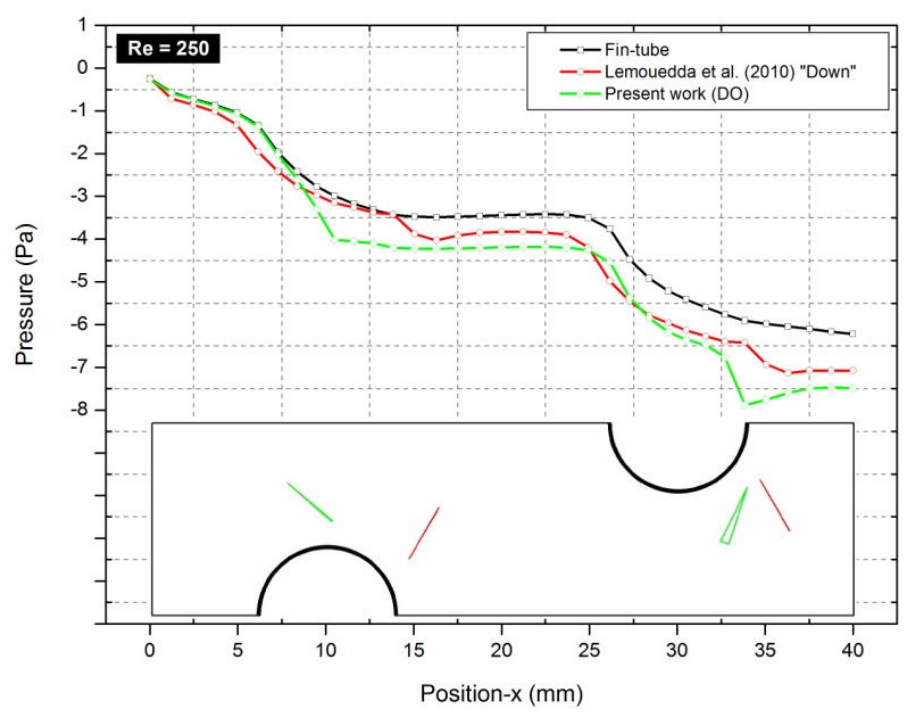

Figure 79 - Pressure profile considering the objective function $\mathrm{j}$.

Opposite to objective functions $\mathrm{JF}$ and $\mathrm{JF}^{\frac{1}{3}}$, optimization procedure to objective function $\mathrm{j}$ was conducted as a simple-objective, ie, the friction factor was only a consequence and is not "controlled". However, an interesting result facing the configuration found to $\mathrm{j}$ and $\mathrm{JF}^{\frac{1}{3}}$, according to Table 8 for the present work (DO), showed that these configurations are very similar. In fact, for $\mathrm{JF}^{\frac{1}{3}}$ the global result was $12 \%$, whereas for objective function $\mathrm{j}$ the relation $\mathrm{JF}^{\frac{1}{3} *}$ was $11 \%$. For the first tube, the VG were almost placed in the same position, changing a lot for rolling angle. In fact, this observation showed that the rolling angle was really important to minimize the pressure drop. Exploiting this a bit further, the projected frontal area of the $\mathrm{VG}_{1}$ to $\varphi=+3^{\circ}$ is higher than $\varphi=-16^{\circ}$. For the second tube, the velocity field slightly changed and, as was expected, the maximum velocity to objective function $\mathrm{j}$ was higher than $\mathrm{JF}^{\frac{1}{3}}$, where the flow acceleration is associated to higher pressure drop, Figure 77. Flow pattern from Lemouedda et al. (2010) configuration, was already discussed and is not brought in again. For objective function $\mathrm{j}$, the flow pattern and the key features were very similar to $\mathrm{JF}^{\frac{1}{3}}$, Figure 72 and Figure 76 . Thus, the discussion is conducted, for the present work (DO), to compare some interesting result among objective functions $\mathrm{JF}, \mathrm{JF}^{\frac{1}{3}}$ and $\mathrm{j}$. The concern about pressure drop is differently weighted for each objective function, ie, pressure drop is more significant for $\mathrm{JF}$ than $\mathrm{JF}^{\frac{1}{3}}$. Moreover, pressure drop is only a consequence for objective 
function $\mathrm{j}$. Therefore, rolling angle of the first VG showed different value for each objective function: $-40^{\circ}$ (for $\mathrm{JF}$ ), $-16^{\circ}$ (for $\mathrm{JF}^{\frac{1}{3}}$ ) and $+3^{\circ}$ (for j), according to Table 8 . Therefore, the rolling angle was more inclined when pressure drop is a great concern and turn to current position when heat transfer rate is the main goal. This same behavior was not observed for the second tube and the relation between rolling angle and pressure drop is not conclusive. Thus, these conclusions about the pressure drop corroborates with results presented in Figure 70, Figure 75 and Figure 79. Evaluating the final pressure in the outlet-domain, the local pressure difference between fin-tube and present work (DO) arrangement was higher to objective function $\mathrm{j}$ than $\mathrm{JF}$ and $\mathrm{JF}^{\frac{1}{3}}$. Also, comparing the position found by optimization procedure, for each objective functions (JF, $\mathrm{JF}^{\frac{1}{3}}$ and $\mathrm{j}$ ) an important question is raised to understand the root cause for $V_{G_{1}}$ is placed in front of the tube for JF and close to minimum flow area for $\mathrm{JF}^{\frac{1}{3}}$ and $\mathrm{j}$. The strength of the longitudinal vortex is defined by difference between the pressure flow and pressure behind the VG. Thus, the higher pressure difference means that more persistent is the longitudinal vortex along of the fin. However, the local pressure loss associated with this flow acceleration is also evident. In fact, when VG is placed close to minimum flow area, it is expected higher global pressure drop and, consequently, higher heat transfer. Therefore, this assessment could explain the position of the VG for objective function $\mathrm{JF}$, taking into account that the pressure drop is more relevant to $\mathrm{JF}$ than $\mathrm{JF}^{\frac{1}{3}}$ and $\mathrm{j}$.

Perhaps, it could be claimed that the first vortex generator is very important to enhancement heat transfer along of the fin, with penalty in the pressure drop. If separation delay is observed in the first tube, the global impact of the $V G_{1}$ on flow could be mitigated. However, when the objective function is set to minimize the friction factor, it is expected that the $V G_{1}$ configuration found by optimizer could be neutral, eg, either $\theta=0^{\circ}$ or immersed into the wake region.

Figure 80 to Figure 82 are related to objective function f, according to Figure 58. 


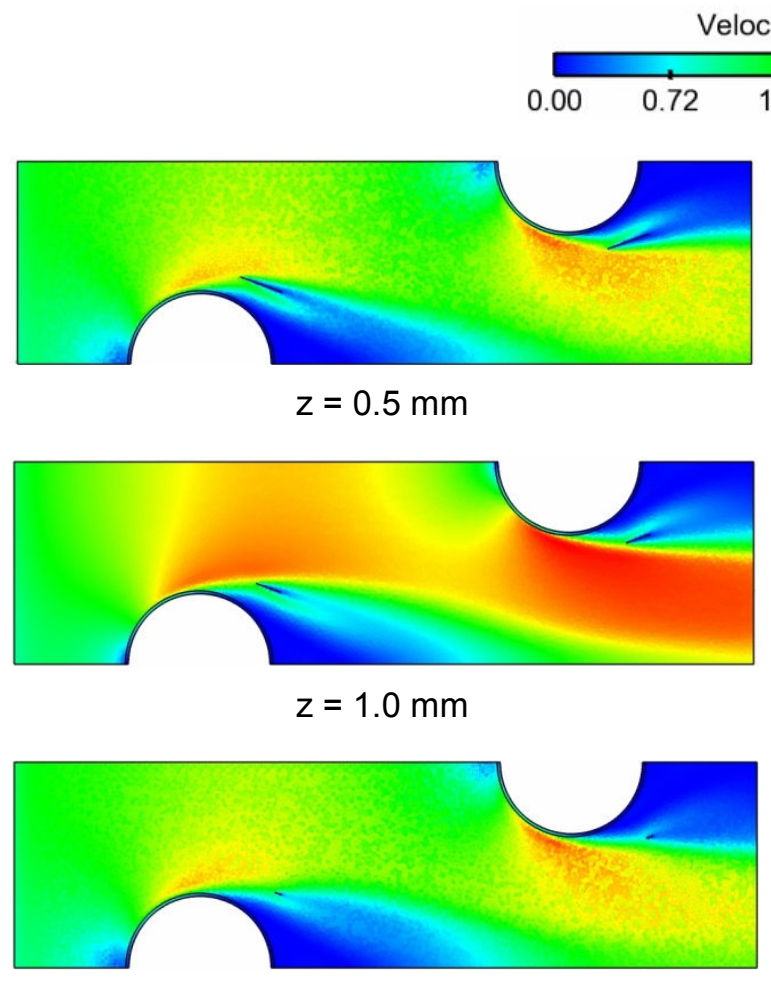

$z=1.5 \mathrm{~mm}$

(a) Lei et al. (2010)

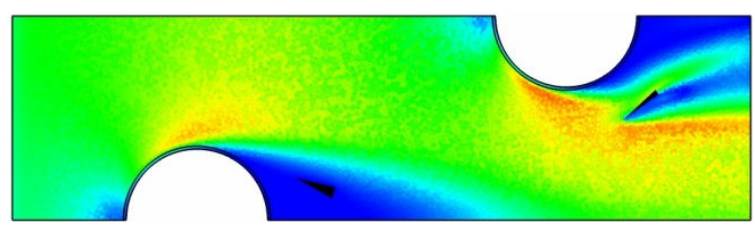

$z=0.5 \mathrm{~mm}$

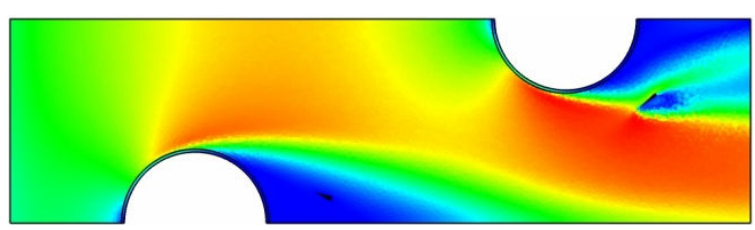

$\mathrm{z}=1.0 \mathrm{~mm}$

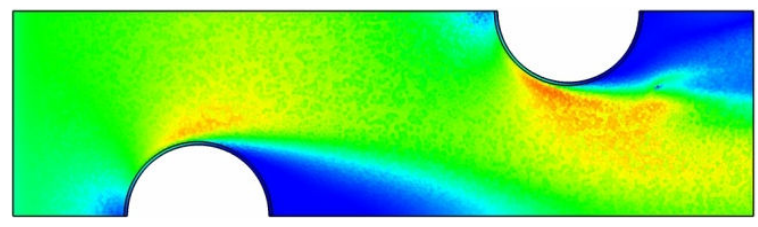

$\mathrm{z}=1.5 \mathrm{~mm}$

(b) Present work (DO)

Figure 80 - Objective function $\mathrm{f}$. Velocity planes at axis-z.

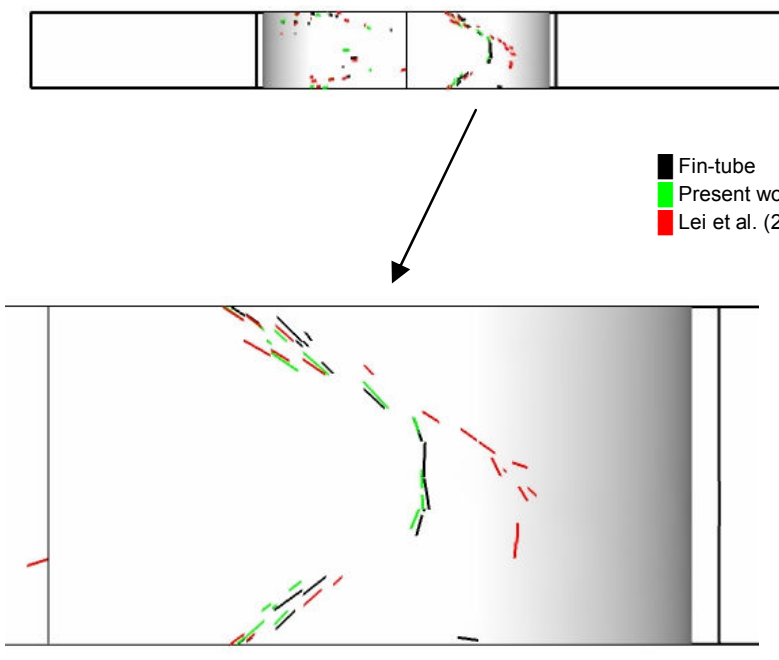

(a)

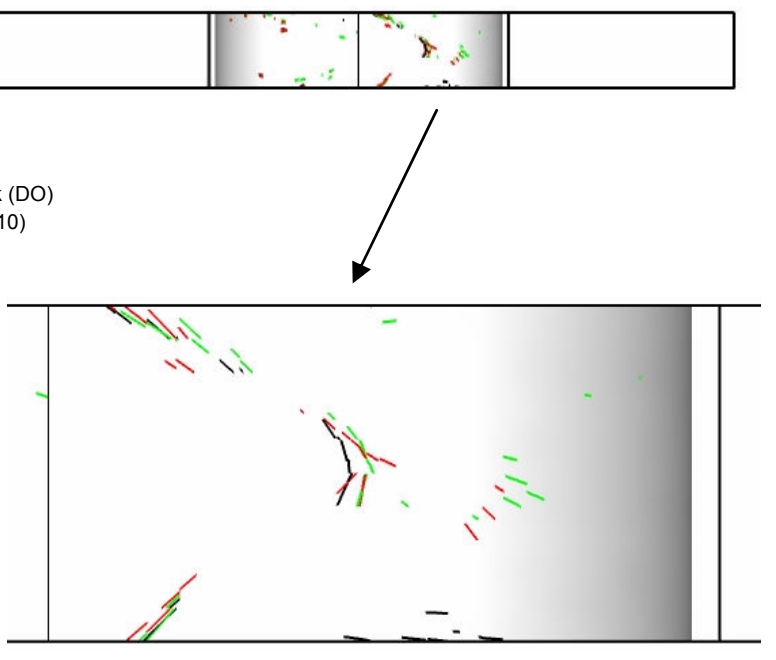

(b)

Figure 81 - Objective function f. Flow separation profile on (a) tube 1, (b) tube 2. 


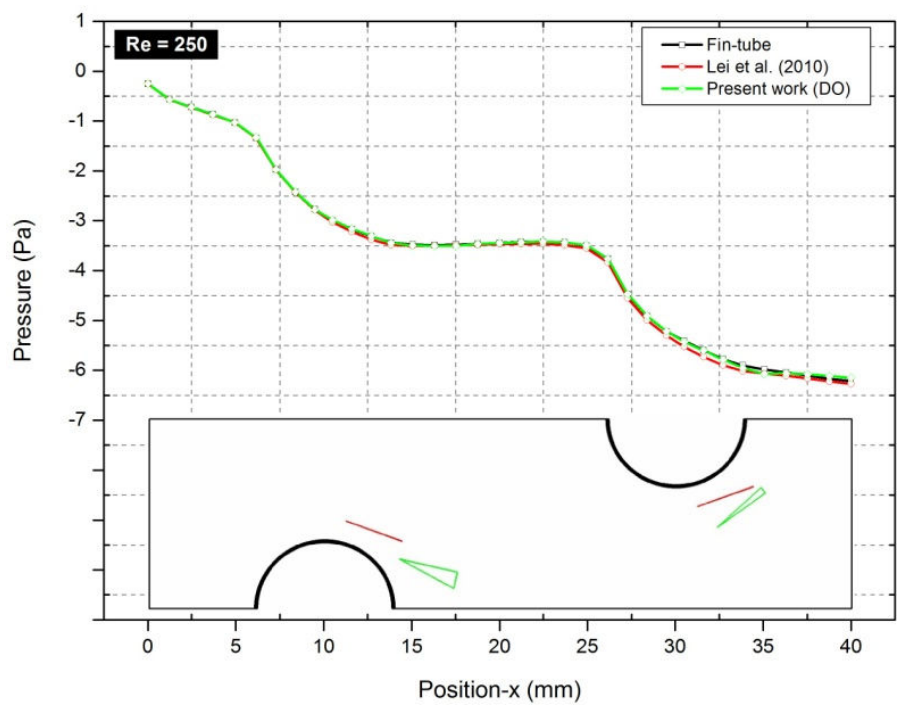

Figure 82 - Pressure profile considering the objective function $\mathrm{f}$.

Figure 80 shows the pressure field at axis-z for the present work (DO) and the best literature research, according to Figure 58. Pressure field by Lei et al. (2010) arrangement was symmetric and showed the called nozzle between VG and tube, causing the flow acceleration, as it was already discussed. This approach proposed by Torii et al. (2002), known as common-flow-up configuration, was widely cited during the above text. This approach is really interesting because the VG position could decrease the flow separation delay on tubes, beyond increase the heat transfer rate. However, there is a net effect of the vortex generator when VG is inserted into the flow, ie, the VG naturally increase the pressure drop, where the flow separation delay could decrease the global penalty. For the first tube, Figure 81 showed that the separation delay was achieved for configuration by Lei et al. (2010) and none to configuration by present work (DO). For the second tube, separation delay was very soft for both arrangements. Result by present work (DO) was already expected considering that the VG position, Figure $80 \mathrm{~b}$, was placed behind the first tube. Figure 82 showed that the pressure profile from Lei et al. (2010), along the fin, was lower than present work (DO) and also to fin-tube in some regions. The configuration found by optimization procedure (DO) suggests that the $V G_{1}$ does not contribute to minimize the objective function $\mathrm{f}$, Figure $80 \mathrm{~b}$. Therefore, an additional analysis was conducted, which the $V \mathrm{VG}_{1}$ was removed and $V \mathrm{G}_{2}$ was kept in. The results indicated a difference of $0.004 \%$, ie, the results were numerically equal. Thus, considering the objective function $\mathrm{f}$, the $\mathrm{VG}_{1}$ was irrelevant. Also, considering the present work (DO) 
arrangement, another analysis was made positioning the $V G_{1}$ symmetrical to $V G_{2}$. The rationale for this analysis was to evaluate the local effect on the second tube, Figure 82, on the first tube. The result was $4 \%$ higher than previous optimal configuration. These corroborate with the last assessment which stating that the $\mathrm{VG}_{1}$ was important to enhancement heat transfer and, consequently, increased the global pressure drop. However, for $V G_{2}$ it was observed the augmentation of the heat transfer, beyond decreased pressure drop due to separation delay on the second tube.

\subsubsection{Flow pattern for $\operatorname{Re}=1400$}

The analysis conducted for $\operatorname{Re}=1400$ is similar to presented for $\operatorname{Re}=250$ and, as much as possible, a comparison was made between them.

Global results for each objective functions evaluated herein were faced to researches mentioned on the Table 8, Figure 83 to Figure 86. The results for both approaches adopted in this work (RSM and DO) were superior than reported by works in open literature for all objective functions analyzed. Also, global results from DO approach were significantly higher than RSM approach, more pronounced for $\operatorname{Re}=1400$ than $\mathrm{Re}=250 . \mathrm{RSM}$ quality and initial population applied on Genetic Algorithm could contribute to this difference between DO and RSM. Another aspect, not easy to investigate, is about the local optimal density on solution space for each Reynolds number. Solution observed for $\operatorname{Re}=250$ seems to be smoother than $\operatorname{Re}=1400$ and therefore, the optimizer could find the local optimal easier on dense solution space. Thus, initial population, mutation and crossover parameter applied in the Genetic Algorithm could explain this difference between DO and RSM results. This investigation is not the focus of this work and this is suggested as a future work.

For the objective function JF, Figure 83, among the configuration considered by literature works, Torii et al. (2002) presented the better results; however, the results for both DO and RSM approaches were much higher than the results reported by Torii et al. (2002). In Figure 87a, the position and angle among the present work and the literature arrangements were very different for both tubes. Moreover, the optimal 
configuration found for $\mathrm{Re}=1400$ changed completely compared to $\mathrm{Re}=250$, Table 8. This confirms the preliminary assumptions raised in chapter 1. Contrary to observed for $\operatorname{Re}=250$, the difficulty to achieve JF $>1$ was not verified for $\operatorname{Re}=1400$ , according to assessments already reported by the literature. Common-flow-up configuration was not readily identified as the best choice for the JF objective function, according to conclusions presented for $\operatorname{Re}=250$, especially evaluating the VG position close to tube 2. In fact, the VG arrangement shown in Figure 33b was not directly recognized in Figure 87a. However, the flow acceleration through the nozzle created between VG and tube was identified and is later explored by Figure 97b. Thus, common-flow-up arrangement was the better choice, if the features from JF objective function are the main goal.

For $\mathrm{JF}^{\frac{1}{3}}$, some similarly with optimal configuration found to JF were observed, considering the best results from $D O$ procedure. The $V G_{2}$ arrangement was exactly the same for both and for $V G_{1}$ slightly changed. Again, this similarity was not observed for $\operatorname{Re}=250$. Evaluating only the literature works the configuration by Lemouedda et al. (2010) "Down" presented the better performance than Torii et al. (2002); this was also found for $\mathrm{Re}=250$ and $\mathrm{JF}^{\frac{1}{3}}$. The configuration found for the present work was completely different than that reported by Lemouedda et al. (2010), Figure 87b, and in Figure 84, the global results were considerably higher than from the open literature researches. Although the global results of the RSM approach was lower than DO approach, Figure 84, VG arrangement for RSM could be considered an excellent arrangement faced to arrangement from the literature researches. Nonetheless, the arrangement found by optimization procedure for DO approach cannot be considered as a global optimum. Based on literature results, commonflow-down arrangement is the best choice when the feature from $\mathrm{JF}^{\frac{1}{3}}$ objective function is the main goal, although the best results achieved by VG arrangement from present work (DO) are not like a common-flow-down configuration. Actually, this arrangement was akin to common-flow-up configuration, especially regarding to $\mathrm{VG}_{1}$ arrangement.

Figure 85 presents the global results considering the maximum Colburn factor j. RSM and DO approaches achieved the best results than those from the literature surveys. The results from optimization procedure were significantly higher than current 
researches, confirming the great advantage of the methodology proposed herein. For $\operatorname{Re}=1400$, differently than was observed for $\operatorname{Re}=250$, the VG arrangement for the objective function $\mathrm{j}$ was not similar to objective function $\mathrm{JF}^{\frac{1}{3}}$. However, as for $\mathrm{JF}^{\frac{1}{3}}$, among the works in open literature, Lemouedda et al. (2010) "Down" presented the best performance for the maximum heat transfer. Also, comparing only the literature survey works, common-flow-down configuration was the best choice if the heat transfer rate was the main goal; perceptibly, the pressure penalty to common-flowdown configurations was higher than to common-flow-up configuration. Result from DO approach for the objective function $\mathrm{j}$ was higher than RSM approach, Figure 85. However, an observation is related to associated pressure drop for each approach, ie, it could be expected that the maximum heat transfer would result in higher pressure drop. In fact, this was concluded since, in Figure 85, the RSM approach increased the pressure drop with lower heat transfer faced to DO approach. One cause for this is evidenced in Figure 88, which the main vortex produced by $V G_{1}$ was directed onto $V G_{2}$, according to RSM arrangement; this was not observed for DO arrangement. Still about this, both approaches generated longitudinal vortex in counter-clockwise, which indicates again that the root cause for this issue was due to difference between $\mathrm{y}_{1}$ and $\mathrm{y}_{2}$. In fact, for DO approach this difference was higher than RSM approach.

Regarding to pressure drop, evaluated in Figure 86, both optimization procedure (RSM and DO) found VG arrangement able to reduce the pressure loss. However, some literature researches were also able for this, such as: arrangement by Lei et al. (2010) and Torii et al. (2002). Moreover, these literature arrangements confirmed the trend observed for $\operatorname{Re}=250$, which showed that the common-flow-up configurations is the best choice to decrease the pressure drop. An important conclusion made for $R e=250$, mentioned that $V G_{1}$ was not necessary placed to decrease the pressure drop, because the VG was in wake region, behind the first tube. However, for $R e=1400, V G_{1}$ was placed in front of the first tube for both $R S M$ and DO approaches. At this moment, this question is on hold and is later explored in detail in this section. Among the current researches, Torii et al. (2002) showed the best result, which was very similar to Lei et al. (2010). 


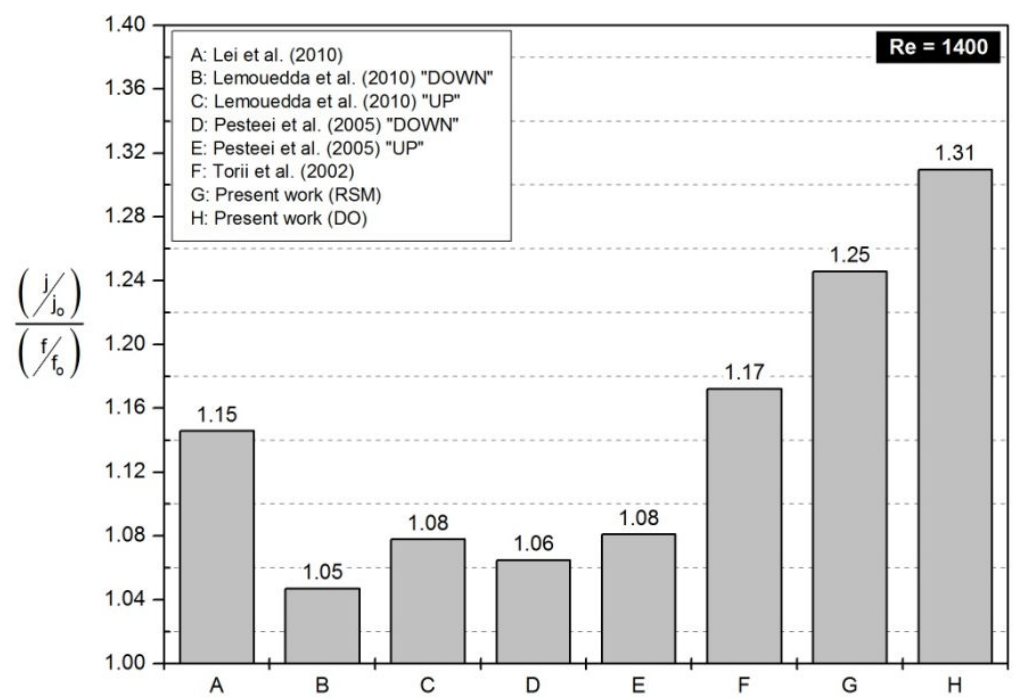

Figure 83 - Global results for JF objective function.

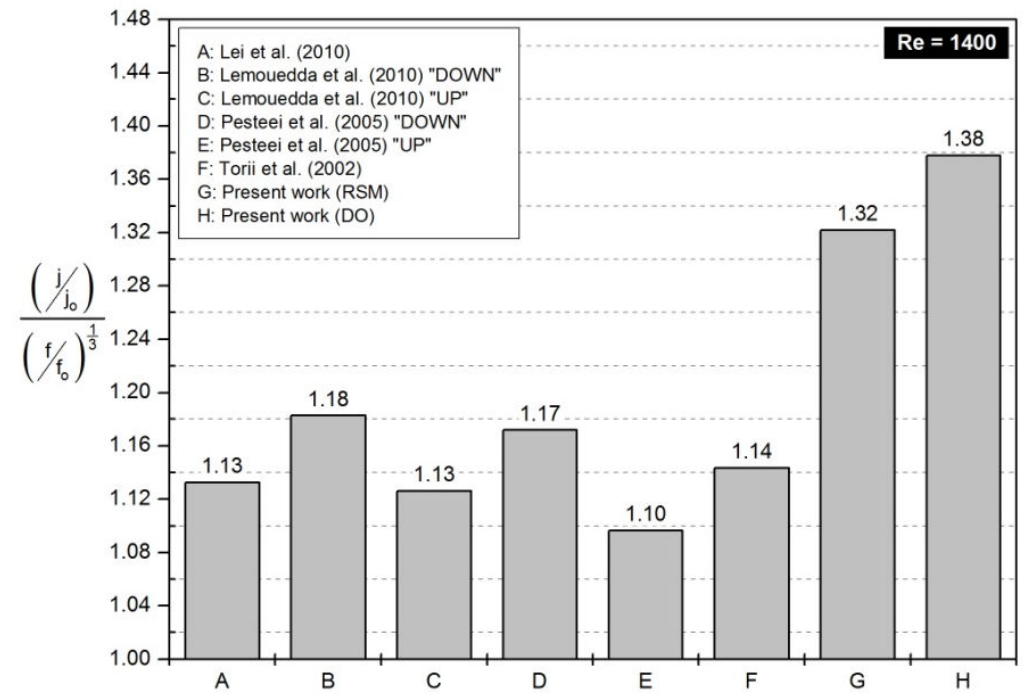

Figure 84 - Global results for $\mathrm{JF}^{\frac{1}{3}}$ objective function.

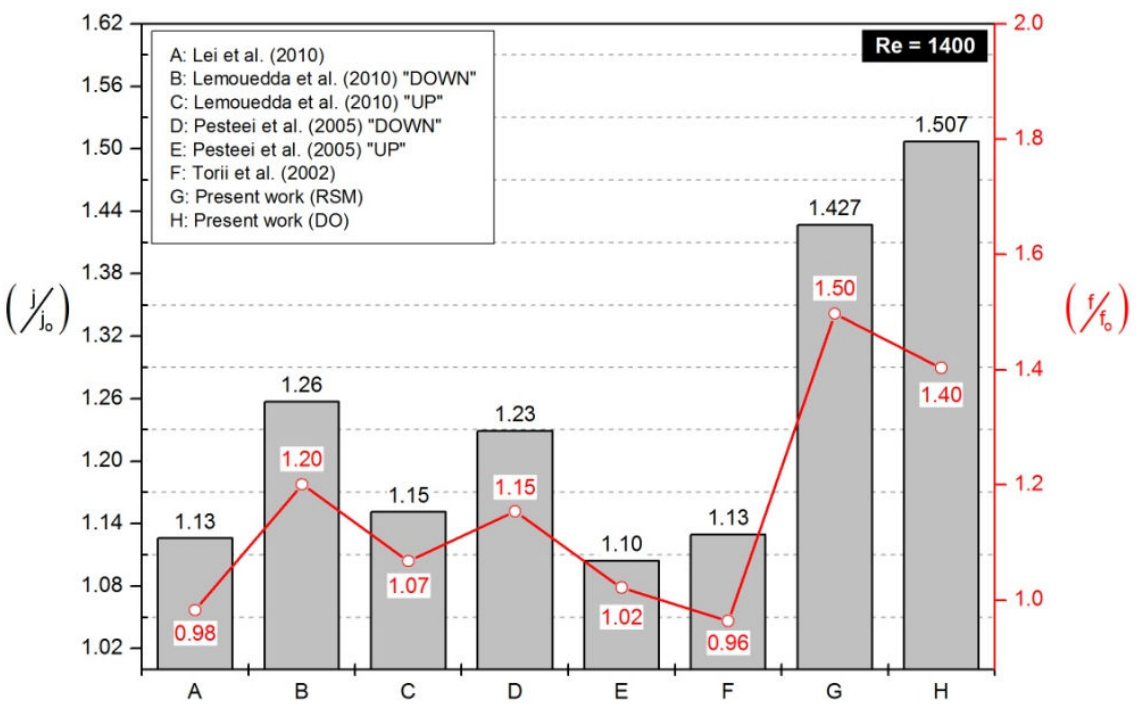

Figure 85 - Global results for j objective function with correspondent drop pressure penalty. 


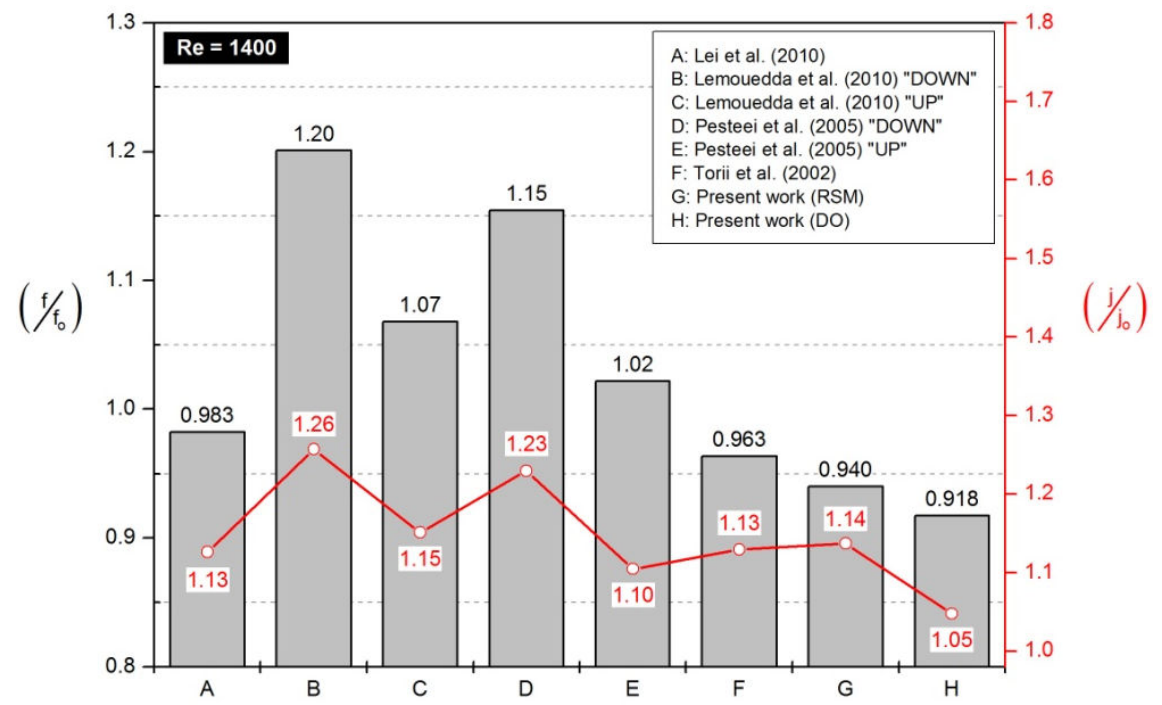

Figure 86 - Global results for $f$ objective function with correspondent augmentation heat transfer.

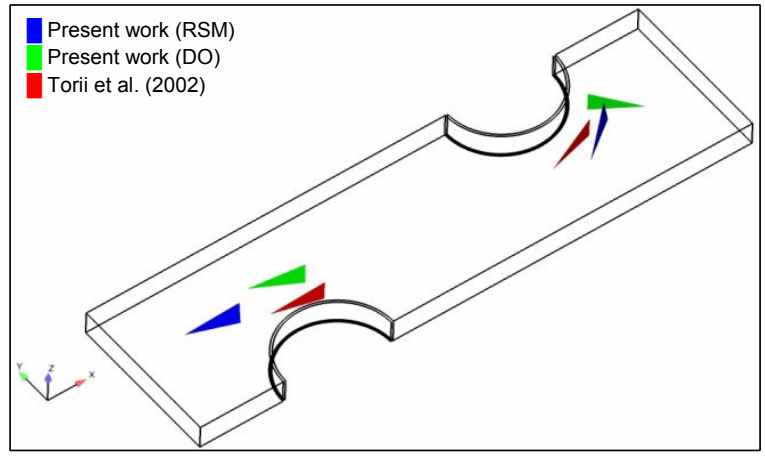

(a) JF

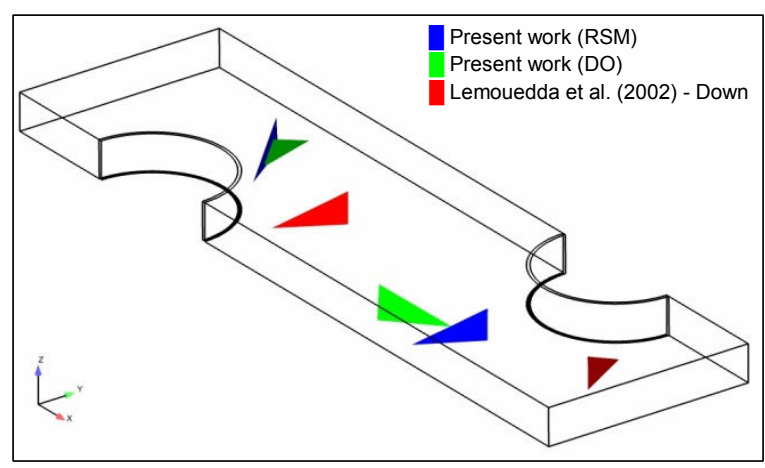

(c) Colburn factor, j

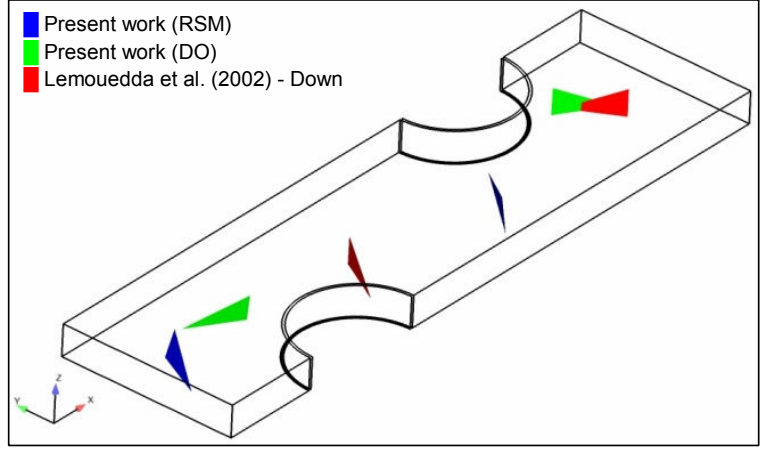

(b) $\mathrm{JF}^{\frac{1}{3}}$

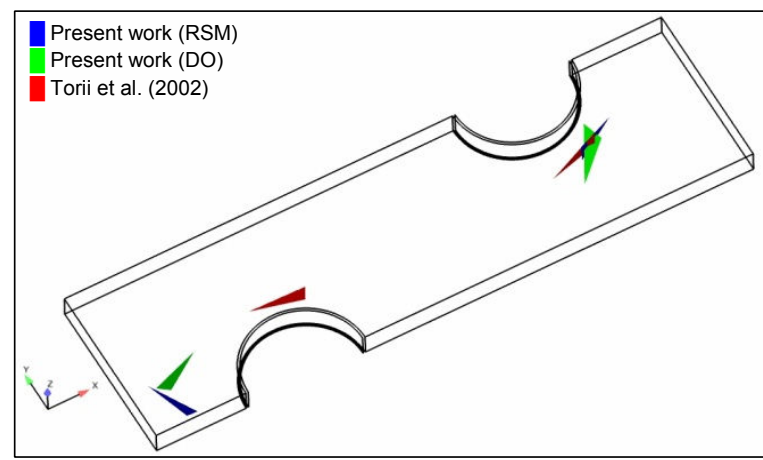

(d) Friction factor, $f$

Figure 87 - Overview of the position and angle for the best results from Figure 83 to Figure 86. 


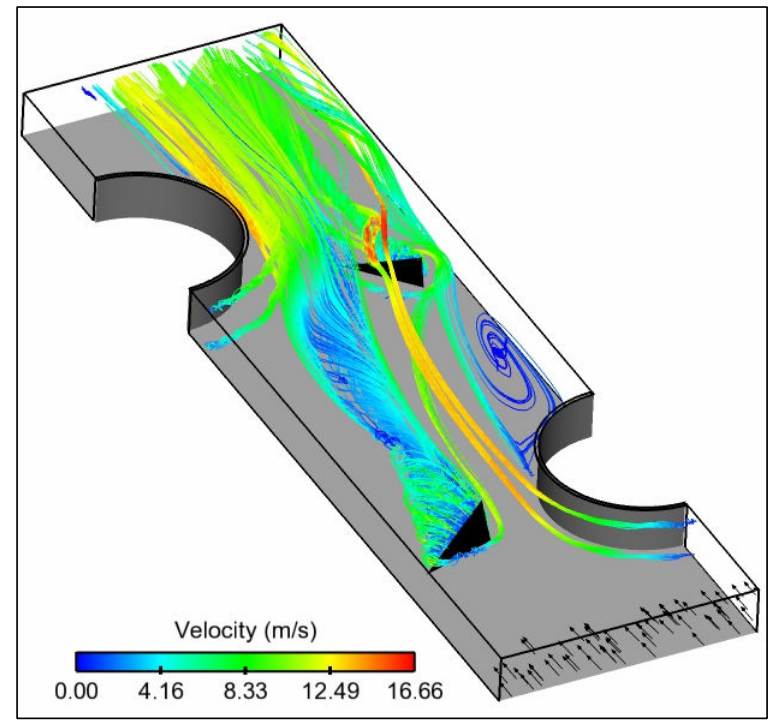

(a) DO approach.

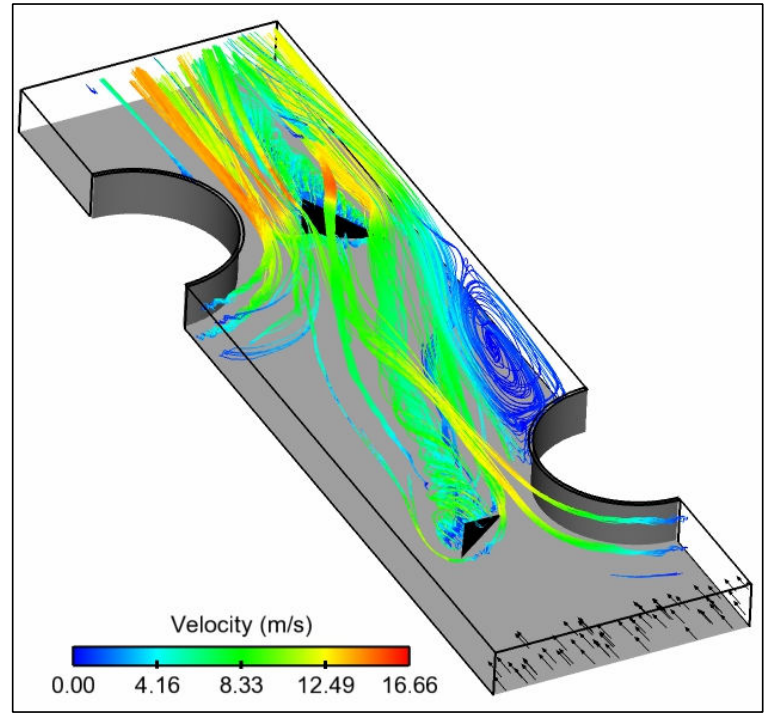

(b) RSM approach.

Figure 88 - Comparison between vortical structures by DO and RSM approach for objective function j.

Figure 89 to Figure 91 present the distribution of span-average Colburn factor along the fin length of the heat exchanger with and without VG, for each objective function, except to objective function $f$, which does not make sense for evaluating the Colburn factor when the objective function is to minimize the Friction factor. Thus, according to analysis from $\operatorname{Re}=250$, only the best results from Figure 83 to Figure 85 were plotted. Again, Fin-tube described at graphic legend relates to heat exchanger without vortex generator.

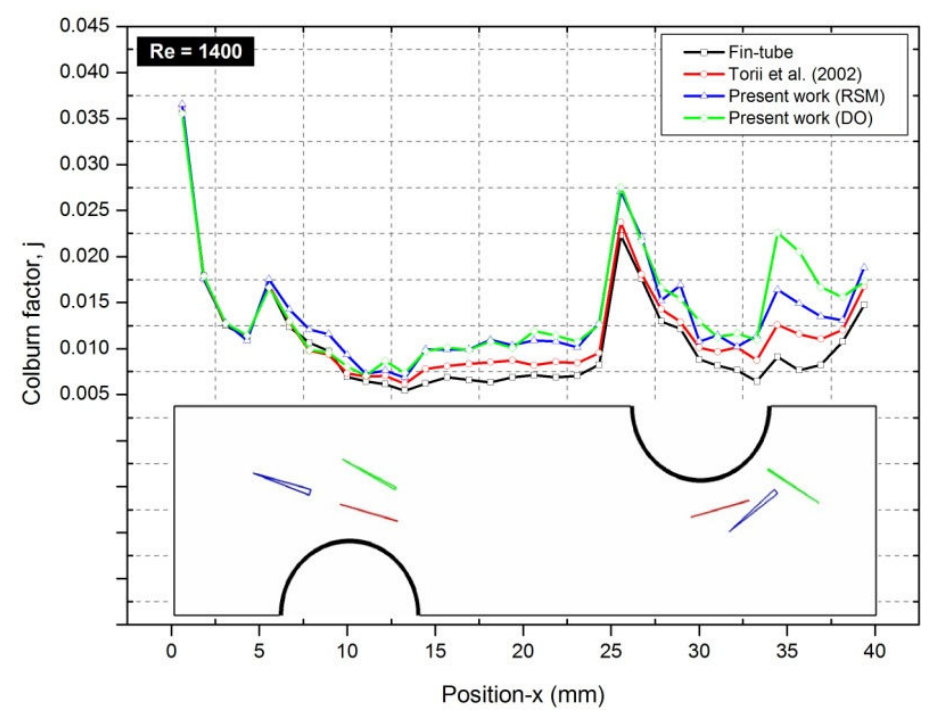

Figure 89 - Span-average Colburn factor (j) for the best results (Figure 83) for objective function JF. 


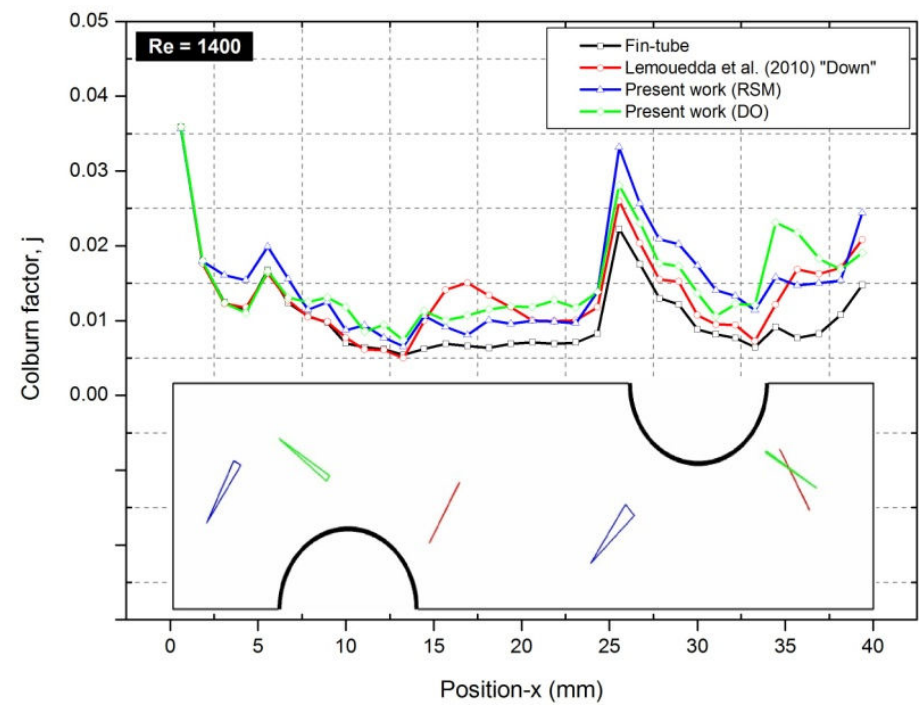

Figure 90 - Span-average Colburn factor (j) for the best results (Figure 84) for objective function $\mathrm{JF}^{\frac{1}{3}}$.

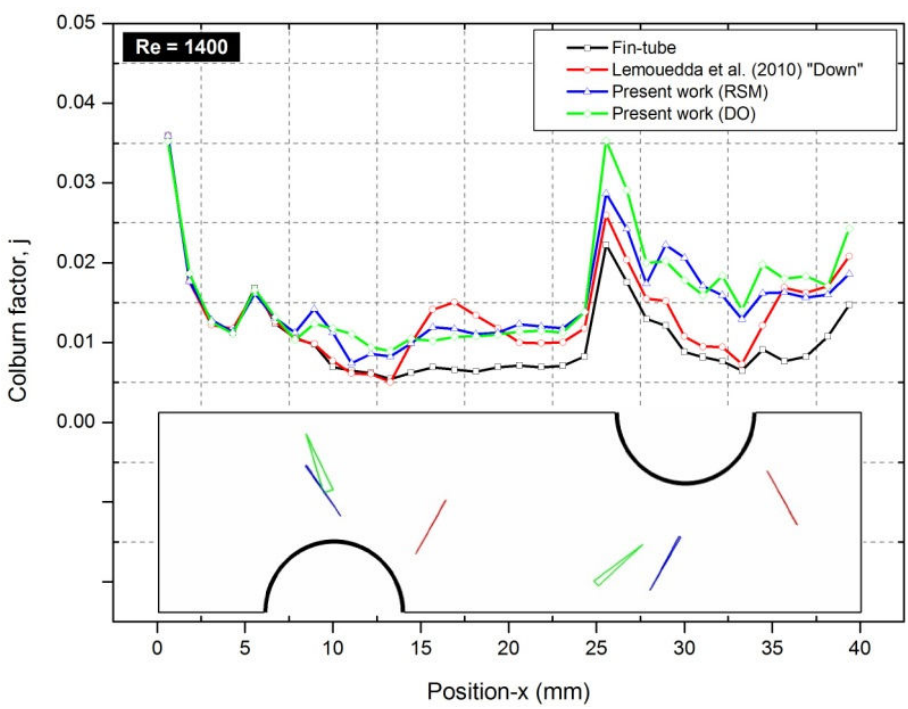

Figure 91 - Span-average Colburn factor (j) for the best results (Figure 85) for objective function j.

For both, Fin-tube and enhanced configurations, the distributions of the spanaverage Colburn factor presented similar behavior up to the place of the leading edge of the VG, as already expected and also verified for $\operatorname{Re}=250$. The common behavior described in previous section and mentioned by Lemouedda et al. (2010), for the VG applied on compact heat exchanger also for $\operatorname{Re}=1400$, the Colburn factor was very large at the inlet region where the temperature gradient is maximum at the leading edge of the fin bottom for the first time, and then both the velocity and thermal boundary layers develop along the flow direction that result in the decrease of heat transfer. In front of the first and second tube, the span-average Colburn factor 
abruptly increases due to the formation of horseshoe vortices, which provide better mixing and enhances heat transfer in this region. The values of the span-average Colburn factor for the enhanced configuration were higher than the Fin-tube. According to earlier mentioned, the VG creates an accelerating flow and secondary flow, which lead the stream flow into the wake region and enhance the mixing of the hot and cold fluid. This is the reason for local Nusselt number behind the delta winglet was greatly improved. This was observed in Figure 89 to Figure 91, evaluating the Colburn factor profile behind the tube 2 and more evident when is evaluated the relation between enhanced configuration and Fin-tube. These results are later explored from Figure 92 to Figure 94.

Evaluating the objective function JF, Figure 89, along the flow direction, the results for the present work was higher than the survey literature work (Torii et al., 2002) for both RSM and DO approaches. The greater difference between the present work and Torii et al. (2002) was exactly after the VG on first and second tube. This difference was more pronounced on the second tube, close to the wake region behind the tube, where fluid recirculation with low velocity causes poor heat transfer; the attack angle was fundamental to achieve better mixture between cold and hot fluid. A significant increase on local Colburn factor was noted behind the tube 2 for arrangement identified by present work (DO), which the VG configuration is unusual and was not found in literature review. Close to first tube, the Colburn factor profile by present work (RMS) arrangement was higher than present work (DO) arrangement, which could suggests that it would be the best choice. However, the local pressure loss for RSM arrangement was significantly higher than DO arrangement, which impacted a lot on objective function JF, as already discussed.

Figure 90 presents the span-average Colburn factor for objective function $\mathrm{JF}^{\frac{1}{3}}$ with the same trends of the results for the objective function JF, ie, there is a significant augmentation heat transfer after the VG, which is more pronounced for the present work, especially for the DO approach on the second tube. Close to tube 1, enhancement heat transfer from Lemouedda et al. (2010) was locally higher than the present work. However, a local higher pressure loss due to vortex generator was observed, which is not desired for $\mathrm{JF}^{\frac{1}{3}}$. Anyway, arrangement found by RSM also seems to be interesting since global result, Figure 84, was higher than literature 
researches. Between RSM and DO approaches, the position of the $V G_{2}$ was decisive considering that the VG arrangement for DO approach was disposed closer to wake region, behind the tube 2, whereas the RSM approach was superior to DO approach in front of the tube 2. However, the major difference between them was the pressure drop immediately after the $V G_{2}(R S M)$, increasing the global pressure drop evaluated in the outlet domain. Also, $\mathrm{VG}_{2}$ arrangement by present work (DO) was placed further upstream adjacent to the tube in order to create a nozzle-like flow passage, which provide separation delay and narrows in the wake; this assessment is explored later.

Figure 91 shows the span-average Colburn factor for the objective function j. After each vortex generator, it was noted significant augmentation in the heat transfer faced to Fin-tube. Close to tube 1, span-average Colburn factor, considering arrangement by Lemouedda et al. (2010), was locally higher than both present work (RSM and DO); however, the longitudinal vortex was less persistent than present work arrangement. This shows that the best position for $V_{G_{1}}$, to achieve maximum heat transfer rate, is close to minimum flow area, which could generate stronger longitudinal vortex, defined for the pressure difference between the main flow and the back-side of the $V G_{1}$. This minimum flow area is the position where was verified the maximum average velocity. This same conclusion was made for $\operatorname{Re}=250$. Obviously, the input variables $\mathrm{y}_{1}, \theta_{1}$ and $\varphi_{1}$ were adjusted by optimizer for each application, according to Table 8. Close to the second tube, the more pronounced enhancement heat transfer to the present work was due to VG location, which is placed close to wake region behind the tube 1 , causing the fluid mixture in wake region (knowledge by lower heat transfer coefficient), and almost at minimum flow area due to the same features already discussed. Another important feature was the interaction between the vortices generated by VG, which is evaluated later through views applying the methodology called by Core Vortex. Between $\operatorname{Re}=1400$ and $\operatorname{Re}=250, V_{2}$ arrangement by present work was not similar, inducing to conclusion that the $V G_{2}$ does not impact on the tube 2. However, due to higher velocity for $\mathrm{Re}=1400$ and, although the distance between $V_{G_{2}}$ and tube 2 is higher, separation delay on tube 2 was noted, which could direct the flow into wake region behind the tube 2. 
Following the same mind-set of analysis applied for $\operatorname{Re}=250$, it is evaluated the relation between enhanced and Fin-tube configurations along the flow direction. Reminding, the main goal of this is let us identify the region which is more enhanced, indicating where the visualization step should be focused. Figure 92 to Figure 94 present these results and indicated some trends already discussed in Figure 89 to Figure 91, evidencing some conclusions already made and explaining several others.

For both objective functions the relation of the Colburn factor was more enhanced after the VG, remarkable behind the second tube and close to vortex generator arrangement by Lemouedda et al. (2010) for objective function $\mathrm{j}$ and $\mathrm{JF}^{\frac{1}{3}}$. For $\operatorname{Re}=1400$, augmentation heat transfer along the fin was higher than $\operatorname{Re}=250$, especially in the region between the tubes. This was a consequence of the higher velocity for $\operatorname{Re}=1400$, which generated longitudinal vortex more persistent downstream. Figure 92 and Figure 93 indicated that the great difference between enhanced configuration and Fin-tube occurred behind the second tube, notoriously by the present work (DO). Both VG arrangements were equals and, even more interesting, is the fact that the optimization procedures were started with different initial population. This suggests great robustness of the methodology. Figure 94 points out increases in heat transfer after the VG arrangement by Lemouedda et al. (2010) and it was not observed significant increase in local pressure loss faced to arrangement by present work (DO). This is evidenced later in this section. VG position in direction- $x$ was the root cause for this result. However, a consequence of this is the generation of weaker longitudinal vortex, assessment made evaluating the span-average Colburn factor after the $\mathrm{VG}_{1}$, Figure 94 . This same behavior was also noted for objective function $\mathrm{JF}^{\frac{1}{3}}$, Figure 93. This root cause sounds as reasonable evaluating the span-average Colburn profile to Torii et al. (2002), Figure 92. In fact, $V_{G_{1}}$ arrangement by Torii et al. (2002) was closer to minimum flow area than arrangement by Lemouedda et al (2010), which was more flat along of the fin. 


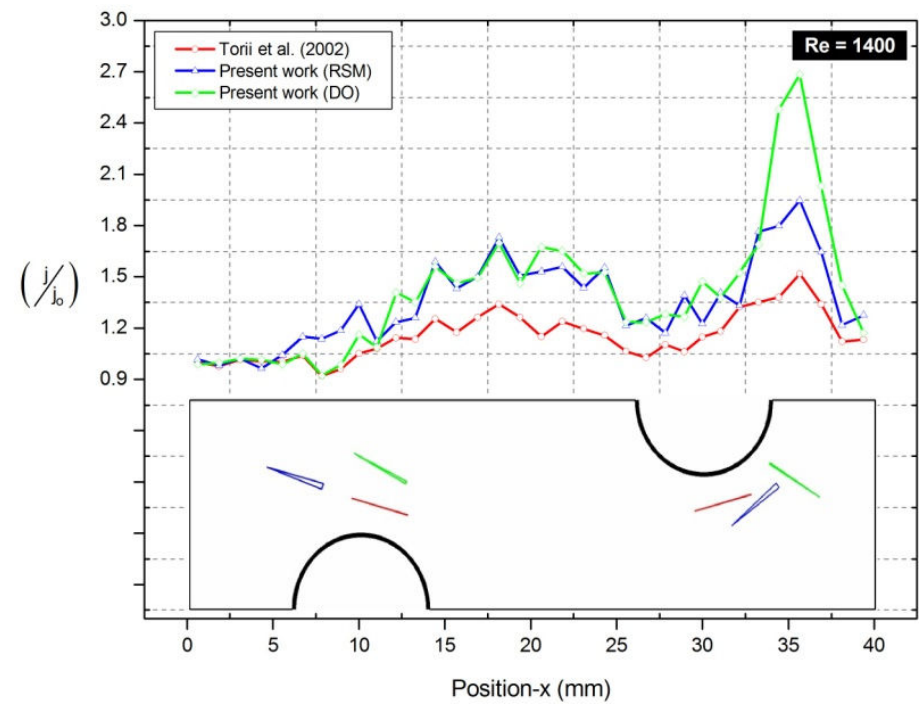

Figure 92 - Objective function JF. Span-average Colburn factor (j) for the best results (Figure 83).

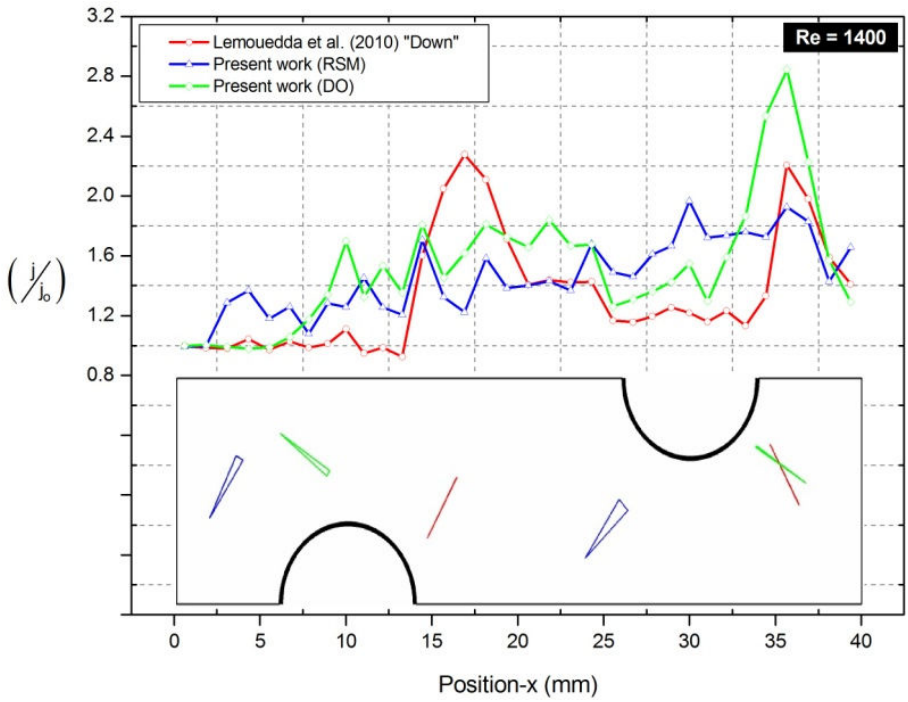

Figure 93 - Objective function $\mathrm{JF}^{\frac{1}{3}}$. Span-average Colburn factor (j) for the best results (Figure 84).

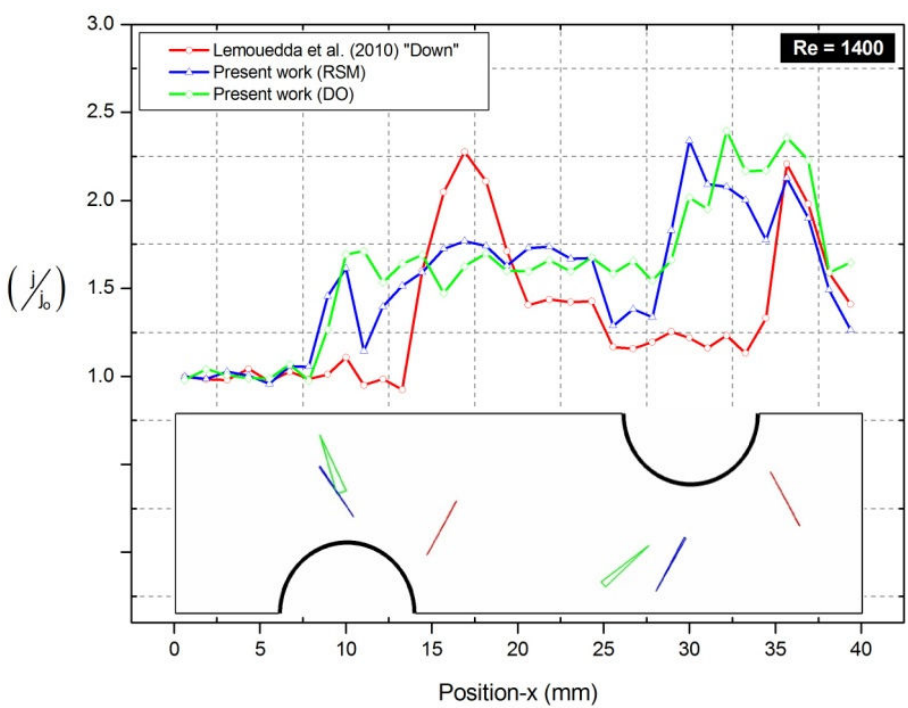

Figure 94 - Objective function j. Span-average Colburn factor (j) for the best results (Figure 85). 
Vortex Core (together with streamlines) and Flow Separation methodologies were already presented and were also applied to $\operatorname{Re}=1400$. Also, the next analysis were separately evaluated for each objective function, since the optimization procedure found different vortex generator arrangement and, as possible as, was provided a qualitative comparison between $\operatorname{Re}=1400$ and $\operatorname{Re}=250$. Additional to outlined post-processing methodologies, it was also presented the velocity plane at axis $\mathrm{z}$ along of the flow direction to evidence the outcomes discussed so far. Providing adequate comparison between $\operatorname{Re}=1400$ and $\operatorname{Re}=250$, the planes positions at axis $z$ were defined according to $\operatorname{Re}=250$, ie, at $z=0.5 \mathrm{~mm}, z=1.0 \mathrm{~mm}$ and $z=1.5 \mathrm{~mm}$.

Figure 97a and Figure 109a are for the same configuration by Torii et al. (2002). However, Figure 109a was repeated there to suit its results for each objective function, since Torii et al. (2002) was the best literature survey result found for both JF and $\mathrm{f}$. This was also considered for objective function $\mathrm{JF}^{\frac{1}{3}}$ and $\mathrm{j}$, which Lemouedda et al. (2010) was the best literature survey results, Figure 102a and Figure 106a. This was necessary to allow an additional qualitative evaluation by velocity field color range. Figure 95 to Figure 99 present results for the objective function JF, according to Figure 92. These set of analysis were jointly necessary to understanding the thermal-hydraulic results of the VG along of the direction flow.

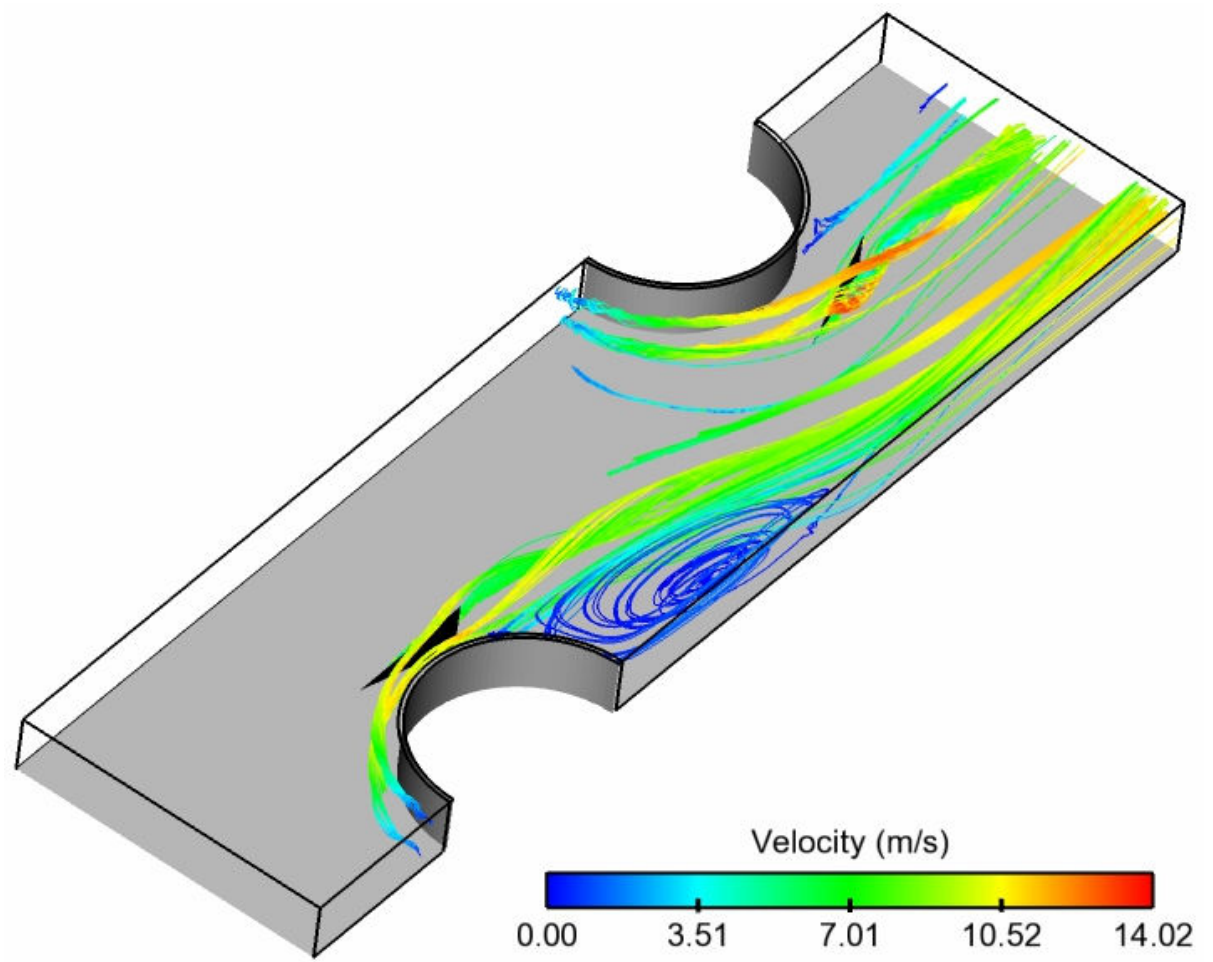

Figure 95 - Streamlines emitted from Core vortex by Torii et al. (2002) arrangement. 


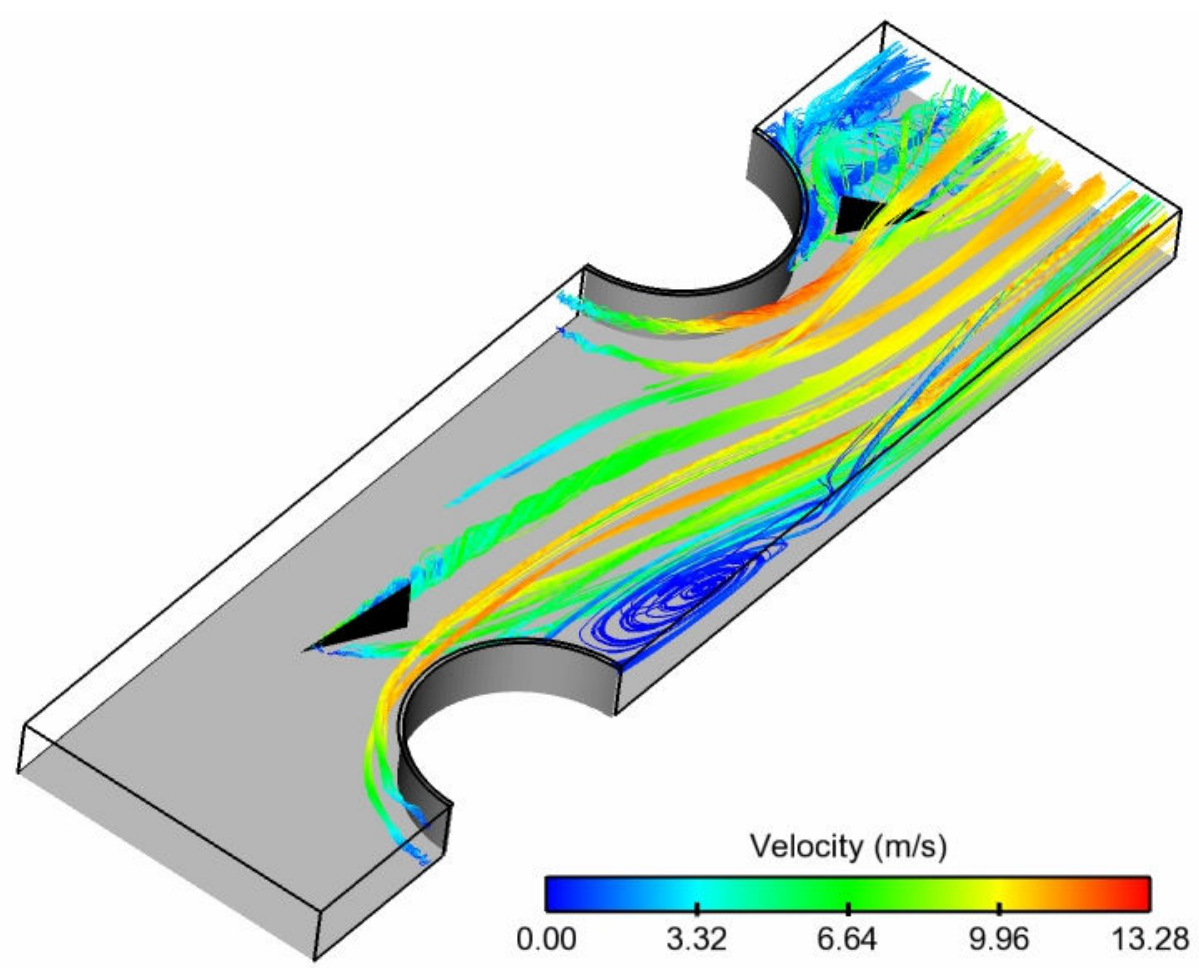

Figure 96 - Objective function JF. Streamlines emitted from Core vortex by present work (DO).

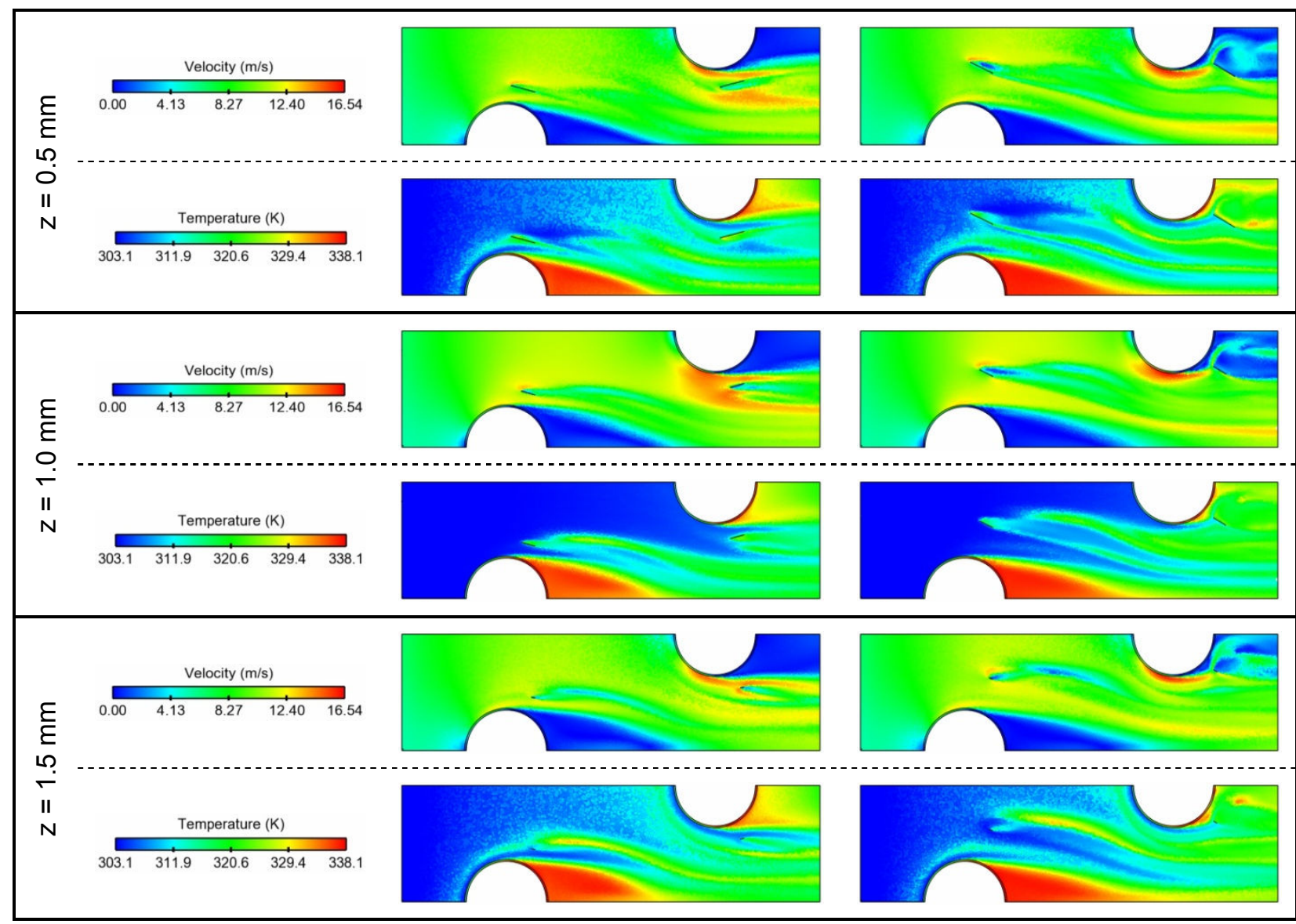

(a) Torii et al. (2002).

(b) JF present work (DO).

Figure 97 - Velocity and temperature field planes at axis-z, according to Figure 83. 


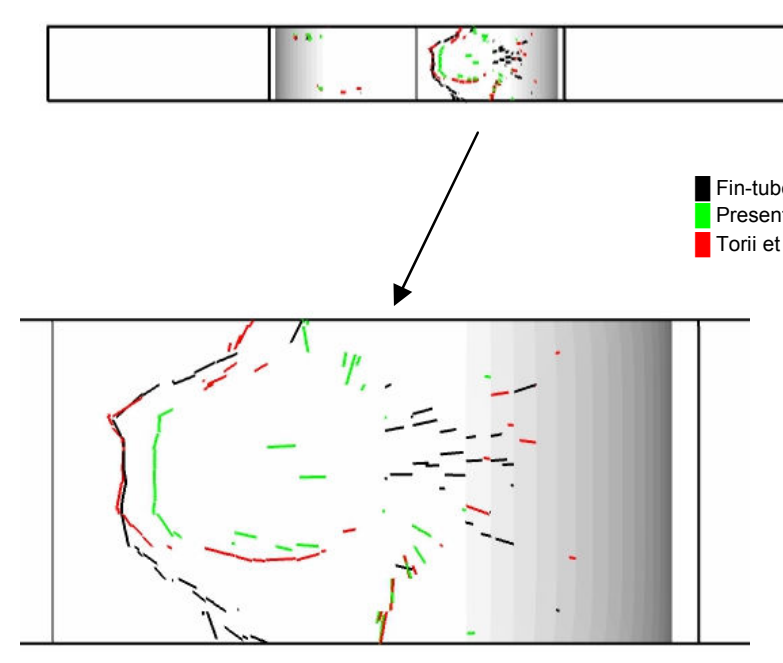

(a)

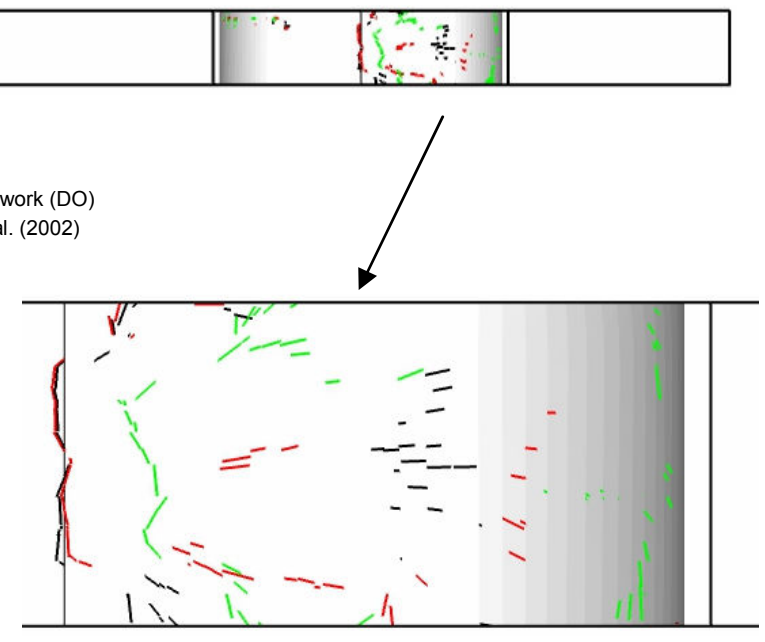

(b)

Figure 98 - Objective function JF. Flow separation profile on (a) tube 1, (b) tube 2.

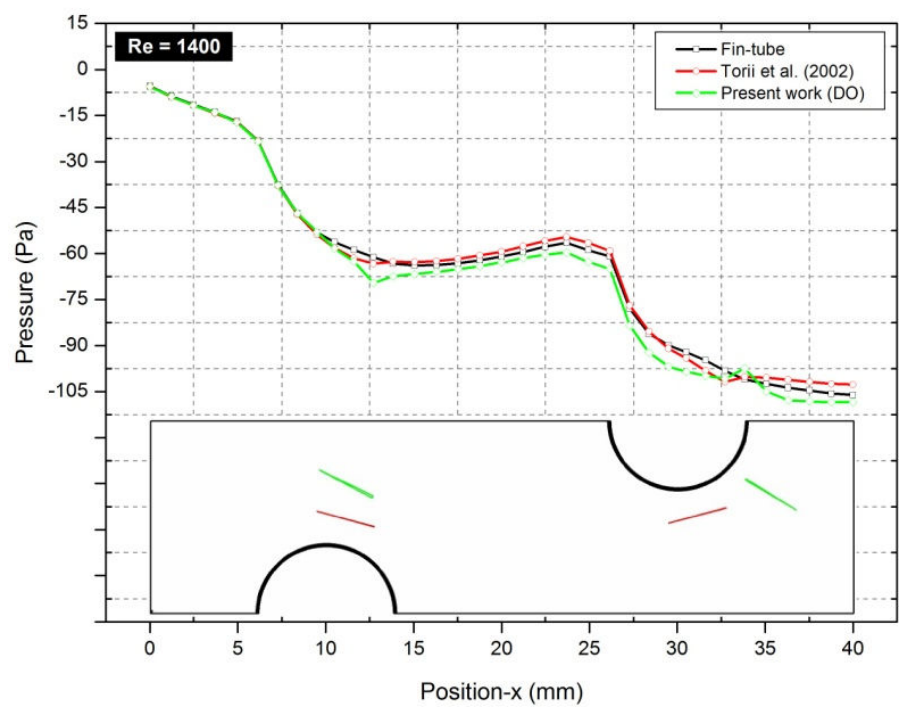

Figure 99 - Pressure profile considering the objective function JF.

Figure 95 and Figure 96 show the streamlines emitted from core vortex considering the configuration by Torii et al. (2002) and by the present work, through direct optimization (DO), respectively. Both configurations were able to produce secondary flow, which increased the local heat transfer coefficient. For both, the main vortex produced by $V G_{1}$ was not directed onto $V G_{2}$, although the vortices are persistent along of the flow direction, contributing to decrease the global pressure drop. Vortex generator arrangement by Torii et al. (2002) causes slightly separation delay on the tube 1 and tube 2, Figure 98, due to flow acceleration between VGs and tubes, 
especially at $z=0.5 \mathrm{~mm}$, Figure 97 . Consequently, the local pressure drop in these regions was decreased compared to Fin-tube, Figure 99. By present work (DO), was verified more pronounced separation delay on the tube 1 and tube 2 than Torii et al. (2002), Figure 98. However, the local pressure drop was higher than Fin-tube, which confirms some assessments already discussed, ie, separation delay contribute to decrease the global pressure drop, but this is not the core to achieve local pressure drop. In fact, separation delay was more pronounced for the present work (DO) than Torii et al. (2002), Figure 98, although the pressure drop was higher, Figure 99. In this case, attack angle of the VG for the present work (DO) was crucial for this, although the $V \mathrm{~V}_{1}$ was not placed close to tube 1. Corner vortex also contributed for the separation delay, beyond increase local heat transfer rate. For the second tube, the separation delay was more pronounced for the present work (DO) than Torii et al. (2002), Figure 98, which is a consequence of the flow acceleration at nozzle created between $V G_{2}$ and tube 2, Figure 97. This flow was led to region where the heat transfer coefficient is low, resulting in higher fluid mixing and consequently a stronger heat transfer between the fluid and the plate-fin wall. This assessment explains the results shown in Figure 92. Vortex generator arrangement was difference for $\operatorname{Re}=1400$ and $\operatorname{Re}=250$, ie, while $V G_{1}$ was placed in front of the first tube for $\operatorname{Re}=250$, for $\operatorname{Re}=1400$ was placed close to the minimum flow area. In fact, for $\operatorname{Re}=250$ the air flow velocity was lower than $\operatorname{Re}=1400$ and, therefore, the air temperature in the outlet domain for $\operatorname{Re}=250$ was higher than $\operatorname{Re}=1400$, ie, the secondary flow provide by $V_{G_{1}}$ could be more to increase the global pressure drop than to increase the heat transfer rate especially, for $\operatorname{Re}=250$. This is one of the root causes that are not easy to enhancement heat transfer at low velocity. Thus, for $\mathrm{Re}=250$ and for objective function JF, there is no needs of the secondary flow is so persistent on downstream, which explain the position found by optimizer in front of the tube 1, away from the minimum flow area (maximum velocity). For $\operatorname{Re}=1400$, it was evident the need of stronger secondary flow, explaining the position found by optimizer close to the minimum flow area. For the second tube, the separation delay was more pronounced by the present work (DO) arrangement than Torii et al. (2002) arrangement. However, this effect just slightly mitigates the pressure drop profile, according to Figure 99, evaluated in the outlet domain. This separation delay was due to the flow acceleration behind the second tube, according to Figure 97 . Still considering results from Figure $97, V G_{2}$ arrangement caused great flow mixture in 
the wake region, justifying the abrupt heat transfer enhancement close to tube 2 identified in Figure 92. Differently that observed for low Reynolds number, for $\operatorname{Re}=1400$, the main vortex generated by $V G_{1}$ and $V G_{2}$ were counter-clockwise, while for Torii et al. (2002) was counter-clockwise for $V G_{1}$ and co-clockwise for $V_{2}$. These different patterns were also justified due to symmetry of the VGs for Torii et al. (2002), and the lack thereof for the present work (DO).Therefore, as already concluded for $\operatorname{Re}=250$, clockwise seems to be a secondary subject compared to relative position between VGs and tubes also to high Reynolds number.

Figure 100 to Figure 104 are related to objective function $\mathrm{JF}^{\frac{1}{3}}$ according to Figure 93 .

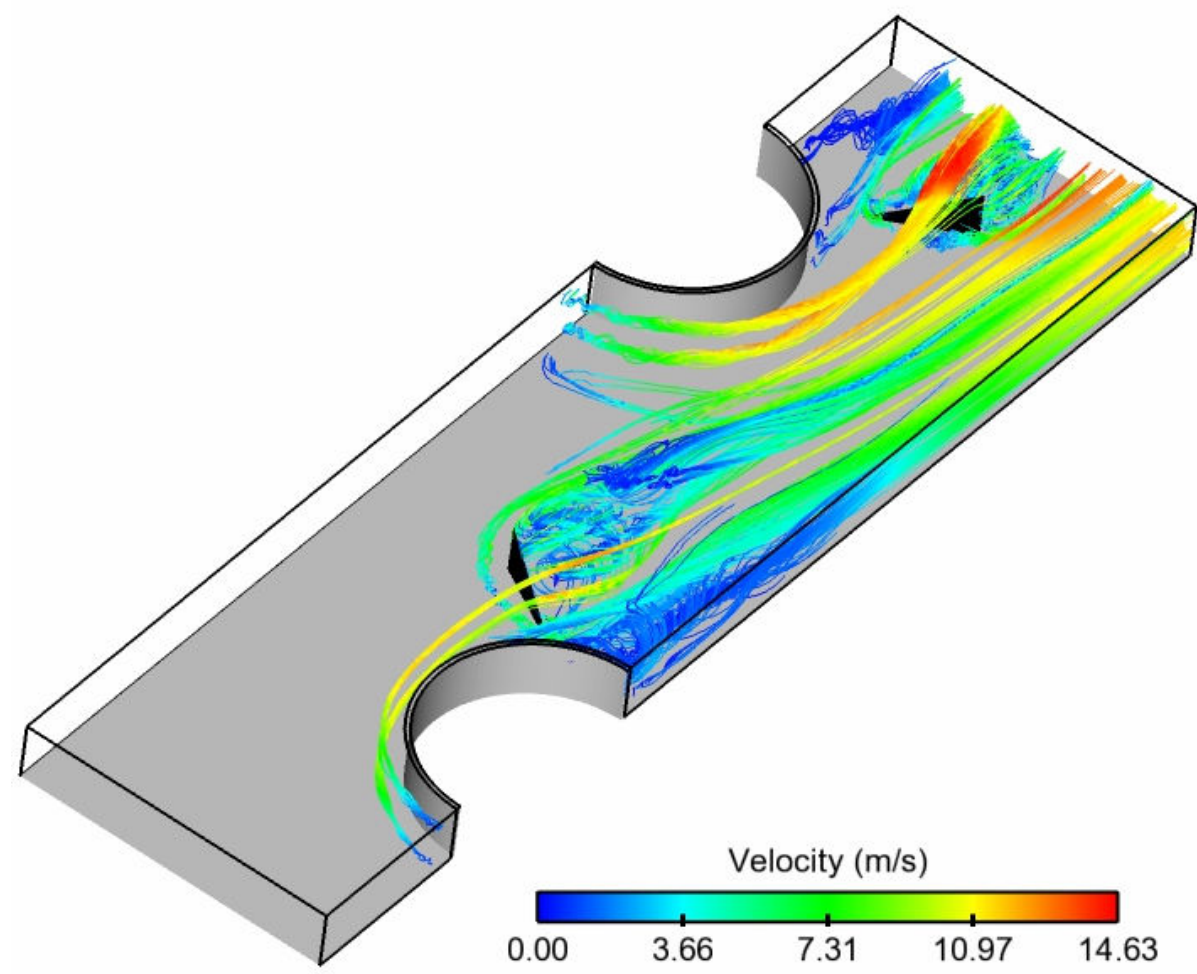

Figure 100 - Streamlines emitted from Core vortex by Lemouedda et al. (2010) arrangement. 


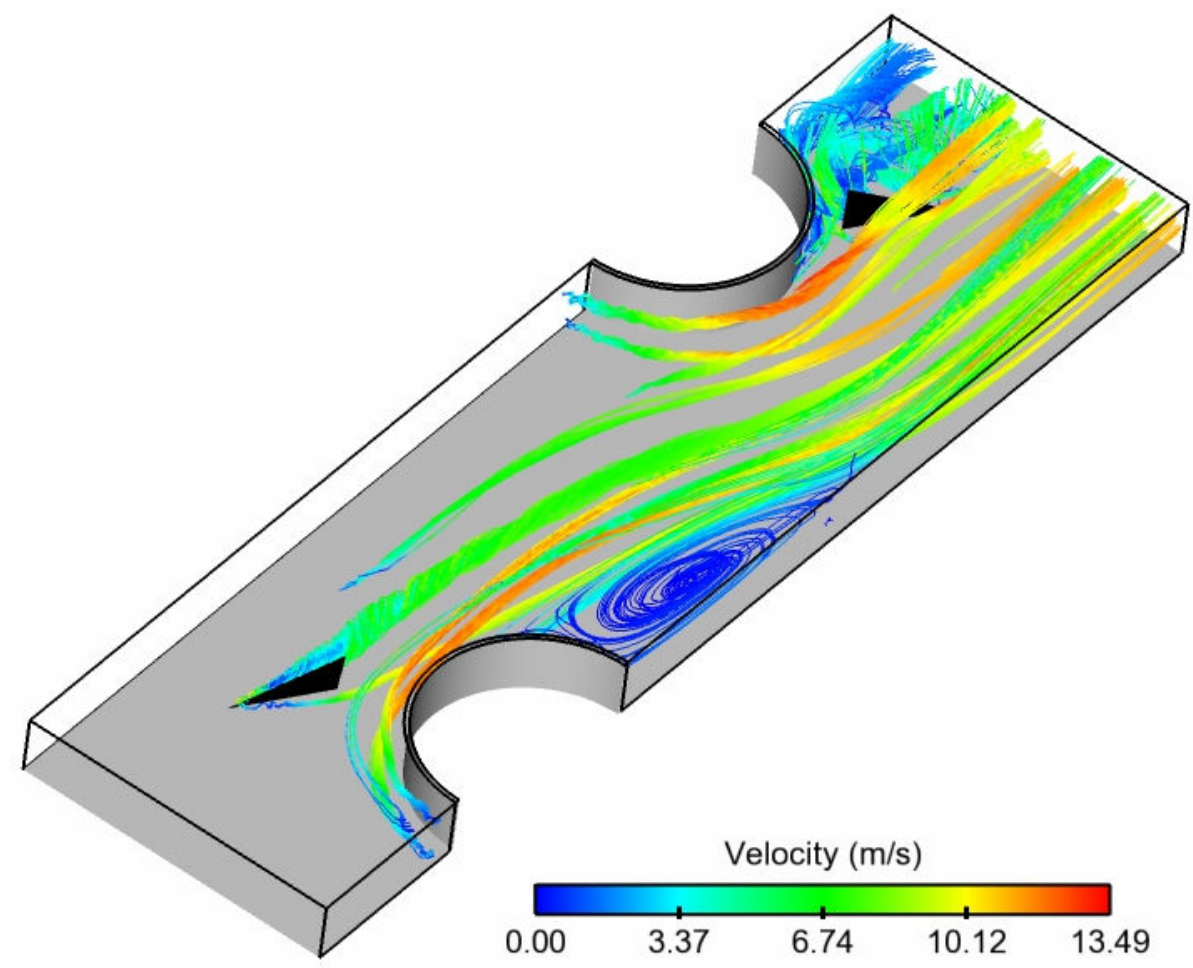

Figure 101 - Objective function $\mathrm{JF}^{\frac{1}{3}}$. Streamlines emitted from Core vortex by present work (DO).

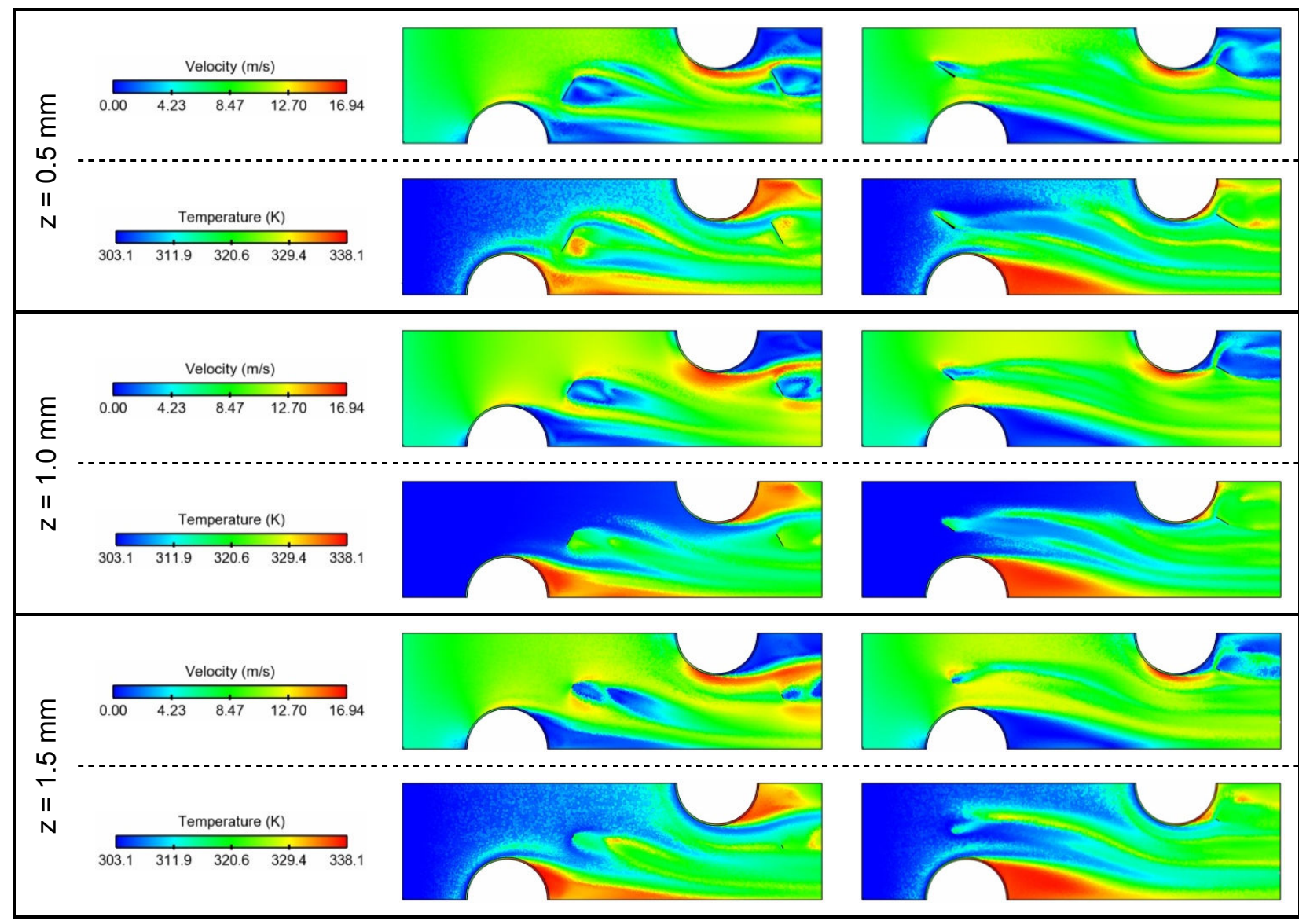

(a) Lemouedda et al. (2010).

(b) $\mathrm{JF}^{\frac{1}{3}}$ present work (DO).

Figure 102 - Velocity and temperature field planes at axis-z, according to Figure 84 . 


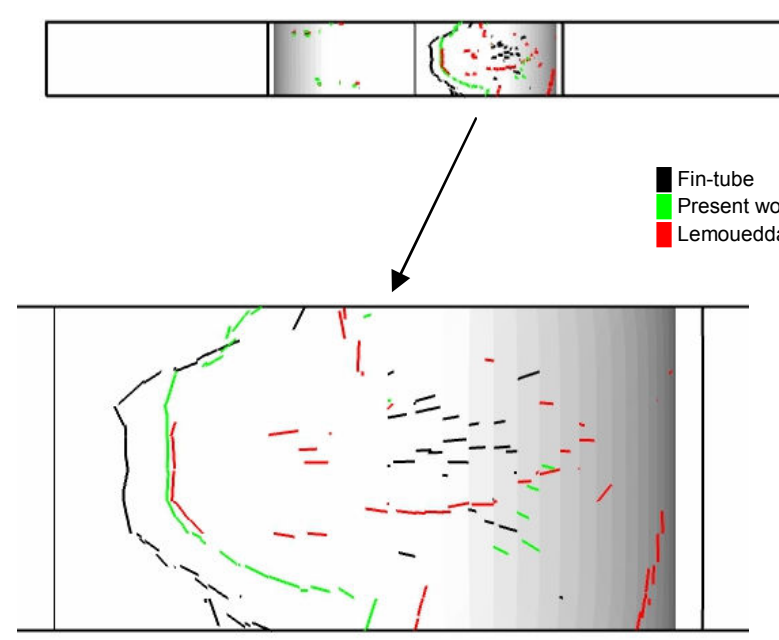

(a)

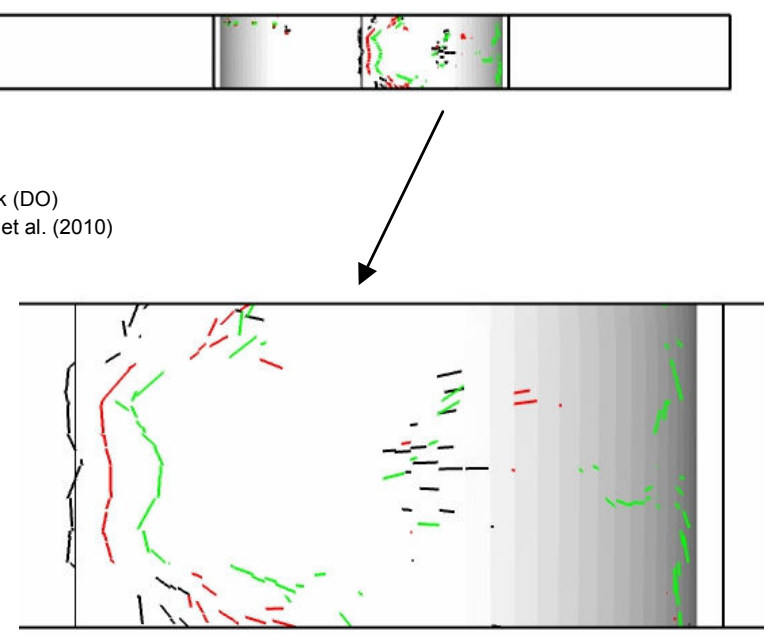

(b)

Figure 103 - Objective function $\mathrm{JF}^{\frac{1}{3}}$. Flow separation profile on (a) tube 1, (b) tube 2.

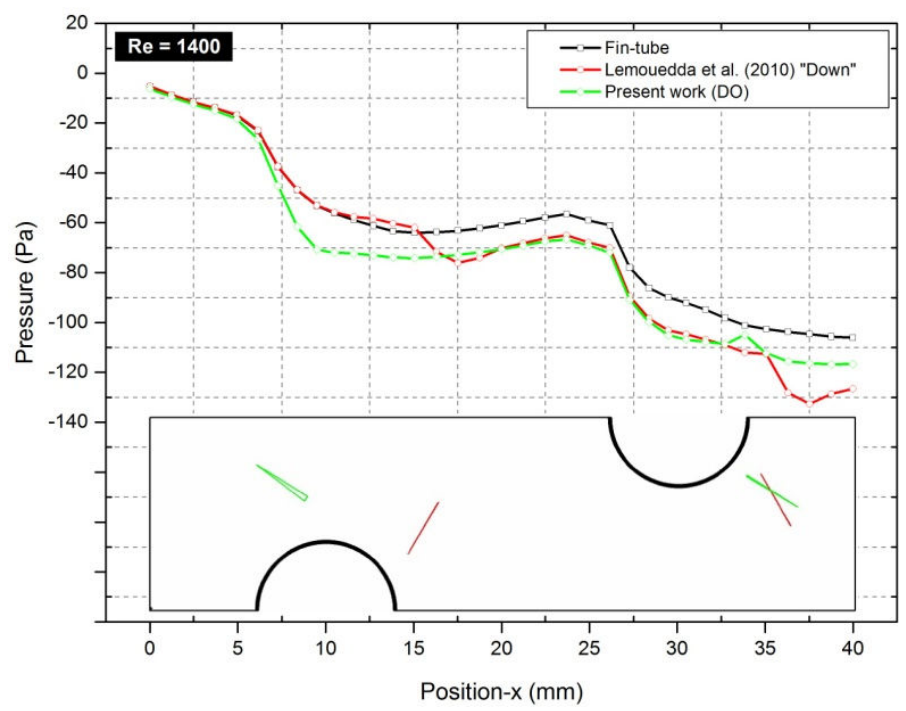

Figure 104 - Pressure profile considering the objective function $\mathrm{JF}^{\frac{1}{3}}$.

Figure 100 and Figure 101 show the streamlines emitted from the core vortex considering the configuration by Lemouedda et al. (2010) and by the present work, through direct optimization (DO), respectively. Both VG arrangements were able to produce secondary flow, Figure 100 and Figure 101, increasing the heat transfer coefficient. For both, the main vortex produced by $V G_{1}$ was not directed onto $V_{2}$, which also contributed to minimize the pressure drop. Separation delay on tube 1, was very similar for both configurations faced to Fin-tube, cooperating to mitigate the 
pressure drop. However, the pressure profile, reported in Figure 104, showed that the separation delay was not sufficient to mitigate the local pressure loss up to Fintube level. In this case, as already discussed, the VG position in direction- $x$ was the central point because the VG by the present work (DO) arrangement was placed close to the minimum flow area (maximum velocity), which cause flow acceleration and, consequently, the higher local pressure drop. This mentioned flow acceleration was evidenced analyzing the Figure 102b. For Lemouedda et al. (2010) arrangement, the mentioned separation delay induced slightly local pressure drop reduction faced to Fin-tube, Figure 104. On the second tube, both configurations also were able to produce separation delay, however, for the present work (DO), more pronounced due to flow acceleration evidenced in Figure 102 was noted. In fact, flow acceleration is clear at all level at axis-z and, as consequence of this, the flow mixture behind the second tube was increased, enhancing the heat transfer coefficient in this region. Thus, air flow directed toward the wake region explains the results showed in Figure 93. Moreover, the more pronounced separation delay for the present work (DO) on the second tube, explains the pressure profile in Figure 104 faced to Lemouedda et al. (2010). Comparing this to Fin-tube pressure profile, the separation delay was not sufficient to take this to Fin-tube level, because the history of the flow is essential when pressure field is analyzed. Therefore, the separation delay analysis of the net effect produced for each VG ought to be separately conducted for each tube. Main vortex rotation produced by $\mathrm{VG}_{1}$, according to Lemouedda et al. (2010) configuration, was co-clockwise and counter-clockwise for $V G_{2}$, due to symmetry between $V G$ s. For the present work (DO), $V G_{1}$ and $V G_{2}$ generated vortices in counter-clockwise. Therefore, as already concluded during this section, clockwise is a secondary subject compared to relative position between VG and tubes.

Figure 105 to Figure 108 are related to objective function j according to Figure 94. 


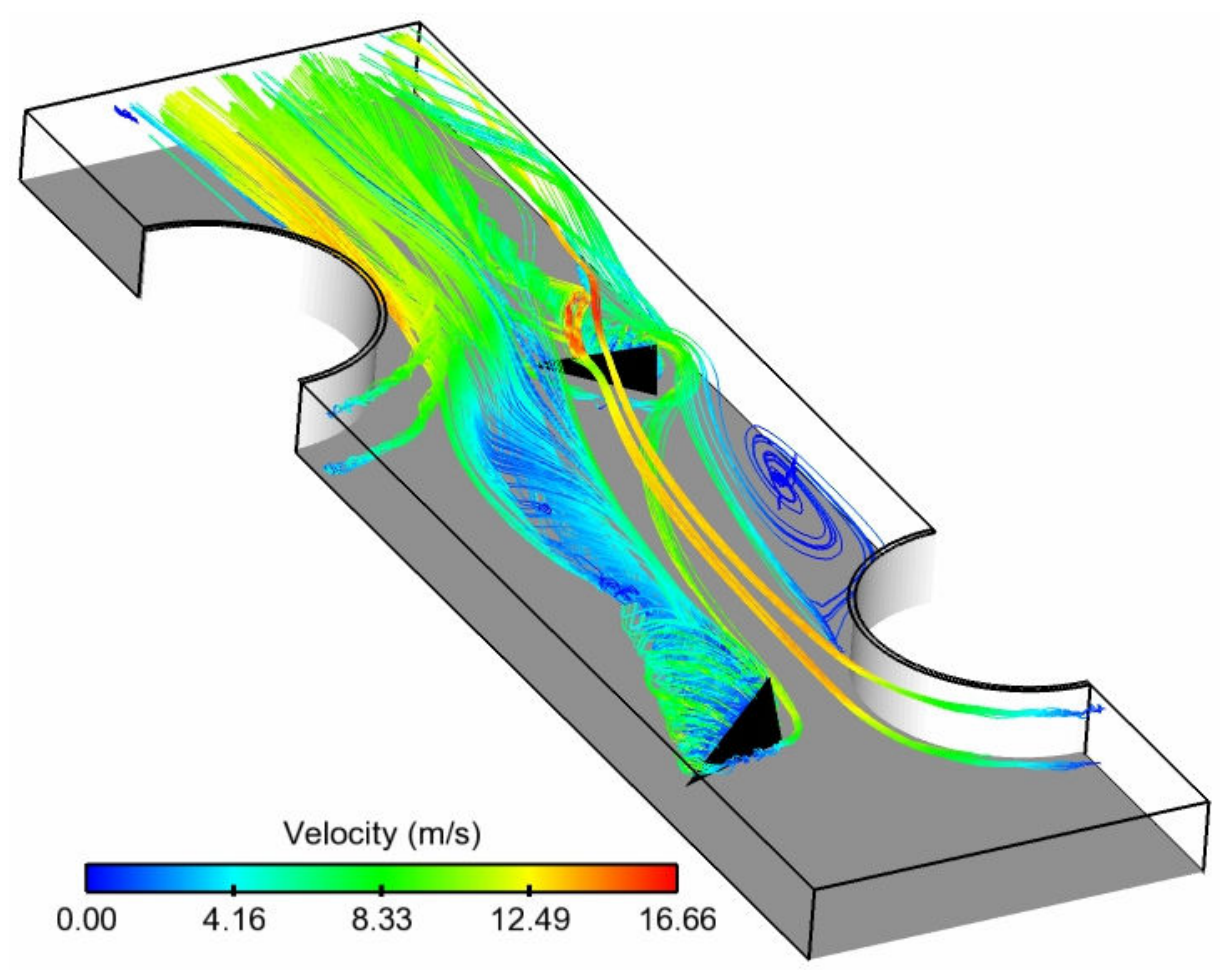

Figure 105 - Objective function j. Streamlines emitted from Core vortex by present work (DO).

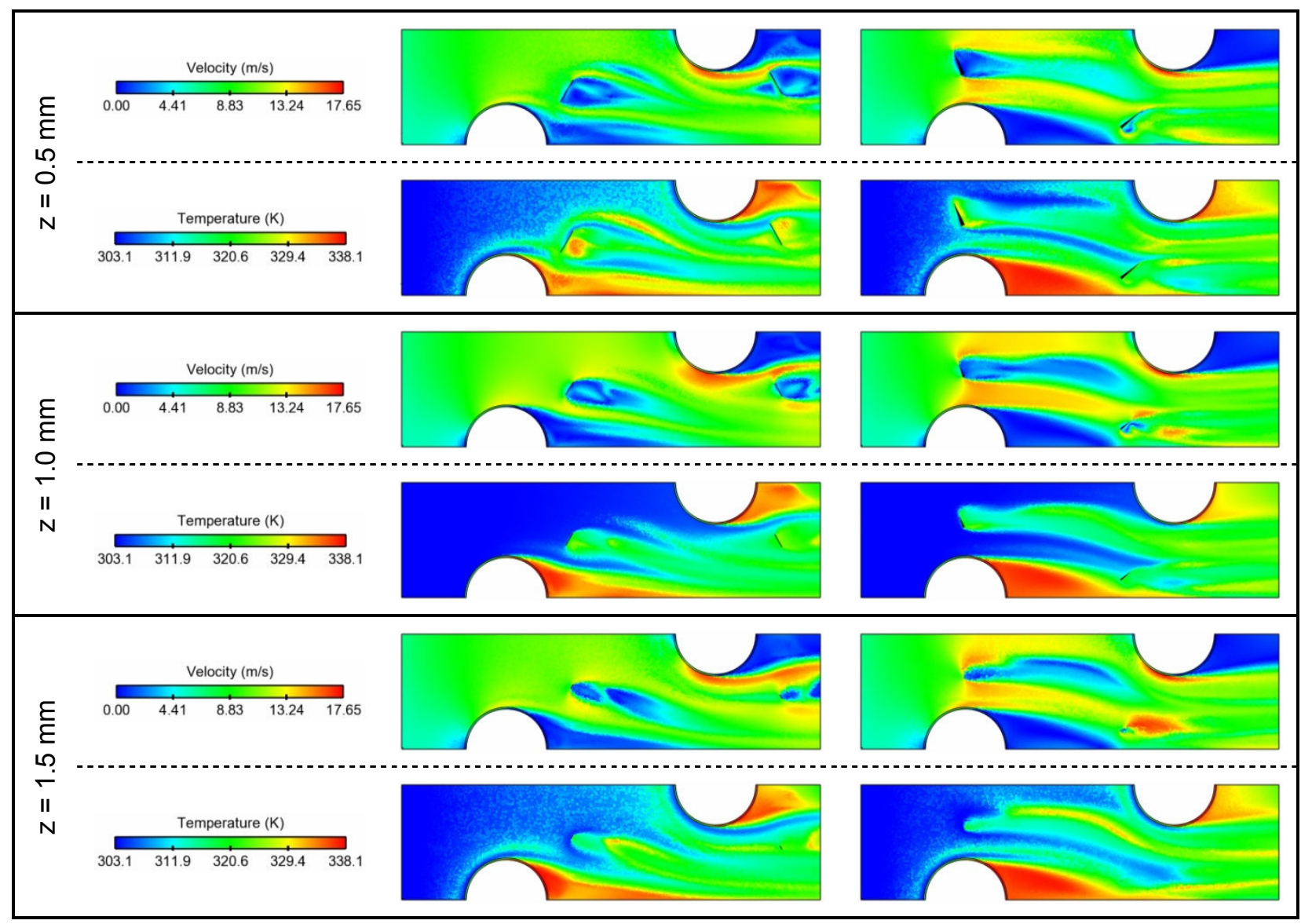

(a) Lemouedda et al. (2010).

(b) j present work (DO).

Figure 106 - Velocity and temperature field planes at axis-z, according to Figure 85. 


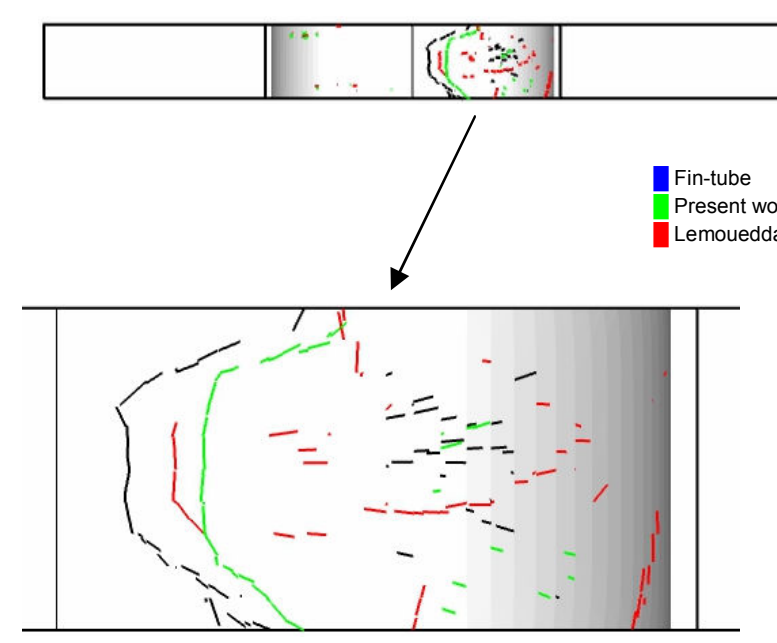

(a)

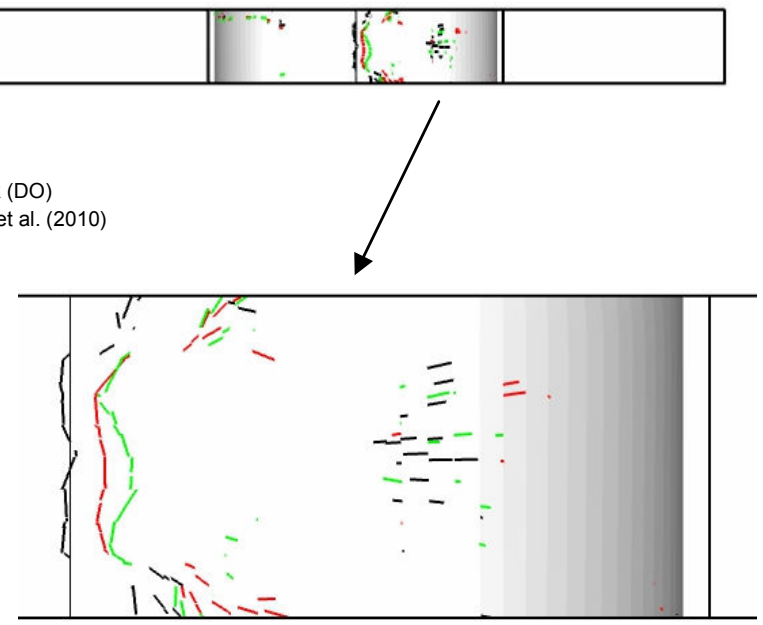

(b)

Figure 107 - Objective function j. Flow separation profile on (a) tube 1, (b) tube 2.

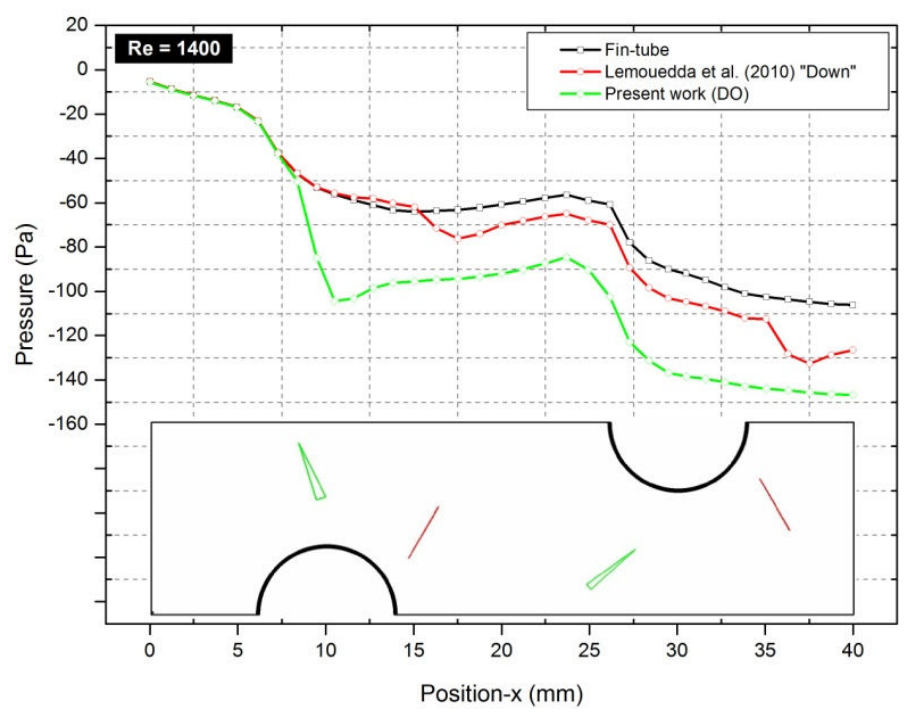

Figure 108 - Pressure profile considering the objective function $\mathrm{j}$.

Optimization procedure for the objective function $\mathrm{j}$ was conducted as a simpleobjective, ie, there is not trade-off between pressure drop and heat transfer rate, since the friction factor was not "controlled". The key features of the longitudinal vortex produced by Lemouedda et al. (2010) arrangement was already presented, Figure 100, which this arrangement was again the best literature work for objective function $\mathrm{j}$. Thus, the pressure drop is not a concern and the Figure 108 shows exactly that the pressure profile by present work (DO) was significantly higher than Lemouedda et al. (2010). VG by the present work (DO) was placed close to minimum 
flow area with very aggressive attack angle $\left(\theta_{1}=287^{\circ}\right)$, causing the high local pressure drop shown in Figure 108. Figure 105 shows the main longitudinal vortex generated by $\mathrm{VG}_{1}$, which was persistent downstream and was not directed onto $\mathrm{VG}_{2}$. About the $\mathrm{VG}_{2}$ arrangement, vortex generator was placed close to wake region of the tube 1 , exactly to avoid the interference between $V G_{2}$ and the main vortex generated by $V_{G_{1}}$; this arrangement was also close to the minimum flow area, which justifies the high local pressure drop, Figure 108. The fluid mixture produced by synergy between $V G_{1}$ and $V G_{2}$ (by present work), is shown in Figure 106. Moreover, although the $V G_{2}$ was placed far from tube 2, the better fluid mixture in the wake region behind of the tube 2 for the present work (DO) was observed, which was due to longitudinal vortex generated by $V_{G_{1}}$. These behaviors clearly explain the persistent and continue higher enhancement heat transfer after the $V G_{1}$ by the present work than Lemouedda et al. (2010), identified in Figure 94. Another interesting result is to regard the separation delay presented in Figure 107. Separation delay produced by the present work (DO) was more pronounced than Lemouedda et al. (2010), both faced to Fin-tube. On the tube 1, Figure 106 visually induces that the VG arrangement from Lemouedda et al. (2010) impacts in the wake region more than in the present work (DO). However, the flow acceleration is the core of this analyze. In fact, a qualitative evaluation through velocity color range, Figure 106, shows that the flow acceleration by the present work was higher than Lemouedda et al. (2010) due to $\mathrm{VG}_{1}$ location. On the tube 1 , slightly separation delay was more pronounced by present work (DO) due to main longitudinal vortex from $\mathrm{VG}_{1}$, which was directed close to tube 2. Exactly as made for $\operatorname{Re}=250$, the concern about the pressure drop was differently weighted for each objective function, ie, pressure drop is more significant for $\mathrm{JF}$ than $\mathrm{JF}^{\frac{1}{3}}$. Moreover, pressure drop was only a consequence for the objective function $\mathrm{j}$. Thus, the pressure in the outlet domain evidences this assessment. In fact, the pressure difference between present work (DO) and Fintube was higher for $\mathrm{j}$ than $\mathrm{JF}^{\frac{1}{3}}$ (Figure 108 and Figure 104, respectively), and was higher for $\mathrm{JF}^{\frac{1}{3}}$ than JF (Figure 104 and Figure 99, respectively). Looking for this three objective functions, the assessment made for $\mathrm{Re}=250$, correlating the rolling angle to pressure drop, was not conclusive for $\operatorname{Re}=1400$, according to Table 8. However, evaluating the rolling angle value for objective function $f(\operatorname{Re}=1400)$, this seems to 
lead to the same conclusions, ie, there is a great relation between rolling angle and global pressure drop. Thus, to finish this flow pattern analysis step, it was conducted an evaluation of the key features related to objective function $\mathrm{f}$, Figure 109 to Figure 111, according to Figure 86. Insofar as possible, was faced the main conclusions between $\operatorname{Re}=250$ and $\operatorname{Re}=1400$.

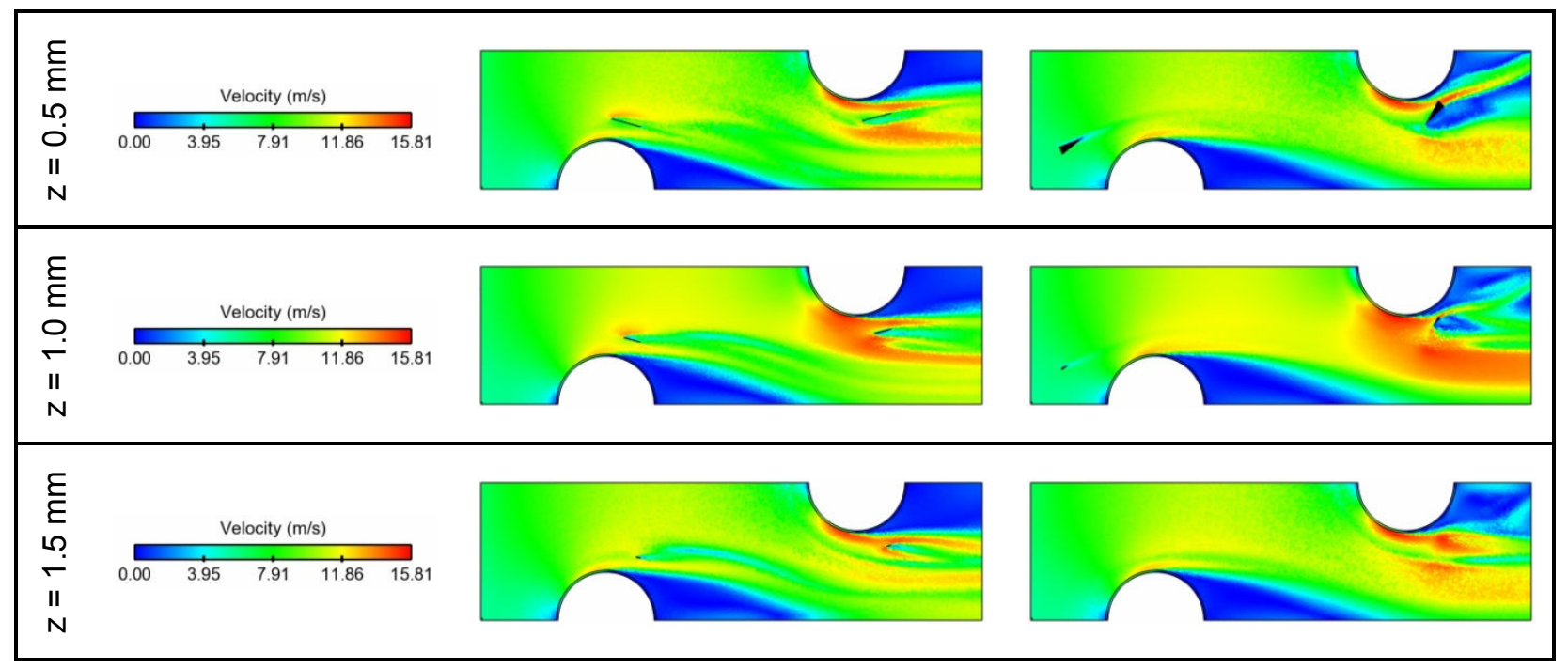

(a) Torii et al. (2010).

(b) f present work (DO).

Figure 109 - Velocity and temperature field planes at axis-z, according to Figure 86.

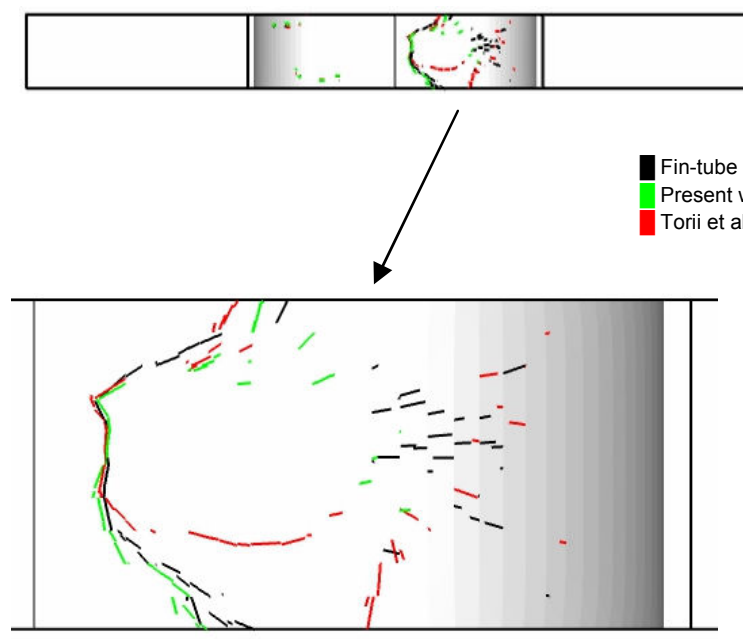

(a)

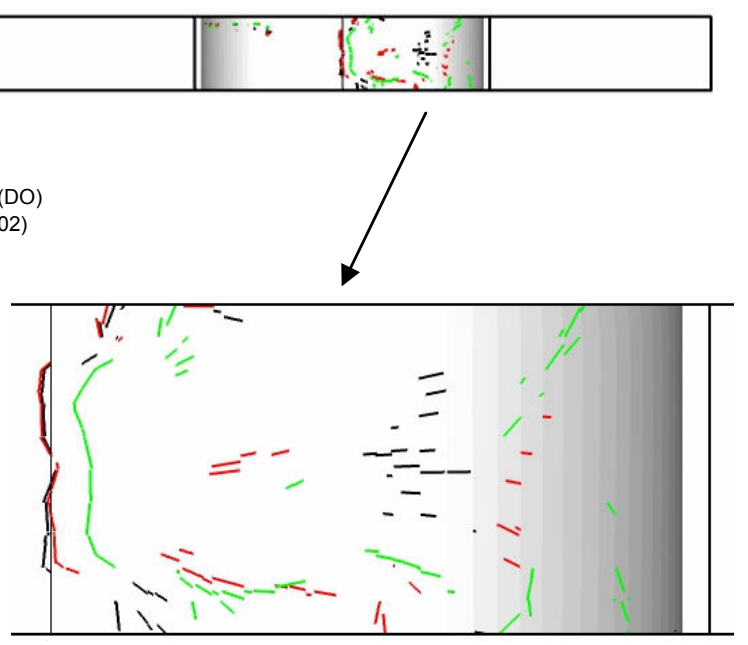

(b)

Figure 110 - Objective function f. Flow separation profile on (a) tube 1, (b) tube 2. 


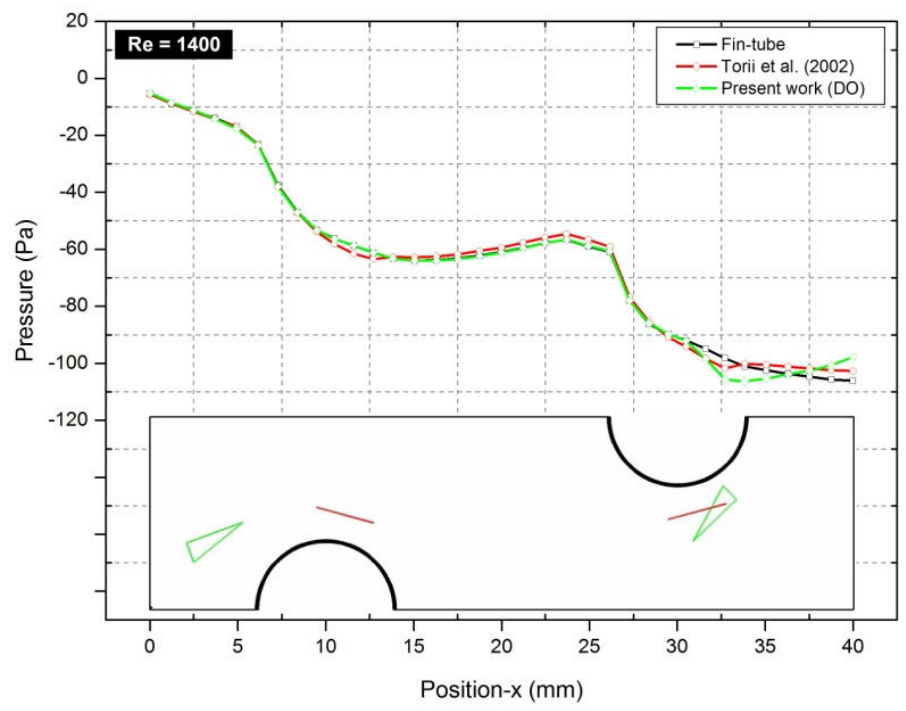

Figure 111 - Pressure profile considering the objective function $\mathrm{f}$.

Figure 109 shows the pressure profile at axis $z$ for the present work (DO) and the best literature work, according to Figure 86. Pressure profile by Torii et al. (2002) configuration was symmetric and shows the called nozzle between VGs and tubes, causing the flow acceleration especially at $z=0.5 \mathrm{~mm}$. However, as perceived for $\operatorname{Re}=250$, there is a net effect of the vortex generator when it is inserted into the flow, ie, the VG naturally increase the pressure drop, however the flow separation delay could decrease this global penalty. For the first tube, Figure 110 shows that the separation delay was achieved for configuration by Torii et al. (2002) and almost nothing to configuration by the present work (DO). For the second tube, separation delay was very soft for Torii et al. (2002) and very significant for the present work (DO), according to flow acceleration shown in Figure $109 \mathrm{~b}$, especially at $z=0.5 \mathrm{~mm}$ and $z=1.0 \mathrm{~mm}$. Figure 111 shows the pressure profile indicating that the pressure profile in some regions from Torii et al. (2002) was lower than present work (DO) and also to fin-tube, further pronounced in region within the first vortex generator up to second tube. Evaluating the impact of the $\mathrm{VG}_{1}$ on the pressure profile, according to VG arrangement by the present work (DO), Figure 111 shows that the $\mathrm{VG}_{1}$ was almost undetectable by the flow, since the profiles (present work and Fin-tube) were coincident in this region. Figure 112 shows a highlight in these regions at axis- $z$, comparing the velocity vector from Fin-tube and present work (DO). 


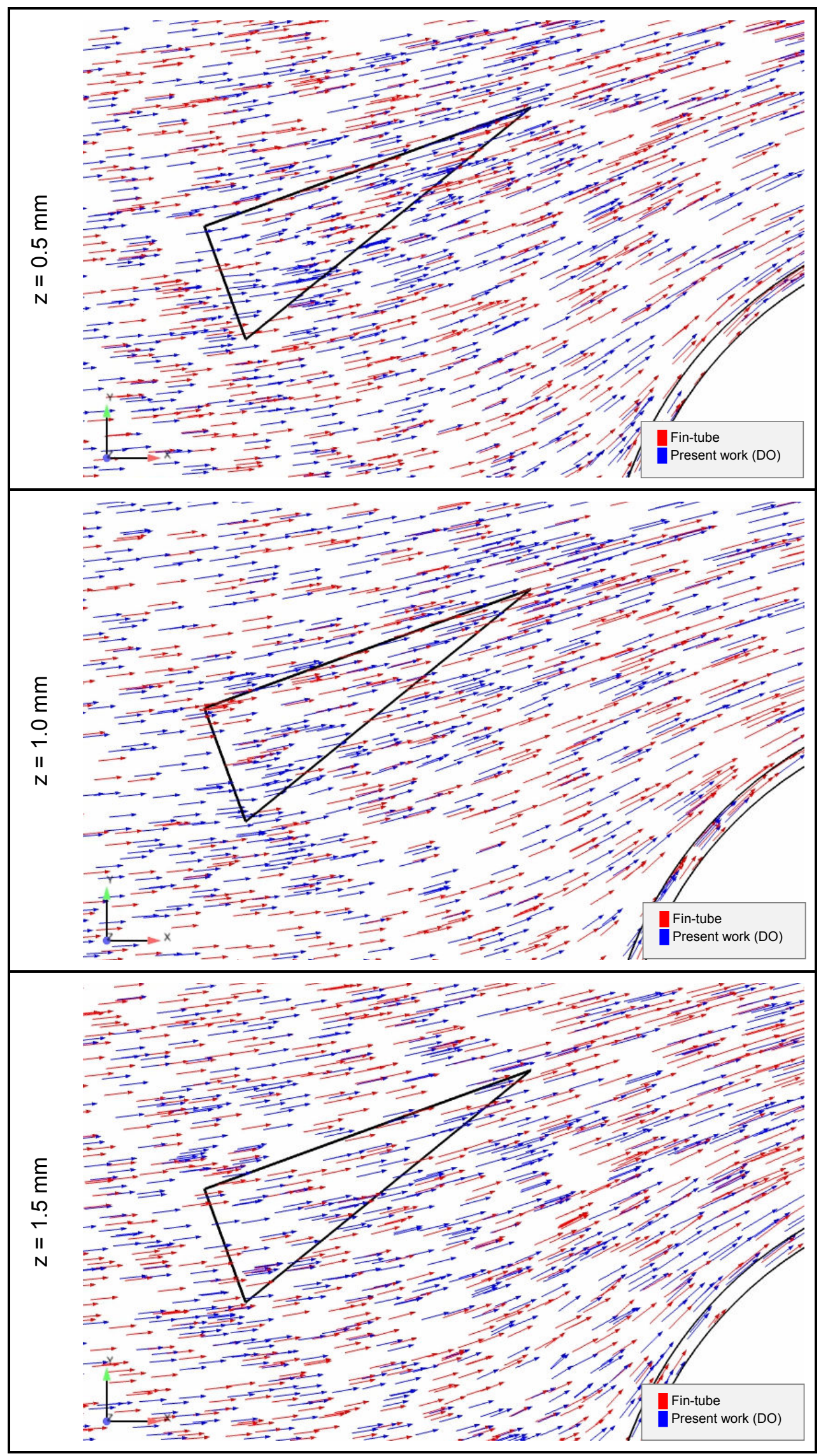

Figure 112 - Highlight at axis-z around the $\mathrm{VG}_{1}$ according to Figure $109 \mathrm{~b}$. 
Figure 112 shows the velocity vector from Fin-tube (without vortex generator) - red vector, and for the present work (DO) - blue vector. The presence of the $\mathrm{VG}_{1}$ in the flow was almost undetectable since the vector directions are very similar at all plane$z$, ie, the attack angle of the $V G_{1}$ was similar to angle of the streamlines from Fin-tube configuration. This assessment evidences the results shown by Figure 111 and Figure 110a. In this case, exactly as concluded for $R e=250$ for objective function $f$, the $V G_{1}$ could be suppressed of the analysis. Therefore, an additional analysis was conducted eliminating the $V G_{1}$ and remaining the $V G_{2}$. The results indicated $a$ difference of $0.21 \%$, ie, the results are numerically equal. Thus, for the objective function $f$, the $V G_{1}$ is irrelevant, according to was already concluded for $\operatorname{Re}=250$. Also, considering the present work (DO) configuration, another analysis was made by positioning the $\mathrm{VG}_{1}$ symmetrically to $\mathrm{VG}_{2}$. Again, as made for $\operatorname{Re}=250$, the rationale for this is to evaluate the local effect on the second tube, Figure 111, on the first tube. The result was $8.24 \%$ higher than previous optimal configuration. These corroborate with the last assessment which state that the $V_{G_{1}}$ is important to enhancement heat transfer and, consequently, increase the global pressure drop. The $\mathrm{VG}_{1}$ arrangement, Figure 112, was found by the optimizer because VG has no thickness. Thus, the VG most appropriated position for this objective function would be in the wake region, according to $\mathrm{VG}_{1}$ position for $\mathrm{Re}=250$.

A last question raised is to compare both optimal VG arrangement for $\operatorname{Re}=250$ and $\mathrm{Re}=1400$. In fact, for both the $\mathrm{VG}_{1}$ was considered not essential to meet the objective function $f$. However, the optimization procedure conducted the optimal arrangement for different position, ie, for $\mathrm{Re}=250$ the $\mathrm{VG}_{1}$ was placed behind of the first tube in the wake region, whereas for $R e=1400$ the $V G_{1}$ was placed in front of first tube. Thus, an additional analysis was made considering the $\mathrm{VG}_{1}$ arrangement according to $\operatorname{Re}=250$ (in the wake region) and remaining $V_{G_{2}}$ according to $\operatorname{Re}=1400$. The difference was $0.1 \%$ between this last arrangement and the original optimal arrangement for $\operatorname{Re}=1400$, ie, the results are numerically equal. Therefore, these locations for $V G_{1}$ are neutral when the objective function $f$ is the main goal, which $V_{G_{1}}$ for both $\operatorname{Re}=250$ and $\operatorname{Re}=1400$ could be suppressed, and the optimal place depends on the convergence for each optimization procedure, and also because the $\mathrm{VG}_{1}$ has not thickness. 
Overall, Figure 113 and Figure 114 present a qualitative comparison for each objective function at $\operatorname{Re}=250$ and $\operatorname{Re}=1400$, showing a top and perspective view, according to VG arrangements by the present work (DO), which was the best results among RSM approach and literature researches. These pictures only summarize the arrangements outlined and reported in Table 8. Strengthening the previous conclusions is evident that there is not symmetry between $V G_{1}$ and $V G_{2}$ for both Reynolds number, Figure 113. Moreover, it was observed that it does not exist one unique arrangement to meet simultaneously all objective functions evaluated herein, Figure 114, for an unchanged Reynolds number. Evaluating all VG arrangements for DO approach, some trends could be indicated, such as: $V G_{1}$ was rather placed around the minimum flow area (close to tube 1), and $V G_{2}$ was placed slightly after the minimum flow area (close to tube 2). These trends were observed for both Reynolds number, according to Figure 114. Obviously, for each objective function and Reynolds number, an optimal set of the input variable was defined through optimization procedure, in accordance with assessments made in the last sections. 

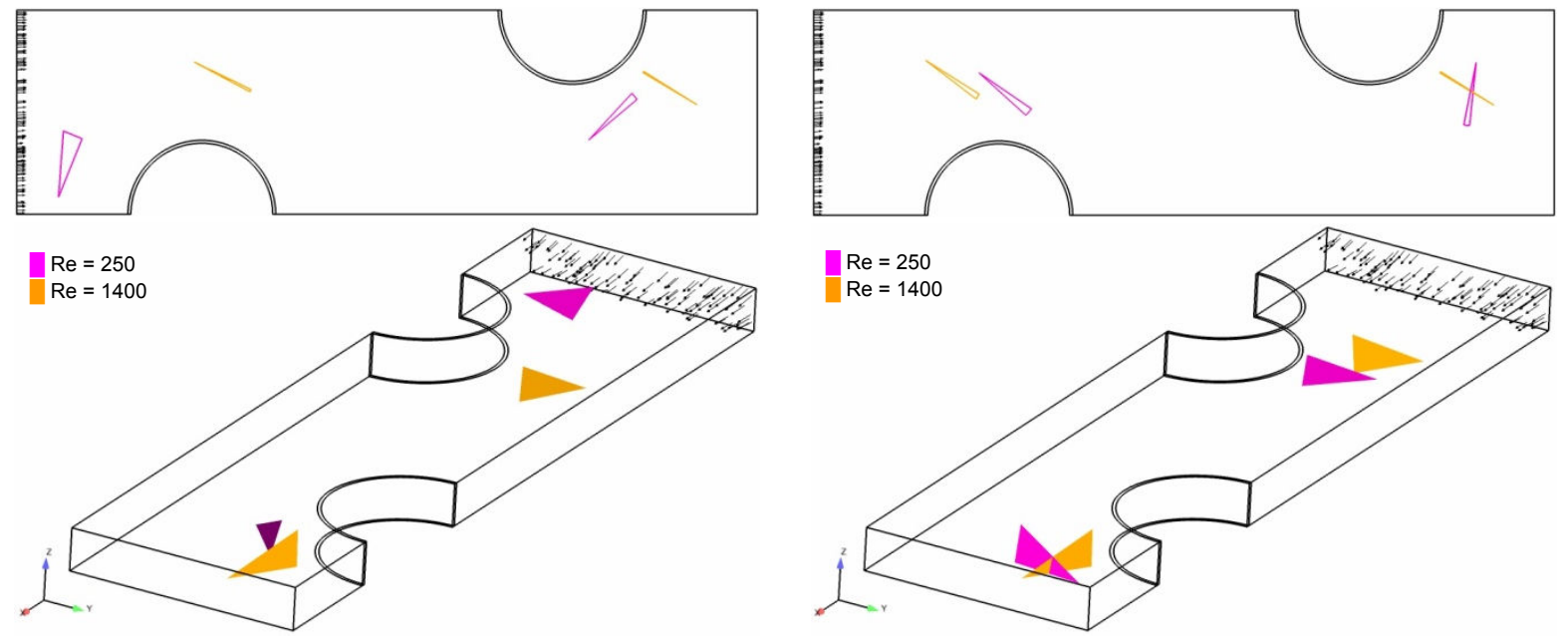

(a) JF

(b) $\mathrm{JF}^{\frac{1}{3}}$
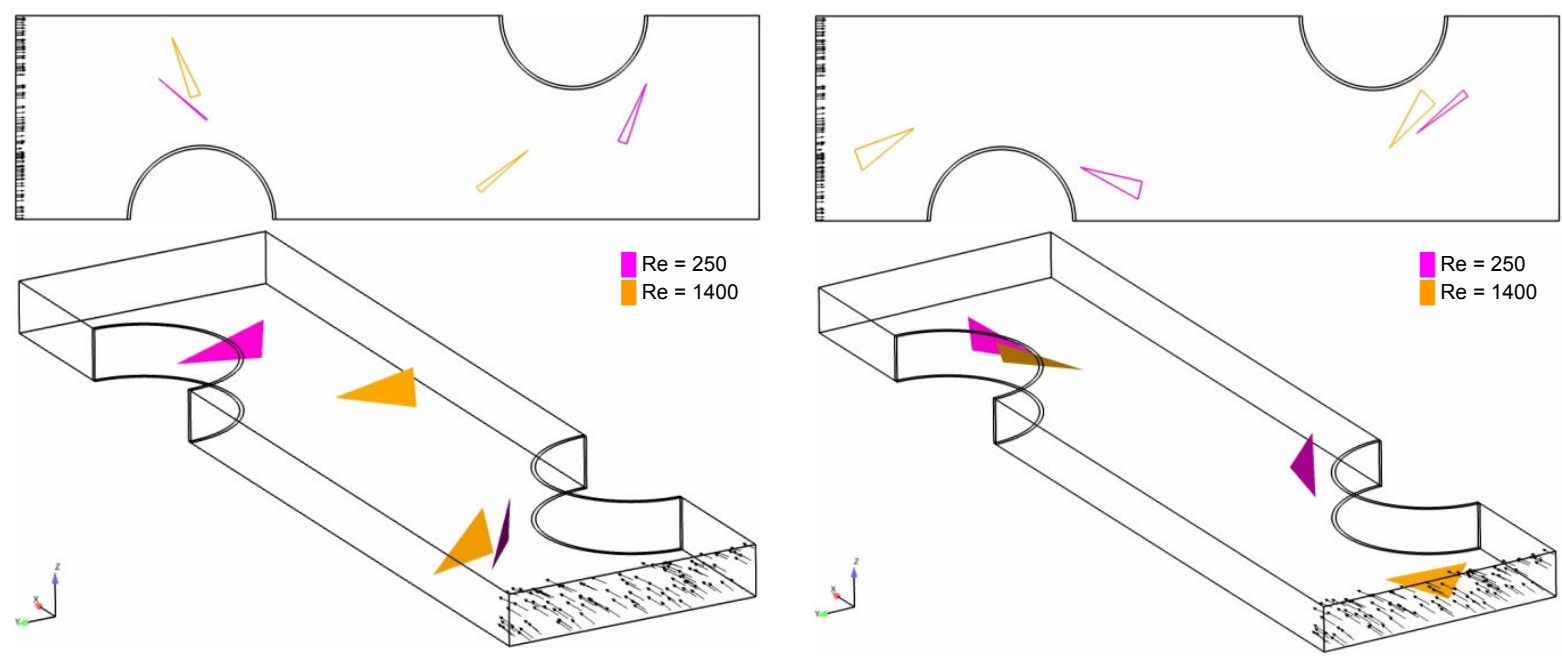

(c) Colburn factor, j

(d) Friction factor, $f$

Figure 113 - Comparison between vortex generator arrangements for different Reynolds number found by optimizer for each objective functions.

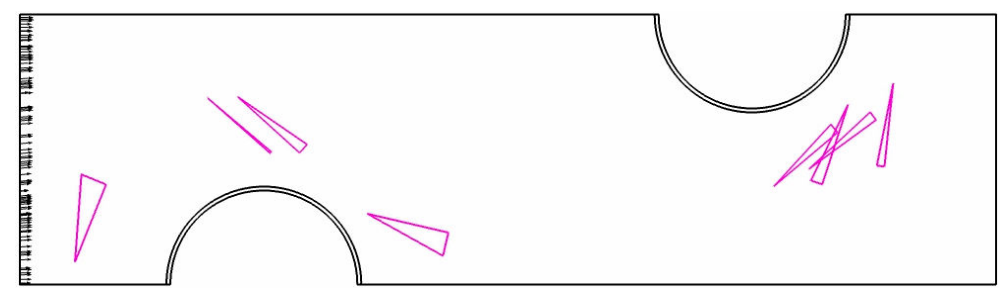

(a) $\operatorname{Re}=250$

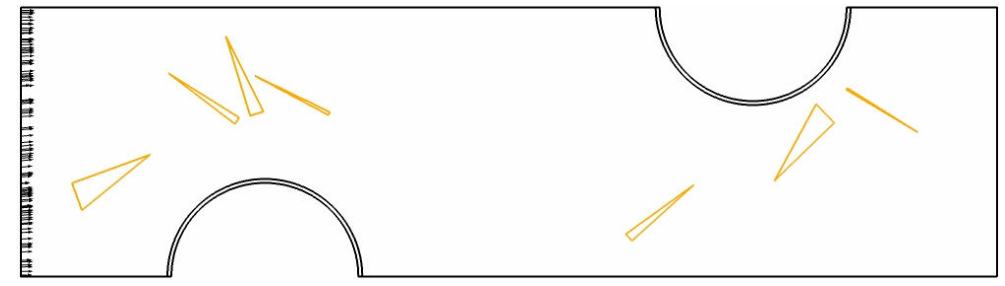

(b) $\operatorname{Re}=1400$

Figure 114 - Vortex generator arrangements by present work (DO) for all objective functions. 
Concluding this chapter and only as an additional information, Figure 115 and Figure 116 present a global result comparison for each objective functions at different Reynolds number for the present work (DO), considering the following approach:

- In Figure 115, each objective function indicated with superscript (a), at $\operatorname{Re}=250$, was calculated under optimal VG arrangement from $\operatorname{Re}=1400$. For example, $\mathrm{JF}^{(\mathrm{a})}$ was calculated considering the $\mathrm{VG}$ arrangement shown in row 16 in Table 8 (corresponding to JF at $\operatorname{Re}=1400$ ).

- Otherwise, in Figure 116, each objective function indicated with superscript (b), at $\operatorname{Re}=1400$, was calculated under optimal VG arrangement from $\mathrm{Re}=250$. For example, $\mathrm{j}^{(\mathrm{b})}$ was calculated considering the vortex generator arrangement shown in row 12 in Table 8 (corresponding to $\mathrm{j}$ at $\mathrm{Re}=250$ ).

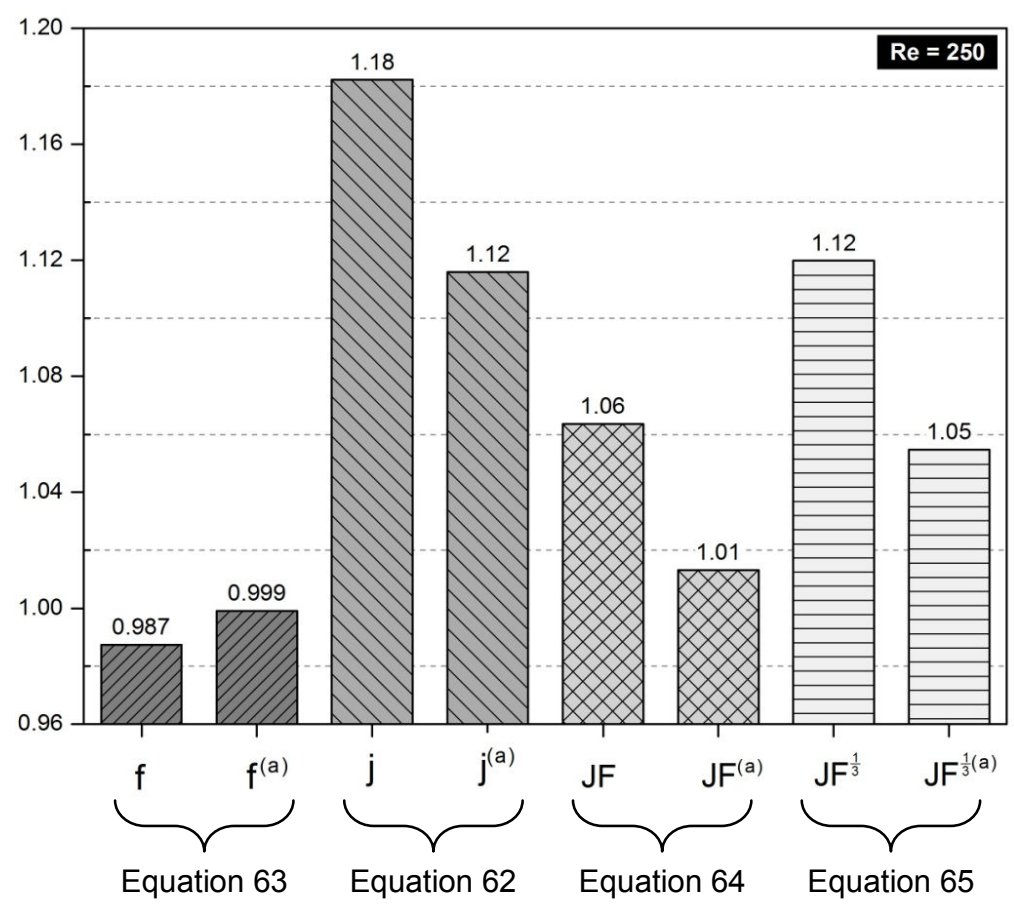

Figure 115 - Comparison among global results for each objective function at $\operatorname{Re}=250$. 


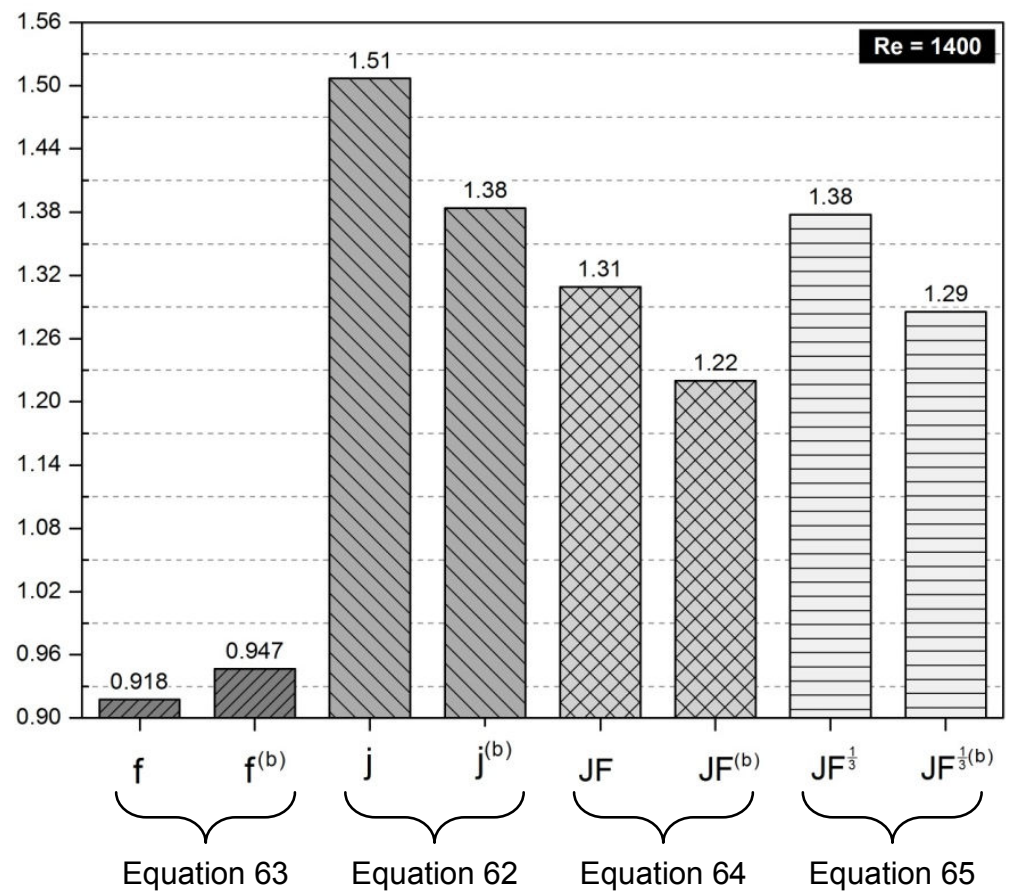

Figure 116 - Comparison among global results for each objective function at $\operatorname{Re}=1400$.

For both Figure 115 and Figure 116, the original optimal VG arrangements were better than "superscript" arrangements. Therefore, these comparisons only confirm that the optimal VG arrangement found for a specific objective function at an unchanged Reynolds number is not necessarily the best choice for another Reynolds number, considering the same objective function. This shows that the knowledge of the application is fundamental at the beginning of a heat exchanger project which optimization tools is applied. Thus, define the worst case for each application is necessary; however, it is suitable to evaluate this at different project points, since the heat exchanger is under several application conditions, as for example a radiator for automotive applications. 


\section{Conclusions}

The present thesis has dealt with heat transfer enhancement in compact heat exchanger with two rows of tubes in a staggered arrangement by means of delta-winglet type longitudinal vortex generators. A compact heat exchanger typically applied on automotive industry was chosen under certain conditions, which correspond to $\operatorname{Re}=250$ and $\operatorname{Re}=1400$, based on fin pitch. Some parameters of the delta-winglet vortex generator, such as attack angle and position in direction $\mathrm{x}$ and $\mathrm{y}$, were well studied by current researches. Moreover, the VG arrangement for the first tube was symmetrically positioned at second tube. Thus, current researches usually studies three independent variables considering a few combinations of these variables, where the "optimal" arrangement was normally chosen among them. In the present work, a thorough single-objective optimization was conducted, considering four independent input variables for each vortex generator, amounting eight parameters. Among these input variables, rolling angle $(\varphi)$ was firstly proposed in this present work. Two main factors were evaluated, Colburn factor (j) and Friction factor (f), where, through them, four objective functions were defined: JF, $\mathrm{JF}^{\frac{1}{3}}, \mathrm{j}$ and $\mathrm{f}$. CFD methodology was applied to solve the governing equations in $3 \mathrm{D}$ physical space through a commercial software, which was based on Finite Volume Methodology. Two optimization procedures were adopted: Response Surface Methodology (RSM) and Direct Optimization (DO). Optimal arrangement for each objective function at a certain Reynolds number was compared to the main results from the open literature surveys. The conclusions drawn from the present research were:

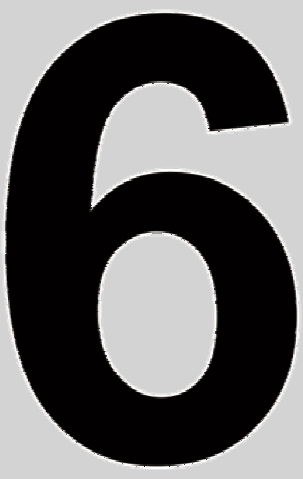


1. Numerical methodology applied here was enough to identify the major phenomena related to augmentation heat transfer in compact heat exchanger, according to guide-line from literature researches;

2. Conclusions by Factor Analysis Methodology corroborates very well with key features from flow pattern study;

3. Direct Optimization (DO) reported better results than Response Surface Methodology (RSM), for all objective functions;

4. Error introduced by RSM, in optimization workflow, should be decreased even though the recommendations by Forrester et al. (2008) were met, according to result in Appendix B;

5. Enhance the heat transfer at low velocity $(\operatorname{Re}=250)$ was much more difficult than to high velocity $(\operatorname{Re}=1400)$. This conclusion was already reported by current researches;

6. Both optimization procedures, DO and RSM, were able to find VG arrangement, for each objective function, better than results from current literature researches;

7. The better results, for each objective functions, were achieved when $V G_{1}$ was not symmetrical to $V_{G_{2}}$. Therefore, the input variables for each $V G$ should be evaluated independently;

8. $V G_{1}$ position directly affects the $V G_{2}$ position during the convergence step of the optimization procedure for all objective functions and both Reynolds number;

9. There are important interactions among input variables, which impacted at different level on Colburn and Friction factors. These interactions were different for each Reynolds number;

10. For each Reynolds number and each objective function, there is an optimal VG arrangement, where is not necessarily the better choice for another objective function; 
11. Rolling angle $(\varphi)$, proposed herein, showed significant impact on both Colburn and Friction factors, beyond important interaction with another input variable, such as: $x_{1} \varphi_{1}$ on $f(R e=250)$ and $\varphi_{2} \theta_{2}$ on $j(R e=1400)$;

12. For objective function $\mathrm{f}, \mathrm{VG}_{1}$ did not contribute to mitigate the global pressure drop for both Reynolds number $(\operatorname{Re}=250$ and $\operatorname{Re}=1400)$, whereas $V G_{2}$ was very important to flow separation delay on tube 2 ;

13. In general, for objective function $\mathrm{JF}, \mathrm{JF}^{\frac{1}{3}}$ and $\mathrm{j}$, the longitudinal vortices from $V G_{1}$ was not directed onto $V G_{2}$, even when the pressure drop was not a concern (objective function j);

14. The main goal of the $V G_{1}$ was to increase the heat transfer rate, whereas the mitigation of the pressure loss was a second goal. For $\mathrm{VG}_{2}$, the main goals were both increase the heat transfer rate and decrease the local pressure behind the tube 2. The exception of this assessment was verified for $\operatorname{Re}=1400$ to objective function $\mathrm{j}$. In this case, the root cause is based on conclusion at previous conclusion 13;

15. Except for objective function JF $(R e=250)$ and objective function $f(R e=250$ and $R e=1400), V G_{1}$ was placed close to minimum flow area around the tube 1. In this region is verified the maximum average velocity, which generated stronger longitudinal vortex with an appropriate attack and rolling angles;

16. The net effect of the VG immersed into the flow on global pressure drop was different for each tube. On tube 1, the effect was harmful since the advantage of the separation delay was lower than pressure drop, due to overall impact of the longitudinal vortex up to outlet domain. On tube 2, this mind-set was opposite since the distance between $V G_{2}$ and outlet domain is small. The evidences of this assessment were the optimal VG arrangement found to objective function $\mathrm{f}$, for both Reynolds number;

17.Evaluating only researches from open literature, common-flow-up arrangement was the best choice when global pressure drop is a concern on objective function (for example, JF), and common-flow-down was indicated when higher heat transfer rate is desired $\left(\mathrm{j}\right.$ and $\left.\mathrm{JF}^{\frac{1}{3}}\right)$; 


\subsection{Scope for Future Work}

Based on this study, a great deal of work could be conducted for heat transfer enhancement, using longitudinal VG in the future:

a) In the present work, numerical methodology considered constant temperature boundary conditions along the walls. The model presented an idealized situation. A more accurate model could be considered, regarding to finite thickness of the plates of the CHE and the VG, which ought to be solved the conjugate heat transfer problem. The solution of the conjugate heat transfer problem can be expected to yield predictions that are more exact;

b) Evaluate the same approach described here on inline arrangement tubes, under the same boundary conditions.

c) Extend the present work for different geometries of the VG, considering the possibility of different type for each tube.

d) Consider other parameters in optimization procedure, such as:

i. Longitudinal, transverse and fin pitch;

ii. Diameter of tube;

iii. Aspect ratio of the vortex generator;

iv. Reynolds number.

e) Analyzing the optimization procedure for heat exchanger with multiple row tubes for both inline and staggered arrangements.

f) Appling optimization tools to other techniques of enhancement heat transfer.

g) Evaluating other optimization algorithms, such as those based on gradient and non-gradient.

h) Applying all outlined recommendations considering oval tube. 
ABDEL-MAGID, Y.L. AND DAWOUD, M.M. Tuning of AGC of interconnected reheat thermal systems with genetic algorithms, Proc. IEEE Int. Conf. Syst. Man Cybernetics, Vol. 3, pp. 2622-2627, 1995.

AHMEDA, H.E., MOHAMMEDB, H.A., YUSOFF, M.Z. An overview on heat transfer augmentation using vortex generators and nanofluids: Approaches and applications Renewable and Sustainable Energy Reviews 16, 5951-5993, 2012.

ALLISON, C.B., DALLY. B.B. Effect of a delta-winglet vortex pair on the performance of a tube-fin heat exchanger. International Journal Heat Mass Transfer; 50(25-26):506572, 2007.

ALLRED, L.G. AND KELLY, G.E. Modified genetic algorithm for extracting thermal profiles from infrared image data, Proc SPIE - Int. Soc. Optical Eng. Neural Stochastic Methods Image and Signal Processing, Vol. 1766, pp. 77-81, 1992.

AMIRI, A. and VAFAI K. Analysis of dispersion effects and nonthermal equilibrium, non-Darcian, variable porosity incompressible flow through porous media. International Journal of Heat and Mass Transfer, vol. 37, no. 6, pp. 939954, 1994.

ANDROULAKIS, I.P. AND VENKATASUBRAMANIAN, V. Genetic algorithmic framework for process design and optimization, Computational. Chem. Eng., 15(4), 217-228, 1991.

ANGELINE, P.J., SAUNDER, G.M., AND POLLACK, J.B. Complete induction of recurrent neural networks, Proc. Third Annu. Conf. Evolutionary Programming, (Eds.) A.V. Sebald, L.J. Fogel, World Scientific, Singapore, pp. 1-8, 1994.

ARMAN, B. and RABAS, T. J. Disruption share effects on the performance of enhanced tubes with the separation and reattachment mechanism. In Proceedings of the 28th National Heat Transfer Conference and Exhibition, vol. 202, pp. 67-75, 1992.

BAUM, E. B. What size net gives valid generalization? Neural Computation, MIT Press, 1989.

BARTH, T. J. AND JESPERSEN, D. The design and application of upwind schemes on unstructured meshes. Technical Report AIAA-89-0366. AIAA 27th Aerospace Sciences Meeting, Reno, Nevada, 1989. 
BERGLES, A. E. Handbook of Heat Transfer, McGraw-Hill, New York, NY, USA, 3rd edition, 1998.

BERGLES, A. E. The implications and challenges of enhanced heat transfer for the chemical process industries. Chemical Engineering Research and Design, vol. 79, no. 4, pp. 437-444, 2001.

BHUTTA, M. M. A., HAYAT, N., BASHIR, M. H., KHAN, A. R., AHMAD, K. N., KHAN, S. CFD applications in various heat exchangers design: A review. Applied Thermal Engineering, 32, pp.1-12, 2012.

BITTANTI, S. and PIRODDI, L. Nonlinear identification and control of a heat exchanger: A neural network approach, J.Franklin Inst.334B(1), 135-153, 1997.

BONNET, A. Artificial Intelligence, Promise and Performance, Prentice-Hall, Englewood Cliffs, NJ, 1985.

BUHMANN, M. D. Radial Basis Functions: Theory and Implementations, Cambridge University Press, 2003.

BULSARI, A.B. AND SAXIN, H. Classification of blast furnace probe temperatures using neural networks, Steel Res., 66, 231-236, 1995.

CASTROGIOVANNI, A. AND SFORZA, P.M. Scaling of high heat flux flow boiling flow systems, Proc. ASME Heat Transfer Division, Int. Mech. Eng. Congress. Exposition, Vol. 334, No. 3, pp. 39-46, 1996.

CASTROGIOVANNI, A. AND SFORZA, P.M. A genetic algorithm model for high heat flux flow boiling, Exp. Thermal Fluid Sci., 15(3), 193, 1997.

CHARNIAK, E. AND MCDERMOTT, D. Introduction to Artificial Intelligence, AddisonWesley, Reading, MA, 1985.

CHIPPERFIELD, A. Introduction to genetic algorithms, in Genetic Algorithms in Engineering Systems, (Eds.) A.M.S. Zalzala, P.J. Fleming, Institute of Electrical and Electronics Engineers, New York, 1997.

DAVALOS, R.V. AND RUBINSKY, B. An evolutionary-genetic approach to heat transfer analysis, ASME J. Heat Transfer, 118(3), 528, 1996.

DHAR, A., CLARIDGE, D.E., AND RUCH, D. Artificial neural network with wavelet basis functions to predict hourly heating and cooling energy use in commercial buildings with temperature as the only weather variable, Proc. ASME-JSMEJSES Int. Solar Energy Conf., Vol. 1, pp. 145-156, 1995.

DIAZ, G., YANES, J., SEN, M., YANG, K.T., AND MCCLAIN, R.L. Analysis of data from single-row heat exchanger experiments using an artificial neural network, FED. Proc. ASME Int.Mech.Eng.Congress. Exposition, Vol.242, pp.45-52, 1996.

DIAZ, G., SEN, M., YANG, K.T., AND MCCLAIN, R.L. Neurocontrol of Heat Exchangers, Proc. 4th Int. Meet. Mech. Eng., Mexico, pp. 123-128, 1998. 
DIAZ, G., SEN, M., YANG, K.T., AND McCLAIN, R.L. Int. J. HVAC\&R Res., 5(3), 195-208, 1999.

DING, Y., ALIAS, H., WEN, D., and WILLIAMS, R. A. Heat transfer of aqueous suspensions of carbon nanotubes (CNT nanofluids). International Journal of Heat and Mass Transfer, vol. 49, no. 1-2, pp. 240-250, 2006.

DOUNIS, A.I., SANTAMOURIS, M.J., AND LEFAS, C.C. Implementation of artificial intelligence techniques in thermal comfort control for passive solar buildings, Energy Conversion. Manage., 33(3), 175-182, 1992.

DRAGO, G.P. and RIDELLA, S. Statistically controlled activation weight initialization, IEEE Trans. Neural Networks, 3(4), 627-631, 1992.

EDWARDS, F. J. and ALKER, C. J. R., 1974. The improvement of forced convection surface heat transfer using surface protrusions in the form of (a) cubes and (b) vortex generators, Heat Transfer 1974, Proc Fifth International Heat Transfer

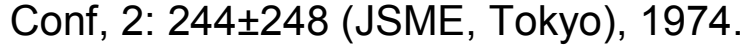

EECKMAN, F.H. Analysis and Modeling of Neural Systems. Kluwer Academic, Boston, 1992.

EIAMSA-ARD, S., THIANPONG, C., EIAMSA-ARD, P. Turbulent heat transfer enhancement by counter/co-swirling flow in a tube fitted with twin twisted tapes. Experimental Thermal and Fluid Science 34, 53-62, 2010.

FABBRI, G. A genetic algorithm for fin profile optimization, Int. J. Heat Mass Transfer, 40(9), 2165, 1997.

FERRANO, F.J. AND WONG, K.-F.V. Prediction of thermal storage loads using a neural networks, ASHRAE Trans., Pt. 2, 723-726, 1990.

FIEBIG, M., VALENCIA, A., MITRA, N.K. Wing-type vortex generators for fin-andtube heat exchangers, Exp. Thermal Fluid Sci. 7, 287-295, 1993.

FIEBIG, M. Vortex generator for compact heat exchangers. Journal Enhanced Heat Transfer, 2, 43-62, 1995.

FIEBIG, M. Vortices, Generators and Heat Transfer. Trans. IchemE, Vol.76, 1998.

FLOOD, I. AND KARTAM, N. Neural networks in civil engineering. I. Principles and understanding, ASCE J. Computational. Civil Eng., 8(2), 131-148, 1994.

FLUENT INC., Ansys Fluent: Theory Guide, 2010.

FOWLER, M.M., KLETT, D.E., MORENO, J.B., AND HEERMANN, P.D. Using artificial neural networks to predict the performance of a liquid sodium reflux pool boiler solar receiver, Proc. Int. Solar Energy Conf., pp. 93-104, 1997.

FORRESTER, A. I. J., SÓBESTER, A. AND KEANE, A. J. Engineering Design via Surrogate Modelling: A Practical Guide. A John Wiley and Sons, Ltd., Publication, 2008. 
FORTUNA, L., MUSCATO, G., NUNNARI, G., AND PAPALEO, R. Neural network approach to control the temperature on rapid thermal processing. Proc. 8th Mediterranean Electrotech. Conf. MELECON Ind. Appl. Power Systems, Computational. Sci. Telecommun., Part 2, Vol. 2, pp. 649-652, 1996.

GAGARIN, N., FLOOD, I., AND ALBRECHT, P. Computing track attributes with artificial neural networks, J.Computational. Civil Eng.,8(2), 179-200, 1994.

GOBOVIC, D. AND ZAGHLOUL, M.E. Design of locally connected CMOS neural cells to solve the steady-state heat flow problem, Proc. 36th Midwest Symp. Circuits Systems, Vol. 1, pp. 755-757, 1993.

GOLDBERG, D.E. Genetic Algorithms in Search, Optimization and Machine Learning, Addison-Wesley, Reading, MA, 1989.

GONG, J., MIN, C., QI, C., WANG, E., TIAN, L. Numerical simulation of flow and heat transfer characteristics in wavy fin-and-tube heat exchanger with combined longitudinal vortex generators. International Communications in Heat and Mass Transfer 43, 53-56, 2013.

HAGGE, J. K. and JUNKHAN, G. H. Experimental study of a method of mechanical augmentation of convective heat transfer in air. Tech. Rep. HTL3, ISU-ERIAmes-74158, lowa State University, Amsterdam, The Netherlands, 1975.

HAYKIN, S. Neural Networks: A Comprehensive Foundation, Macmillan, NY, 1994.

HE, Y.L., HAN, H., TAO, W.Q., ZHANG, Y.W. Numerical study of heat-transfer enhancement by punched winglet-type vortex generator arrays in fin-and-tube heat exchangers. Int. Journal of Heat and Mass Transfer 55, 5449-5458, 2012.

HE, Y-L, AND ZHANG, Y. Advances and Outlooks of Heat Transfer Enhancement by Longitudinal Vortex Generators. Advances in Heat Transfer, Volume 44, ISSN 0065-2717, 2012.

HECHT-NIELSEN, R. Kolmogorov's mapping neural network existence theorem. IEEE First Int. Conf. Neural Networks, Vol. III, pp. 11-13, 1987.

HERPE, J., BOUGEARD, D., RUSSEIL, S., STANCIU, M. Numerical investigation of local entropy production rate of a finned oval tube with vortex generators. International Journal of Thermal Sciences 48, 922-935, 2009.

HILBERT, R., JANIGA, G., BARON, R., THÉVENIN, D. Multi-objective shape optimization of a heat exchanger using parallel genetic algorithms. International Journal of Heat and Mass Transfer 49, 2567-2577, 2006.

HOERNER, S.F. Fluid-Dynamic Lift. Hoerner Fluid Dynamics, 1985.

HOLLAND, J.H. Adaptation in Natural and Artificial Systems, University of Michigan Press, Ann Arbor, 1975.

HOLLAND, J.H. Genetic algorithms, Sci. Am., 267(1), 66, 1992. 
HU, W-L., SONG, K-W., GUAN, Y., CHANG, L-M., LIU, S., WANG, L-B.. Secondary flow intensity determines Nusselt number on the fin surfaces of circle tube bank fin heat exchanger. Int. Journal of Heat and Mass Transfer 62, 620-631, 2013.

HUISSEUNE, H., T'JOEN, C., DE JAEGER, P., AMEEL, B., DE SCHAMPHELEIRE, S., DE PAEPE, M. Influence of the louver and delta winglet geometry on the thermal hydraulic performance of a compound heat exchanger. International Journal of Heat and Mass Transfer 57, 58-72, 2013.

HUSH, D. R. Classification with neural networks: a performance analysis. In: Proceedings of the IEEE International Conference on Systems Engineering, Dayton, Ohio, pp.277-280, 1989.

HWANG, S.W., KIM, D.H., MIN, J.K., JEONG, J.H. CFD analysis of fin tube heat exchanger with a pair of delta winglet vortex generators. Journal of Mechanical Science and Technology 26 (9), 2949 2958, 2012.

INCROPERA, F. P., DE WITT, A. P. Fundamentals of heat and mass transfer, Fifth Edition. John Wiley \& Sons, Inc, 2002.

INOUE, Y., SUGIURA, S., MURAKAMI, K., and KOTERA, N. Thermal comfort and climate control, ASHRAE J., 37(10), 28-32, 1995.

ITO, K., SHIBA, T., and YOKOYAMA, R. Operational advisor for a heat pump/thermal storage system (development and evaluation of a method based on a layered neural network), Trans. Jpn. Soc. Mech. Eng., C, 61(590), 3909-3915, 1995.

JACOBI, A. M., SHAH, R. K. Heat Transfer Surface Enhancement through the Use of Longitudinal Vortices: A Review of Recent Progress. Exp. Thermal and Fluid, Elsevier Science Inc 655 Avenue of the Americas, NY, pp.295-309, 1995.

JAMBUNATHAN, K., HARTLE, S.L., ASHFORTH-FROST, S., AND FONTAMA, V.N. Evaluating convective heat transfer coefficients using neural networks, Int. J. Heat Mass Transfer, 39(11), 2329-2332, 1996.

JENKINS, W.M. Neural network-based approximations for structural analysis, in Developments in Neural Networks and Evolutionary Computing for Civil and Structural Engineering, (Ed.) B.H.V. Topping, Civil-Computing Press, Edinburgh, U.K., 25-35, 1995.

JOARDAR, A., JACOBI, A.M. Heat transfer enhancement by winglet-type vortex generator arrays in compact plain-fin-and-tube heat exchangers. International Journal of Refrigeration 3, 87- 97, 2008.

JOHNSON, T. R. and JOUBERT, P. N. The influence of vortex generators on the drag and heat transfer from a circular cylinder normal to an airstream. Journal

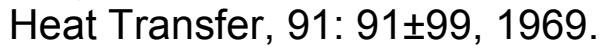

JONES, M.R., TEZUKA, A., AND YAMADA, Y. Thermal tomographic detection of inhomogeneities, ASME J. Heat Transfer, 117(4), 969-975, 1995.

KAASTRA, I., BOYD, M. Designing a neural network for forecasting financial and economic time series. Neurocomputing 10, pp.215-236, 1996. 
KAMARTHI, S., SANVIDO, V., AND KUMARA, R. Neuroform-neural network system for vertical form-work selection, J. Computational, 6(2), 178-199, 1992.

KAMINSKI, W., STRUMILLO, P., AND TOMCZAK, E. Genetic algorithms and artificial neural networks for description of thermal deterioration processes, Drying Technol., 14(9), 2117-2133, 1996.

KARMIN, E.D. Simple procedure for pruning back propagation-trained neural networks, IEEE Trans. Neural Networks, 1(2), 239-242, 1990.

KAWASHIMA, M., DORGAN, C.E., AND MITCHELL, J.W. Hourly thermal load prediction for the next 24 hours by Arima, Ewma, LR, and an artificial neural network, ASHRAE Trans., 1, 186-200, 1995.

KENWRIGHT, D. Automatic Detection of Open and Closed Separation and Attachment Lines”, IEEE Visualization ‘98, pp. 151-158, 1998.

KENWRIGHT, D., HAIMES, R. Vortex Identification - Applications in Aerodynamics, IEEE Visualization ‘97, 1997.

KREITH, F., TIMMERHAUS, K., LIOR, N., SHAW, H., SHAH, R.K., BELL, K. J., DILLER, K. R., VALVANO, J. W. The CRC Handbook of Thermal Engineering. CRC Press LLC, 2000.

KWAK, K.M., TORII, K., NISHINO, K. Simultaneous heat transfer enhancement and pressure loss reduction for finned-tube bundles with the first or two transverse rows of built-in winglets. Exp Thermal Fluid Science; 29(5):625-32, 2005.

KHALED, A.-R. A. and VAFAI K. Flow and heat transfer inside thin films supported by soft seals in the presence of internal and external pressure pulsations. Int. Journal of Heat and Mass Transfer, vol. 45, no. 26, pp. 5107-5115, 2002.

KHALED, A.-R. A. and VAFAI K. Cooling enhancements in thin films supported by flexible complex seals in the presence ultrafine suspensions. Journal of Heat Transfer, vol. 125, no. 5, pp. 916-925, 2003.

KHALED, A.-R. A. The role of expandable thermal systems in improving performance of thermal devices. International Journal of Thermal Sciences, vol. 46, no. 4, pp. 413-418, 2007.

KHALED, A.-R. A. Heat transfer analysis through solar and rooted fins. Journal of Heat Transfer, vol. 130, no. 7, Article ID 074503, 2008.

KIM, S.-E. AND CHOUDHURY, D. A Near-Wall Treatment Using Wall Functions Sensitized to Pressure Gradient. In ASME FED Vol. 217, Separated and Complex Flows. ASME. 1995.

KOHLER, J. A. and STANER, K. E. High performance heat transfer surfaces. In Handbook of Applied Thermal Design, E. C. Guyer, Ed., pp. 7.37-7.49, McGraw-Hill, New York, NY, USA, 1984.

KROSE, B., SMAGT, P. V. D. An introduction to Neural Network. University of Amsterdam Press, 1996. 
KUROE, Y. AND KIMURA, I. Modeling of unsteady heat conduction field by using composite recurrent neural networks, Proc. IEEE Int. Conf. Neural Networks, Part 1, Vol. 1, pp. 323-328, 1995.

LAUNDER, B. E. and SPALDING, D. B. Lectures in Mathematical Models of Turbulence. Academic Press, London, England. 1972.

LAUNDER, B. E. AND SPALDING, D. B. The Numerical Computation of Turbulent Flows. Comp. Meth. in Applied Mechanics and Engineering. 3. 269-289, 1974.

LAVRIC, D., LAVRIC, V., MUNTEAN, O., AND DANCIU, E. Auto-organizing algorithm for design of fin heat exchangers, Rev. Roum. Chim., 39(11), 12411256, 1994.

LAVRIC, D., LAVRIC, V., AND WOINAROSCHY, A. Designing fin heat exchanger with a neural network, Rev. Roum. Chim., 10(2), 561-565, 1995.

LEI, Y.G., HE, Y.L., TIAN, L.T., CHU, P., TAO, W.Q. Hydrodynamics and heat transfer characteristics of a novel heat exchanger with delta-winglet vortex generators. Chem Eng Sci;65(5):1551-62, 2010.

LEMOUEDDA, A., BREUER, M., FRANZ, E., BOTSCH, T., DELGADO, A.. Optimization of the angle of attack of delta-winglet vortex generators in a platefin-and-tube heat exchanger. International Journal of Heat and Mass Transfer 53, 5386-5399, 2010.

LEHTOKANGAS, M., SAARINEN, J., AND KASKI, K. Initializing weights of a multilayer perceptron network by using the orthogonal least square algorithm, Neural Comput., 7, 982-999, 1995.

LEU, J.S., WU, Y.H., JANG, H.Y. Heat transfer and fluid flow analysis in plate-fin and tube heat exchangers with a pair of block shape vortex generators. Int $\mathrm{J}$ Heat Mass Transf; 47(19-20):4327-38, 2004.

LI, H.M., YE, K. S., TAN, Y. K. and DENG, S. J. Investigation on tube-side flow visualization, friction factors and heat transfer characteristics of helical-ridging tubes. In Proceedings of the 7th International Heat Transfer Conference, vol. 3, pp. 75-80, Munich, Germany, 1982.

LI, X., VAEZI-NEJAD, H., AND VISIER, J.-C. Development of a fault diagnosis method for heating systems using neural networks, ASHRAE Trans., 102(1), 607-614, 1996.

LI, H.Y. AND YANG, C.Y. A genetic algorithm for inverse radiation problems, Int. J. Heat Mass Transfer, 40(7), 1545, 1997.

LI, J., WANG, S., CHEN, J., LEI, Y-G. Numerical study on a slit fin-and-tube heat exchanger with longitudinal vortex generators. International Journal of Heat and Mass Transfer 54, 1743-1751, 2011.

MAHAJAN, R.L. AND WANG, X.A. Neural network models for thermally based microelectronic manufacturing processes, J. Electrochem. Soc., 140(8), 1993. 
MALISKA, C. R. Transferência de calor e mecânica dos fluidos computacional. $2^{a}$ edição revista e ampliada, LTC, Rio de Janeiro/RJ, 2004.

MANIAR, N. C. heat transfer enhancement in a rectangular channel using vortex generator in a laminar flow. Master Dissertation of Science in Mechanical Engineering, University of Texas, Arlington, 2012.

MATSUMOTO, R., KIKKAWA, S., AND SENDA, M. Effect of pin fin arrangement on endwall heat transfer, Trans.Jpn.Soc. Mech. Eng.B, 62(597), 1953-1961, 1996.

MATSUMOTO, R., KIKKAWA, S., AND SENDA, M. Effect of pin fin arrangement on endwall heat transfer, JSME Int. J.B: Flui.Thermal Eng., 40(1), 142-151, 1997.

McKAY, M. D., CONOVER, W. J., BECKMAN, R.J. A comparison of three methods for selecting values of input variables in the analysis of output from a computer code. Technometrics;21(2):239-45, 1979.

MENTER F. R. Two-Equation Eddy-Viscosity Turbulence Models for Engineering Applications. AIAA Journal. 32(8). 1598-1605. August 1994.

MEYSENC, L., SALUDJIAN, L., BRICARD, A., RAEL, S., AND SCHAEFFER, C. High heat flux IGBT micro exchanger setup, IEEE Trans. Components Package. Manuf. Technol. A, 20(3), 334-341, 1997.

MIN, C., QI, C., WANG, E., TIAN, L., QIN, Y. Numerical investigation of turbulent flow and heat transfer in a channel with novel longitudinal vortex generators. International Journal of Heat and Mass Transfer 55, 7268-7277, 2012.

MICHALEWICZ, Z. Genetic Algorithms + Data Structures = Evolution Programs, 2nd ed., Springer-Verlag, Berlin, 1992.

MITCHELL, M. An Introduction to Genetic Algorithms, MIT Press, Cambridge, 1997.

MOHAMAD, A. A. Heat transfer enhancements in heat exchangers fitted with porous media. Part I: constant wall temperature. International Journal of Thermal Sciences, vol. 42, no. 4, pp. 385-395, 2003.

MOMOSE, K., MURAI, T., ASAMI, T., and HOSOKAWA, Y. Identification of heatsource using neural network, Trans. Jpn. Soc. Mech. Eng. C, 59(567), 34313436, 1993.

MOON, C. AND CHO, B. Design of fuzzy control system for chemical injection system retrofit using neural network model in thermal power plant, Proc. 1st Int. Workshop Neural Networks Identification, Control, Robotics, and Signal/Image Process., pp. 263-269, 1996.

NAITO, K., YANG, J.-M., XU, Y. and KAGAWA Y. Enhancing the thermal conductivity of polyacrylonitrile- and pitch based carbon fibers by grafting carbon nanotubes on them. Carbon, vol. 48, no. 6, pp. 1849-1857, 2010.

NAKA, M., IMAI, T., SHIDA, T., SATO, M., ITO, RY, AND AKAMINE, I. Thermal image processing using neural network, Proc. Int. Joint Conf. Neural Networks, Part 3, Vol. 3, Nagoya, Japan, pp. 2065-2068, 1993. 
BARDINA, J. E., HUANG, P. G., COAKLEY, T. J. Turbulence Modeling Validation, Testing, and Development, NASA Technical Memorandum 110446, 1997.

NIST/SEMATECH. e-Handbook of Statistical Methods, 2011. http://www.itl.nist.gov/div898/handbook/.

NORMANDIN, A., GRANDJEAN, B.P.A., AND THIBAULT, J. PVT data analysis using neural network models, Ind. Eng. Chem. Res., 32(5), 970-975, 1993.

OKAMOTO, K., TSURU, D., AND FUMIZAWA, M. Reconstruction of threedimensional density distribution from the limited projection images with genetic algorithm, J. Flow Visual. Image Process., 3(4), 253, 1996.

ORSZAG, S. A., YAKHOT V., FLANNERY W. S., BOYSAN F., CHOUDHURY D., MARUZEWSKI J., and PATEL, B. Renormalization Group Modeling and Turbulence Simulations. In International Conference on Near-Wall Turbulent Flows, Tempe, Arizona. 1993.

PAPILA, M., HAFTKA, R. Response Surface Approximations: Noise, Error Repair, and Modeling Errors. AIAA/ASME/ASCE/AHS/ASC 40th. Structures, Structural Dynamics, and Materials Conference, St. Louis, MO, 2000.

PATANKAR, S. V. Numerical Heat Transfer and Fluid Flow, McGraw-Hill Book Co., New York, 1980.

PESTEEI, S.M., SUBBARAO, P.M.V., AGARWAL, R.S. Experimental study of the effect of winglet location on heat transfer enhancement and pressure drop in fintube heat exchangers. Applied Thermal Engineering 25, 1684-1696, 2005.

PICAN, N., ALEXANDRE, F., HATON, J.-P., AND BRESSON, P. Neurocontrol of temperatures with OWE neural network architecture in continuous steel annealing furnace, in Industrial Applications of Neural Networks, (Eds.) F.F. Soulie, P. Gallinari, World Scientific, Singapore, pp. 9-15, 1998.

QUEIPO, N., DEVARAKONDA, R., AND HUMPHREY, J.A.C. Genetic algorithms for thermosciences research: application to the optimized cooling of electronic components, Int. J. Heat Mass Transfer, 37(6), 893, 1994.

RAO, S. S. Engineering optimization: Theory and Practice. A Wiley-Interscience Publication John Wiley \& Sons, Inc., Third Edition, New York, 1996.

RASMUSSEN, C. E., WILLIAMS, C. K. I. Gaussian Processes for Machine Learning MIT Press, 2006.

RAUDENSKY, M., WOODBURY, K.A., AND BREZINA, T. Genetic algorithm in solution of inverse heat conduction problems, Num. Heat Transfer B, Fundam., 28(3), 293, 1995.

RICH, E. AND KNIGHT, K. Artificial Intelligence, 2 $2^{\text {nd }}$ ed., McGraw-Hill, NY, 1991.

ROGERS, J.I. Simulating structure analysis with neural networks, ASCE J. Comput. Civil Eng., 8(2), 252-265, 1994. 
RUMELHART, D.E., HINTON, D.E., AND WILLIAMS, R.J. Learning internal representations by error propagation, in Parallel Distributed Processing: Exploration in the Microstructure of Cognition, Vol. 1, Cambridge, MA, 1986.

RUSSEL, C. M. B., JONES, T. V. and LEE, G. H. Heat transfer enhancement using vortex generators, Heat Transfer 1982, Proc Seventh Heat Transfer Conf, 3: 283 \pm 288 , (Hemisphere, New York), 1982.

RUSSELL, S., NORVIG, P. Artificial intelligence : a modern approach. By PranticeHall, Inc. A Simon \& Schuster Company Englewood Cliffs, New Jersey, 1995.

RZEMPOLUCK, E.J. Neural Network Data and Analysis Using Simulnet, SpringerVerlag, New York, 1998.

SCHALKOFF R. Artificial Neural Networks, McGraw-Hill, New York, 1997.

SCHMIT, T.S., DHINGRA, A.K., LANDIS, F., AND KOJASOY, G. Genetic algorithm optimization technique for compact high intensity cooler design, J. Enhanced Heat Transfer, 3(4), 281-290, 1996.

SCHLICHTING, H. Boundary-layer Theory, 7th edn, McGraw-Hill, New York, 1979.

SIDDIQUE, M., KHALED, A. R. A., ABDULHAFIZ, N. I., and BOUKHARY, A. Y. Recent Advances in Heat Transfer Enhancements: A Review Report. Hindawi Publishing Corp. Int. J. of Chemical Eng.. Article ID 106461, 28 pages, 2010.

SHAH, R. K. Classification of Heat Exchangers. Thermal-Hydraulic Fundamentals and Design. Hemisphere Publishing Corporation, 1981.

SHAH, R. K. Compact Heat Exchangers for the Process Industries. Begell House, Inc. New York, 1997.

SOMMERS, A.D., JACOBI, A.M. Air-side heat transfer enhancement of a refrigerator evaporator using vortex generation. Int J Refrigeration;28(7):1006-17, 2005.

SONG, K-W., WANG, L-B. The Effectiveness of Secondary Flow Produced by Vortex Generators Mounted on Both Surfaces of the Fin to Enhance Heat Transfer in a Flat Tube Bank Fin Heat Exchanger. J. Heat Transfer, ASME, Vol. 135, 2013.

SPALDING, D. B.. GENMIX-A General Computer Program for Two-Dimensional Parabolic Phenomena, Pergamon, New York, 1977.

SUJUDI, D., HAIMES, R. Identification of Swirling Flow in 3-D Vector Fields, AIAA95-1715, 1995.

TAKEMORI, T., MIYASAKA, N., AND HIROSE, S. Neural network air-conditioning system for individual comfort, Proc. SPIE Int. Soc. Optical Eng. Appl. Artificial Neural Networks II, Vol. 1469, pt 1, pp. 157-165, 1991.

TANG, L.H., ZENG, M., WANG, Q.W. Experimental and numerical investigation on air-side performance of fin-and-tube heat exchangers with various fin patterns. Experimental Thermal and Fluid Science 33, 818-827, 2009. 
TAO, W.Q., HE, Y.L., LI, Z.Y., QU Z.G. Some recent advances in finite volume approach and their applications in the study of heat transfer enhancement. International Journal of Thermal Sciences 44, pp623-643, 2005.

TANIMOTO, S.L. The Elements of Artificial Intelligence Using Common LISP, Computer Science Press, New York, 1990.

THIBAULT, J. and GRANDJEAN, B.P.A. Neural network methodology for heat transfer data analysis, Int. J. Heat Mass Transfer, 34(8), 2063-2070, 1991.

TIGGELBECK, S., MITRA, N. AND FIEBIG, M. Flow Structure and Heat Transfer in a Channel with Multiple Longitudinal Vortex Generators. Exp. Thermal Fluid Science 5, p425-436, 1992.

TING, J. and LAKE, M. L. Vapor grown carbon fiber reinforced aluminum composites with very high thermal conductivity. Journal of Materials Research, vol. 10, no. 2, pp. 247-250, 1995.

TORII, K., KWAK, K.M., NISHINO, K. Heat transfer enhancement accompanying pressure loss reduction with winglet-type vortex generators for fin-tube heat exchangers. Int J Heat Mass Transf;45(18):3795-801, 2002.

VANDOORMAAL, J. P. AND RAITHBY, G. D. Enhancements of the SIMPLE Method for Predicting Incompressible Fluid Flows. Num. Heat Transfer 7, 1984.

VERSTEEG, H K, MALALASEKERA, W. An Introduction to Computational Fluid Dynamics, The Finite Volume Method. Second edition, Pearson Education Limited, Essex, England, 2007.

VON WOLFERSDORF, J., ACHERMANN, E., AND WEIGAND, B. Shape optimization of cooling channels using genetic algorithms, ASME J. Heat Transfer, 119(2), 380, 1997.

XIE, G., SUNDEN, B., WANG, Q., TANG, L. Performance predictions of laminar and turbulent heat transfer and fluid flow of heat exchangers having large tubediameter and large tube-row by artificial neural networks. International Journal of Heat and Mass Transfer 52, 2484-2497, 2009.

WANG, C-C., CHI, K-Y., CHANG, C-J. Heat transfer and friction characteristics of plain fin-and-tube heat exchangers, part II: Correlation. International Journal of Heat Mass Transfer 43, 2693-2700, 2000.

WANG, C-C., LO, J., LIN, Y-T., LIU, M-S. Flow visualization of wave-type vortex generators having inline fin-tube arrangement. International Journal of Heat and Mass Transfer 45, 1933-1944, 2002.

WARWICK, K., IRWIN, G.W., AND HUNT, K.J. Neural Networks for Control and Systems, Short Run Press, Exeter, U.K, 1992.

WEBB, R. L. Principles of enhanced heat transfer. Published by Taylor \& Francis Group 270 Madison Avenue, New York, NY, 2005. 
WESSELS, L. and BARNARD, E. Avoiding fake local minima by proper initialization of connections, IEEE Trans. Neural Networks, 3(6), 899-905, 1992.

WILCOX, D. C. Turbulence Modeling for CFD. DCW Industries, Inc. La Canada, California. 1998.

WOOD, R.L. Inverse thermal field problem based on noisy measurements: comparison of a genetic algorithm and the sequential function specification method, Eng. Computational. 13(6), 43-59, 1996.

WU, J.M., TAO, W.Q. Investigation on laminar convection heat transfer in fin-andtube heat exchanger in aligned arrangement with longitudinal vortex generator from the viewpoint of field synergy principle. J Appl Therm Eng 2007;27(1415):2609-17, 2007.

WU, J.M., TAO, W.Q. Impact of delta winglet vortex generators on the performance of a novel fin-tube surfaces with two rows of tubes in different diameters. Energy Conversion and Management 52, 2895-2901, 2011.

WU, J.M., ZHANG, H., YAN, C.H., WANG, Y. Experimental study on the performance of a novel fin-tube air heat exchanger with punched longitudinal vortex generator. Energy Conversion and Management 57, 42-48, 2012.

YANAGIHARA, J.I.; TORII, K. Enhancement of Laminar Boundary Layer Heat Transfer by Longitudinal Vortices, In: Transport Phenomena in Heat and Mass Transfer, Ed. J.A. Reizes, Elsevier Science Pub., Washington D.C., Vol. 2, pp. 942-953, 1991.

YANG, Q.-H., MA, H., LIU, X., AND SHI, H. Thermal deformation compensation for a coordinate grinder by neural networks, Proc. SPIE Int. Soc. Optical Eng. Int. Conf. Intelligent Manuf., pp. 499-502, 1995.

YENTIS, R., JR. AND ZAGHLOUL, M.E. CMOS implementation of locally connected neural cells to solve the steady-state heat flow problem, Proc. 37th Midwest Symp. Circuits Systems, Part 1, Vol. 1, Lafayette, LA, pp. 503-506, 1994.

ZENG, P. Neural Computing in Mechanics, Appl. Mech. Rev., 51(2), 173-197, 1998.

ZBICINSKI, I., STRUMILLO, P., AND KAMINSKI, W. Hybrid neural model of thermal drying in a fluidized bed, Computational. Chem. Eng., 20, S695-S700, 1996.

ZHANG, Y.H., WU, X., WANG, L.B., SONG, K.W., DONG, Y.X., LIU, S. Comparison of heat transfer performance of tube bank fin with mounted vortex generators to tube bank fin with punched vortex generators. Exp Thermal Fluid Science 2008;33(1):58-66, 2008

ZHOU, G., YE, Q. Experimental investigations of thermal and flow characteristics of curved trapezoidal winglet type vortex generators. Applied Thermal Engineering 37, 241-248, 2012. 


\section{A. CFD validation procedure}

The numerical procedure to solve the governing Computational Fluid Dynamic (CFD) equations was conducted by Finite Volume Methodology, already implemented into Ansys Fluent v.14.0.0 environment.

Pre-processing step was performed through two commercial software's; 2D-modeling of the computational domain was discretized by Hypermesh v.10.0, applying a two-dimensional grid generator, and T-Grid v.5.0.6 was applied to generate the threedimensional tetrahedral unstructured grid. The mesh density and structure have a significant influence on stability and accuracy of the numerical solution. Thus, the optimum mesh should be fine enough to reduce the discretization error and solve the flow field, especially in high gradients zones, enabling the identification of the mean patterns of the flow. The grid generation strategy was determined as a function of the size and location of flow features, such as: boundary layers, mixing zones, separated regions and shear layers. For wall-bounded flows, the grid size at the wall can influence the accuracy of the computed shear stress and heat transfer coefficient.

Post-processing step was made using commercial software called by Ensight. This tool is an easily way to find the vortical structures produced by VG. Origin v.8.6 was considered in post-processing step for graphics analysis.

A great facility that enabled the optimization process was the High Performance Computing place available to Ford CFD Team, which contains more than 5000 CPUs available. It is very useful because the big account jobs necessary for optimization process and the time needs for run each job.

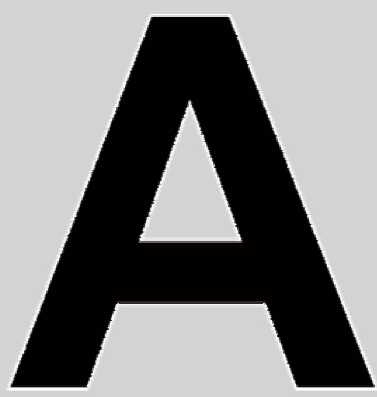


Automatic process was made by the commercial software called by ModeFrontier. This tool is an easily way for integration among each step, ie, pre-processing (Hypermesh + TGrid) and processing (FLUENT - solver). This software was also applied to extract quickly the mean results (average temperature and pressure at inlet/outlet) from run jobs to data-sheet. These features turn possible the metamodeling and optimization process in an average short time comparing to the conventional approach.

Thus, regarding the summary in Table 5 and Table 6, it was possible to go to validation procedure. The correlation by Wang et al. (2000) was considered. The Friction factor $(\mathrm{f})$ is shown in Equation 87 and Colburn factor $(\mathrm{j})$ in Equation 88.

$$
f=0.0267 \operatorname{Re}_{D_{c}}^{F 1}\left(\frac{P_{T}}{P_{L}}\right)^{F 2}\left(\frac{F_{P}}{D_{c}}\right)^{F 3}
$$

where:

$\operatorname{Re}_{D_{c}} \rightarrow$ Reynolds number based on fin collar outside diameter.

$F 1=-0.764+0.739 \frac{P_{T}}{P_{L}}+0.117 \frac{F_{P}}{D_{c}}-\frac{0.00758}{N}$

$F 2=-15.689+\frac{64.021}{\log _{e}\left(\operatorname{Re}_{D_{c}}\right)}$

$\mathrm{F} 3=1.696-\frac{15.695}{\log _{\mathrm{e}}\left(\operatorname{Re}_{\mathrm{D}_{\mathrm{c}}}\right)}$

And:

$$
j=0.086 \operatorname{Re}_{D_{c}}^{P 1} N^{P 2}\left(\frac{F_{P}}{D_{c}}\right)^{P 3}\left(\frac{F_{P}}{D_{h}}\right)^{P 4}\left(\frac{F_{P}}{P_{T}}\right)^{-0.93}
$$

where:

$D_{h} \rightarrow$ Hydraulic diameter $\left(4 A_{c} L / A_{o}\right)$ 


$$
\begin{aligned}
& P 1=-0.361-\frac{0.042 N}{\log _{e}\left(R_{D_{c}}\right)}+0.158 \log _{e}\left[N\left(\frac{F_{P}}{D_{c}}\right)^{0.41}\right] \\
& P 2=-1.224-\frac{0.076\left(\frac{P_{L}}{D_{h}}\right)^{1.42}}{\log _{e}\left(\operatorname{Re}_{D_{c}}\right)} \\
& P 3=-0.083+\frac{0.058 N}{\log _{e}\left(\operatorname{Re}_{D_{c}}\right)} \\
& P 4=-5.735+1.211 \log _{e}\left(\frac{R e_{D_{c}}}{N}\right)
\end{aligned}
$$

Figure 117 and Figure 118 show the results for Friction and Colburn factor for numerical simulation, according to Table 5, and Wang et al. (2000) correlation, Equation 87 and 88, respectively. Further, the average deviation (detail) for each parameter is also showed.

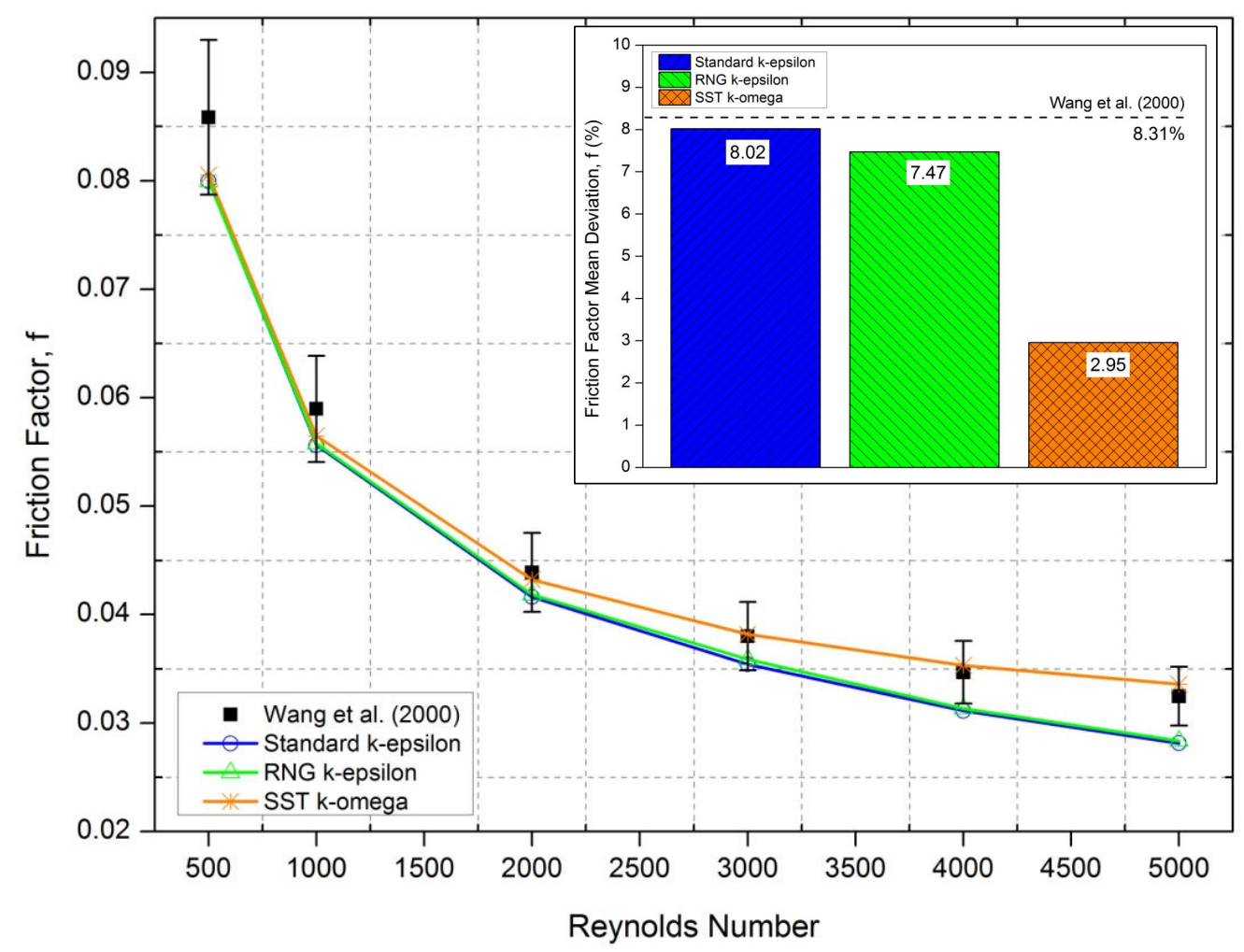

Figure 117 - Friction factor from Wang et al. (2000) correlation - Equation 87 - and the different numerical approaches. Without vortex generator. 


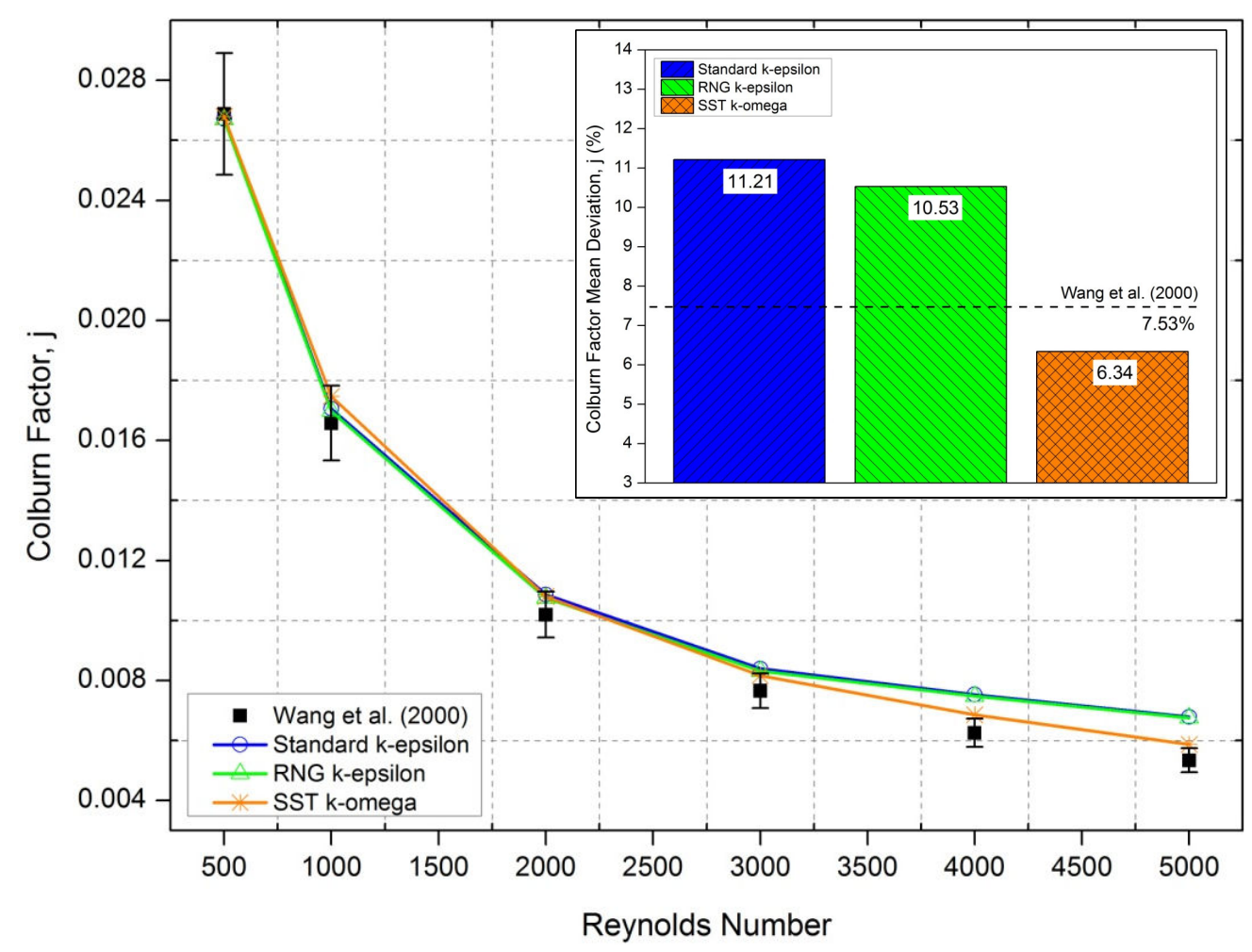

Figure 118 - Colburn factor from Wang et al. (2000) correlation - Equation 88 - and the different numerical approaches. Without vortex generator.

In order to verify the present numerical work, the model validation was conducted with help of a fin-and-tube heat exchanger without VG in staggered arrangement; therefore, the correlations from Wang et al. (2000) could be applied. Grid independence for heat exchanger with VG was also evaluated. Both Figure 117 and Figure 118 showed that the numerical simulation results tend to Wang correlations profile for all approaches considered herein, according to Table 5. However, evaluating the detail in Figure 117 and Figure 118, only SST k-omega approach presented an average deviation error lower than Wang correlations for both Friction and Colburn factors. For Standard and RNG k-epsilon approaches only Friction factor average deviation was lower than the Wang correlation, Equation 87. In most of researches, $\mathrm{k}$-epsilon model with SIMPLE pressure-velocity coupling algorithm was applied, and the main reason for this is the concern to convergence time and/or computational consumption expense. Regarding meta-modeling and the optimization process, this issue is a big concern because of the RSM quality. SST k-omega model spent more time for full convergence and in some cases was need a hard 
work on under-relaxation to momentum and energy equations. However, the accuracy of the SST k-omega model, Figure 117 and Figure 118, is unquestionable. Due to the thin channel and influence of the fin walls on the flow of air in the heat exchanger, the results in Figure 117 and Figure 118, and also the key characteristics of each turbulence model, discussed in the Chapter 3 , it was decided to carry out simulations using the SST $\mathrm{k}$-omega model.

Grid independence was also evaluated for both with and without VG, considering three different refine grid. Table 9 shows the numerical results compared to Wang et al. (2000) correlations (without VG); Table 10 and Table 11 show the results applying VG for the same computational domain considered for $\operatorname{Re}=250$ and $R e=1400$, respectively.

Table 9 - Summary of the grid independence without vortex generator.

\begin{tabular}{cccc}
\hline \multirow{2}{*}{$\begin{array}{c}\text { Cells number } \\
\text { (Full computational } \\
\text { domain, figure 42) }\end{array}$} & \multicolumn{2}{c}{ Average Deviation (\%) } \\
\cline { 2 - 4 } & $\begin{array}{c}\text { Friction } \\
\text { Factor, } \mathrm{f}\end{array}$ & $\begin{array}{c}\text { Colburn } \\
\text { Factor, } \mathrm{j}\end{array}$ \\
\hline GRID 1 & $6.010^{+5}$ & 6.13 & 11.81 \\
GRID 2 & $8.810^{+5}$ & 2.95 & 6.34 \\
GRID 3 & $14.710^{+5}$ & 2.82 & 5.66 \\
\hline
\end{tabular}

Table 10 - Summary of the grid independence with vortex generator for $\operatorname{Re}=250$.

\begin{tabular}{|c|c|c|c|}
\hline \multirow{2}{*}{$\begin{array}{c}\text { Cells number } \\
\text { (Full computational } \\
\text { Domain with VG) }\end{array}$} & \multicolumn{3}{|c|}{$\mathrm{Re}=250$} \\
\hline & $\begin{array}{l}\text { Friction } \\
\text { Factor, } f\end{array}$ & $\begin{array}{l}\text { Colburn } \\
\text { Factor, j }\end{array}$ & $\begin{array}{c}\bar{y}^{+} \\
\text {(Equation 49) }\end{array}$ \\
\hline GRID 1 & 0.074466 & 0.021812 & 0.67 \\
\hline GRID 2 & 0.074833 & 0.020665 & 0.61 \\
\hline GRID 3 & 0.074819 & 0.020321 & 0.46 \\
\hline
\end{tabular}

Table 11 - Summary of the grid independence with vortex generator for $\operatorname{Re}=1400$.

\begin{tabular}{cccc}
\hline Cells number & \multicolumn{3}{c}{$\mathrm{Re}=1400$} \\
\cline { 2 - 4 } $\begin{array}{c}\text { (Full computational } \\
\text { Domain with VG) }\end{array}$ & Friction & $\begin{array}{c}\text { Colburn } \\
\text { Factor, } \mathrm{j}\end{array}$ & $\begin{array}{c}\overline{\mathrm{y}}^{+} \\
\text {(Equation 49) }\end{array}$ \\
\hline GRID 1 & 0.040719 & 0.009209 & 2.25 \\
GRID 2 & 0.041705 & 0.008763 & 2.05 \\
GRID 3 & 0.041485 & 0.008623 & 1.13 \\
\hline
\end{tabular}


For both Colburn and Friction factors, Table 9 shows that the average deviation to GRID 2 was lower than the Wang correlation and for the GRID 3 the results not significantly decreased the error. Thus, the GRID 2 could be considered enough. However, evaluate the $\bar{y}^{+}$value and Colburn and Friction factor sensibility is also necessary. Table 10 and Table 11 show only $\bar{y}^{+}$for $\operatorname{Re}=250$ and $\operatorname{Re}=1400$, because the optimization process considered herein were for these two levels, ie, $\operatorname{Re}=250$ is the lower limit and $\operatorname{Re}=1400$ is the upper limit, according to application discussed in Chapter 1. From Table 10, evaluating $\operatorname{Re}=250$ and Colburn factor, the difference between GRID 1 and GRID 2 was 5.26\%, whereas between GRID 2 and GRID 3 was 1.66\%; for Friction factor, the difference was $0.49 \%$ and $0.19 \%$, respectively. For $\operatorname{Re}=1400$ and Colburn factor, the difference between GRID 1 and GRID 2 was 4.85\%, whereas between GRID 2 and GRID 3 was 1.59\%; for Friction factor, the difference was $2.42 \%$ and $0.53 \%$, respectively. These results show that the GRID 2 could also be enough for the objectives of this work. However, $\overline{\mathrm{y}}^{+}$value is an important metric and the Ansys Fluent (solver developer) recommend to work close to $1\left(\mathrm{y}^{+} \approx 1\right)$. Therefore, the GRID 3 should be the best option. On the other hand, the time consumption for each job simulation increased almost three times. Considering the computational resource available, this concern is not a problem and the GRID3 was chosen to optimization process.

Boundary condition for turbulence model SST k-omega must supply at inlet boundary (velocity inlet, pressure inlet, etc.). In many situations, specify correct or realistic boundary condition at the inlet could helps in convergence, beyond the inlet turbulence could result in significantly affect the downstream flow. Thus, the inlet turbulence intensity, calculated by Equation 48, was evaluated between $2 \%$ and $10 \%$. This turbulence intensity range is commonly reported in open literature. Figure 119 and 120 show the results for $\operatorname{Re}=250$ and $\operatorname{Re}=1400$, respectively.

For the application range defined in this work (inlet velocity between $1.0 \mathrm{~m} / \mathrm{s}$ and $6.0 \mathrm{~m} / \mathrm{s}$ ), Figure 119 and Figure 120 showed that the changes in the inlet turbulence intensity were not significant on Colburn and Friction factors. Thus, as input data for turbulence model, the Equation 48 was used as reference. 


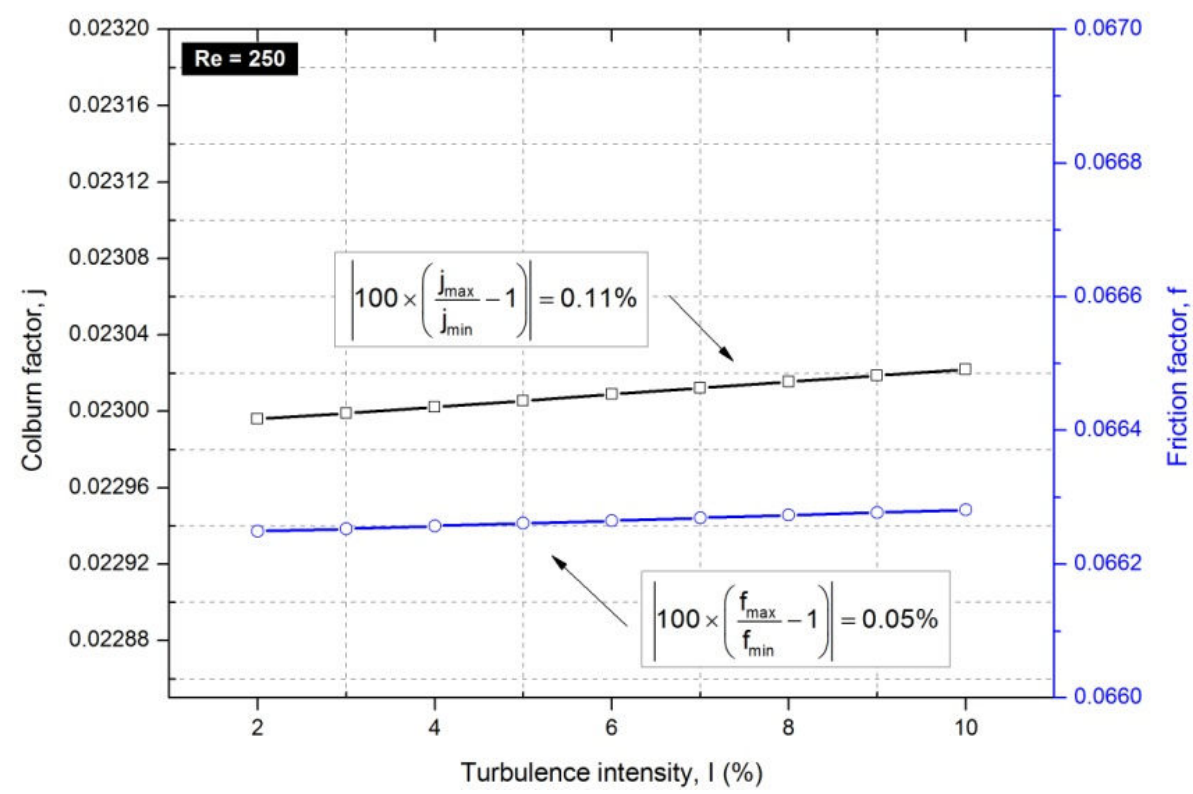

Figure 119 - Impact on inlet turbulence intensity in Colburn and Friction factor for $\mathrm{Re}=250$.

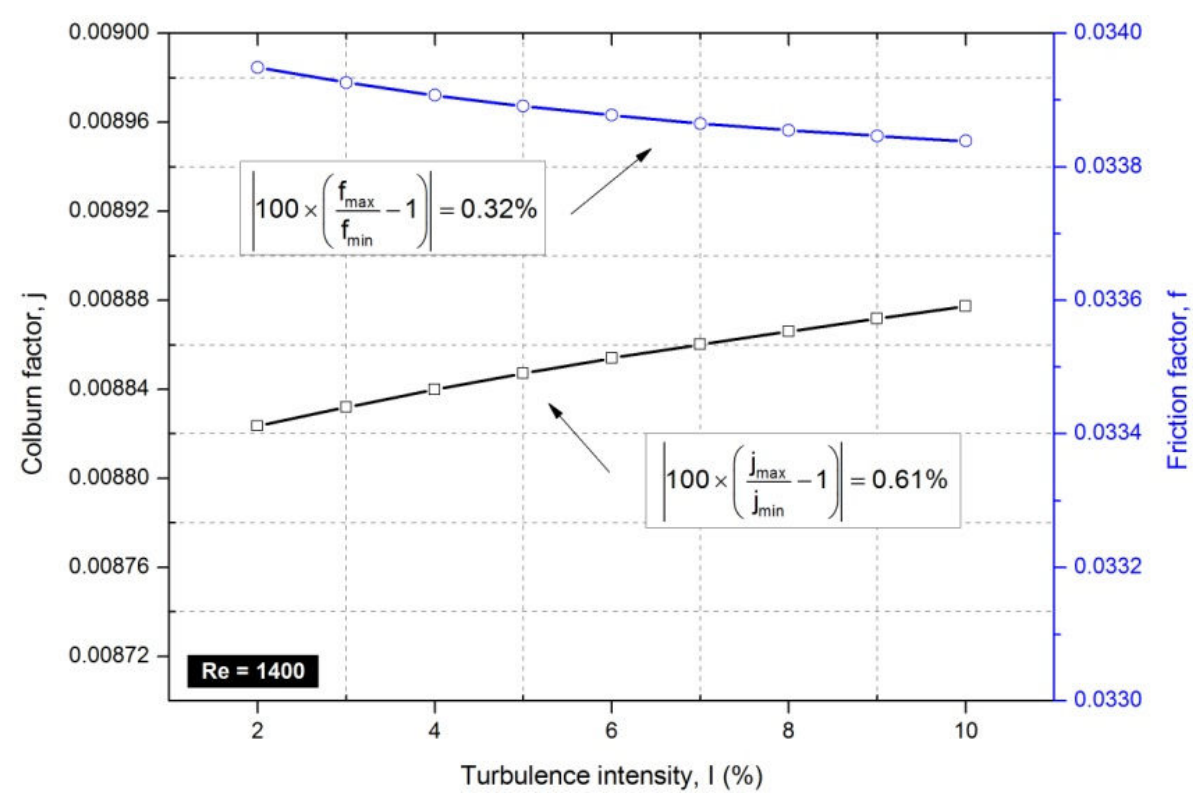

Figure 120 - Impact on inlet turbulence intensity in Colburn and Friction factor for $\operatorname{Re}=1400$.

Further, Figure 121 and Figure 122 show the local heat transfer coefficient, Equation 89, and relative pressure field for $\operatorname{Re}=250$ and $\operatorname{Re}=1400$, considering the numerical setup discussed in Chapter 3 and in this Appendix A. 


$$
\mathrm{h}=\frac{-\left.\mathrm{k}_{\mathrm{f}} \frac{\partial \mathrm{T}}{\partial \eta}\right|_{\eta=0}}{\mathrm{~A}_{\mathrm{o}}\left(\mathrm{T}_{\mathrm{s}}-\mathrm{T}_{\text {ref }}\right)}
$$

Where:

$\mathrm{k}_{\mathrm{f}} \rightarrow$ Fluid Thermal Conductivity $(\mathrm{W} / \mathrm{m} . \mathrm{K})$

$\mathrm{T}_{\mathrm{s}} \rightarrow$ Superficial temperature $(\mathrm{K})$

$\mathrm{T}_{\text {ref }} \rightarrow$ Referential temperature defined as inlet domain temperature $(303.15 \mathrm{~K})$

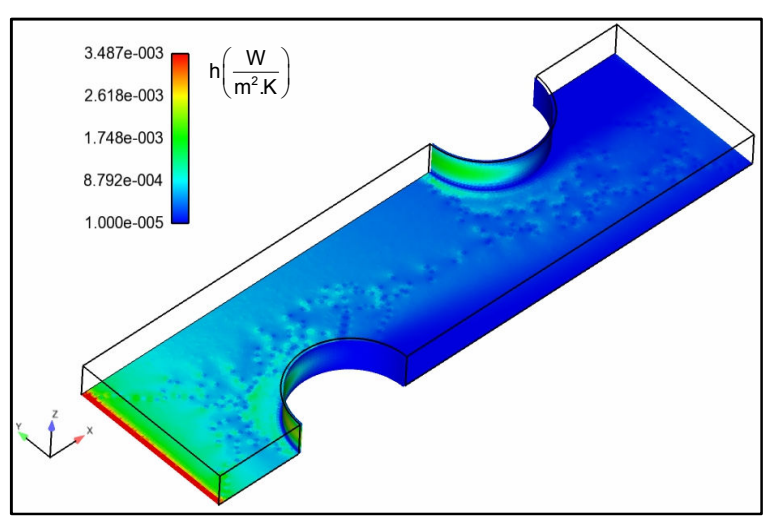

(a)

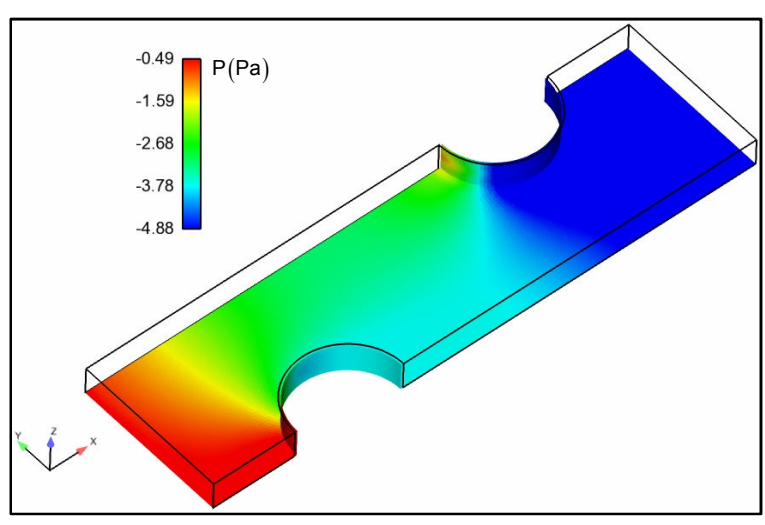

(b)

Figure 121 - Results for $\operatorname{Re}=250$ and SST k-omega turbulence model. (a) Heat transfer coefficient, (b) Relative pressure.

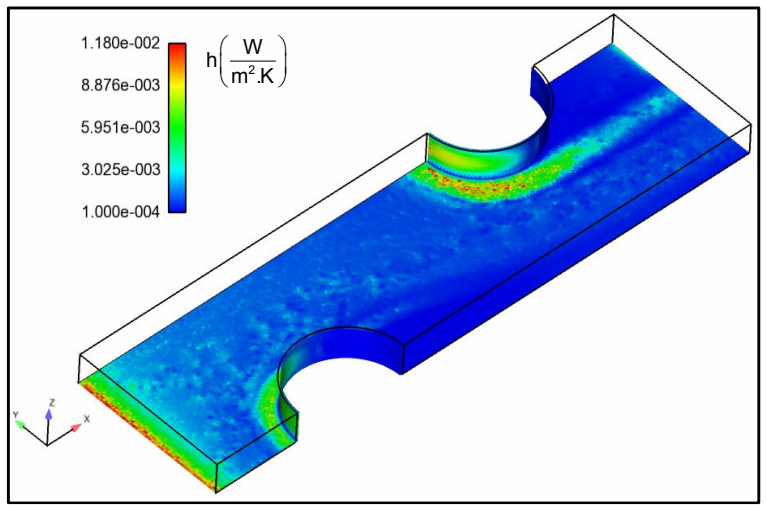

(a)

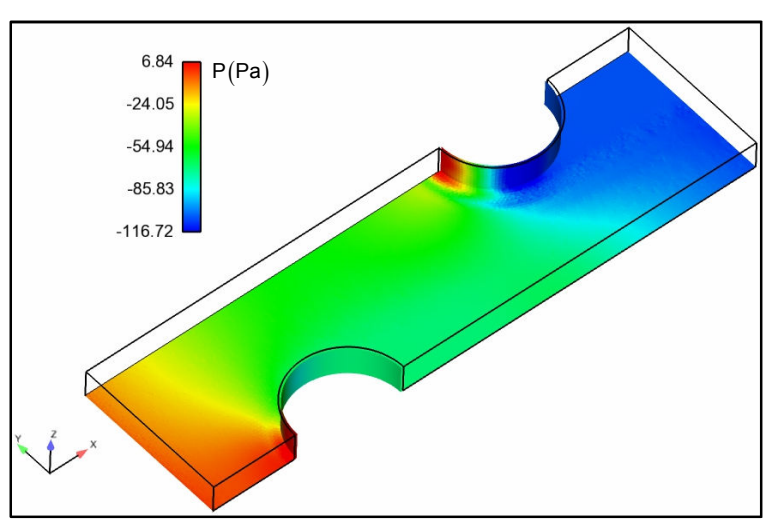

(b)

Figure 122 - Results for Re $=1400$ and SST k-omega turbulence model. (a) Heat transfer coefficient, (b) Relative pressure. 
These results are shown here for emphasize the reasons to believe that the VG for the first tube and second tube could generate different influence on heat transfer and pressure drop. Firstly, it was observed the different fields around the tubes. Figure 121a showed that the heat transfer was stronger around the first tube than the second tube. Thus, it is expected that the position and angles of the VG for the first and second tube ought to be asymmetry. Figure $121 \mathrm{~b}$ also showed significant difference in relative pressure field around the first and second tube, supporting the previous assessments. This same analysis was made for $\operatorname{Re}=1400$, considering the Figure 122a and Figure 122b. The heat transfer field and the relative pressure field were different for $\operatorname{Re}=250$ and $\operatorname{Re}=1400$, leading to conclusion that the position and angles of the VG could be different for each Reynolds number, as well as, for each tube. Further, for $\operatorname{Re}=1400$, Figure $122 \mathrm{a}$ showed that the heat transfer was stronger around the second tube than the first tube.

The only difference between the setup for validation procedure and setup for optimization process was the boundary condition for fin, ie, for validation procedure the conduction and convective heat transfer were simultaneously solved, whereas for optimization process the fin and tube temperature were constant. 


\section{B. Surrogate model validation}

This appendix is dedicated to present the RSM training and test results, beyond the comparison among different surrogate methods for RSM approach. Results reported herein complement and sustain the discussion previously made in Chapter 4. Regression definition, Equation 81, was conveniently repeated below.

$$
R^{2}=1-\frac{\sum_{i=1}^{n}\left(\phi_{i}-\tilde{\phi}_{i}\right)^{2}}{\sum_{i=1}^{n}\left(\phi_{i}-\bar{\phi}_{i}\right)^{2}}
$$

Figure 123 to 126 shown the Regression $\left(R^{2}\right)$, Mean Relative Error $\left(e_{\text {mean }}\right)$ and Maximum Relative Error $\left(e_{\max }\right)$ for different number of neurons at $\operatorname{Re}=250$ and $\operatorname{Re}=1400$. Forrester et al. (2008) suggest that the good results for RSM are observed for $\mathrm{R}^{2}>0.8, \mathrm{e}_{\max }<10 \%$ and $\mathrm{e}_{\text {mean }}<5 \%$.

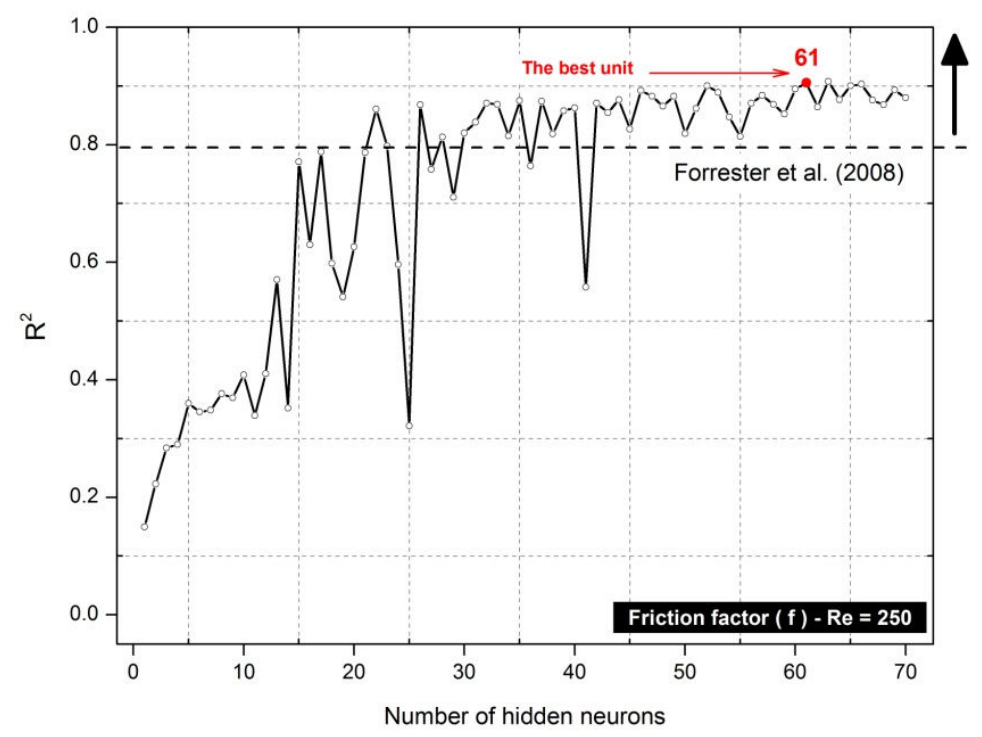

(a) 


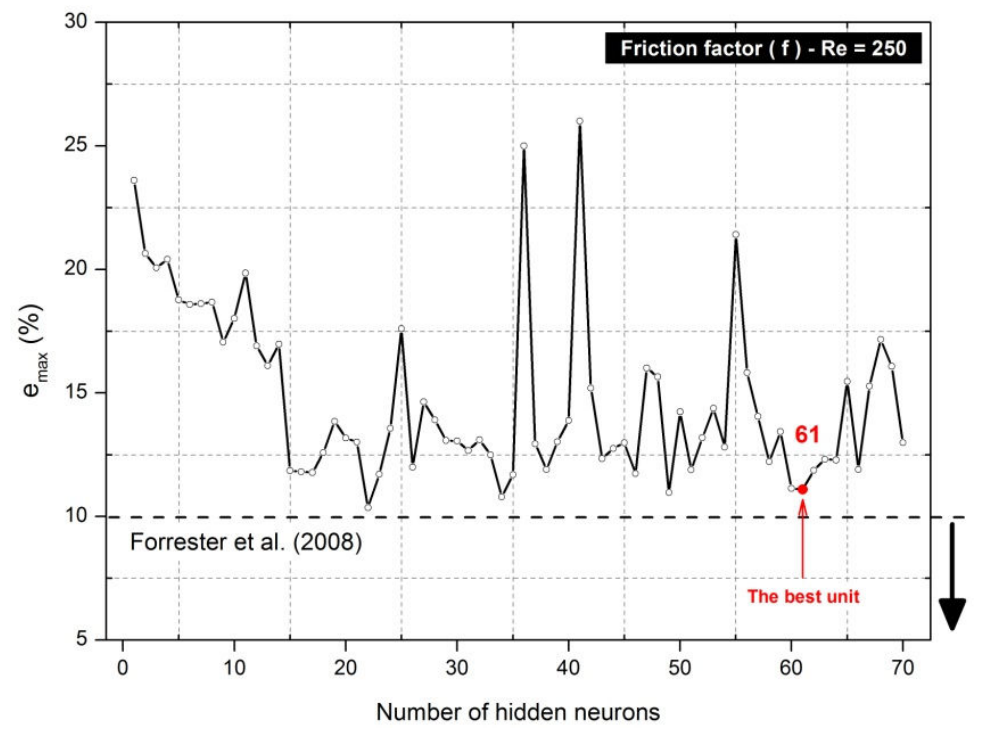

(b)

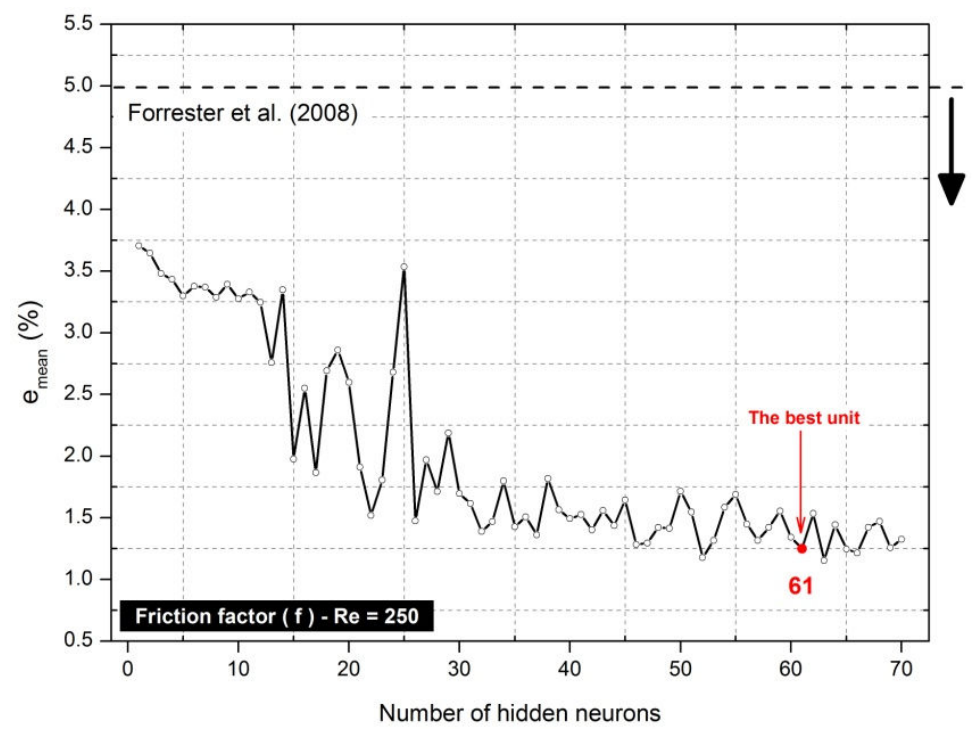

(c)

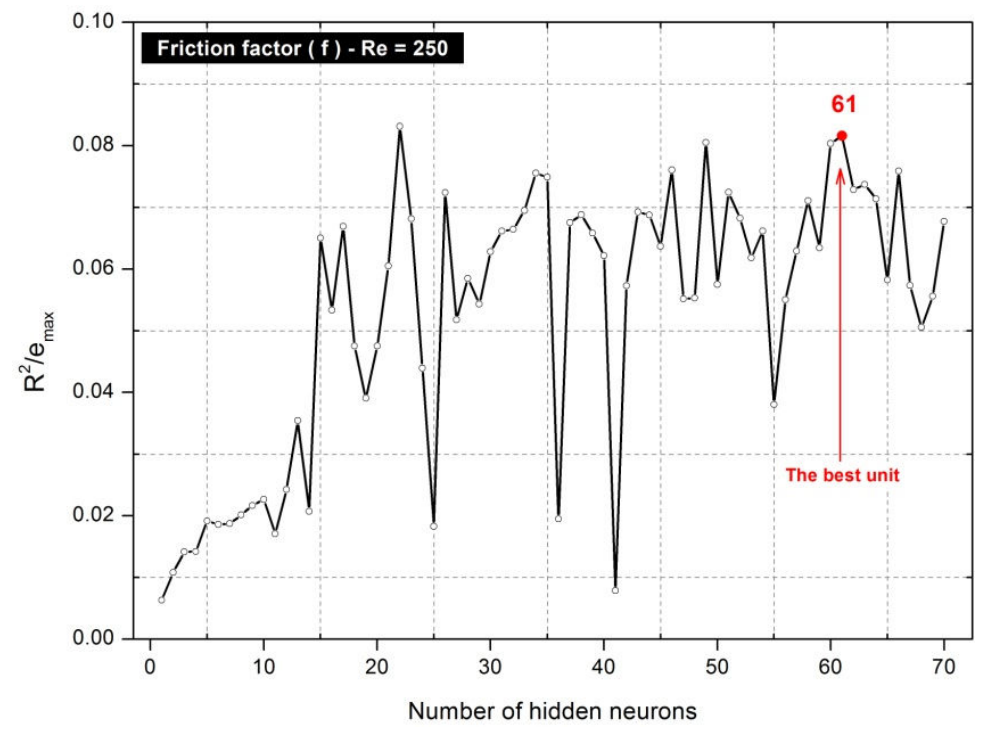

(d)

Figure 123 - RSM training error and regression as a function of the number of hidden neurons unit at $\mathrm{Re}=250$ for Friction factor (f). 


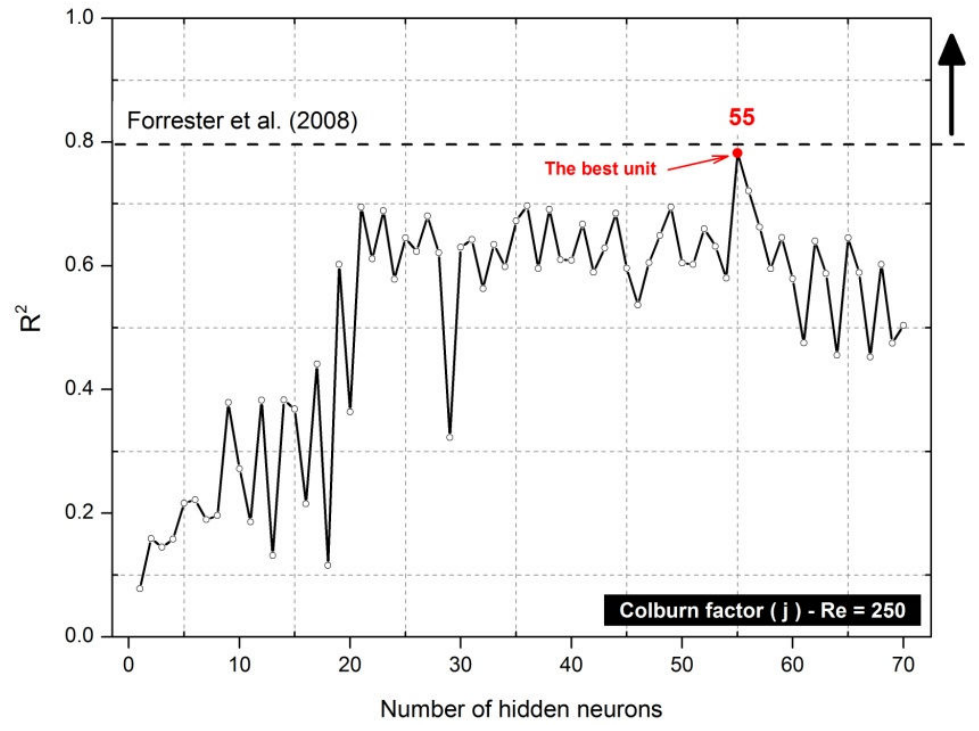

(a)

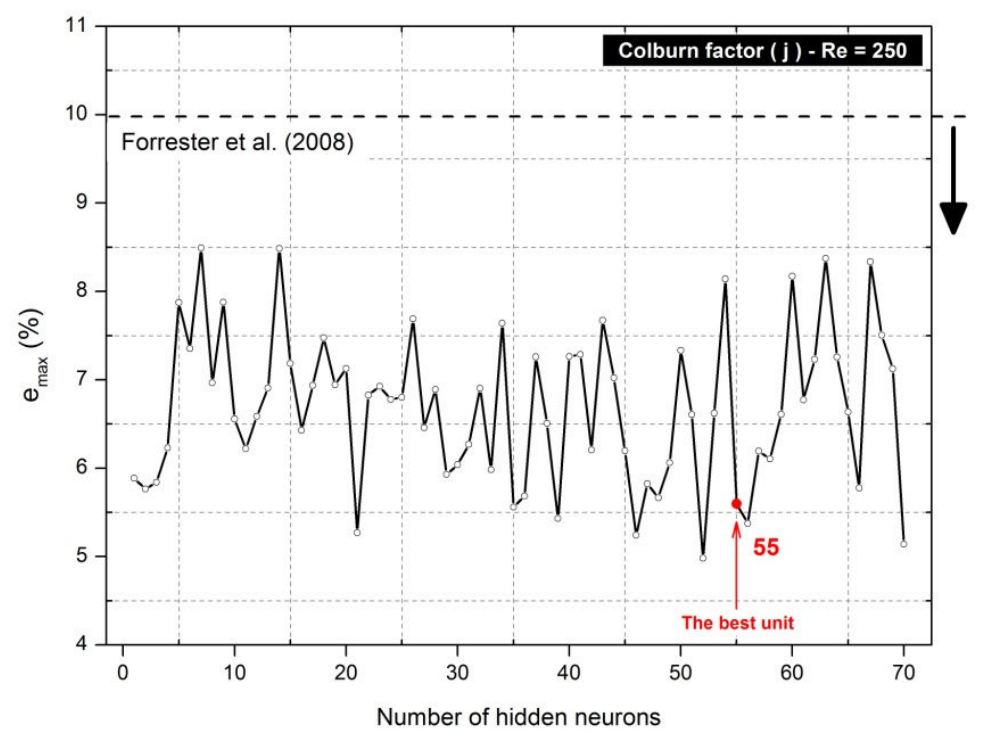

(b)

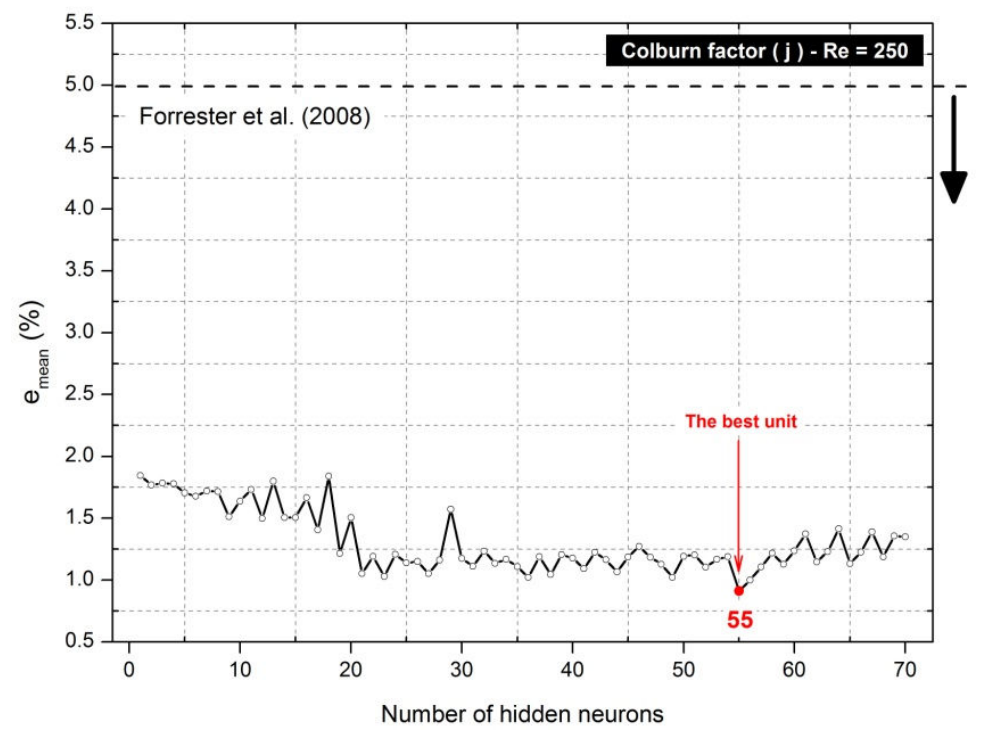

(c) 


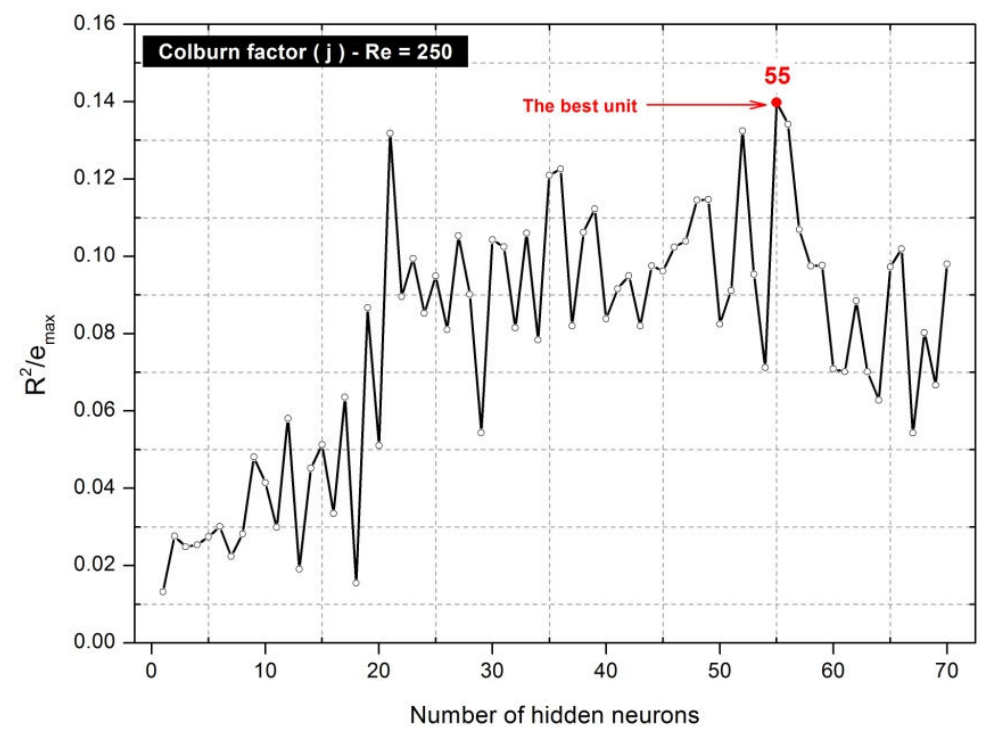

(d)

Figure $124-\mathrm{RSM}$ training error and regression at $\mathrm{Re}=250$ for Colburn factor (j).

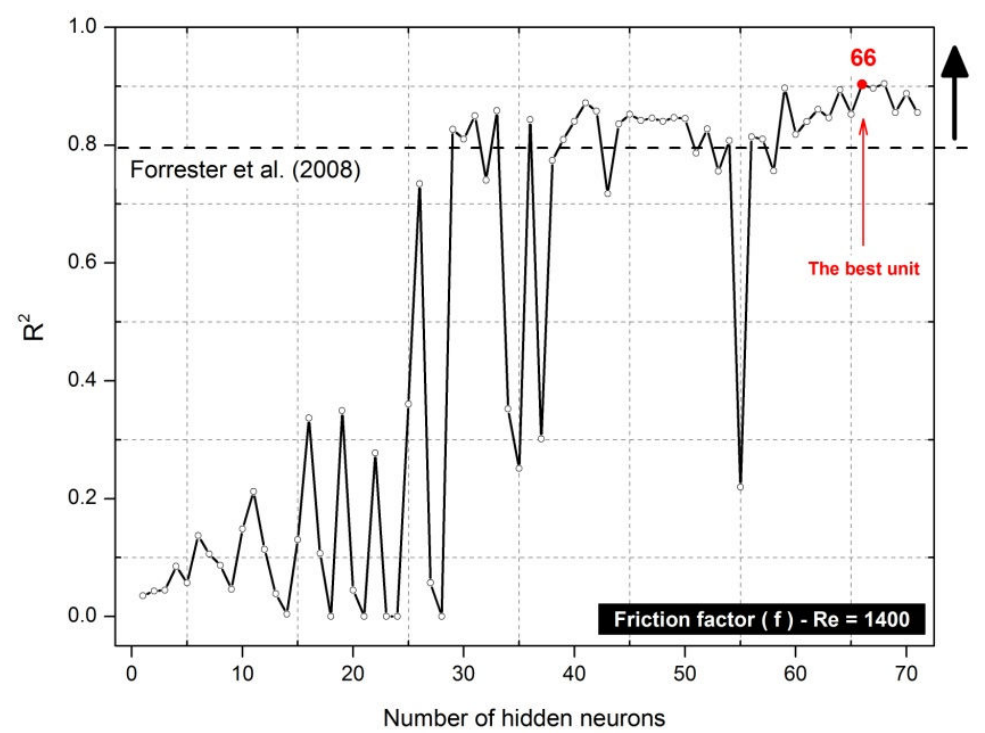

(a)

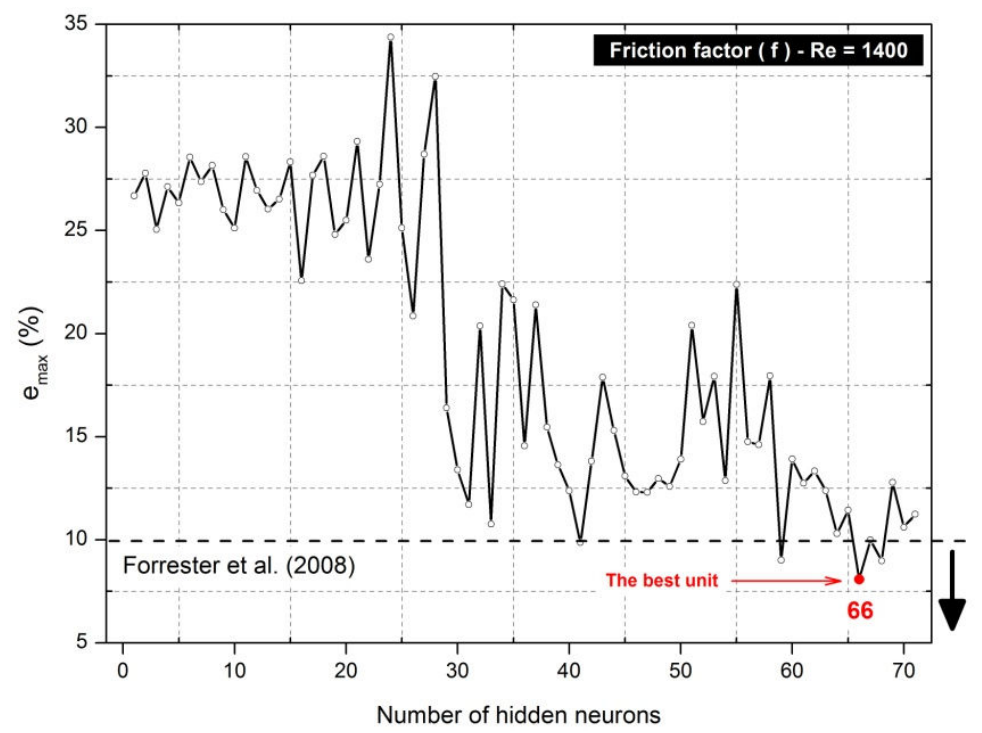

(b) 


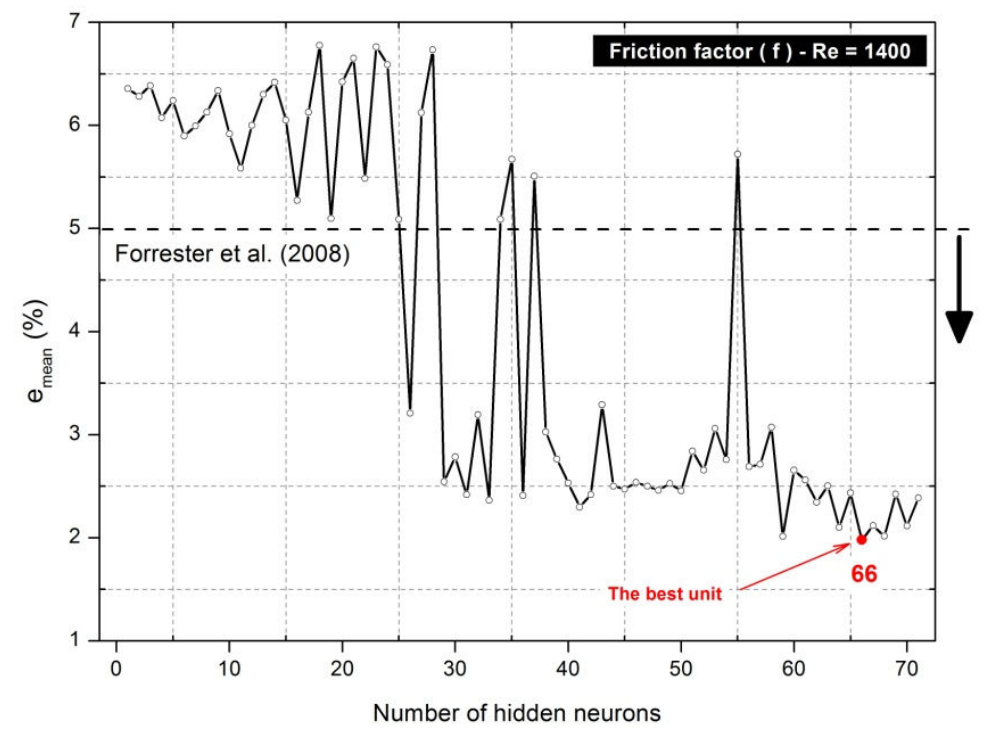

(c)

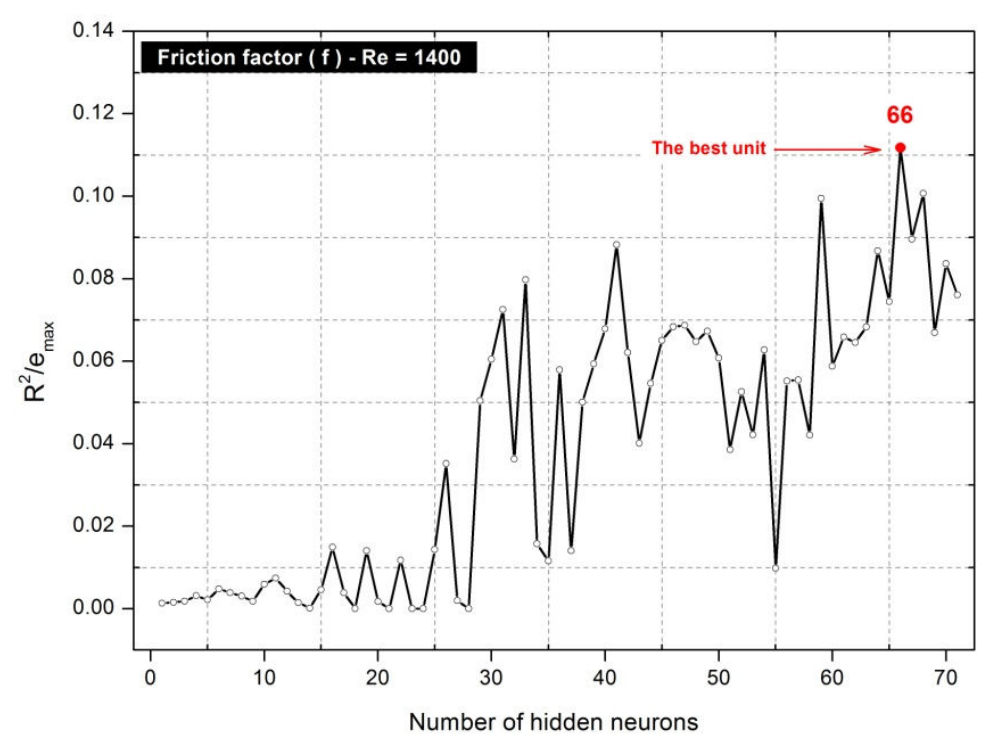

(d)

Figure $125-\mathrm{RSM}$ training error and regression at $\mathrm{Re}=1400$ for Friction factor $(\mathrm{f})$.

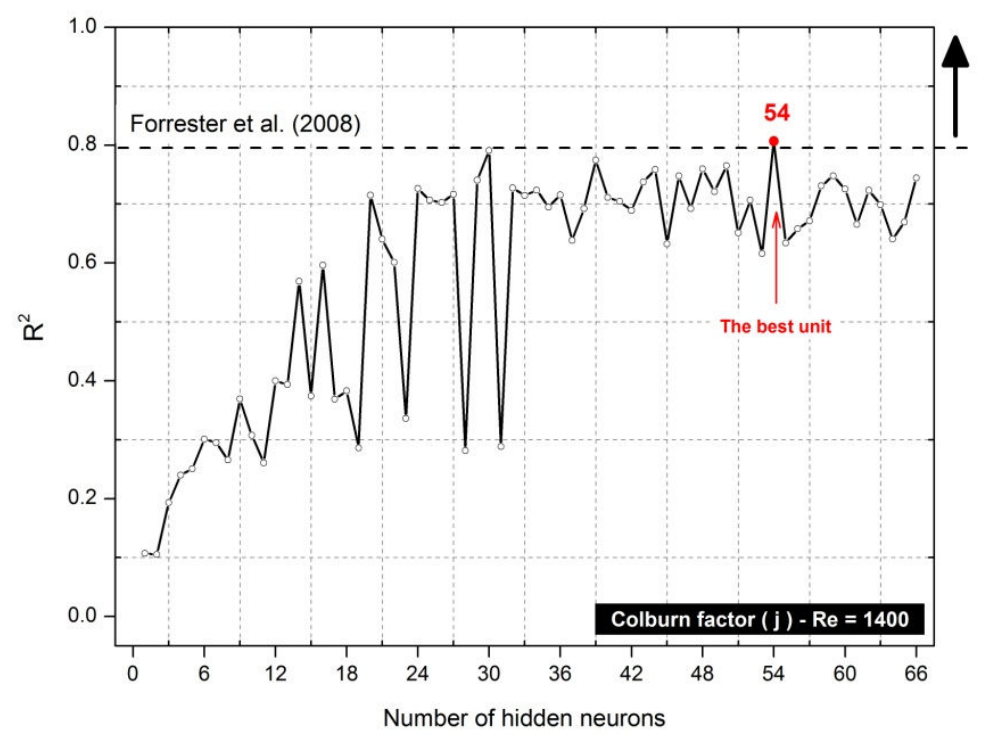

(a) 


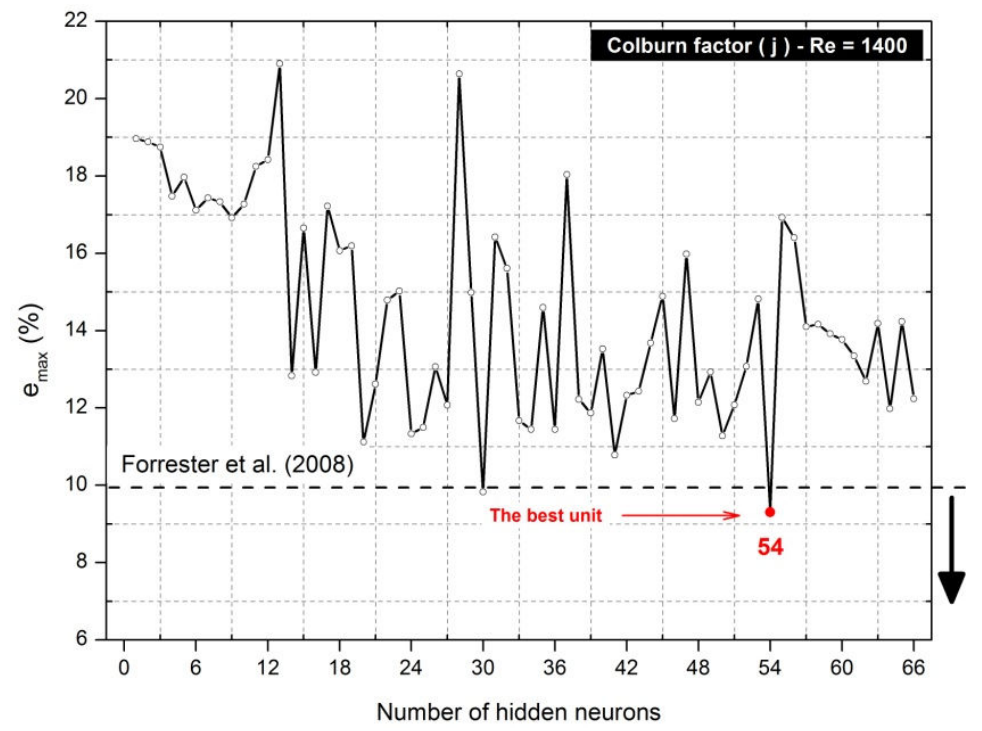

(b)

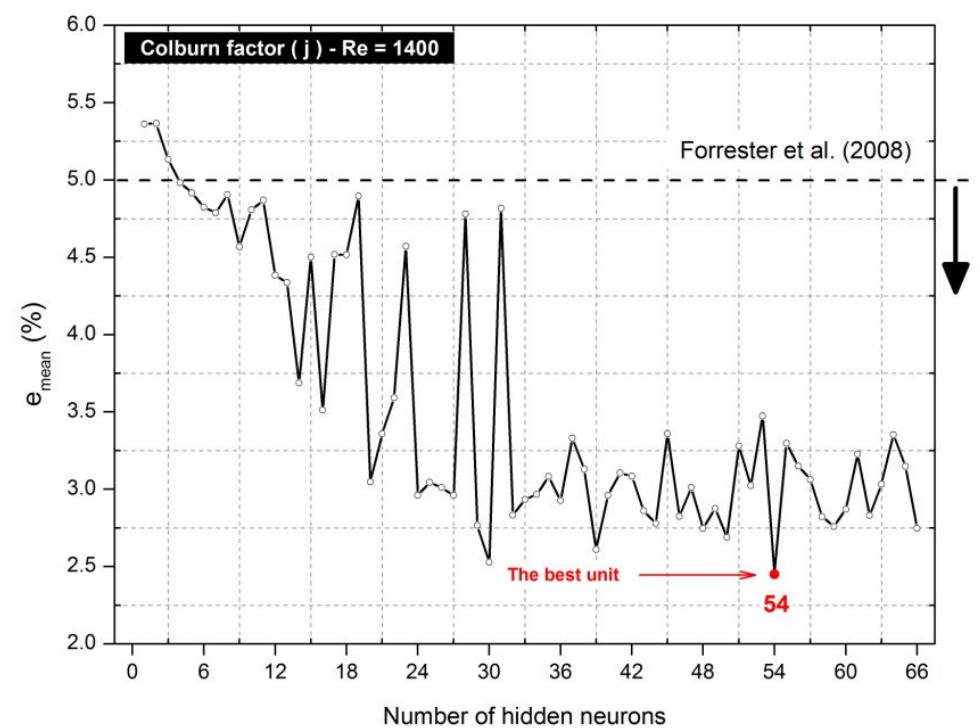

(c)

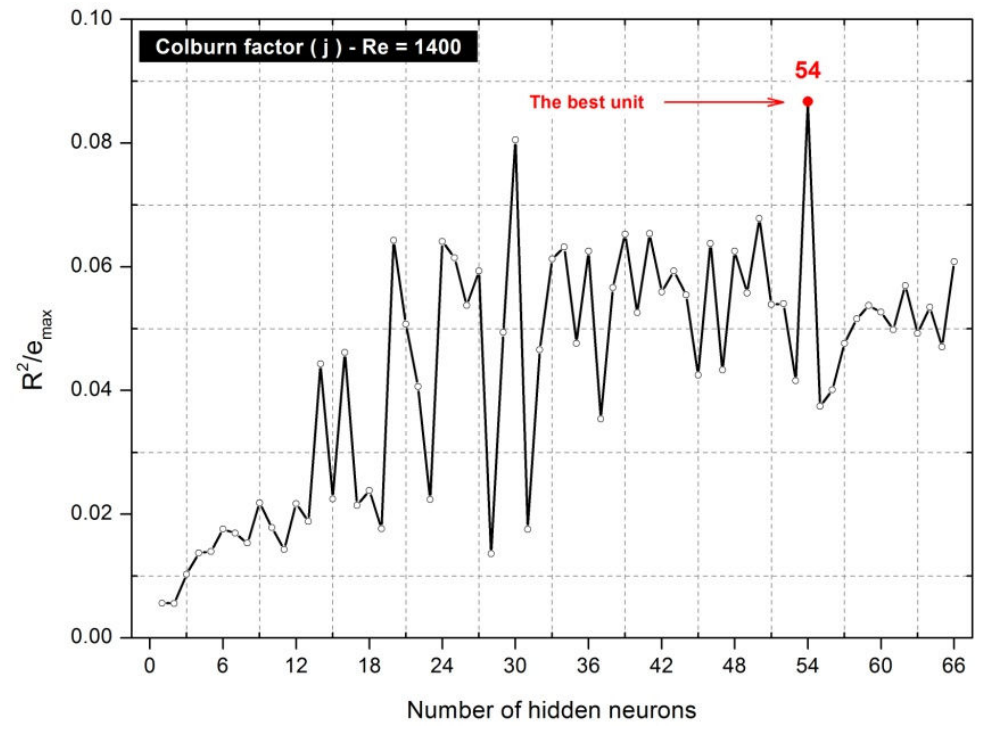

(d)

Figure $126-$ RSM training error and regression at $\mathrm{Re}=1400$ for Colburn factor (j). 
Defining the best NN is a challenge. The best practices point out that the RSM must be chosen considering the higher value for Regression $\left(R^{2}\right)$ and the smaller value for Mean Relative Error $\left(\mathrm{e}_{\text {mean }}\right)$ and Maximum Relative Error $\left(\mathrm{e}_{\max }\right)$. However, these are not always possible, as it can be seen in Figure 123 to 126. For example, in Figure $123 a$, the regression value for neuron 63 was higher than neuron 61 . Although the neuron 63 seems to be the best choice, is necessary evaluate for both $\left(e_{\text {mean }}\right)$ and $\left(e_{\max }\right)$. In this case, the neuron 61 was superior than neuron 63 for $\left(e_{\max }\right)$, Figure $123 b$, and slightly inferior than $\left(e_{\text {mean }}\right)$, Figure 123c. Again, the neuron 63 seems to be the best choice. Looking forward to the optimization step, a concern is raised: optimization solution will walk toward where? In this case, the $e_{\max }$ becomes an important parameter to successful optimization. Thus, a new parameter was analyzed $\left(R^{2} / e_{\max }\right)$, Figure $123 d$, ie, as higher this parameter is expected that the optimization procedure avoids the fake optimal solution.

This same procedure was conducted for each Friction and Colburn factors, for $\operatorname{Re}=250$ and $\operatorname{Re}=1400$. Moreover, if possible, it was chosen the smaller neuron unit. The best neuron unit chosen was indicated at each Figure 123 to 126.

Also, analyzing the results in Figure 123 to 126, the Figure $123 \mathrm{~b}$ showed that $\mathrm{e}_{\max }>10 \%(11.1 \%)$ and, in this case, higher than Forrester recommendations. However, the $e_{\text {mean }}$ was too smaller than $5 \%$ and, thus, the RSM was considered satisfactory. Figure $124 a$ also showed that the $R^{2}(0.78)$ was smaller than the Forrester recommendations. Again, analyzing the $\mathrm{e}_{\max }$ and $\mathrm{e}_{\mathrm{mean}}$, parameter were too smaller than the Forrester recommendations and, thus, was considered satisfactory. Others results meet the Forrester recommendations.

Results shown from Figure 127 to Figure 130 considered the surrogate models, briefly discussed in Chapter 4. Table 12 summarizes these RSM. Similar procedure for training and test data applied on NN, was used for all surrogate models according to Table 12. 
Table 12 - Type of meta-modeling.

\begin{tabular}{ll}
\hline $\mathrm{A} \rightarrow$ & Kriging Gaussian \\
$\mathrm{B} \rightarrow$ & Kriging Matern 5/2 \\
$\mathrm{C} \rightarrow$ & Kriging Matern 3/2 \\
$\mathrm{D} \rightarrow$ & Kriging Exponential \\
$\mathrm{E} \rightarrow$ & Kriging Rational Quadratic 0.5 \\
$\mathrm{F} \rightarrow$ & Kriging Rational Quadratic 2.0 \\
$\mathrm{G} \rightarrow$ & Neural Network \\
$\mathrm{H} \rightarrow$ & RBF Hardy's MultiQuadrics \\
$\mathrm{I} \rightarrow$ & RBF Duchon's Polyharmonic Splines \\
\hline
\end{tabular}

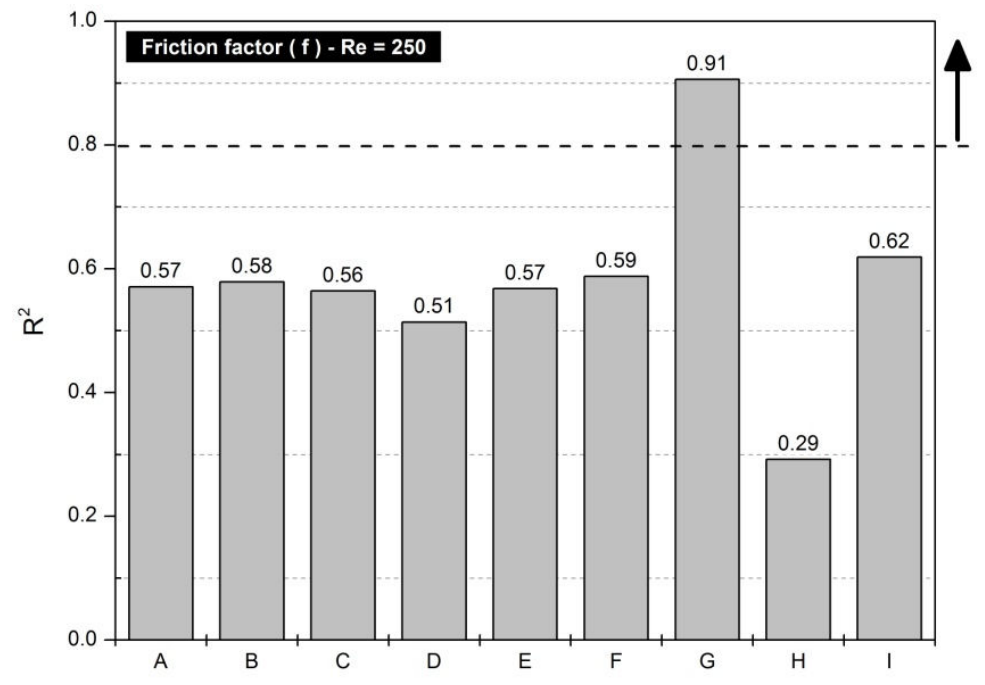

(a)

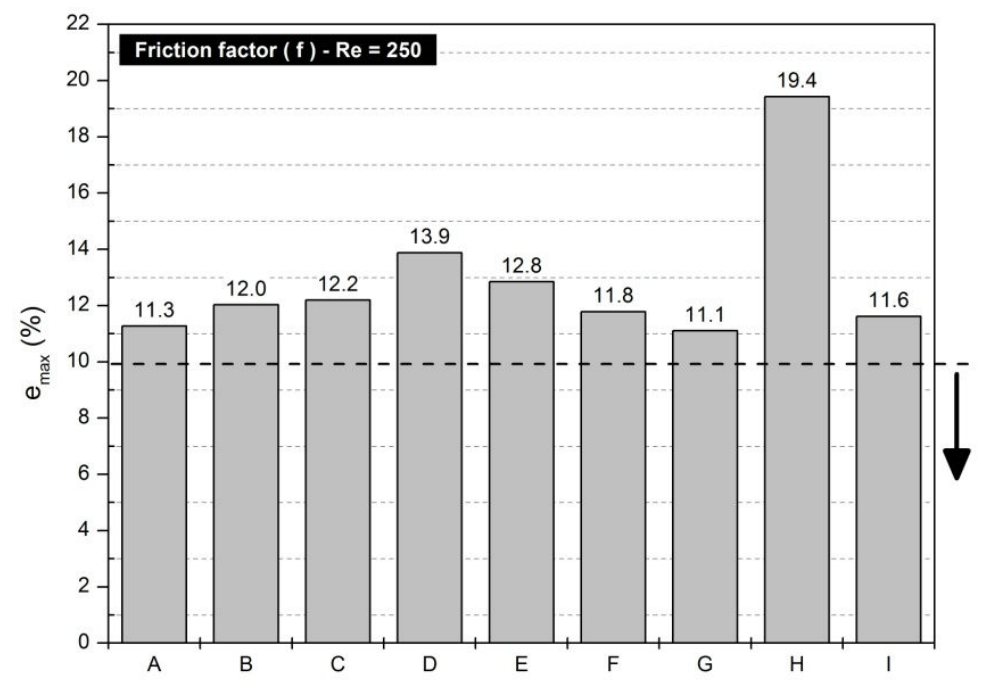

(b) 


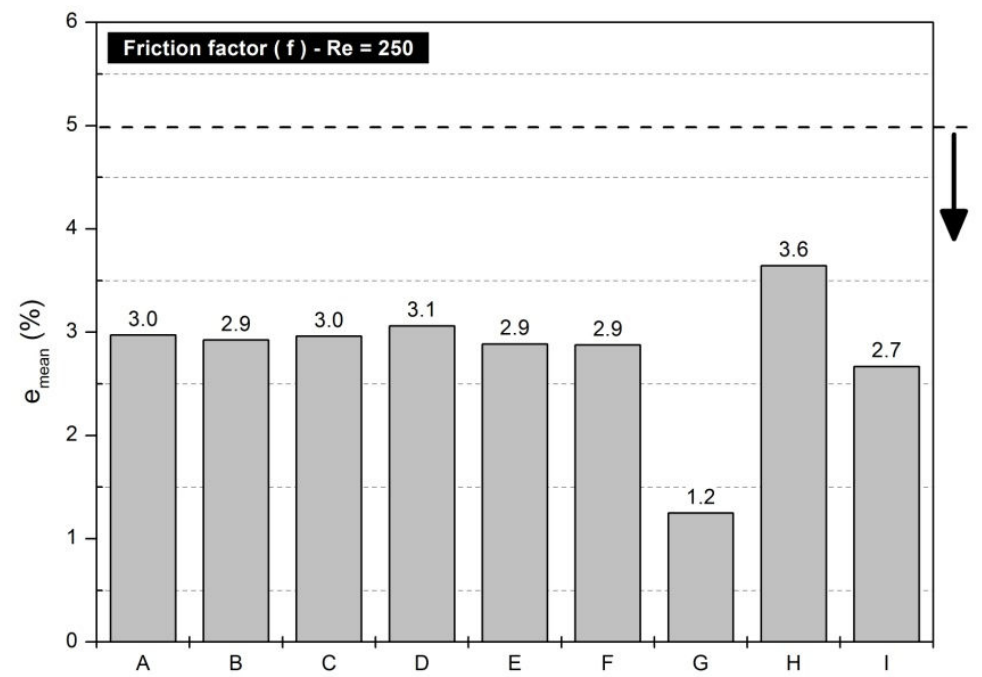

(c)

Figure 127 - Regression and error training comparison for different meta-modeling method at $R e=250$ for Friction factor (f) according to Table 12.

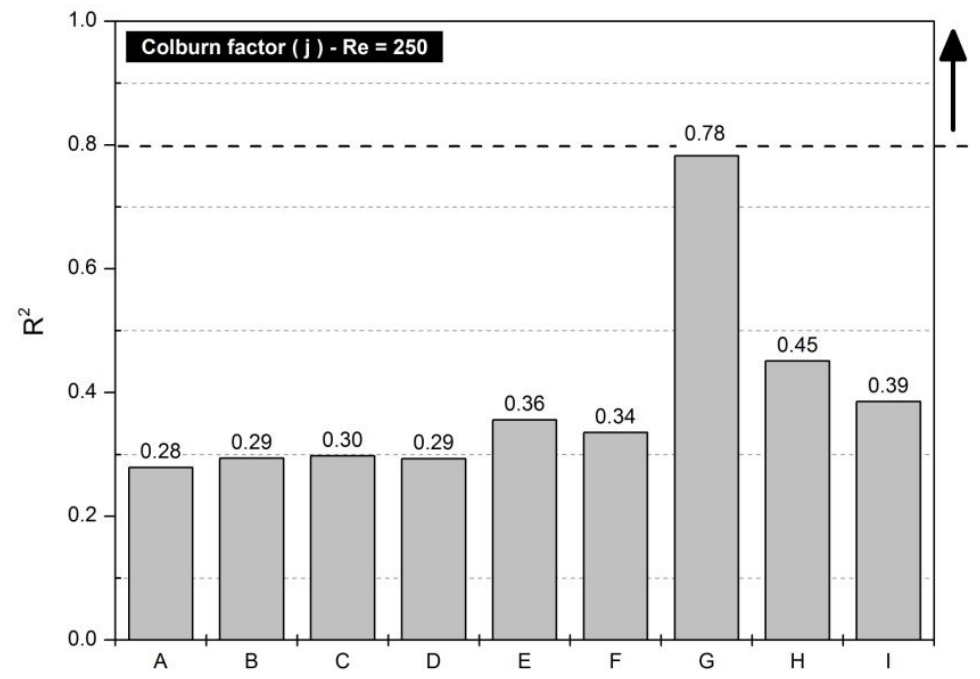

(a)

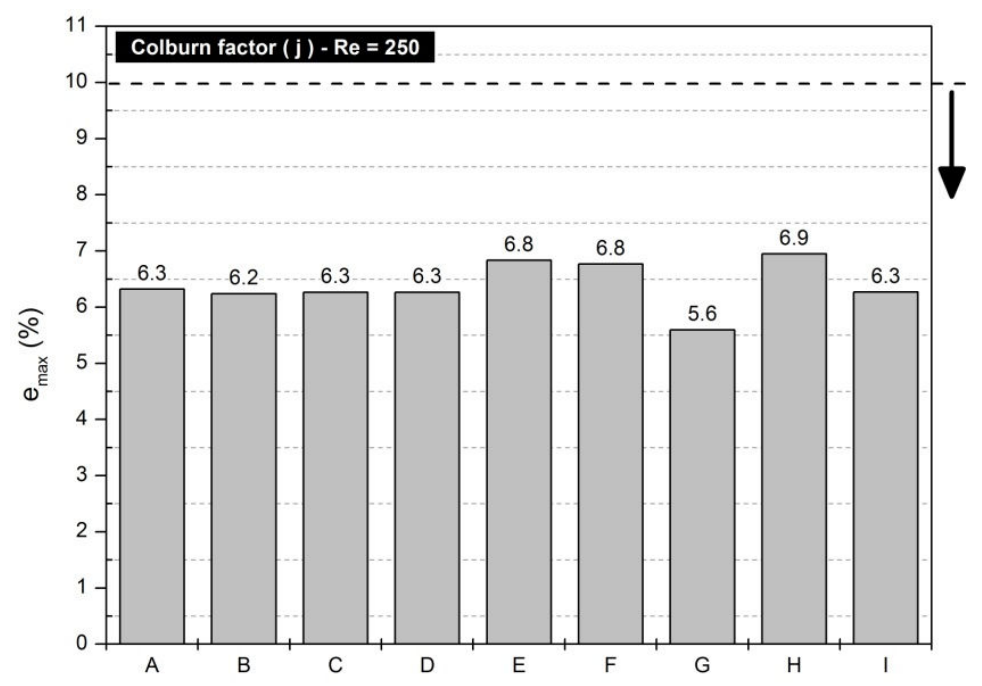

(b) 


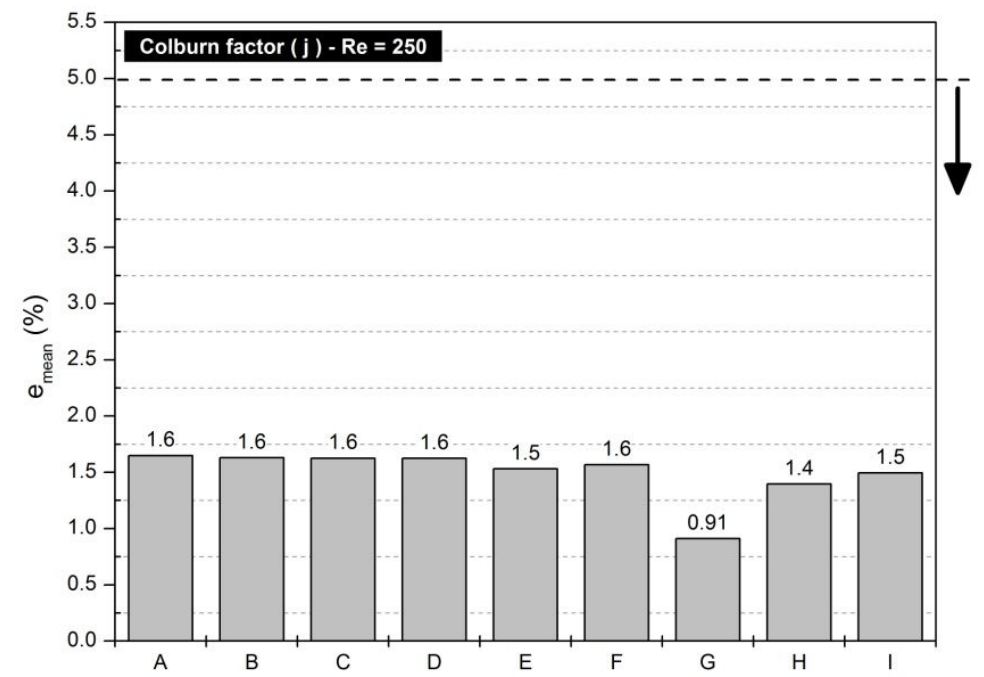

(c)

Figure 128 - Regression and error training comparison for different meta-modeling method at $\operatorname{Re}=250$ for Colburn factor (j) according to Table 12.

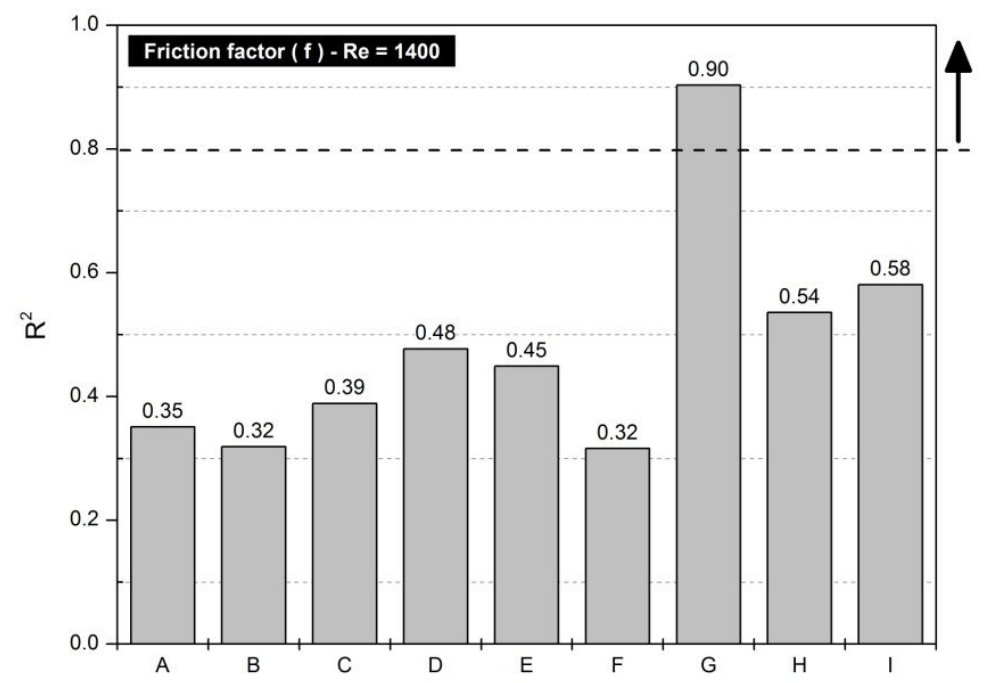

(a)

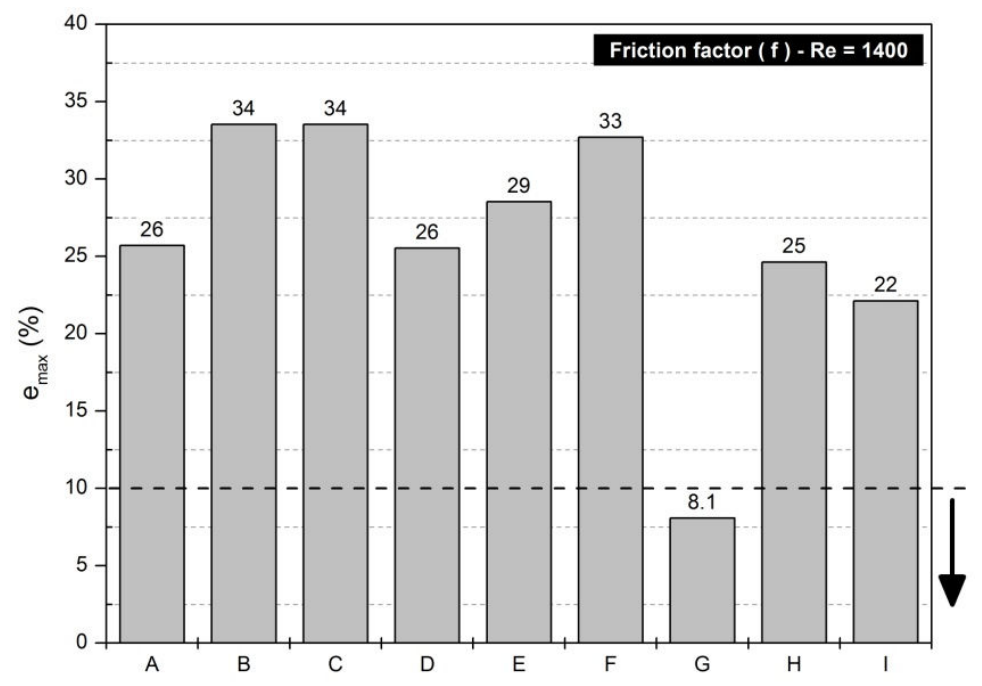

(b) 


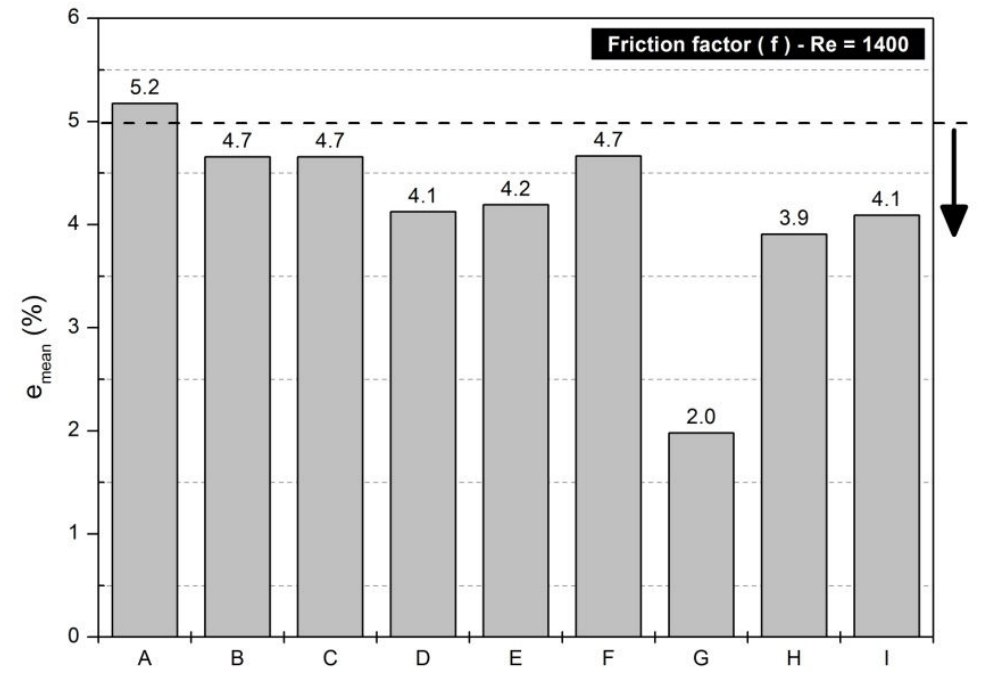

(c)

Figure 129 - Regression and error training comparison for different meta-modeling method at $\mathrm{Re}=1400$ for Friction factor (f) according to Table 12.

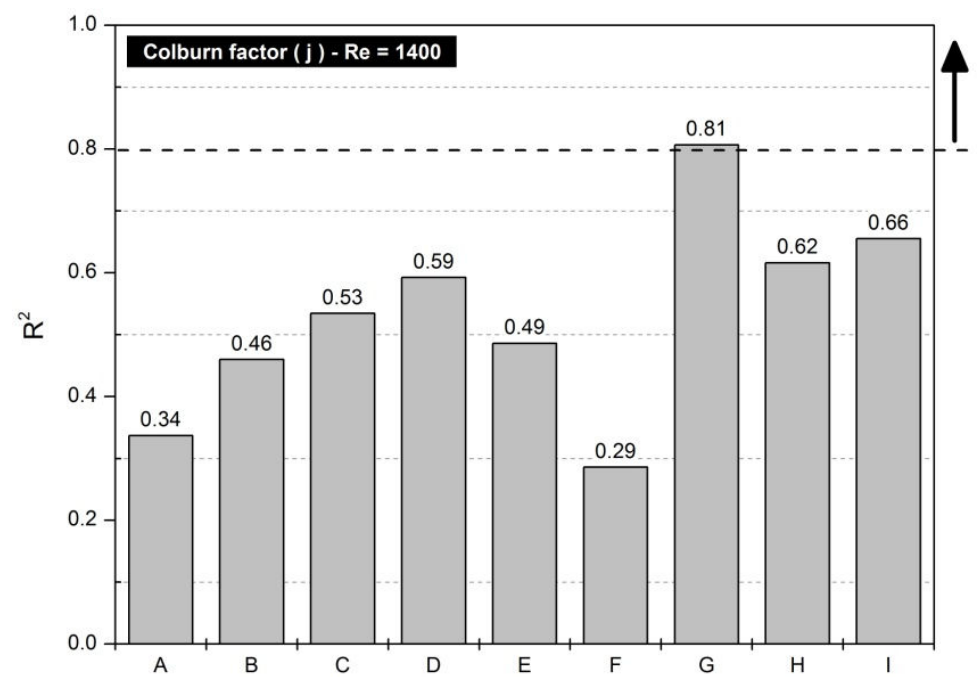

(a)

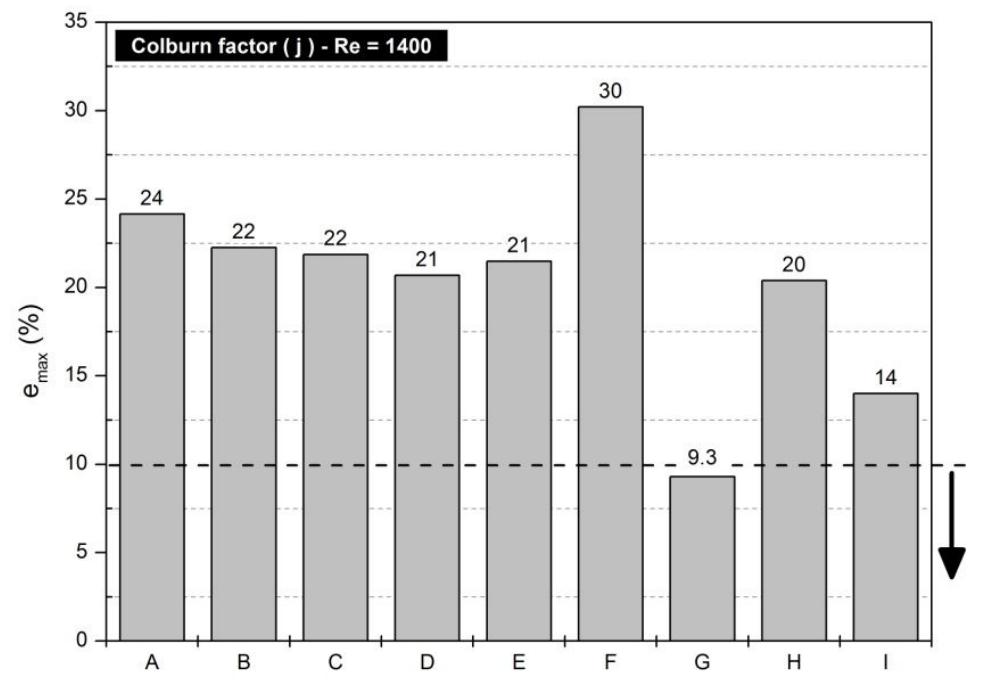

(b) 


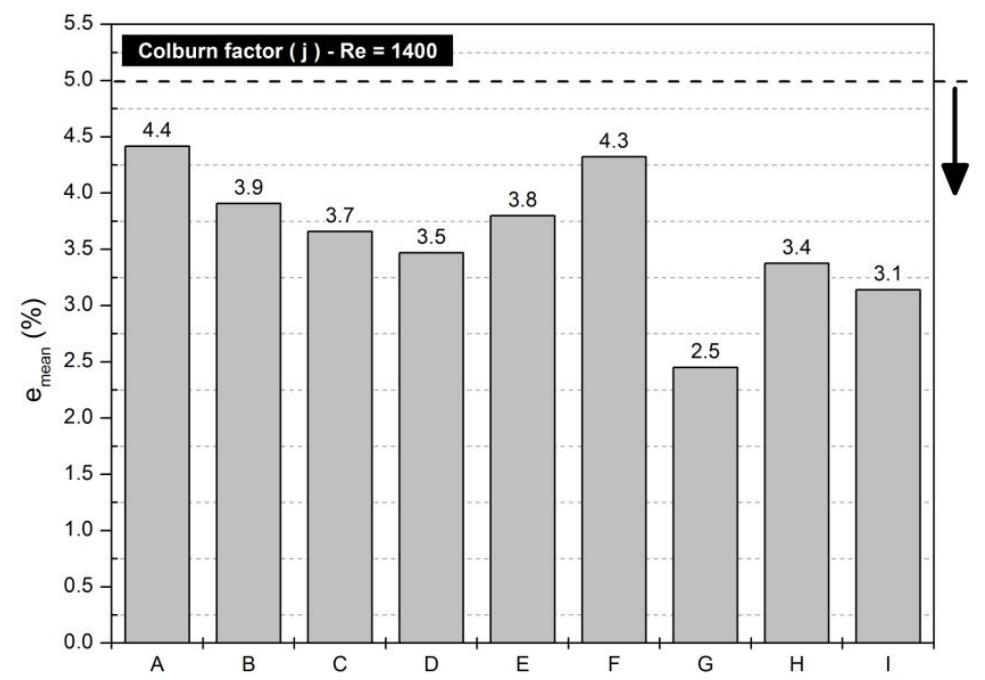

(c)

Figure 130 - Regression and error training comparison for different meta-modeling method at $\mathrm{Re}=1400$ for Colburn factor (f) according to Table 12 .

Figure 127 to Figure 130 showed notable superiority of the Neural Network on the others meta-modeling methods, especially evaluating the Regression results, Figure $127 a$ to Figure 130a. Mean Relative Error $\left(e_{\text {mean }}\right)$ meets the Forrester recommendations for all RSM's, except to Friction factor (f) at $\operatorname{Re}=1400$, Figure 129c $(5.2 \%)$. However, this result could be considered satisfactory. The Maximum Relative Error $\left(e_{\max }\right)$ showed the major differences. For Friction and Colburn factors and $\operatorname{Re}=1400$, Figure $129 \mathrm{~b}$ and Figure $130 \mathrm{~b}$, except to $\mathrm{NN}$, the $\mathrm{e}_{\max }$ reached $34 \%$ and $30 \%$, respectively. The maximum error is a great concern considering the optimization step. Therefore, NN showed stronger capability of generalization than others meta-modeling methods, consolidating the initial choice for Neural Network. Because the long-time for optimization procedure and the last assessments, only NN was applied to find optimal designs and the optimal solution for different RSM could be a matter for future works. 